U.S. Department of the Interior

U.S. Geological Survey

\title{
Results of Soil, Ground-Water, Surface-Water, and Streambed-Sediment Sampling at Air Force Plant 85, Columbus, Ohio, 1996
}

By James M. Parnell

Open-File Report 97-641

Prepared in cooperation with the

Department of the U.S. Air Force

Aeronautical Systems Center

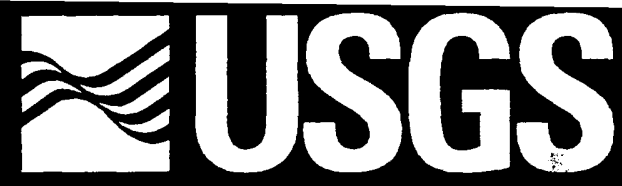




\title{
U.S. DEPARTMENT OF THE INTERIOR \\ BRUCE BABBITT, Secretary
}

\author{
U.S. GEOLOGICAL SURVEY \\ Mark Schaefer, Acting Director
}

Any use of trade, product, or firm names is for descriptive purposes only and does not constitute endorsement by the U.S. Government.

For additional information write to:

Copies of this report can be purchased from:

District Chief

U.S. Geological Survey

975 West Third Avenue

Columbus, Ohio 43212-3192
U.S. Geological Survey

Information Services

Box 25286

Denver Federal Center

Denver, CO 80225-0286 


\section{CONTENTS}

Abstract

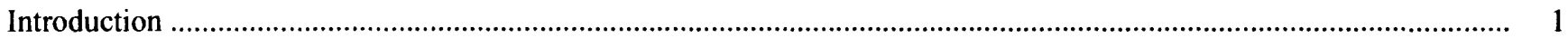

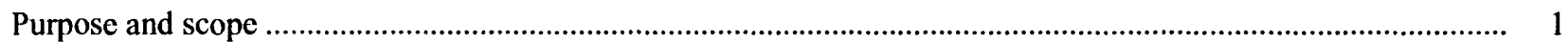

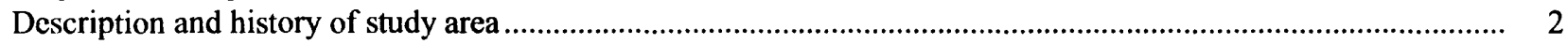

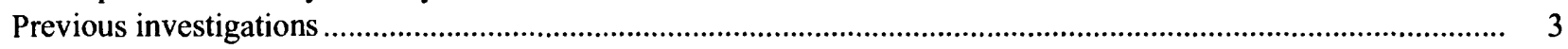

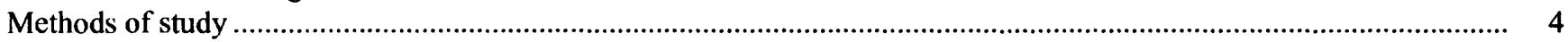

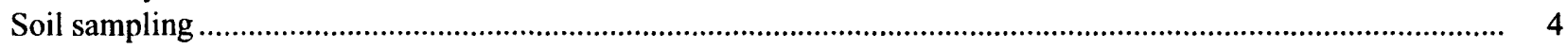

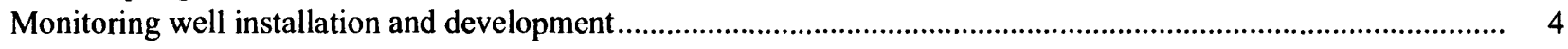

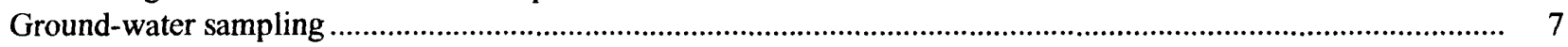

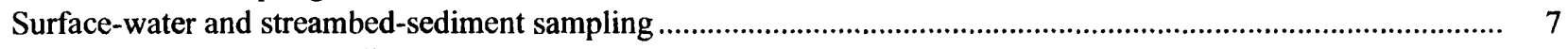

Quality-assurance and quality-control measures ............................................................................................ 8

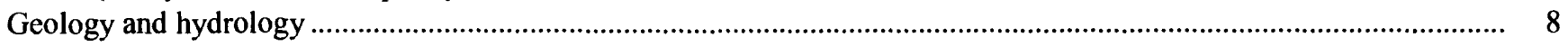

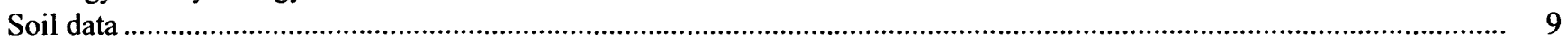

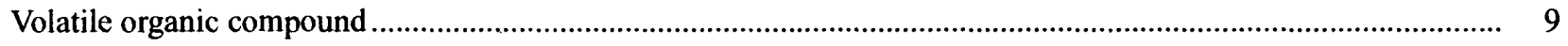

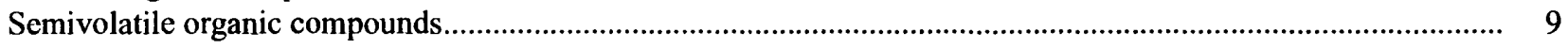

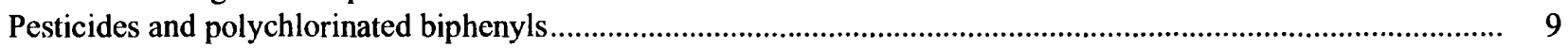

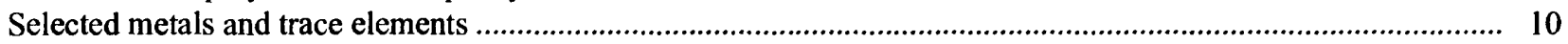

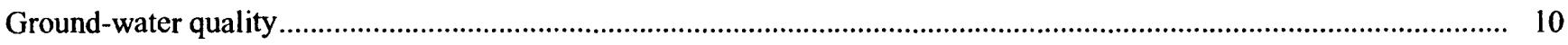

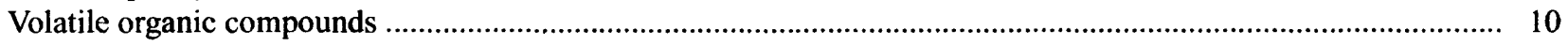

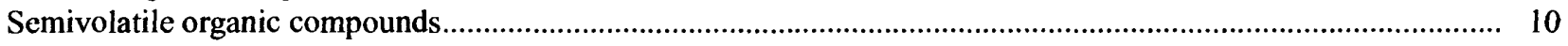

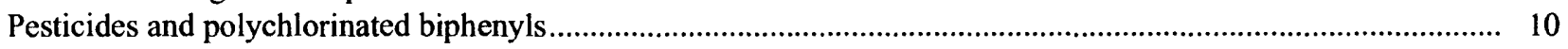

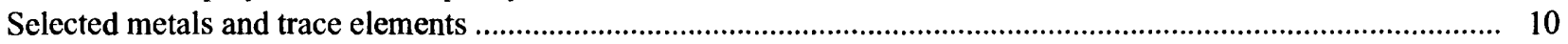

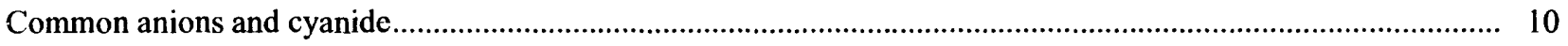

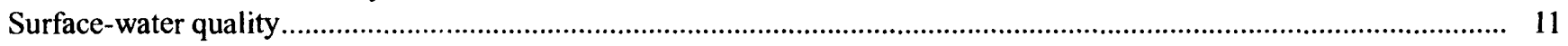

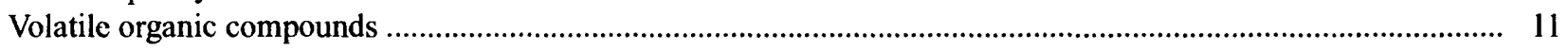

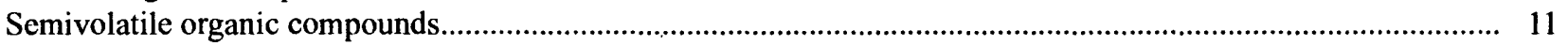

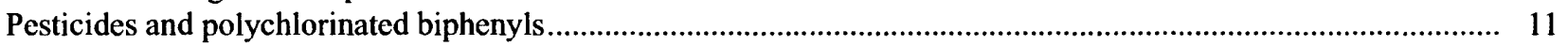

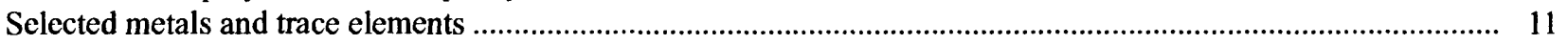

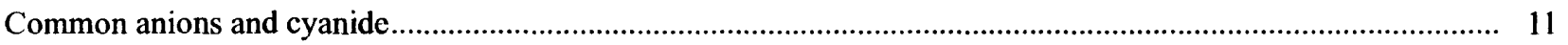

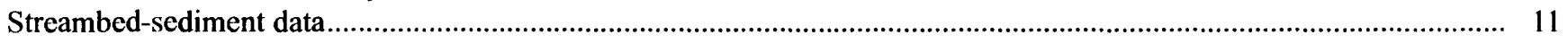

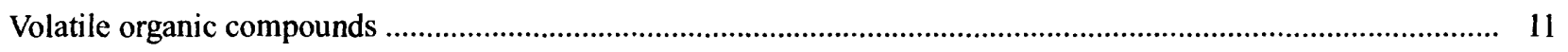

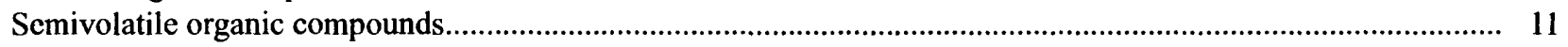

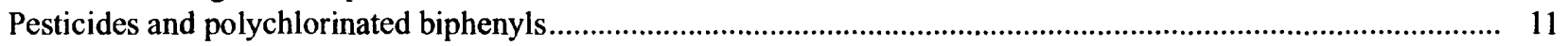

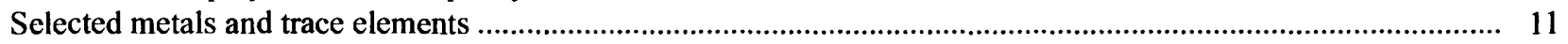

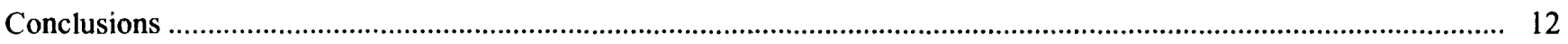

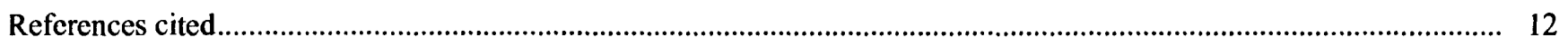

Appendixes:

1. Logs of borings at Air Force Plant 85, Columbus, Ohio, 1996 ....................................................................... 14

2. Construction diagrams for observation wells at Air Force Plant 85, Columbus, Ohio, 1996 ............................ 38

3. Summary data: analytical results for soil, ground water, surface water, and streambed sediment at Air Force Plant 85, Columbus, Ohio, 1996.

\section{FIGURES}

1-11. Maps showing:

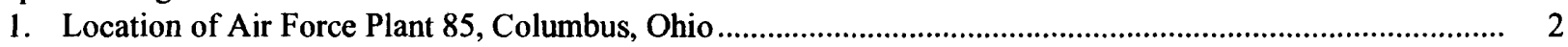

2. Location of sampling areas and existing study sites,Air Force Plant 85 , Columbus, Ohio ............................ 3 
3. Soil boring/sampling locations at magnesium-chip burning and rubble-disposal area, Air Force Plant 85 , Columbus, Ohio

4. Soil boring/sampling locations near the coal pile, Air Force Plant 85 , Columbus, Ohio

5. Soil boring/sampling locations north of Building 3 and at fuel tank area east of Building 3 , Air Force Plant 85 , Columbus, Ohio

6. Soil boring/sampling locations on the north side of Air Force Plant 85 (north-central boundary, wash rack area, and near Mason's Run), Columbus, Ohio.

7. Soil boring/sampling locations at leach field area, stationary radar site, and northwest perimeter road, Air Force Plant 85, Columbus, Ohio

8. Soil boring/sampling locations on the southwestern part of Air Force Plant 85 (south of Buildings 124 and 125 and in southwest Building 4 parking lot), Columbus, Ohio

9. Soil boring/sampling location southwest of Building 3 (in parking lot), Air Force Plant 85,

Columbus, Ohio

10. Surface-water and streambed-sediment sampling locations on Mason's Run, Air Force Plant 85,

Columbus, Ohio

11. Surface-water and streambed-sediment sampling locations on Turkey Run, Air Force Plant 85,

Columbus, Ohio.

12. Generalized cross section showing geology of Air Force Plant 85, Columbus, Ohio

13. Cross section A-A' showing monitoring wells at Air Force Plant 85, Columbus, Ohio

\section{TABLES}

1. Location, area, and site identification of boring, ground-water, and surface-water sites,

Air Force Plant 85, Columbus, Ohio

2. Analyte group, analytical method, and sample matrix

\section{CONVERSION FACTORS, VERTICAL DATUM, AND ABBREVIATED WATER-QUALITY UNITS}

$\begin{array}{rcl}\text { Multiply } & \text { By } & \text { To obtaln } \\ \text { inch } & 25.4 & \text { millimeter } \\ \text { foot } & 0.3048 & \text { meter } \\ \text { acre } & 0.4047 & \text { square hectometer } \\ \text { gallon } & 3.785 & \text { liter } \\ \text { pound, avoirdupois } & 0.4536 & \text { kjlogram }\end{array}$

Temperature is given in degrees Celsius $\left({ }^{\circ} \mathrm{C}\right)$, which can be converted to degrees Fahrenheit $\left({ }^{\circ} \mathrm{F}\right)$ by use of the following equation:

$$
{ }^{\circ} \mathrm{F}=1.8\left({ }^{\circ} \mathrm{C}\right)+32
$$

Sea level: In this report, "sea level" refers to the National Geodetic Vertical Datum of 1929 (NGVD of 1929) - a geodetic datum derived from a general adjustment of the first-order level nets of both the United States and Canada, formerly called Sea Level Datum of 1929.

Abbreviated chemical and physical units used in this report: Chemical concentrations and water temperature are given in metric units. Chemical concentration is given in milligrams per liter $(\mathrm{mg} / \mathrm{L})$ or micrograms per liter $(\mu \mathrm{g} / \mathrm{L})$. Milligrams per liter is a unit expressing the concentration of chemical constituents in solution as weight (nilligrams) of solute per unit volume (liter) of water. One thousand micrograms per liter is equivalent to one milligram per liter. For concentrations less than $7,000 \mathrm{mg} / \mathrm{L}$, the numerical value is approximately the same as for concentrations in parts per million. Specific conductance of water is expressed in microsiemens per centimeter at 25 degrees Celsius $(\mu \mathrm{S} / \mathrm{cm})$. This unit is equivalent to micromhos per centimeter at 25 degrees Celsius $(\mu \mathrm{mho} / \mathrm{cm})$, formerly used by the U.S. Geological Survey.

Chemical concentrations of constituents in a solid matrix are given in milligrams per kilogram ( $\mathrm{mg} / \mathrm{kg})$. These units are used to express the concentrations of chemical constituents as weight of consituent concentrations in soils. Volumes of headspace gas screenings are given in microliters $(\mu \mathrm{L})$. 


\section{ABBREVIATIONS AND ACRONYMS}

AFP 85

DOD

I.D.

IRP

O.D.

PCB's

Plant

PVC.

QA/QC

SVOC's

USEPA

USGS

VOC's
Air Force Plant 85

Department of Defense

Inside diameter

Installation Restoration Program

Outside Diameter

Polychlorinated biphenyls

Air Force Plant 85

Polyvinyl chloride

Quality assurance and quality control

Semivolatile organic compounds

U.S. Environmental Protection Agency

U.S. Geological Survey

Volatile organic compounds 


\title{
Results of Soil, Ground-Water, Surface-Water, and Streambed-Sediment Sampling at Air Force Plant 85, Columbus, Ohio, 1996
}

\author{
By James M. Parnell
}

\section{ABSTRACT}

The U.S. Geological Survey (USGS), in cooperation with Aeronautical Systems Center, Environmental Management Directorate, Restoration Division, prepared the Surface-Water and Ground-Water Monitoring Plan for Air Force Plant 85 (AFP 85 or Plant), Columbus, Ohio, under the Air Force Installation Restoration Program to characterize any ground-water, surfacewater, and soil contamination that may exist at AFP 85. The USGS began the study in November 1996.

The Plant was divided into nine sampling areas, which included some previously investigated study sites. The investigation activities included the collection and presentation of data taken during drilling and water-quality sampling. Data collection focused on the saturated and unsaturated zones and surface water. Twenty-three soil borings were completed. Ten monitoring wells (six existing wells and four newly constructed monitoring wells) were selected for water-quality sampling. Surface-water and streambed-sediment sampling locations were chosen to monitor flow onto and off of the Plant. Seven sites were sampled for both surface-water and streambed-sediment quality.

This report presents data on the selected inorganic and organic constituents in soil, ground water, surface water, and streambed sediments at AFP 85. The methods of data collection and analysis also are included. Knowledge of the geologic and hydrologic setting could aid Aeronautical Systems Center, Environmental Management Direc- torate, Restoration Division, and its governing regulatory agencies in future remediation studies.

\section{INTRODUCTION}

In 1980, the Department of Defense (DOD) devised a comprehensive Installation Restoration Program (IRP) to assess and to control the migration of environmental contamination that may have resulted from past operations and (or) disposal practices at DOD facilities. The IRP underwent changes under the Superfund Amendment/Reauthorization Act in 1986, which required all DOD facilities to follow guidelines and procedures set forth by the U.S. Environmental Protection Agency (USEPA). The U.S. Geological Survey (USGS), in cooperation with Aeronautical Systems Center, Environmental Management Directorate, Restoration Division, prepared the Surface-Water and Ground-Water Monitoring Plan (Parnell, 1996) for Air Force Plant 85 (AFP 85 or Plant), Columbus, Ohio, under the Air Force IRP to characterize any groundwater, surface-water, and soil contamination that may exist at AFP 85. The USGS began the study in November 1996.

\section{Purpose and Scope}

This report presents data on selected inorganic and organic constituents in soil, ground water, surface water, and streambed sediments at AFP 85. The methods of data collection and analysis also are included. Knowledge of the hydrogeologic system could aid Aeronautical Systems Center, Environmental Management Directorate, Restoration Division, and its governing regulatory agencies during future remediation sutides in determining the potential pathways for 
contaminant migration into the underlying water-bearing zones or nearby surface waters

\section{Description and History of Study Area}

AFP 85 is in Franklin County in the eastern part of Columbus, Ohio. The 288-acre site is in an urban industrial area adjacent to Port Columbus International Airport (fig. 1). AFP 85 began operations in 1941 as a naval aircraft production and maintenance facility. In 1982, the Plant was transferred to the U.S. Air Force from the U.S. Navy. Shortly thereafter, Rockwell International was awarded the contract to produce the B-1 bomber at AFP 85. Starting in 1988, the Plant was operated for the U.S. Air Force by McDonnell-Douglas for the production of aircraft parts. In 1994, McDonnell-Douglas ceased operations. Since that time, the facility has been used for administration purposes only by the Defense Finance Accounting Service.

Over the years at AFP 85, operations included machining, metal finishing and electroplating, forming, painting and coating, and assembly. Waste associated with these activities included concentrated acids for metal cleaning and electroplating, process-tank sludges, cyanide wastes, chromium solutions, lime sludges, spent degreasers, and paint strippers. Waste oils, solvents, and aviation fuels were also collected and stored as part of the operations.

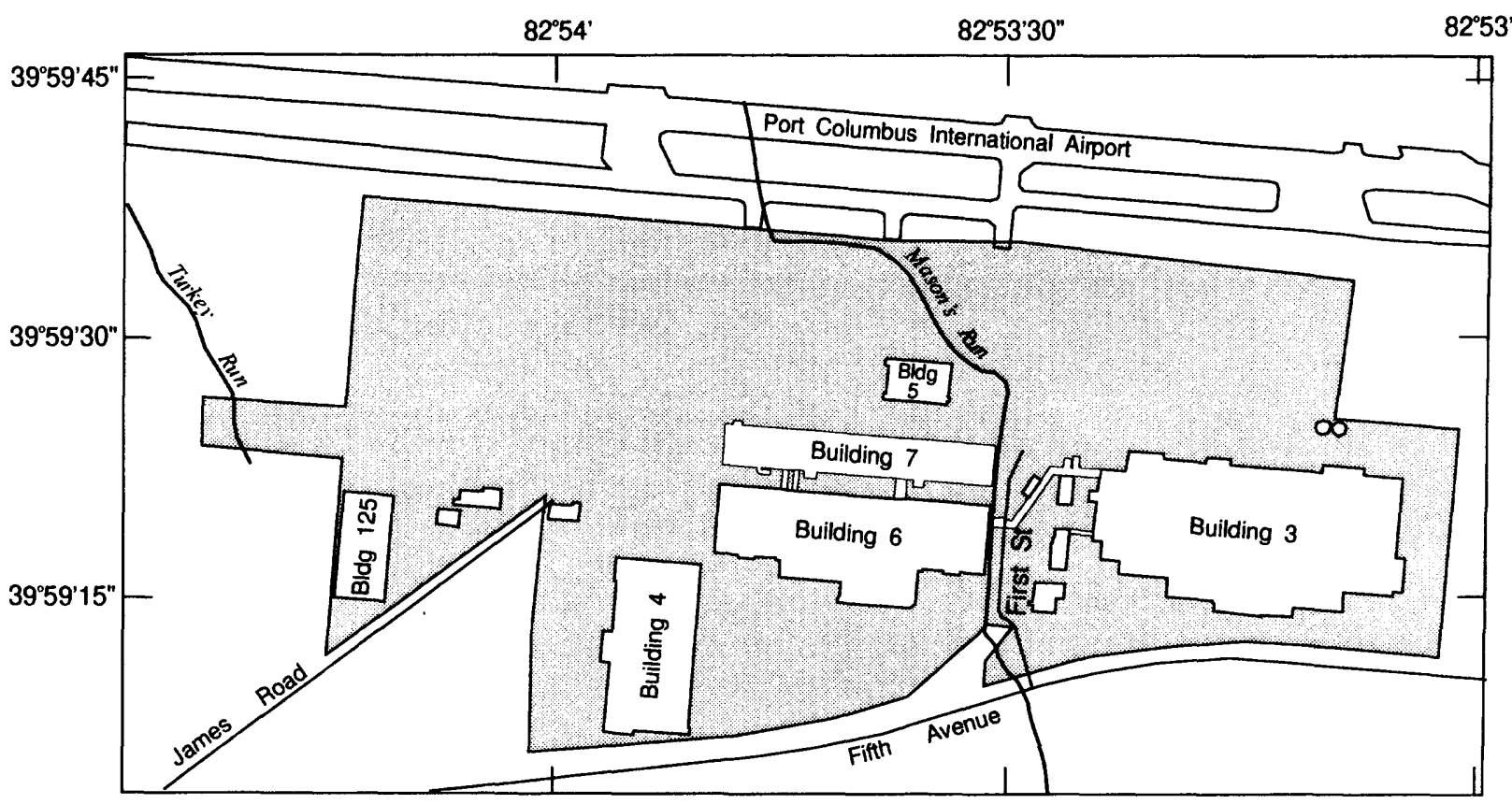

Base modified from U.S. Naval Weapons Industrial Reserve Plant, Columbus, Ohio, 1976

EXPLANATION

AIR FORCE PLANT 85 PROPERTY
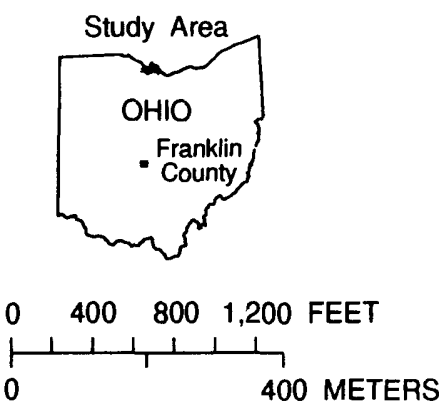

Figure 1. Location of Air Force Plant 85, Columbus, Ohio. 


\section{Previous Investigations}

AFP 85 has been the subject of a several investigations since 1982. The following briefs are based on a review of reports and supporting documents submitted by consultants and from project files (U.S. Air Force Aeronautical Systems Center, 1994); existing sites are shown in figure 2.

The IRP began in 1982 under a Phase I Hazardous Materials Disposal Site Records Search. The purpose of this study was to identify sites of potential environmental concern. Nine sites $(1,2,3,4,5,6,7,9$, and 11) at AFP 85 were identified in the Phase I report.

A Phase II, Stage I, Investigation Confirmation and Quantification was completed in 1988. During the investigation, limited sampling was done at Sites 2 through 5 and 8 .
A Phase II, Stage II, Remedial Investigation and Feasibility Study was completed in 1990. The work was completed in accordance with the National Contingency Plan enacted under the Comprehensive Environmental Response, Compensation, and Liability Act. This work involved Sites 3, 4, 5, 8, and 10 and resulted in No Further Action Decision Documents.

In 1993, a Phase II Stage 2A study was done at Sites 3 and 4. Additional work was done at Site 11 under the Toxic Substances Control Act.

Site characterization work at Site 4 by the USGS and U.S. Army Corps of Engineers was done in September 1994. A U.S. Army Corps of Engineers data report was used to define the extent of possible contamination by organic compounds at Site 4 .

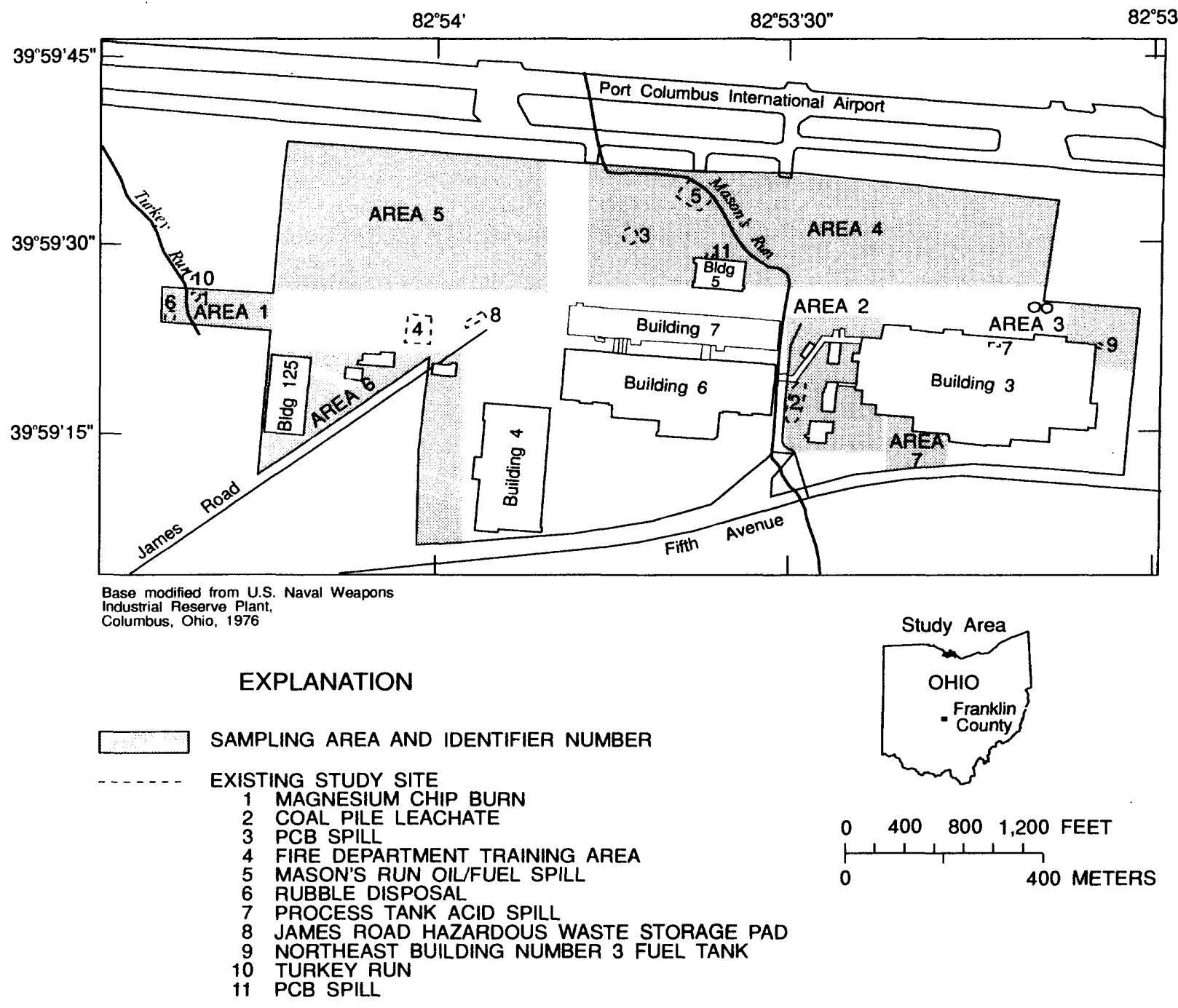

Figure 2. Location of sampling areas and existing study sites, Air Force Plant 85, Columbus, Ohio. 


\section{METHODS OF STUDY}

This report presents the data collected during drilling and water-quality sampling. Data collection focused on the saturated and unsaturated zones and surface water. The various Plant areas are listed in table 1 and are shown in detail in figures 3 through 11 (at back of report).

\section{Soil Sampling}

A total of 23 soil borings were completed to determine the levels of anthropogenic and nonanthropogenic chemical compounds in the vicinity of the selected Plant areas. The size and specific location of each area was determined from previous investigations and (or) from aerial photographs if they were available. The total number of borings for each area were selected to be vertically and areally representative of the area. Two samples were selected from each boring on the basis of field-screening criteria described below and were sent to the contract laboratory for analysis. Total depths range from 2.3 to 95 feet.

Borehole drilling was done by use of an 8-1/2-inch-inside-diameter (I.D.) hollow-stem auger. The continuous soil samples were collected for analysis by use of a 60 -inch-long, 4-inch-outer-diameter (O.D.) core-barrel sampler. The sampler was driven 30 inches or to refusal. Once driven, the barrel and rod were withdrawn from the auger and the sampler was removed from the rods. USGS personnel recorded descriptive (lithologic) logs in the field notebook (Appendix 1). Soil samples for chemical analysis consisted of qualitative field screening followed by quantitative laboratory analyses. For the field screening, a portable organic vapor analyzer equipped with a flame ionization detector and a photoionization detector was used to analyze the borehole samples for volatile organic compounds (VOC's), progressing from one end of the core to the other. Sections of core identified as potentially contaminated by the analysis were preferentially selected for the subsequent laboratory analyses. Other samples were collected and sent to the laboratory on the basis of lithology. All borehole cuttings were drummed for later analysis and disposal by the Plant. All boreholes were grouted by injecting a bentonite/cement slurry until it overflowed at the surface. Analyses for soil samples are listed in table 2. Analytical procedures used are outlined in the Surface- water and Ground-water Monitoring Plan (Parnell, 1996). The results of the borehole screening are included on the boring logs in Appendix 1.

\section{Monitoring Well Installation and Development}

When a sufficient volume of ground water available to run selective chemical analysis was found during soil-sampling drilling, a new monitoring well was installed within the significantly permeable zone. A total of four new monitoring wells were drilled for this study. Monitoring wells were constructed of 2-inchI.D. polyvinyl chloride (PVC) Schedule 40 well casing and screens. The screens were flush threaded with a bottom plug. Slot size was 0.010 inch. Both 5 -foot and 10-foot screen lengths were used, as determined by the borehole lithology. The screen was packed with a clean silica sand to 3 feet above the top of the screen. The final depth of the filter pack was sounded with a weighted metal tape. At least 2.5 feet of bentonite pellets was placed on top of the filter pack. The annulus was grouted with a cement/bentonite grout to the frost line. The grout mix consisted of one 94-pound sack of Portland cement, 3 pounds of bentonite, and 6.5 gallons of potable water. Above-ground and below-ground monitoring (flush-mount) well completions were used. A 5-foot protective casing with a hinged lockable lid was used for above-ground completions, whereas a 10 -inch flush-mount casing with a locking top was used for below-ground completions. A sloping concrete pad was constructed around each well to prevent surface-water intrusion. As-built well-construction diagrams showing the placement of the well screen, sand pack, bentonite seal, and grouting are compiled in Appendix 2. Construction details of existing wells are not presented in this report; for additional information, see U.S. Air Force Aeronautical Systems Center (1994).

All monitoring wells were developed by use of a precleaned disposable bailer and (or) a low-flow submersible pump. A minimum of 24 hours was allowed between well installation and well development. When the well was purged dry, the well was sampled upon recovery and within 24 hours. Specific conductance, $\mathrm{pH}$, temperature, and turbidity were monitored during the development. Development continued until three consecutive readings of $\mathrm{pH}$, specific conductance, 


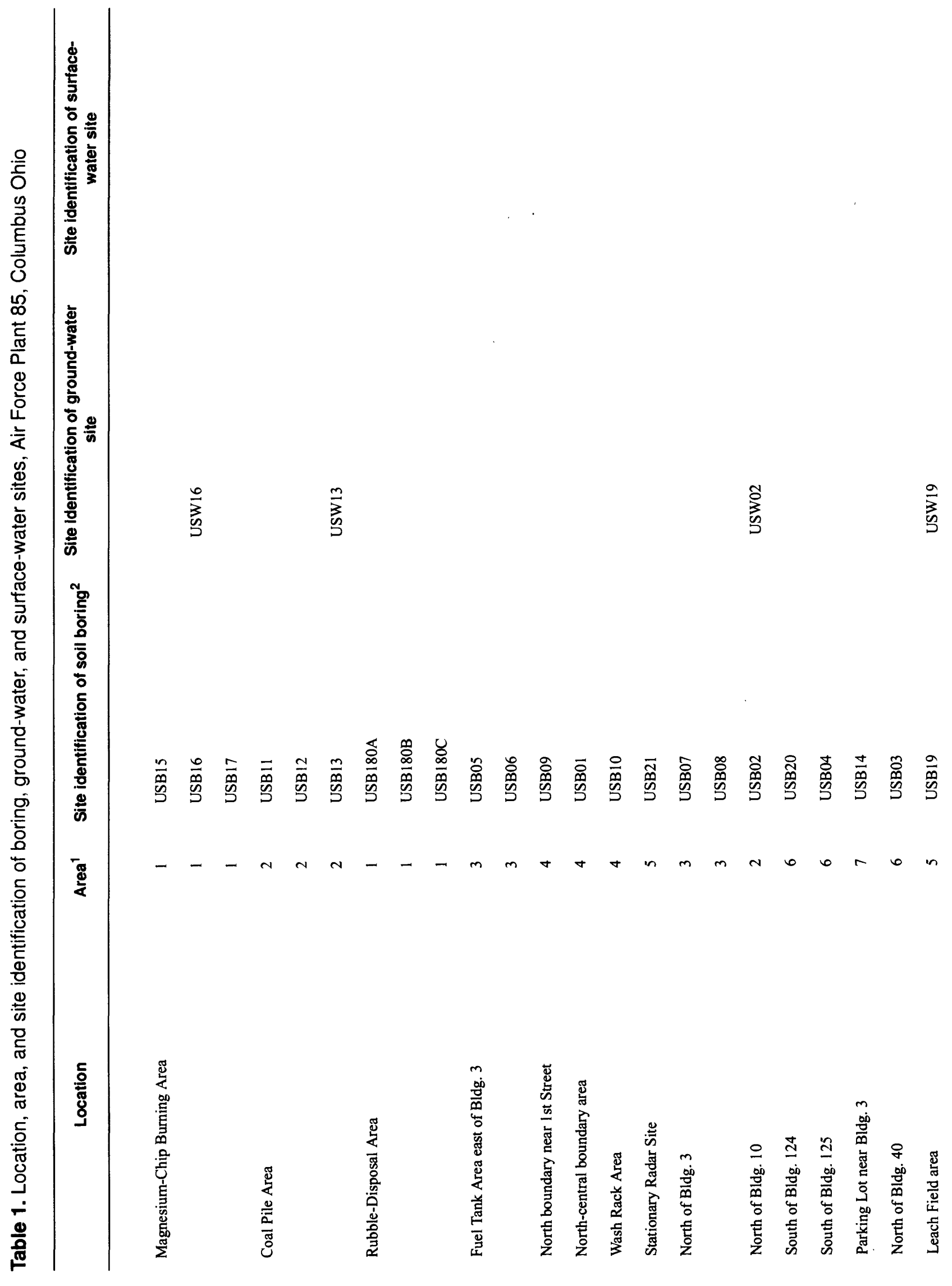




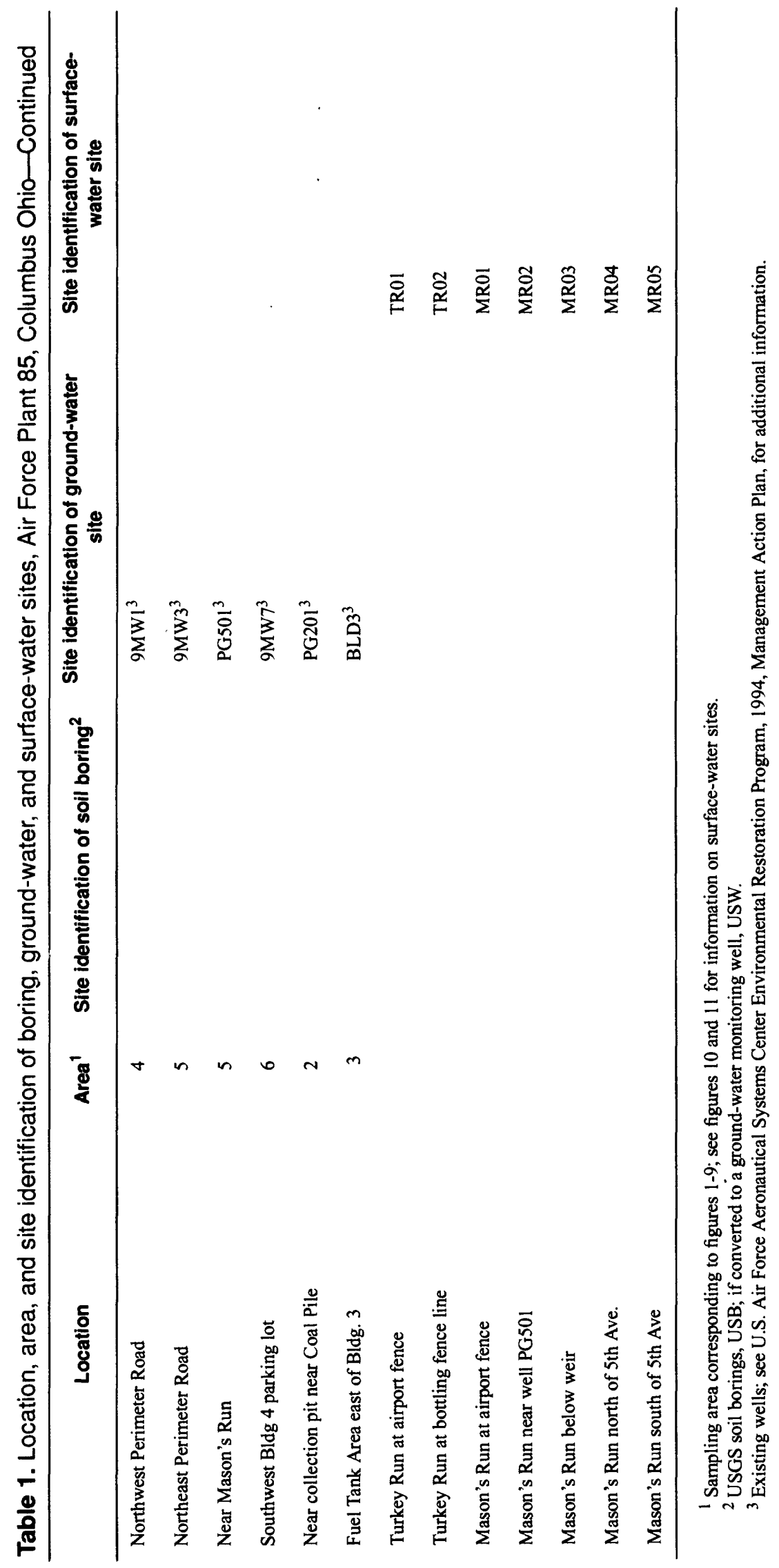


temperature, and turbidity stabilized. Field-measured characteristics were considered stable when $\mathrm{pH}$ 's were within \pm 0.1 unit, specific conductances were within \pm $10 \mu \mathrm{S} / \mathrm{cm}$, and temperatures were within $\pm 0.5^{\circ} \mathrm{C}$. Development time, volume of water produced, and field measurements were recorded in the field logbook. All water produced during development, and later during decontamination activities, was contained at the field site. All equipment was thoroughly cleaned and decontaminated between locations to prevent crosscontamination, as outlined in the Surface-Water and Ground-Water Monitoring Plan (Parnell, 1996).

\section{Ground-Water Sampling}

Ground-water sampling locations were selected to determine the levels of anthropogenic and nonanthropogenic chemical compounds in the vicinity of the selected Plant areas. A total of 10 monitoring wells were selected for this study. Six existing wells were inventoried and selected for sampling, and the four newly constructed monitoring wells also were selected.

Prior to purging and sampling, depth to water was measured to the nearest 0.01 foot. The volume of standing water in the well and in the sand pack below the grout seal were then calculated as one purge volume. Three purge volumes were removed in each well to replace the stagnant water with fresh water from the water-bearing formation. All monitoring wells were purged by use of a precleaned disposable bailer and (or) a low-flow submersible pump. If the well was purged dry, the well was sampled upon recovery and within 24 hours. A minimum of 10 well volumes of water, if possible, was removed from each well before sampling where turbidity was persistent (Ohio Environmental Protection Agency, 1995). The water samples were collected by filling the pre-preserved sample container either directly from the precleaned or dedicated bailer and (or) from a low-flow-rate discharge tube from the submersible pump. After collection, the samples were chilled and shipped by overnight courier to the contract laboratory (Quanterra Environmental Services, Denver, Colo.) for analysis. Analyses for ground-water samples are listed in table 2. Analytical procedures and standard operating procedures are outlined in the Surface-Water and Ground-Water Monitoring Plan (Parnell, 1996).
Table 2. Analyte group, analytical method, and sample matrix.

\begin{tabular}{|c|c|c|}
\hline $\begin{array}{l}\text { Analyte } \\
\text { group } 1\end{array}$ & $\begin{array}{l}\text { Analytical } \\
\text { method }^{2}\end{array}$ & Sample matrix ${ }^{3}$ \\
\hline $\begin{array}{l}\text { Volatile organic compounds } \\
\text { by GC/MS }\end{array}$ & SW8260/8240 & S-GW-SW-BM \\
\hline $\begin{array}{l}\text { Semivolatile organic com- } \\
\text { pounds by GC/MS }\end{array}$ & SW8270 & S-GW-SW-BM \\
\hline Pesticides and PCB's & SW8080 & S-GW-SW-BM \\
\hline Metals & SW6010 & S-GW-SW-BM \\
\hline Common anions & E300.0 & GW-SW \\
\hline Cyanide & SW9012 & SW-GW \\
\hline
\end{tabular}

' GC/MS, gas chromatography/mass spectrometry; PCB's, polychlorinated biphenyls:

${ }^{2}$ Analytical methods SW and E are found in U.S. Environmental Protection Agency (1986) test methods for evaluating solid waste, physical/chemical methods SW-846.

${ }^{3} \mathrm{~S}$, soil samples; GW, ground-water samples, SW, surface-water samples; BM, streambed-sediment samples.

\section{Surface-Water and Streambed-Sediment Sampling}

Surface-water and streambed-sediment sampling locations were chosen to monitor flow onto and off of the Plant. The seven sampling sites are shown in figures 10 and 11 (at back of report). Mason's Run (fig. 10) is channeled with a concrete culvert through most of its extent within the Plant boundaries. It drains from the Port Columbus Airport, flows underground southward through the facility, and reemerges and exits the Plant entrance under Fifth Avenue. Turkey Run (fig. 11) is an intermittent stream that crosses the westernmost segment of the Plant after passing through Port Columbus Airport. Approximately 375 feet of Turkey Run traverses the Plant property. An extensive onsite stormwater drainage system has been constructed throughout the plant property and drains into Mason's Run in the central part of the Plant, whereas some stormwater from the western part of the Plant drains into Turkey Run.

Surface-water and streambed-sediment samples were collected from the same sampling location, the surface-water sample being done first to minimize the collection of unnaturally suspended streambed sediments. Surface-water samples were collected as a grab sample from one point in the stream and put directly into the pre-preserved containers. Streambed-sediment samples were collected using a stainless steel scoop, transferred to a stainless steel bowl, and composited 
using the scoop. Sample jars were filled with the composited material from the bowl. Samples for VOC analysis were not composited but were grabbed directly from the streambed. Field measurements of specific conductance, $\mathrm{pH}$, temperature, and alkalinity were made directly from the surface-water body and are on file at the USGS office in Columbus, Ohio.

After collection, the surface-water and streambed-sediment samples were chilled and shipped by overnight courier to a contract laboratory for analysis. Analyses for surface-water and streambed-sediment samples are listed in table 2 .

\section{Quality-Assurance and Quality-Control Measures}

Many measures were implemented by the USGS during data collection to ensure that the appropriate levels of quality assurance and quality control $(\mathrm{QA} / \mathrm{QC})$ were achieved. These measures served to ensure quality, precision, accuracy, completeness, and representativeness of the data generated during the study. The $\mathrm{QA} / \mathrm{QC}$ measures were followed in accordance with the Surface-Water and Ground-Water Monitoring Plan (Parnell, 1996). For all sampling types during the study, 100 percent of the analytical data generated by the laboratory were validated to determine completeness of the data-package deliverables and achievement of the specific data objectives. An independent environmental firm was contracted by the USGS to perform the data validation; a summary of the data-validation process is presented in the Technical Memorandum for the Surface-Water and GroundWater Monitoring Plan (Parnell, 1997). The results of samples analyses for soil, ground-water, surface-water, streambed sediment, and QA/QC samples are presented in Appendix 3.

\section{GEOLOGY AND HYDROLOGY}

The Plant lies within the Till Plains section of the Central Lowland Physiographic Province (Fenneman, 1938). Till Plains were formed through glaciation and burial of preglacial topography. Soils belonging to the Bennington-Pewamo Association are present at AFP 85 (U.S. Department of Agriculture, 1977). The soils range from a yellowish-brown silty loam to a gray clay loam and are generally wet to ponded, easily eroded, and poorly permeable.
Glacial till interbedded with glacial outwash is the predominant surficial material at AFP 85 (fig. 12, at back of report). The glacial outwash is composed of fine, well-sorted sand and gravel-sized material, whereas the glacial till is unstratified, poorly sorted material composed of a mixture of clay, sand, gravel, and various sizes of boulders (Battelle Denver Operations, 1989). Glacial tills and outwash deposits were cut by more recent outwash channels, resulting in complex interbedding and lateral and vertical facies changes within relatively short distances. This glacial interfingered outwash and till results in laterally and horizontally discontinuous water-bearing zones beneath the Plant but does not form a continuous aquifer (Battelle Denver Operations, 1989). Geologic logs of the boreholes indicate that the glacial till ranges in thickness from 10 to 85 feet and that average thickness is about 25 feet across the Plant. It has been reported that a major tributary to the main buried channel of a preglacial river flowed beneath what is now the southern boundary of AFP 85 (Battelle Denver Operations, 1989). Drill logs (USB04 and USB20) in this area, however, indicate that the lithology is clay with some gravel and cobbles to the top of the shale bedrock, not indicative of a buried channel. The preglacial river noted in the same report was reported to cut a channel approximately 200 feet below land surface. Logs of the new borings in this area (USB04 and USB 15), however, indicate that the depth to the shale bedrock was 83 feet and 95 feet, respectively.

Three major aquifer systems are present in Franklin County: Devonian limestone aquifers, Mississippian sandstone aquifers, and glacial aquifers. Thin, discontinuous, glacial water-bearing units underlie AFP 85, as described above. Well yields in the glacial system are as much as 2 gallons per minute from the two water-bearing zones underlying the Plant (U.S. Air Force Aeronautical Systems Center, 1994).

Isolated water-bearing zones also lie within till as a discontinuous basal deposit of coarse sand and gravel just above the shale bedrock. This discontinuous unit is of varied thickness, according to information in drilling logs. Beneath the glacial till at AFP 85 lies the Ohio Shale, which is generally considered to be a poorly permeable unit. This black to dark-brown, sandy, fissile shale has a high organic content.

The gross lithologies recorded for previous wells and borings at the Plant are similar to those found in the new borings, but proportions of clays, sands, and gravels differ greatly from area to area. Figure 13 (at back 
of report) shows a cross section of monitoring wells across the Plant and its variability in geology. The variability in lithologies and the discontinuity between sand and gravel lenses makes mapping of any specific unit extremely difficult. Because of this heterogeneity of the unconsolidated deposits, no attempt was made to interpolate the stratigraphy between the wells and borings.

Infiltration from creeks and streams may recharge the water-bearing units; however, much of the surface water is collected and diverted by tile drainage into concrete channels in and around the Plant.

\section{SOIL DATA}

Two soil samples were collected at selected intervals during drilling and one sample was taken at each of the three hand-auger borings. Forty-nine regular/environmental soil samples and nine QA/QC samples were collected and analyzed.

\section{Volatile Organic Compounds}

The only unqualified VOC detection was $1,1,1$-trichloroethane at USB12 $(0.0099 \mathrm{mg} / \mathrm{kg})$, whereas detections in 13 other samples were qualified. Acetone and methylene chloride were found in method blanks and not considered. The qualified VOC data are listed in the following table. ("Unqualified" data are those that require no qualifier after data validation, whereas "qualified" data are subject to qualification after validation.) A list of validated VOC's found in the soil is given in Appendix 3.

\begin{tabular}{llccc}
\hline Location & \multicolumn{1}{c}{ Analyte } & $\begin{array}{c}\text { Concentration } \\
(\mathbf{m g} / \mathbf{k g})\end{array}$ & $\begin{array}{c}\text { Sample } \\
\text { depth (ft) }\end{array}$ & $\begin{array}{c}\text { Data } \\
\text { qualifier }\end{array}$ \\
\hline USB02 & Tetrachloroethene & 0.0056 & 30 & $\mathrm{~J}$ \\
USB02 & Toluene & 0.0069 & 30 & $\mathrm{~J}$ \\
USB03 & 2-Butanone & 0.047 & 4 & $\mathrm{~J}$ \\
USB05 & Benzene & 0.097 & 12 & $\mathrm{~J}$ \\
USB05 & Vinyl chloride & 0.014 & 12 & $\mathrm{~J}$ \\
USB05 & Benzene & 0.0091 & 12 & $\mathrm{~J}$ \\
USB05 & Ethylbenzene & 0.0051 & 12 & $\mathrm{~J}$ \\
USB05 & Xylenes & 0.015 & 12 & $\mathrm{~J}$ \\
USB06 & Benzene & 0.0088 & 9 & $\mathrm{~J}$ \\
USB07 & Carbon disulfide & 0.0055 & 4 & $\mathrm{~J}$ \\
USB08 & 2-Butanone & 0.078 & 4 & $\mathrm{~J}$ \\
USB09 & 2-Butanone & 0.037 & 2 & $\mathrm{~J}$
\end{tabular}

\begin{tabular}{llccc}
\hline Location & \multicolumn{1}{c}{ Analyte } & $\begin{array}{c}\text { Concentration } \\
(\mathbf{m g} / \mathbf{k g})\end{array}$ & $\begin{array}{c}\text { Sample } \\
\text { depth (ft) }\end{array}$ & $\begin{array}{c}\text { Data } \\
\text { quaifier }\end{array}$ \\
\hline USB1302 & Toluene & 0.041 & 2 & $\mathrm{~J}$ \\
USB1302 & Xylene & 0.0054 & 2 & $\mathrm{~J}$ \\
USB15 & 2-Butanone & 0.022 & 19 & $\mathrm{~J}$ \\
USB15 & Toluene & 0.010 & 19 & $\mathrm{~J}$ \\
USB18A & 2-Butanone & 0.065 & 2 & $\mathrm{~J}$ \\
USB18B & Toluene & 0.0089 & 2 & $\mathrm{~J}$ \\
USB18C & Toluene & 0.0088 & 2 & $\mathrm{~J}$ \\
USB19 & Toluene & 0.0090 & 27 & $\mathrm{~J}$ \\
\hline \multicolumn{2}{c}{ 1 J, estimated value. } & & &
\end{tabular}

\section{Semivolatile Organic Compounds}

Unqualified semivolatile organic compounds (SVOC's) were detected in soil samples from five borings, and one qualified detection of sample of bis(2ethylhexyl)phthalate, at $0.35 \mathrm{mg} / \mathrm{kg}$ was found at USB 19; results are given in the following table. A list of validated SVOC's found in the soil is given in Appendix 3.

\begin{tabular}{llcc}
\hline Location & \multicolumn{1}{c}{ Anaiyte } & $\begin{array}{c}\text { Concentration } \\
\text { (mg/kg) }\end{array}$ & $\begin{array}{c}\text { Sample } \\
\text { Depth (ft) }\end{array}$ \\
\hline USB01 & Phenanthrene & 0.40 & 6 \\
USB01 & Fluoranthene & 0.79 & 6 \\
USB01 & Pyrene & 0.78 & 6 \\
USB01 & Chrysene & 0.37 & 6 \\
USB01 & Benzo(k)fluoranthene & 0.41 & 6 \\
USB05 & 2-Methylnaphthalene & 0.61 & 12 \\
USB07 & 2-Methylnaphthalene & 0.42 & 6.5 \\
USB07 & Phenanthrene & 0.40 & 6.5 \\
USB07 & Pyrene & 0.47 & 6.5 \\
USB07 & Fluoranthene & 0.46 & 6.5 \\
USB08 & Napthalene & 0.99 & 4 \\
USB08 & 2-Methylnaphthalene & 1.4 & 4 \\
USB21 & Fluoranthene & 0.61 & 3 \\
USB21 & Phenanthrene & 0.46 & 3 \\
USB21 & Pyrene & 0.57 & 3 \\
\hline
\end{tabular}

\section{Pesticides and Polychlorinated Biphenyls}

Unqualified pesticides and polychlorinated biphenyls (PCB's) were detected in soil samples from nine borings; results are given in the following table. A list of validated pesticides and PCB's found in the soil is given in Appendix 3. 


\begin{tabular}{lllc}
\hline Location & \multicolumn{1}{c}{ Analyte } & $\begin{array}{c}\text { Concentration } \\
(\mathbf{m g} / \mathbf{k g})\end{array}$ & $\begin{array}{c}\text { Sampie } \\
\text { Depth (ft) }\end{array}$ \\
\hline USB01 & Arochlor 1248 & 0.040 & 6 \\
USB01 & Endrin ketone & 0.0091 & 6 \\
USB03 & delta BHC & 0.0058 & 4 \\
USB04 & Heptachlor epoxide & 0.057 & 35 \\
USB05 & Heptachlor epoxide & 0.030 & 12 \\
USB07 & delta BHC & 0.0029 & 6.5 \\
USB07 & Heptachlor epoxide & 0.063 & 6.5 \\
USB08 & Heptachlor epoxide & 0.037 & 9 \\
USB10 & delta BHC & 0.0023 & 14 \\
USB11 & Arochlor 1248 & 0.056 & 2 \\
USB11 & delta BHC & 0.0077 & 2 \\
USB12 & delta BHC & 0.012 & 5 \\
USB12 & delta BHC & 0.051 & 9 \\
\hline
\end{tabular}

\section{Selected Metals and Trace Elements}

Soils contaminated by metals and trace elements are common at many industrial sites in Ohio. The presence of trace elements, however, does not necessarily indicate contamination because all soils contain at least some trace concentrations. Contamination by metals and trace elements are commonly determined by comparing background concentrations from nearby locations to site-specific locations. Background metals concentrations for soils in Ohio were compiled by Cox-Colvin \& Associates (1995); arsenic, barium, chromium, lead, and mercury are listed as common industrial background metals in Franklin County. These metals were also common to AFP 85.

Of the 23 analytes, 11 were detected in all soil sampled: aluminium, barium, calcium, cobalt, chromium, copper, iron, magnesium, manganese, vanadium, and zinc. Other metals - beryllium, potassium, molybdenum, nickel, and lead - were found in more than 70 percent of the samples. Silver, sodium, thallium, antimony, and selenium were below the detection limit in all soil samples. A list of validated selected metals and trace elements found in soil are given in Appendix 3.

\section{GROUND-WATER QUALITY}

Ground water was sampled from six existing and four recently installed monitoring wells. Ten regular ground-water samples and nine QA/QC samples were collected. Ground water is not a source of potable drinking water for the Plant.

\section{Volatile Organic Compounds}

Only one VOC was detected in all water from the 10 wells sampled: benzene, at $1.9 \mathrm{mg} / \mathrm{L}$ in USW02 .

\section{Semivolatile Organic Compounds}

Only one SVOC was detected in all groundwater samples from the 10 well: bis(2-ethylhexyl)phthalate, at $31 \mathrm{mg} / \mathrm{L}$ in PG-201.

\section{Pesticides and Polychlorinated Biphenyls}

Only two unqualified pesticides were detected in all ground-water samples: delta $\mathrm{BHC}$, at $0.088 \mathrm{mg} / \mathrm{L}$ in $P G 201$, and heptachlor expoxide, at $0.070 \mathrm{mg} / \mathrm{L}$ in USW02. All PCB concentrations were below the detection limit.

\section{Selected Metals and Trace Elements}

Of the 23 analytes, 3 were detected in all wells sampled-calcium, magnesium, and manganese. Beryllium, silver, cobalt, copper, nickel, antimony, selenium, vanadium, and thallium concentrations were below the detection limit. Cadmium $(0.0083 \mathrm{mg} / \mathrm{L})$ at PG201 and chromium (1.4 mg/L) at USW16 exceeded the Maximum Contaminant Levels of $0.0050 \mathrm{mg} / \mathrm{L}$ and $0.100 \mathrm{mg} / \mathrm{L}$, respectively. A list of validated selected metals and trace elements in ground water is given in Appendix 3.

\section{Common Anions and Cyanide}

Chloride and sulfate were found in every ground-water sample. Cyanide was selectively sampled for at BLD3, PG-201, USW02, USW16, and USW19. A list of validated common anions and cyanide in ground water is given in Appendix 3. 


\section{SURFACE-WATER QUALITY}

Seven regular surface-water samples and three QA/QC samples were collected and analyzed.

\section{Volatile Organic Compounds}

Unqualified VOC's were detected in surfacewater samples from three sites; concentrations are listed in the following table. A list of validated VOC's found in surface water is given in Appendix 3.

\begin{tabular}{llc}
\hline Location & \multicolumn{1}{c}{ Analyte } & $\begin{array}{c}\text { Concentration } \\
(\mu \mathbf{g} / \mathbf{L})\end{array}$ \\
\hline MR04 & Chloroform & 1.5 \\
MR05 & Chloroform & 1.6 \\
TR02 & 1,2-Dichloroethene & 1.4 \\
TR02 & Trichloroethene & 1.2 \\
\hline
\end{tabular}

\section{Semivolatile Organic Compounds}

Concentrations of SVOC's were below the detection limits in all surface-water samples.

\section{Pesticides and Polychlorinated Biphenyls}

Pesticides and PCB's were below the detection limits in all surface-water samples.

\section{Selected Metals and Trace Elements}

Of the 23 analytes, 6 were detected in all surface water samples — barium, calcium, iron, magnesium, manganese, and sodium. Silver, arsenic, beryllium, cadmium, cobalt, chromium, copper, potassium, molybdenum, nickel, lead, vanadium, thallium, antimony, and selenium were not detected in any sample. A list of validated selected metals and trace elements in surface water is given in Appendix 3.

\section{Common Anions and Cyanide}

Chloride, nitrate, and sulfate were found in the surface-water samples. Cyanide concentrations determined for sites MR1, MR 5, and TR 2 were below the detection limits in all water. A validated list of common ions in surface water is given in Appendix 3.

\section{STREAMBED-SEDIMENT DATA}

Seven regular streambed-sediments samples and five QA/QC samples were collected and analyzed.

\section{Volatila Organic Compounds}

Only two qualified VOC's, flagged as estimated $(\mathrm{J})$, were detected in streambed-sediment samples: concentrations are listed in the following table. No unqualified VOC's were found. (Acetone was flagged because of calibration failure and not is reported here.) A list of validated VOC's found in streambed-sediment samples is presented in Appendix 3.

\begin{tabular}{llcc}
\hline Location & \multicolumn{1}{c}{ Analyte } & $\begin{array}{c}\text { Concentration } \\
\text { (mg/kg) }\end{array}$ & $\begin{array}{c}\text { Data } \\
\text { quallfier }\end{array}$ \\
\hline MR03 & 1,2-Dichloroethene & 0.0056 & $\mathrm{~J}$ \\
MR04 & Toluene & 0.15 & $\mathrm{~J}$ \\
\hline
\end{tabular}

\section{Semivolatile Organic Compounds}

No qualified SVOC's were found. Sixteen unqualified SVOC's were detected at all seven sites. A list of validated SVOC's found in streambed-sediment samples is given in Appendix 3.

\section{Pesticides and Polychlorinated Biphenyls}

Unqualified pesticides were detected in streambed-sediment samples; results are given in the following table. A list of validated pesticides and PCB's found in the streambed sediment samples is given in Appendix 3.

\begin{tabular}{lll}
\hline Location & \multicolumn{1}{c}{ Analyte } & \multicolumn{1}{c}{$\begin{array}{c}\text { Concentration } \\
(\mathbf{m g} / \mathbf{k g})\end{array}$} \\
\hline MR04 & Endrin ketone & 1.0 \\
TR02 & Aldrin & 0.0057 \\
TR02 & Endrin ketone & 0.0039 \\
\hline
\end{tabular}

\section{Selected Metals and Trace Elements}

Of the 23 metals included in the analyte list, 16 were detected in all streambed sediments sampled aluminium, barium, beryllium, calcium, cadmium, cobalt, chromium, copper, iron, magnesium, manganese, molybdenum, nickel, lead, vanadium, and zinc. 
Silver, thallium, antimony, sodium, and selenium concentrations were below the detection limits. A list of selected metals and trace elements in streambed sediments is given in Appendix 3.

\section{CONCLUSIONS}

The Surface-Water and Ground-Water Monitoring Plan for AFP 85 was designed to characterize any ground-water, surface-water, and soil contamination that may exist at AFP 85. The investigation activities done by the USGS included the collection of data during drilling and during sampling of ground water, surface water, and streambed sediments.

This report presents data on the selected inorganic and organic constituents in soil, ground water, surface water, and streambed sediments at AFP 85 . The methods of data collection and analysis are also included. The analytical results of the soil, ground water, surface water, and streambed sediments could aid Aeronautical Systems Center, Environmental Management Directorate, Restoration Division, and its governing regulatory agencies in determining the potential pathways for contaminant migration into the underlying water-bearing zones or nearby surface waters and will provide background information for future remediation studies.

The unconsolidated glacial till deposits at the Plant consist of poorly sorted outwash; very fine to coarse sand and gravel is intermingled with poorly permeable clay and cobbled till. Because of this heterogeneity of the unconsolidated deposits, no attempt was made to interpolate the stratigraphy between the wells and borings.

\section{REFERENCES CITED}

Battele Denver Operations, 1989, Installation Restoration Program Stage 2, informal technical information report: Columbus, Ohio, $22 \mathrm{p}$.

Cox, C.A., and Colvin, G.H., 1995, Investigation of background metal concentrations in Ohio soils: Hillard, Ohio, Cox-Colvin and Associates, Inc. [various pagination].

Fenncman, N.M., 1938, Physiography of castern United States: New York, McGraw-Hill, p. 449-536.
Ohio Environmental Protection Agency, 1995, Technical guidance manual for hydrogeologic investigations and ground water monitoring: Division of Drinking and Ground Waters, $21 \mathrm{p}$.

Parnell, J.M., 1996, Surface-water and ground-water monitoring plan for Air Force Plant 85, Columbus, Ohio: On file at the Columbus office of the U.S. Geological Survey [various pagination].

Parnell, J.M., 1997, Technical memorandum for the surfacewater and ground-water monitoring plan for Air Force Plant 85, Columbus, Ohio: On file at the Columbus office of the U.S. Geological Survey [various pagination].

Schmidt, J.J., and Goldthwait, R.P., 1958, The ground-water resources of Franklin County, Ohio: Ohio Department of Natural Resources Bulletin 30, $24 \mathrm{p}$.

U.S. Air Force Aeronautical Systems Center, 1994, Management action plan: Environmental Restoration Program [various pagination].

U.S. Department of Agriculture, 1980, Soil survey of Franklin County: Soil Conservation Service, p. 7.

U.S. Environmental Protection Agency, 1986, Test methods for evaluating solid waste, physical chemical methods, SW-846 (3rd ed.): Washington, D.C., 88 p. 


\section{APPENDIXES}


APPENDIX 1. LOGS OF BORINGS AT AIR FORCE PLANT 85, COLUMBUS, OHIO, 1996 


$\begin{array}{ll}\text { AIR FORCE PLANT } 85 & \text { Columbus, Ohio }\end{array}$

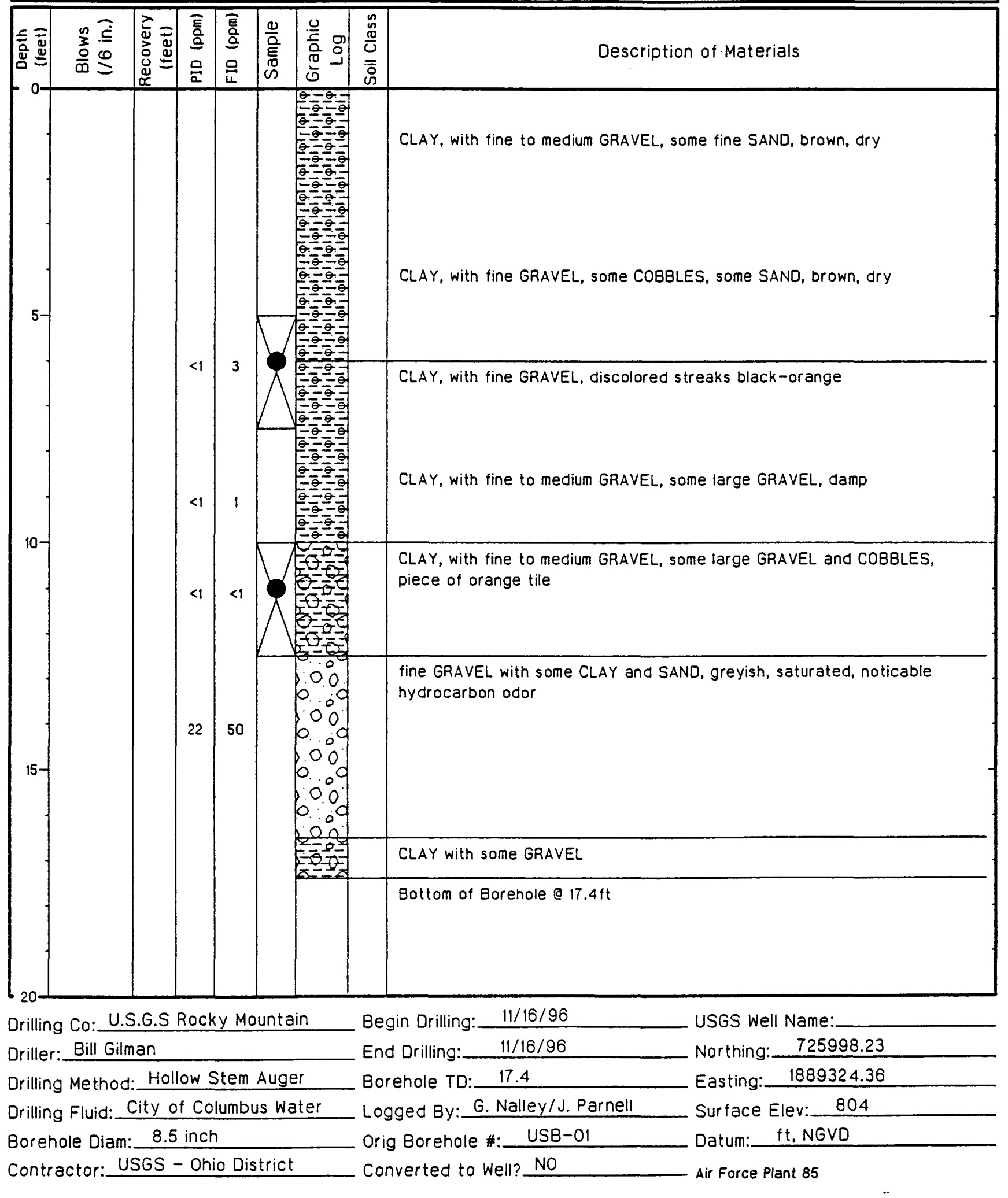




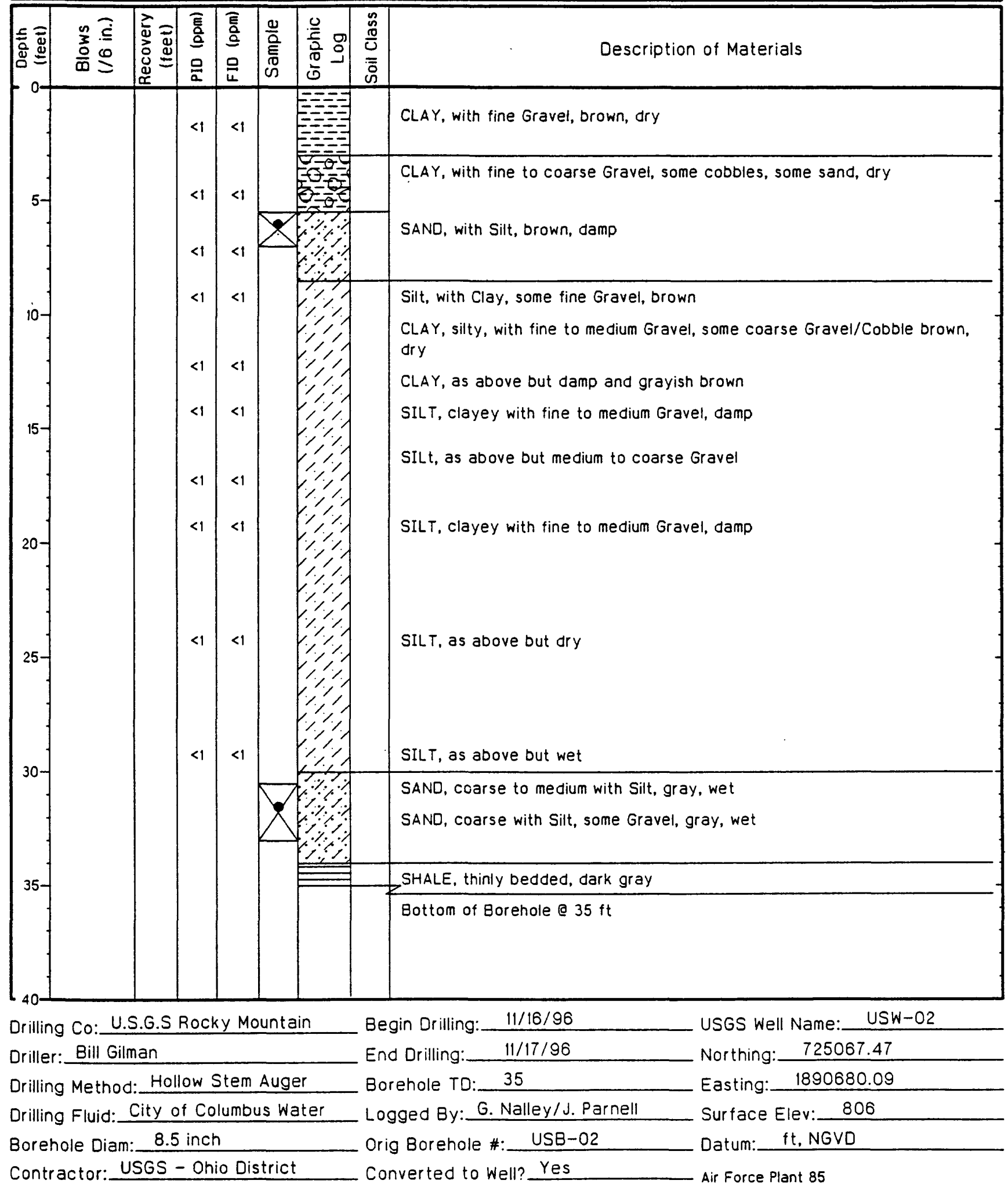




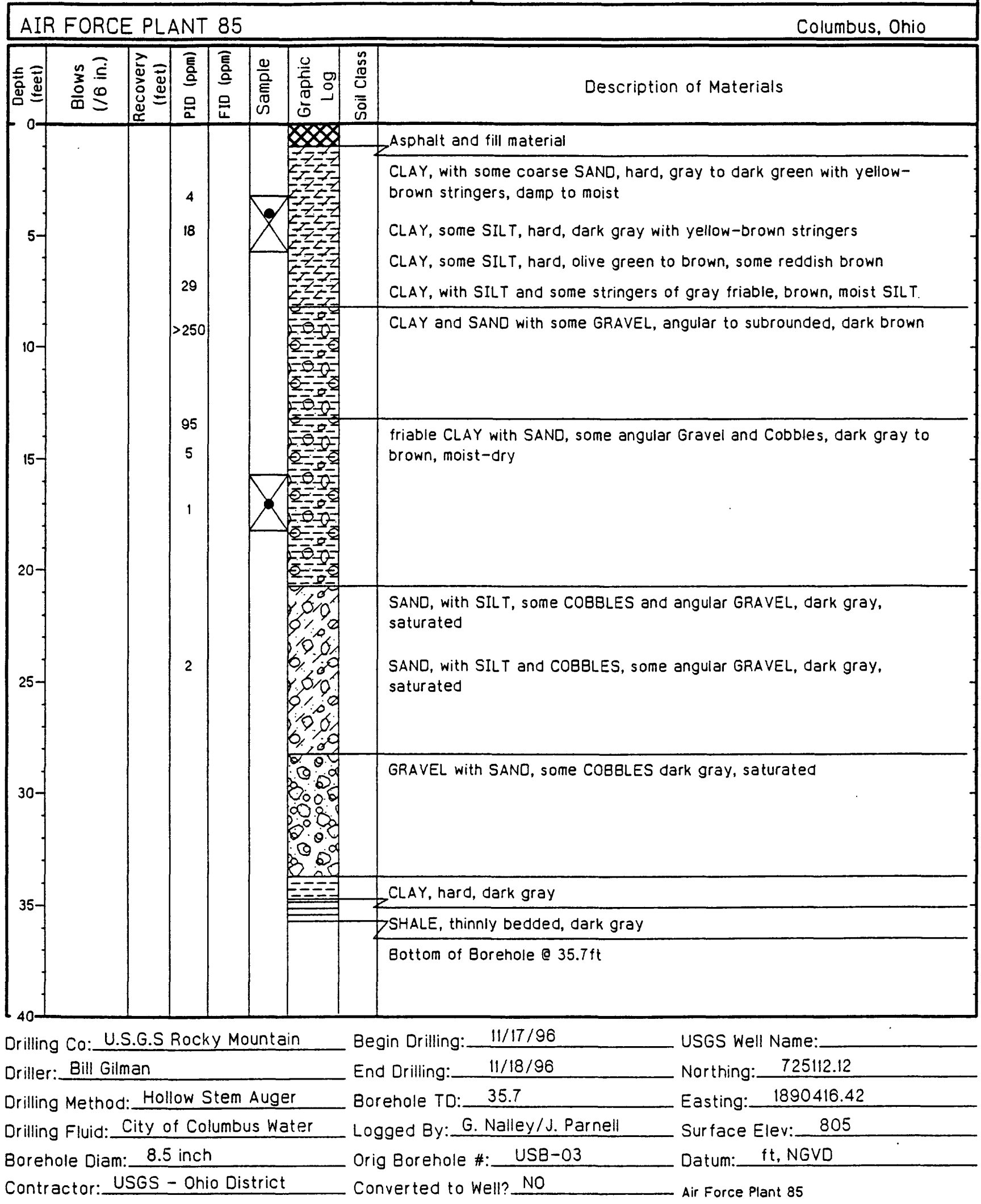




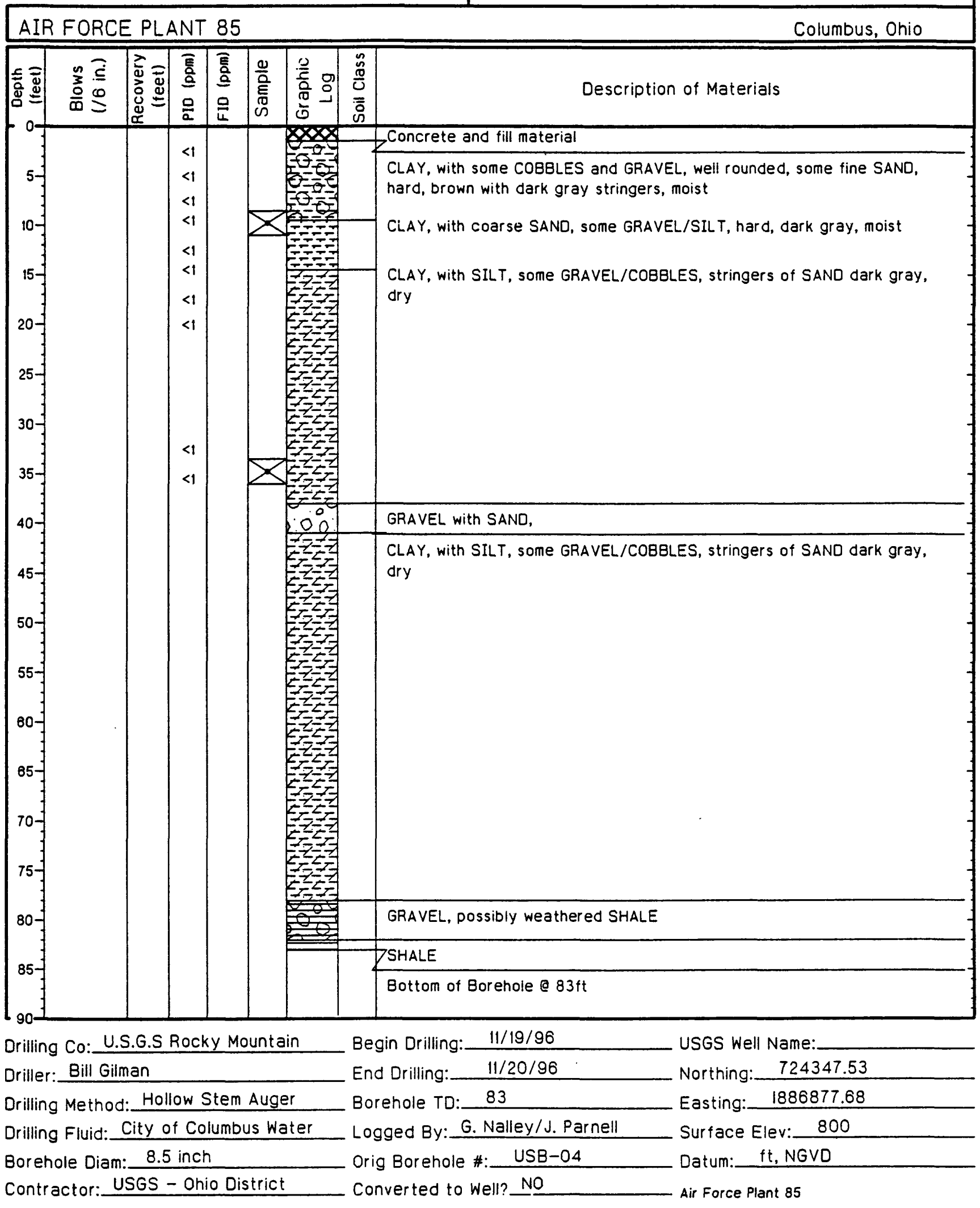




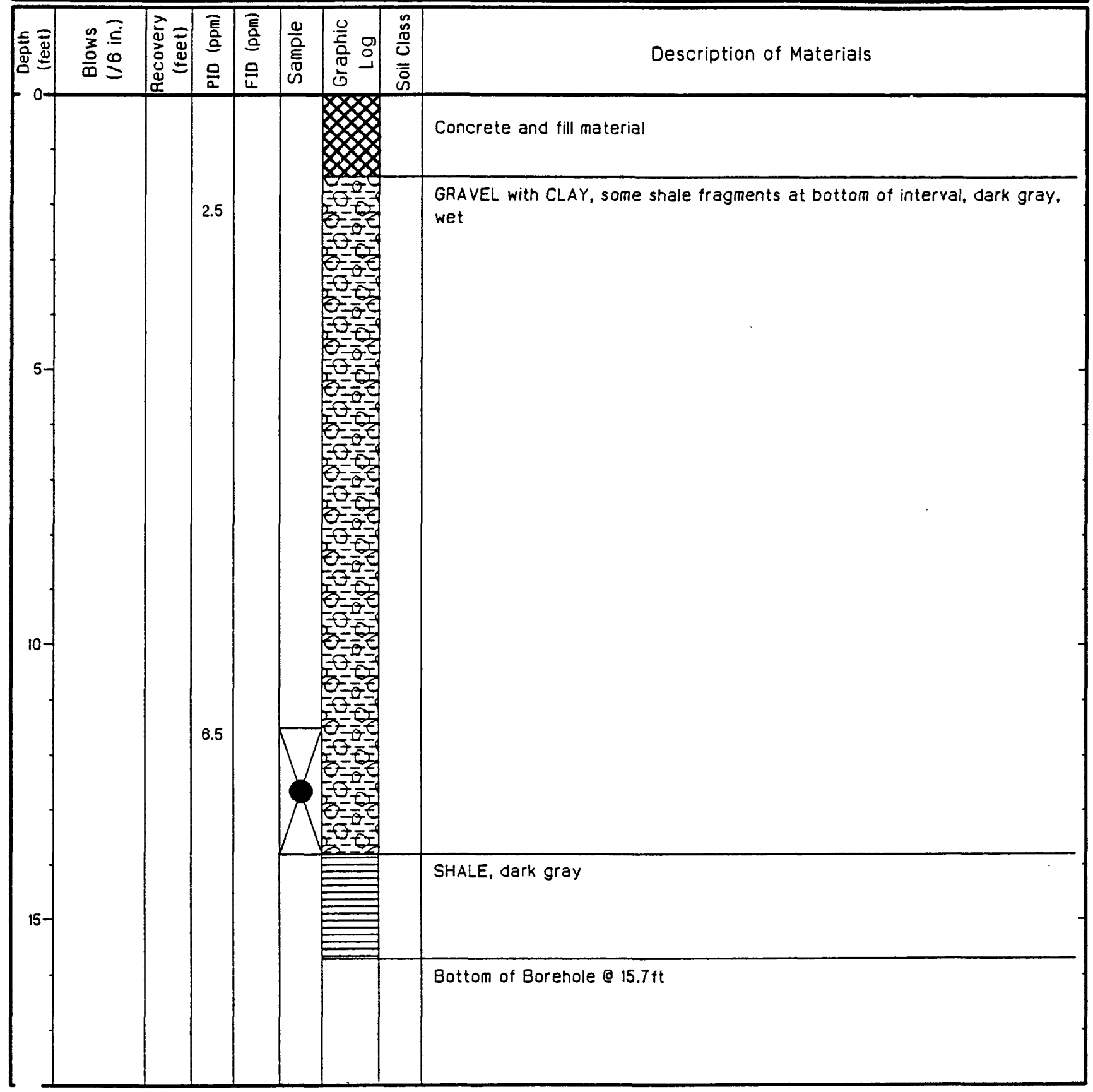

Drilling Co: U.S.G.S Rocky Mountain

Driller: Bill Gilman

Orilling Method: Hollow Stem Auger

Drilling Fluid: City of Columbus Water

Borehole Diam: 8.5 inch

Contractor: USGS - Ohio District
Begin Drilling: $11 / 21 / 96$ End Drilling: $11 / 21 / 96$ Borehole TD: 15.7 Logged By: G. Nalley/J. Parnell Orig Borehole \#: USB-05 Converted to Well? NO
USGS Well Name:

Northing: 724918.66

Easting: 1892346.72

Surface Elev: 808

Datum: $f t, N G V D$

Air Force Plant 85 


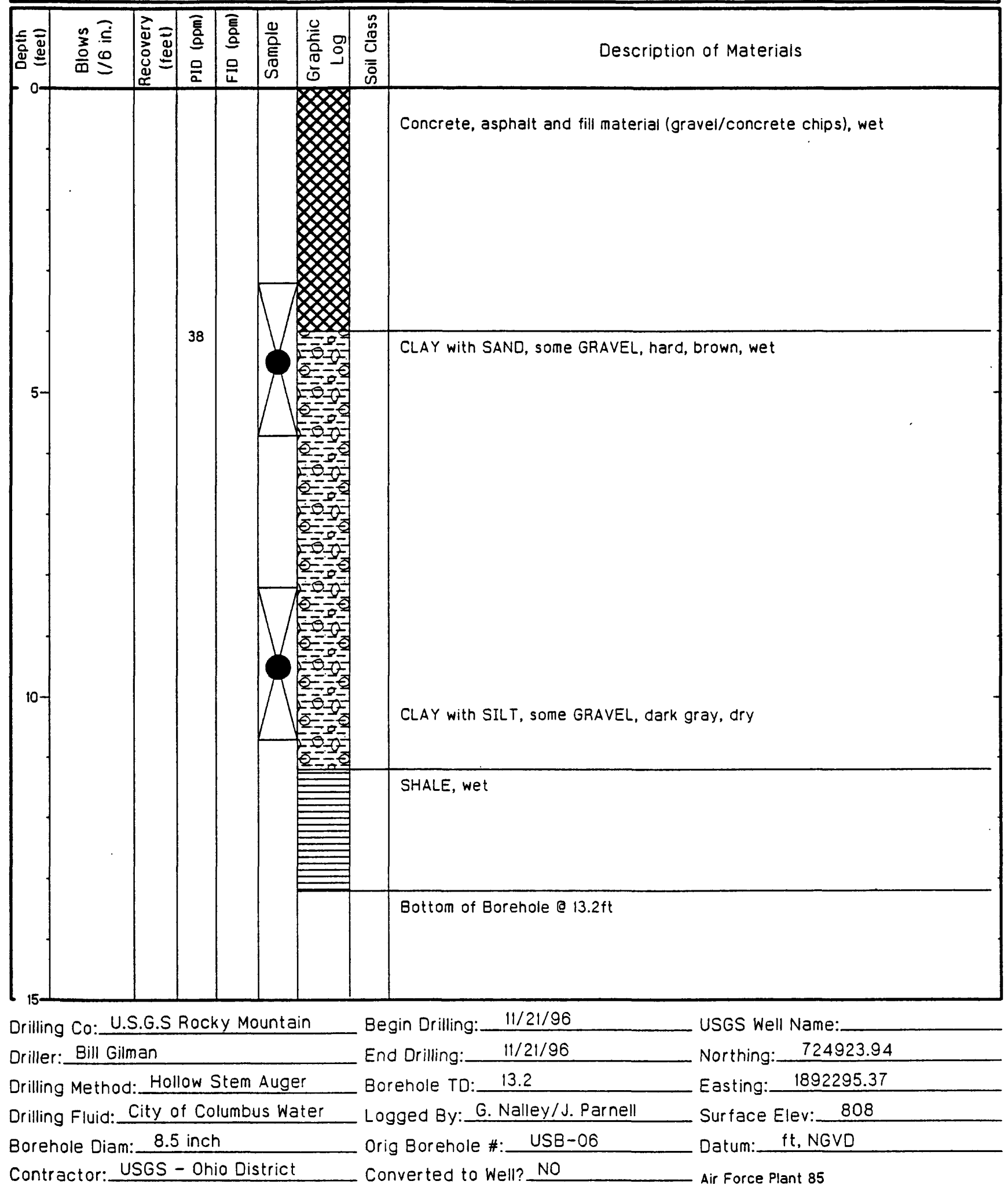




\begin{tabular}{ll}
\hline AIR FORCE PLANT 85 & Columbus, Ohio
\end{tabular}

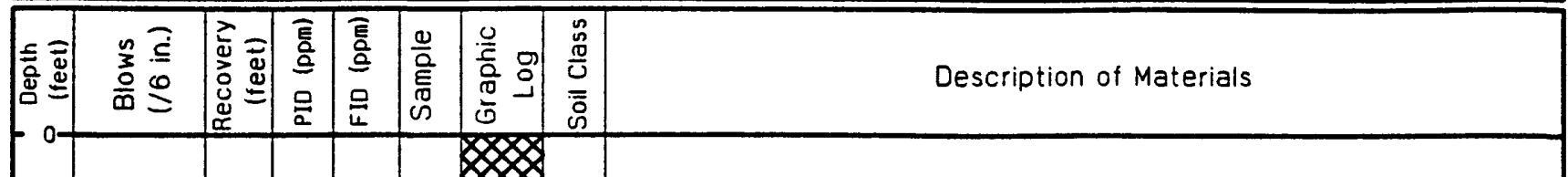

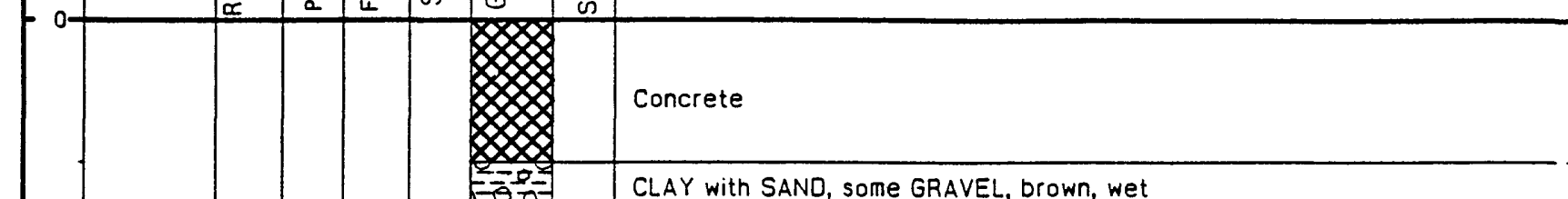

CLAY with SAND, some GRAVEL, gray, wet

CLAY with COBBLE, GRAVEL and SAND, brown

CLAY With SAND, some Snal GRAVE, dark graY

Bottom of Borehole $013.2 \mathrm{ft}$

Drilling Co:U.S.G.S Rocky Mountain

Driller: Bill Gilman

Drilling Method: Hollow Stem Auger

Drilling Fluid: City of Columbus Water

Borehole Diam: 8.5 inch

Contractor: USGS - Ohio District
Begin Drilling:_ II/21/96

End Drilling: $11 / 21 / 96$

Borehole TD: 8

Logged By: G. Nalley/J. Parnell

Orig Borehole \#: USB-07

Converted to Well? NO
USGS Well Name:

Northing: 725032.08

Easting: 1892088.00

Surface Elev: 807

Datum: $f t, N G V D$

Air Force Plant 85 


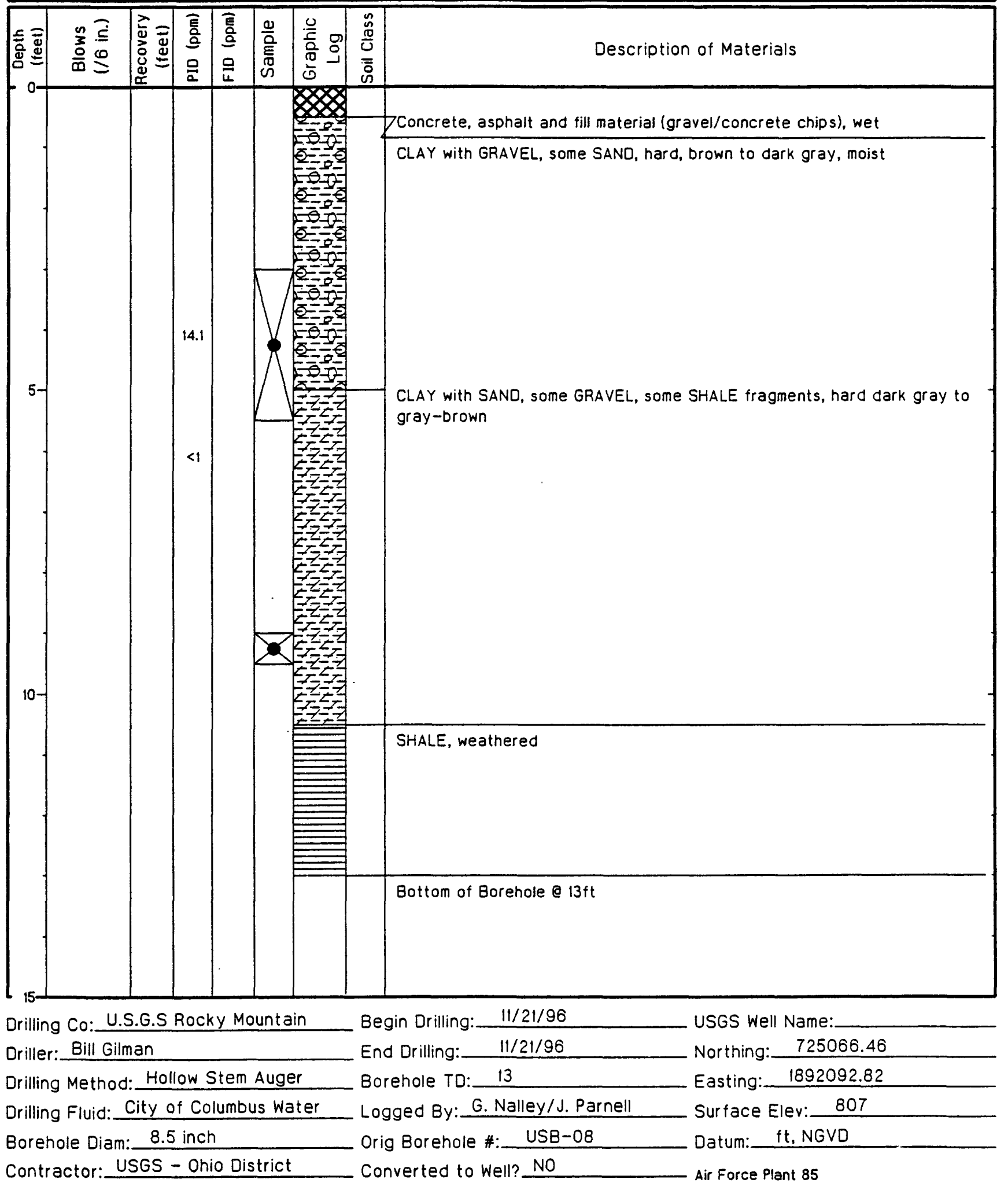




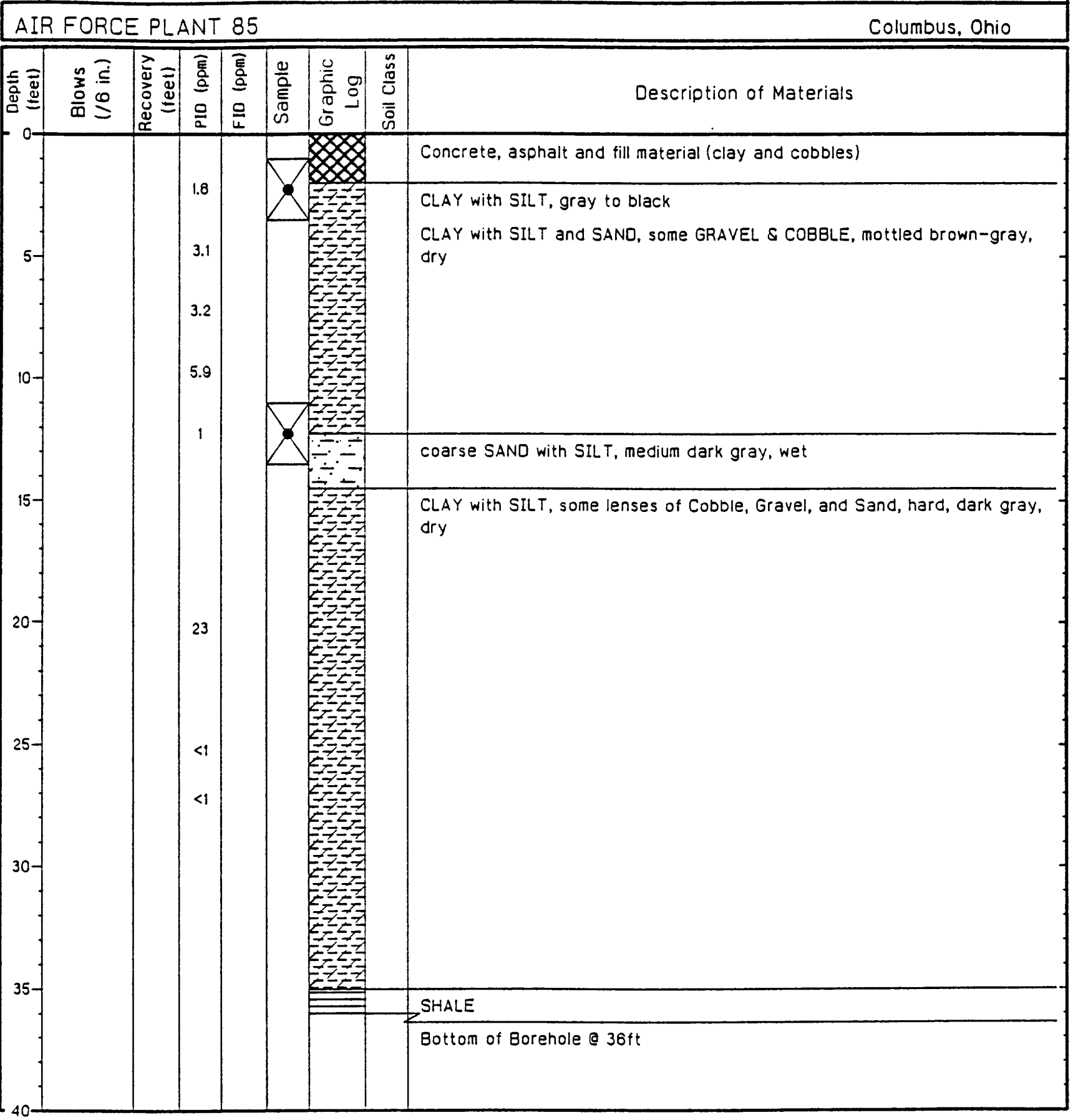

Drilling Co: U.S.G.S Rocky Mountain

Driller: Bill Gilman

Drilling Method: Hollow Stem Auger

Drilling Fluid: City of Columbus Water

Borehole Diam: 8.5 inch

Contractor: USGS - Ohio District
Begin Drilling: $11 / 22 / 96$

End Drilling:_ 11/22/96

Borehole TO: 36

Logged By: G. Nalley/J. Parnell

Orig Borehole \#: USB-OS

Converted to Well? NO
USGS Well Name:

Northing: 726041.22

Easting: 1890840.75

Surface Elev: 806

Datum: $f t, N G V D$

Air Force Plant 85 


\begin{tabular}{|ll}
\hline AIR FORCE PLANT 85 & Columbus, Ohio \\
\hline
\end{tabular}

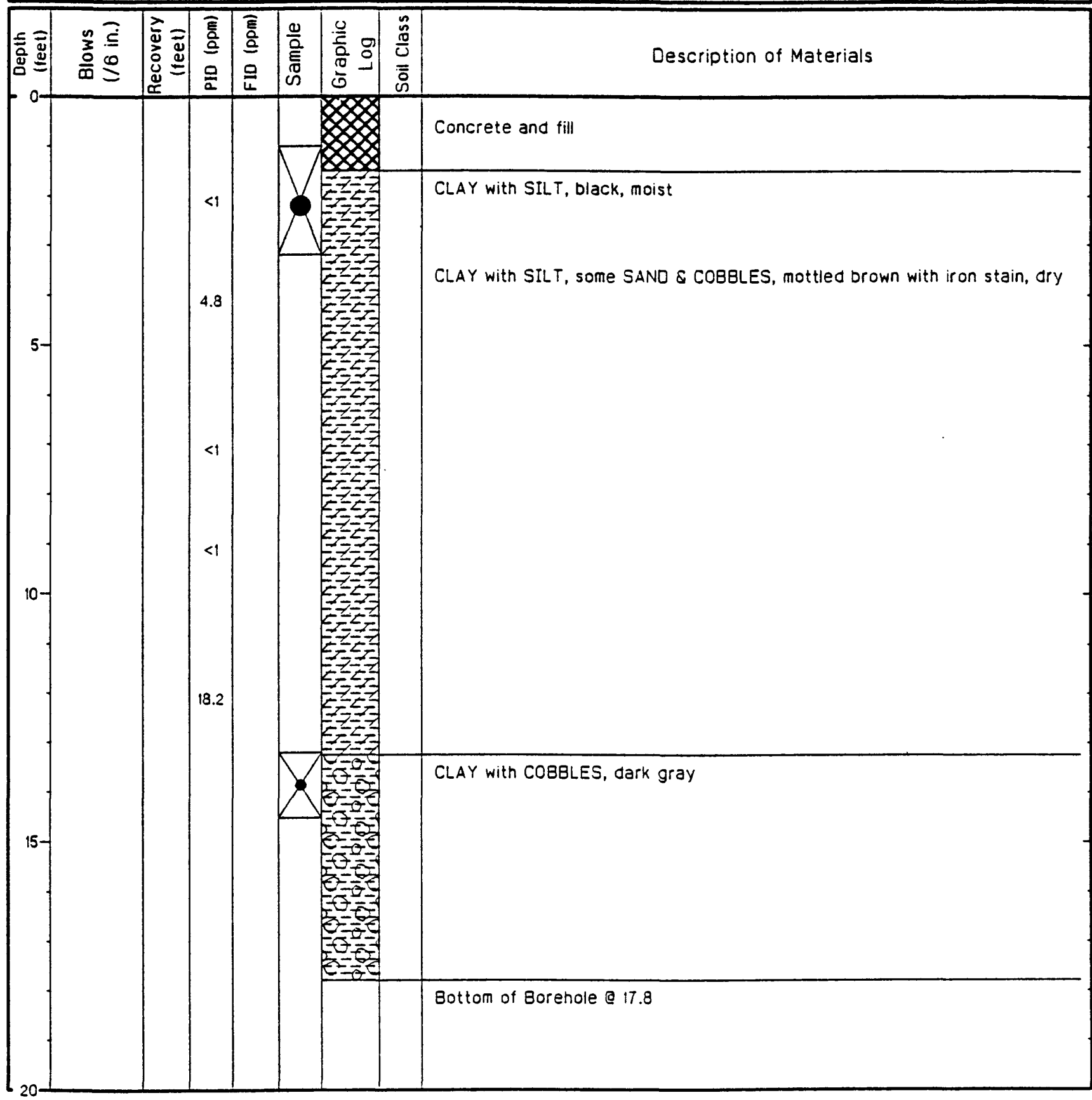

Drilling Co:U.S.G.S Rocky Mountain Driller: Bill Gilman

Drilling Method: Hollow Stem Auger Drilling Fluid: City of Columbus Water Borehole Diam: 8.5 inch Contractor: USGS - Ohio District
Begin Drilling: $11 / 23 / 96$ End Drilling: $11 / 23 / 96$ Borehole TD: 17.8 Logged By: G. Nalley/J. Parnell Orig Borehole \#: USB-10 Converted to Well? NO
USGS Well Name: Northing: 725448.21 Easting: 1890150.12 Surface Elev: 805 Datum: $f t, N G V D$ Air Force Plant 85 


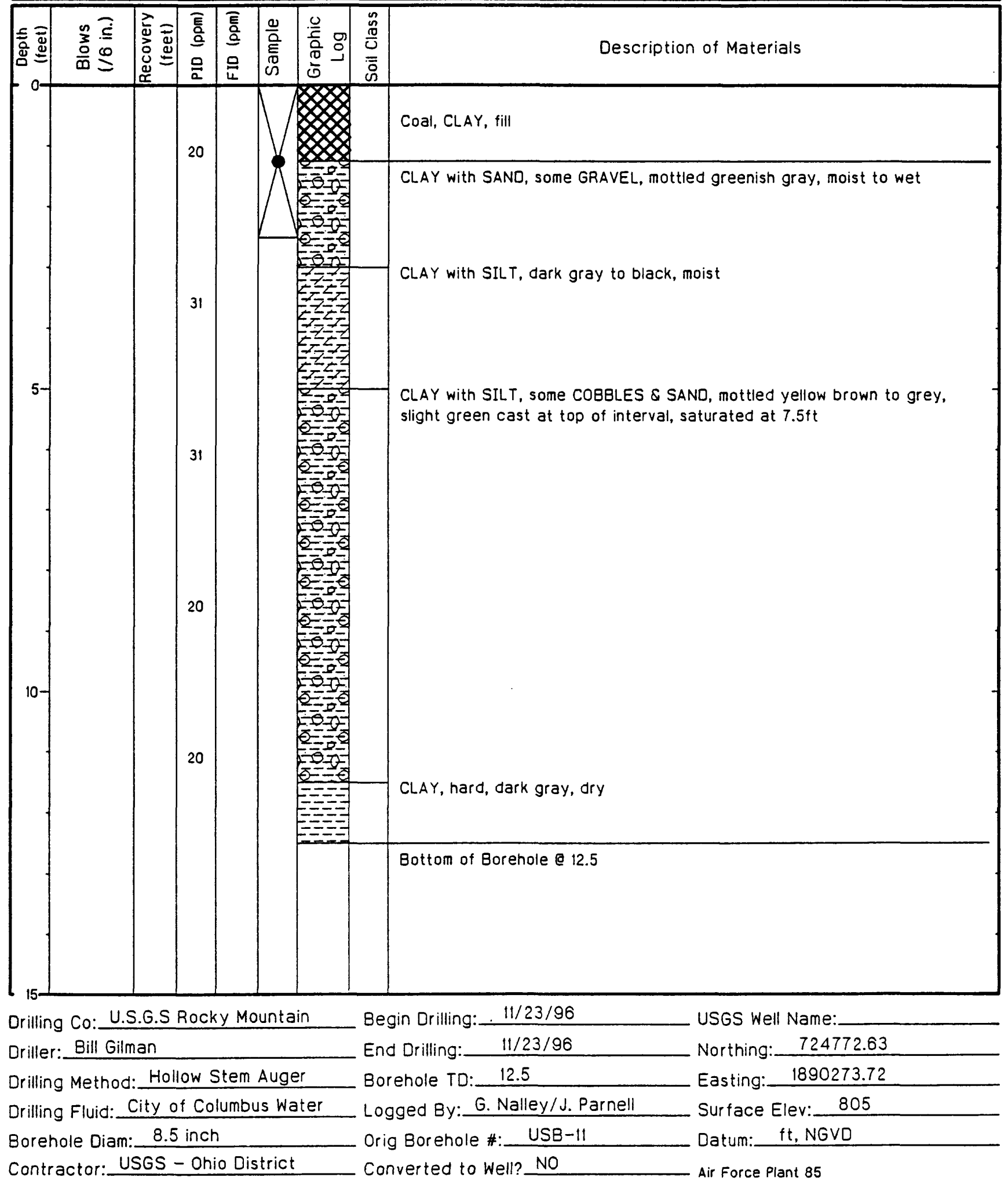




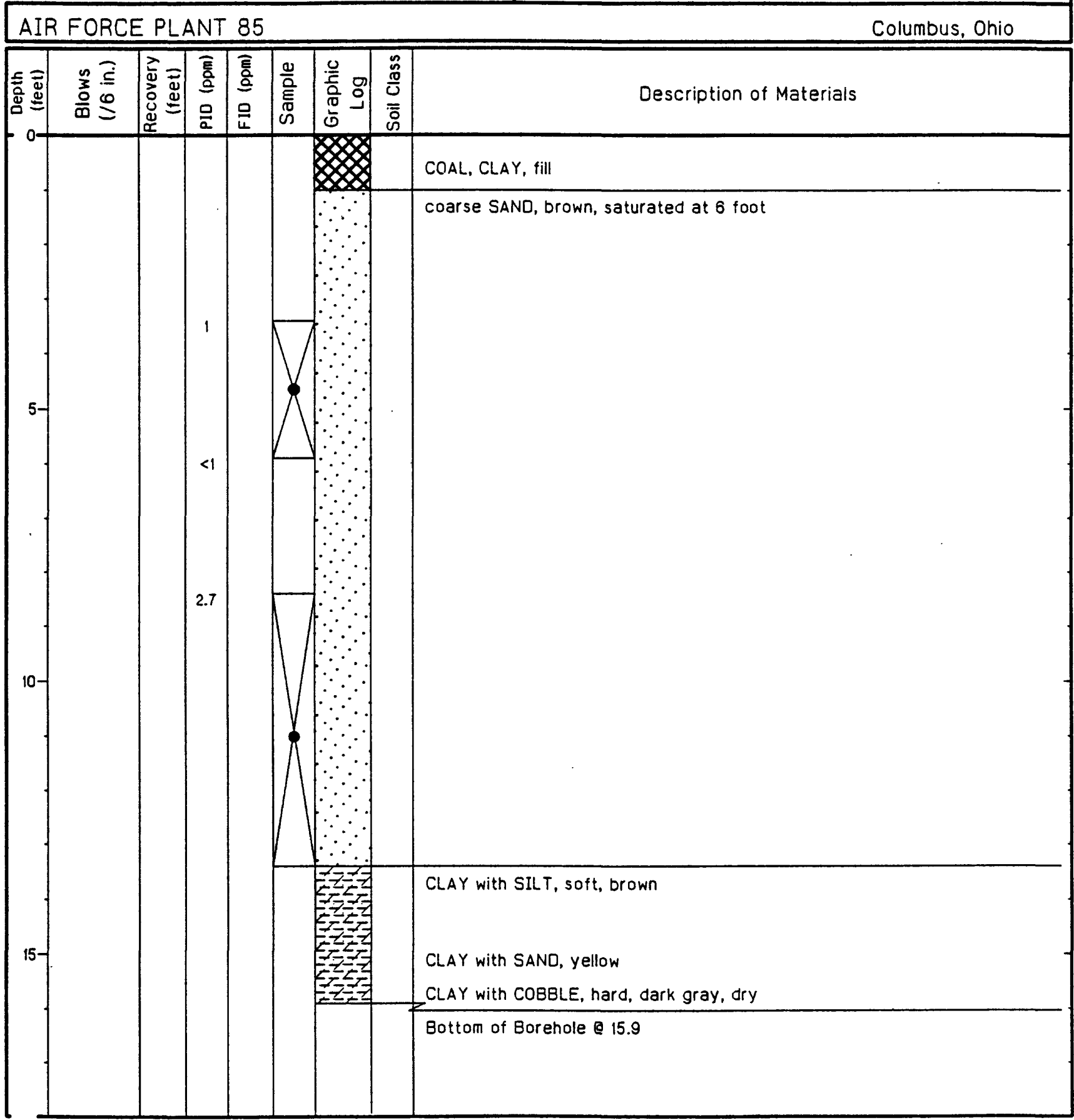

Drilling Co: U.S.G.S Rocky Mountain

Driller: Bill Gilman

Drilling Method: Hollow Stem Auger

Drilling Fluid: City of Columbus Water

Borehole Diam: 8.5 inch

Contractor: USGS - Ohio District
Begin Drilling: $11 / 24 / 96$ End Drilling: $\quad 11 / 24 / 96$ Borehole TO: 15.9 Logged By: G. Nalley/J. Parnell Orig Borehole \#: USB-12 Converted to Well? NO USGS Well Name: Northing: 724511.05 Easting: 1890332.94 Surface Elev: 805 Datum: ft, NGVD Air Force Plant 85 


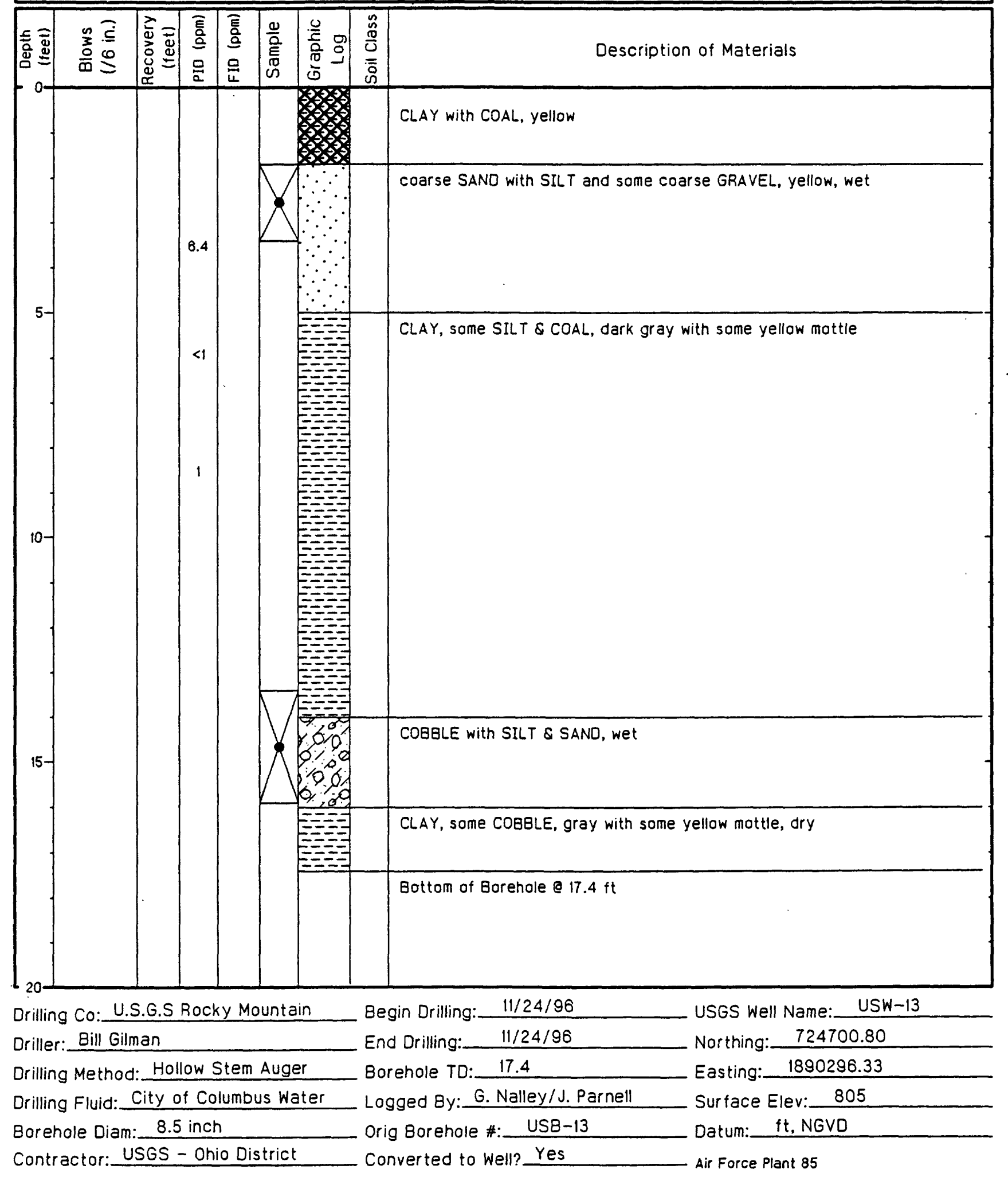




AIR FORCE PLANT $85 \quad$ Columbus, Ohio

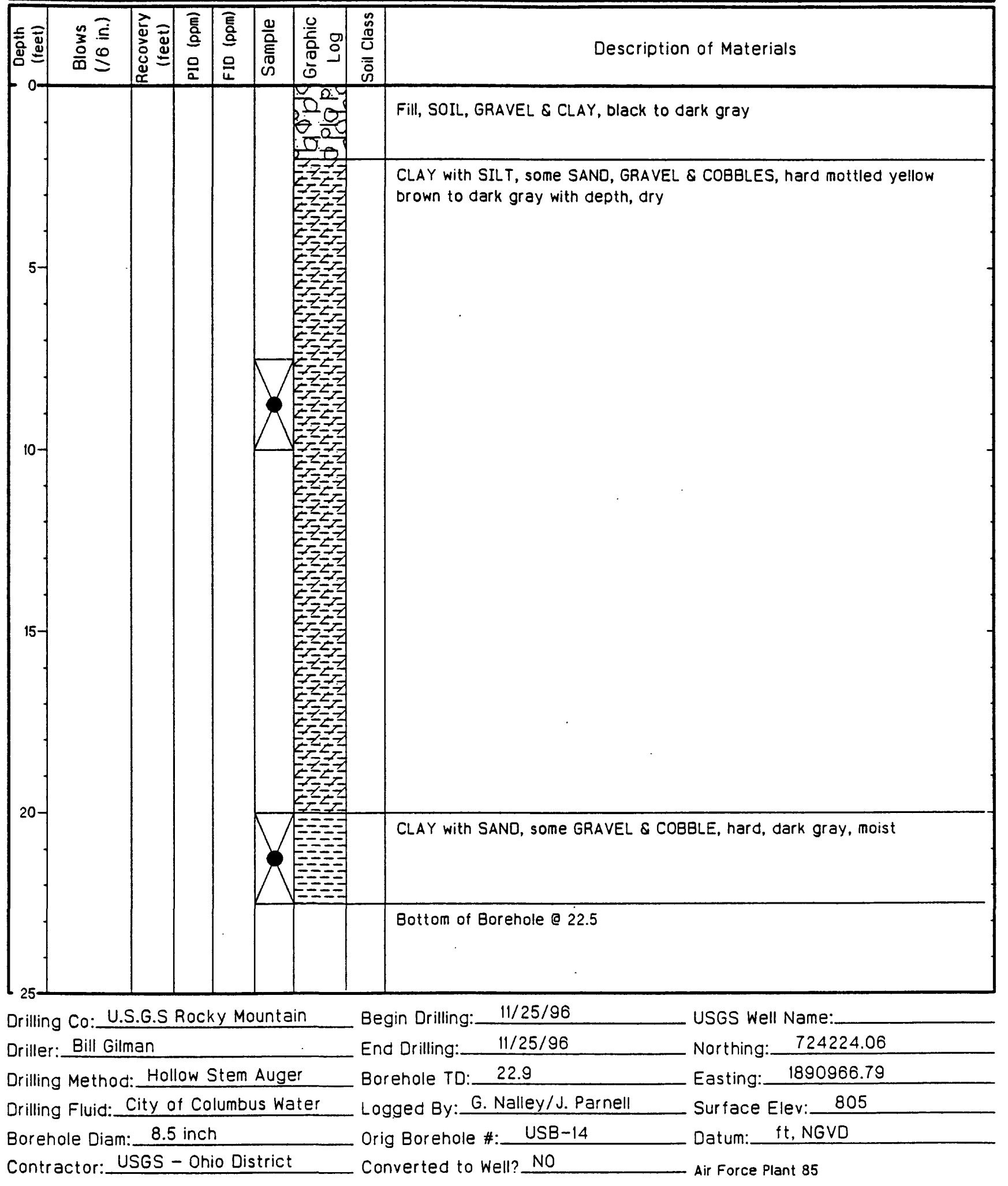




\begin{tabular}{|c|c|c|c|c|c|c|}
\hline & & \\
\hline
\end{tabular}

Drilling Co: U.S.G.S Rocky Mountain

Oriller: Bill Gilman

Drilling Method: Hollow Stem Auger

Drilling Fluid: City of Columbus Water

Borehole Diam: 8.5 inch

Contractor: USGS - Ohio District
Begin Drilling: $12 / 3 / 96$

End Drilling:

$12 / 3 / 96$

Borehole TD: $\$ 5$

Logged By: G. Nalley/J. Parnell

Orig Borehole \#: USB-15

Converted to Well? NO
USGS Well Name:

Northing: 725370.29

Easting: 1886257.23

Surface Elev: 800

Datum: $f t, N G V D$

Air Force Plant 85 


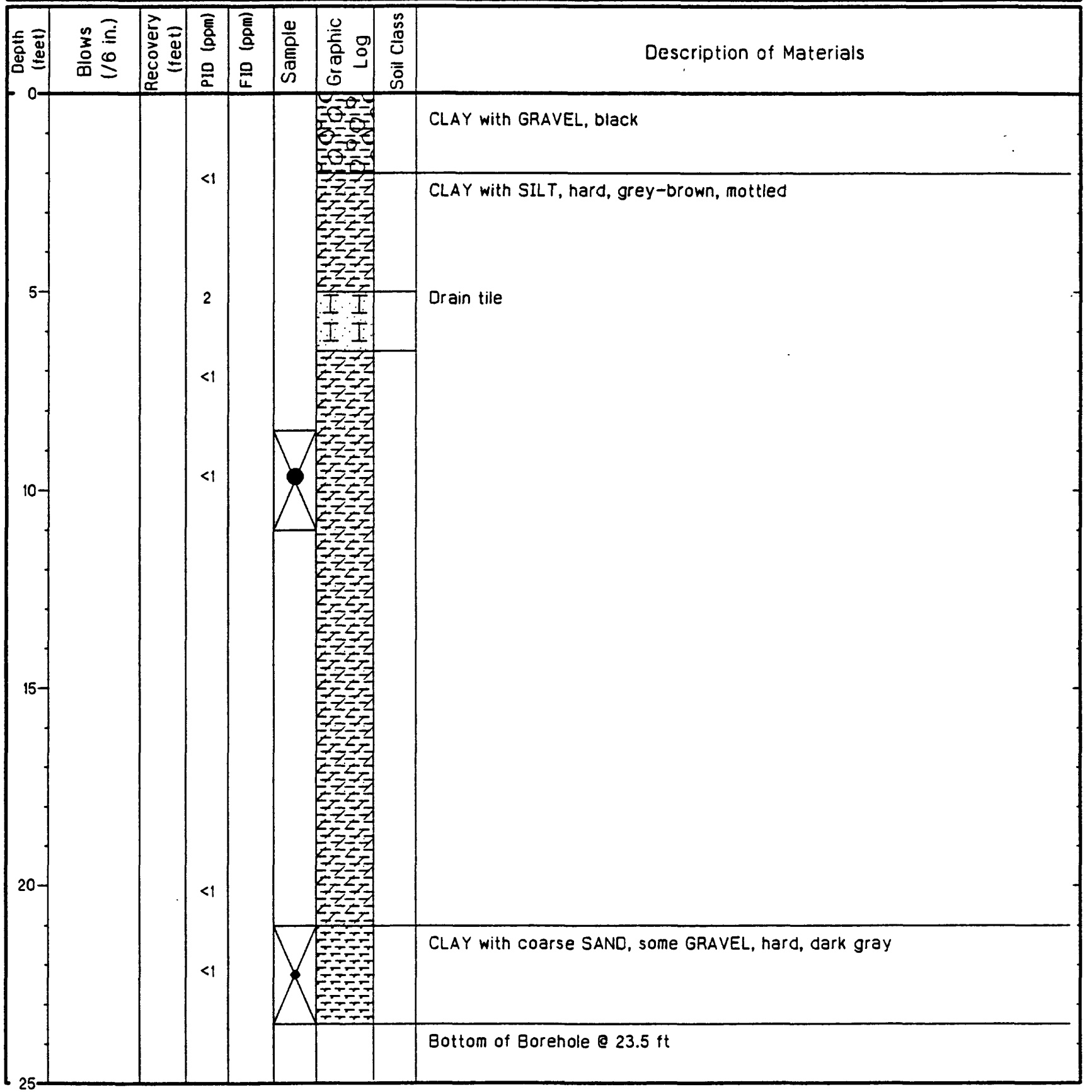

\begin{tabular}{|c|c|c|}
\hline Drilling Co: U.S.G.S Rocky Mountain & Begin Drilling:_ & USGS Well Name: \\
\hline Driller: Bill Gilman & $12 / 04 / 96$ & Northing: $\quad 725350.85$ \\
\hline Drilling Method: Hollow Stem Auger & Borehole TD: & 1886531.12 \\
\hline Drilling Fluid: City of Columbus Water & Logged By: G. Nalley/J. Parnell & Surface Elev: \\
\hline Borehole Diam: 8.5 inch & Orig Borehole \#: USB-16 & Datum: $\mathrm{ft}, \mathrm{NGVD}$ \\
\hline Contractor: USGS - Ohio District & Converted to Well? Yes & Air Force Plant 85 \\
\hline
\end{tabular}




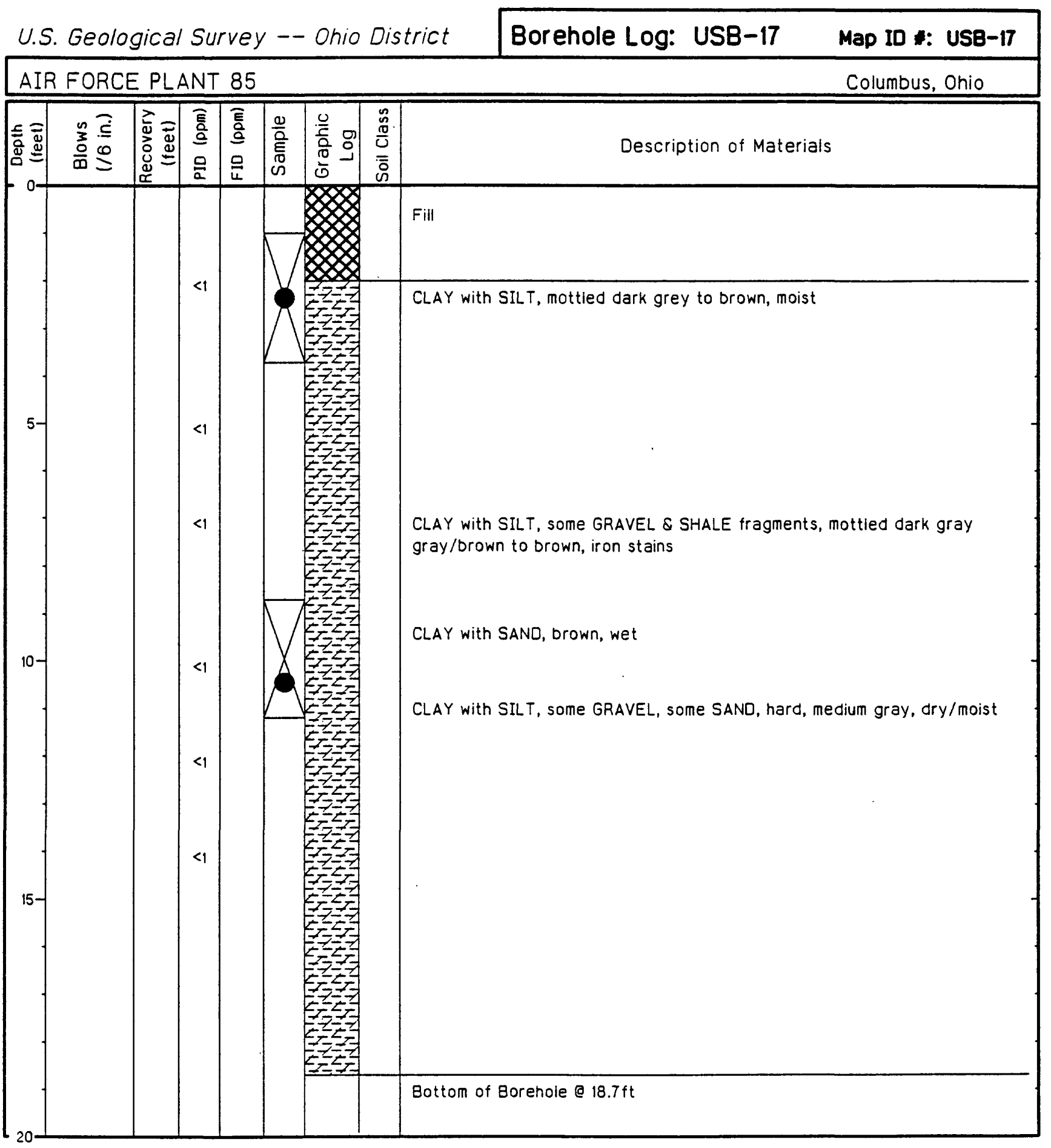

\begin{tabular}{|c|c|c|}
\hline Drilling Co: U.S.G.S Rocky Mountain & Begin Drilling: $\quad 12 / 5 / 96$ & USGS Well Name: \\
\hline Driller:Bill Gilman & End Drilling:_ & Northing: $\quad 725224.12$ \\
\hline Drilling Method: Hollow Stem Auger & Borehole TD: 18.7 & Easting: $\quad 1886360.87$ \\
\hline Drilling Fluid: City of Columbus Water & Logged By: G. Nalley/J. Parnell & Surface Elev: 801 \\
\hline Borehole Diam: 8.5 inch & Orig Borehole \#: USB-17 & Datum: ft, NGVD \\
\hline Contractor: USGS - Ohio District & Converted to Well? NO & Air Force Plant \\
\hline
\end{tabular}




\begin{tabular}{|c|c|c|c|c|c|c|c|c|}
\hline $\begin{array}{l}\frac{E}{\bar{a}} \\
\overline{\mathrm{a}} \\
\mathrm{g}\end{array}$ & 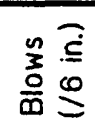 & 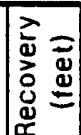 & $\begin{array}{l}\overline{\bar{E}} \\
\text { a } \\
\text { a }\end{array}$ & $\begin{array}{l}\bar{E} \\
\text { 을 } \\
\text { 은 }\end{array}$ & 焉 & $\begin{array}{l}0 \\
\frac{\pi}{0} \\
\frac{0}{0}\end{array}$ & $\begin{array}{l}\text { D. } \\
\frac{0}{0} \\
0 \\
\overline{\overline{0}}\end{array}$ & Description of Materials \\
\hline
\end{tabular}

Fill (clay sand, gravel, asphalt, cement), dry

E=E- $\quad$ CLAY, some SILT, dark brown, moist

Bottom of Borehole $03.1 \mathrm{tt}$

Dritling Co:

Oriller:

Drilling Method: Hand Auger

Drilling Fluid: None

Borehole Diam: 3 inch

Contractor: USGS - Ohio District

$== \pm=-1$

=ニ二-

$==1$

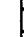

E=E-

E=E二-

$E==-1$

Begin Drilling: $12 / 5 / 96$

End Drilling:

$12 / 5 / 96$

Borehole TD: 3.1

Logged By: G. Nalley/J. Parnell

Orig Borehole \#: USB-180A

Converted to Well? NO
USGS Well Name:

Northing: 725157.81

Easting: 1886068.65

Surface Elev: 800

Datum: $f t, N G V D$

Air Force Plant 85 


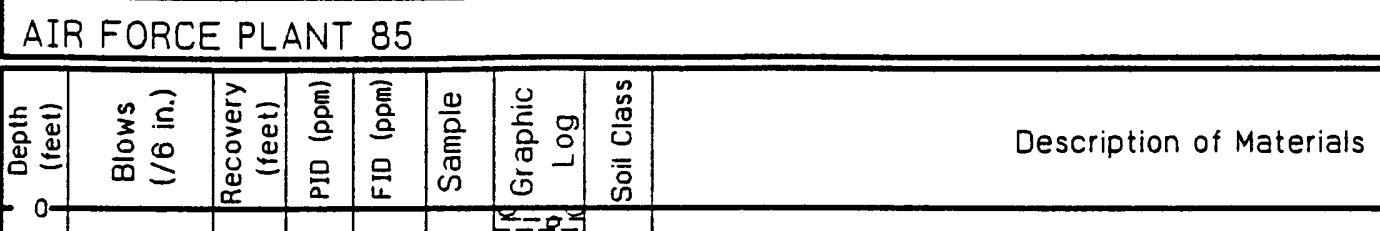

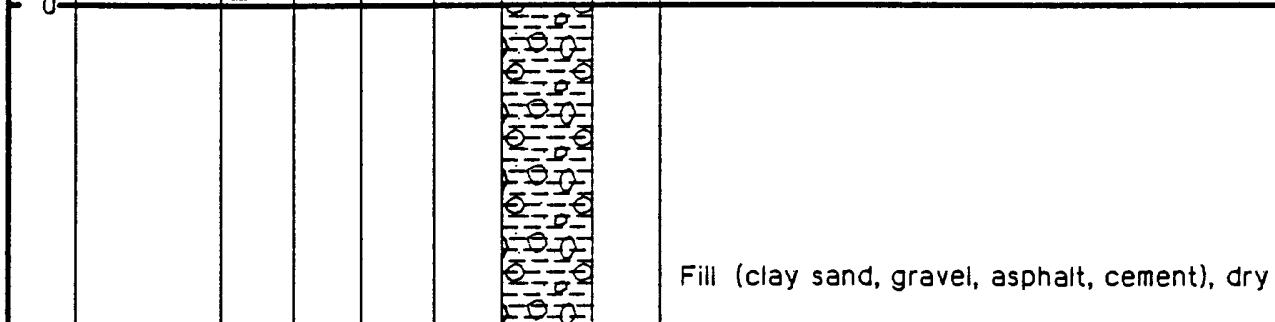

Drilling Co: U.S.G.S Rocky Mountain

Driller: Bill Gilman

Drilling Method: Hollow Stem Auger

Drilling Fluid: City of Columbus Water

Borehole Diam: 8.5 inch

Contractor: USGS - Ohio District
Begin Drilling: 12/5/96

End Drilling: $12 / 5 / 96$

Borehole TD: 2.3

Logged By: G. Nalley/J. Parnell

Orig Borehole \#: USB-180B

Converted to Well? NO
USGS Well Name:

Northing: $\quad 725248.42$

Easting: 1886131.37

Surface Elev: 800

Datum: $\mathrm{ft}, \mathrm{NGVD}$

Air Force Plant 85 


AIR FORCE PLANT $85 \quad$ Columbus, Ohio

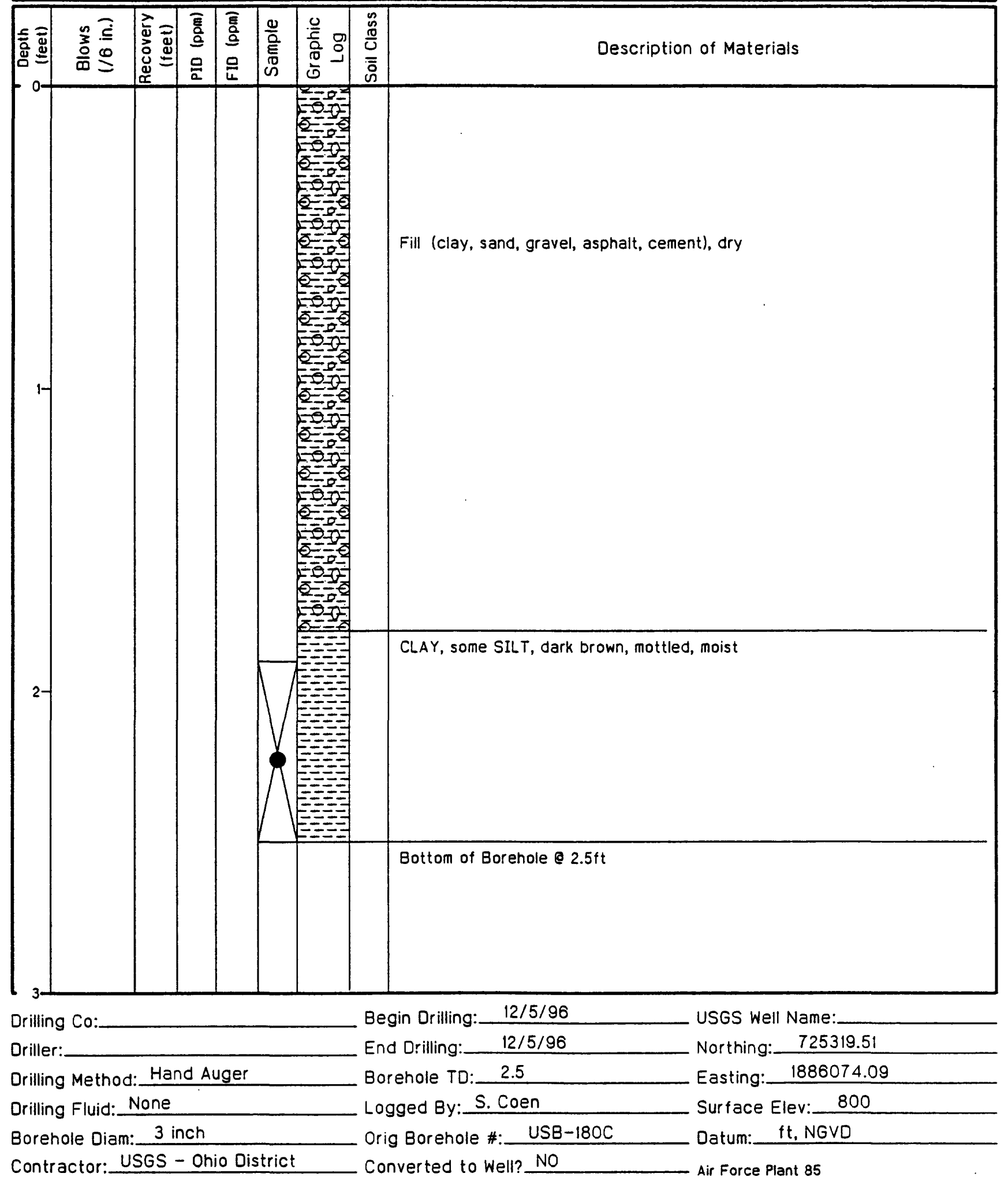




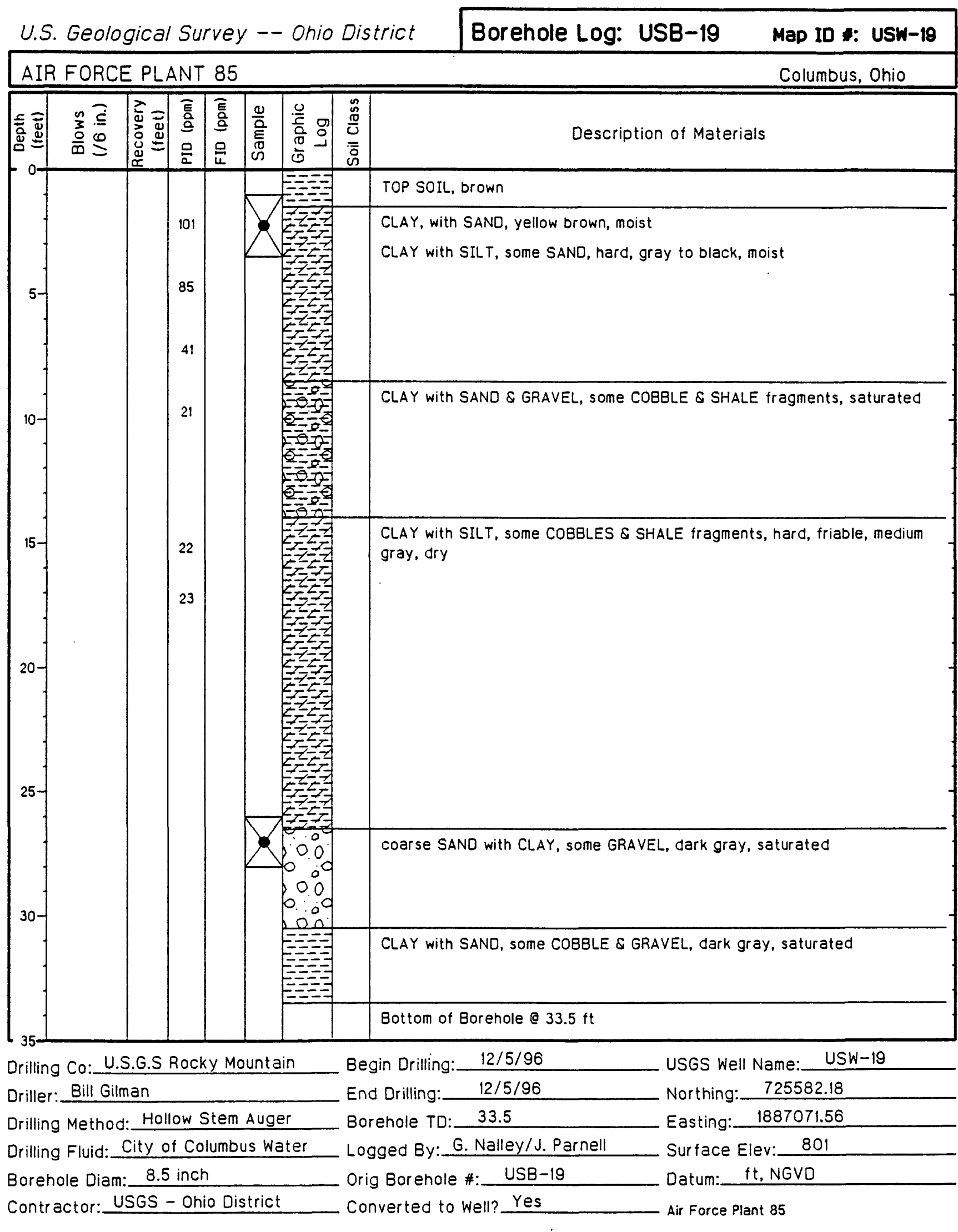




AIR FORCE PLANT $85 \quad$ Columbus, Ohio

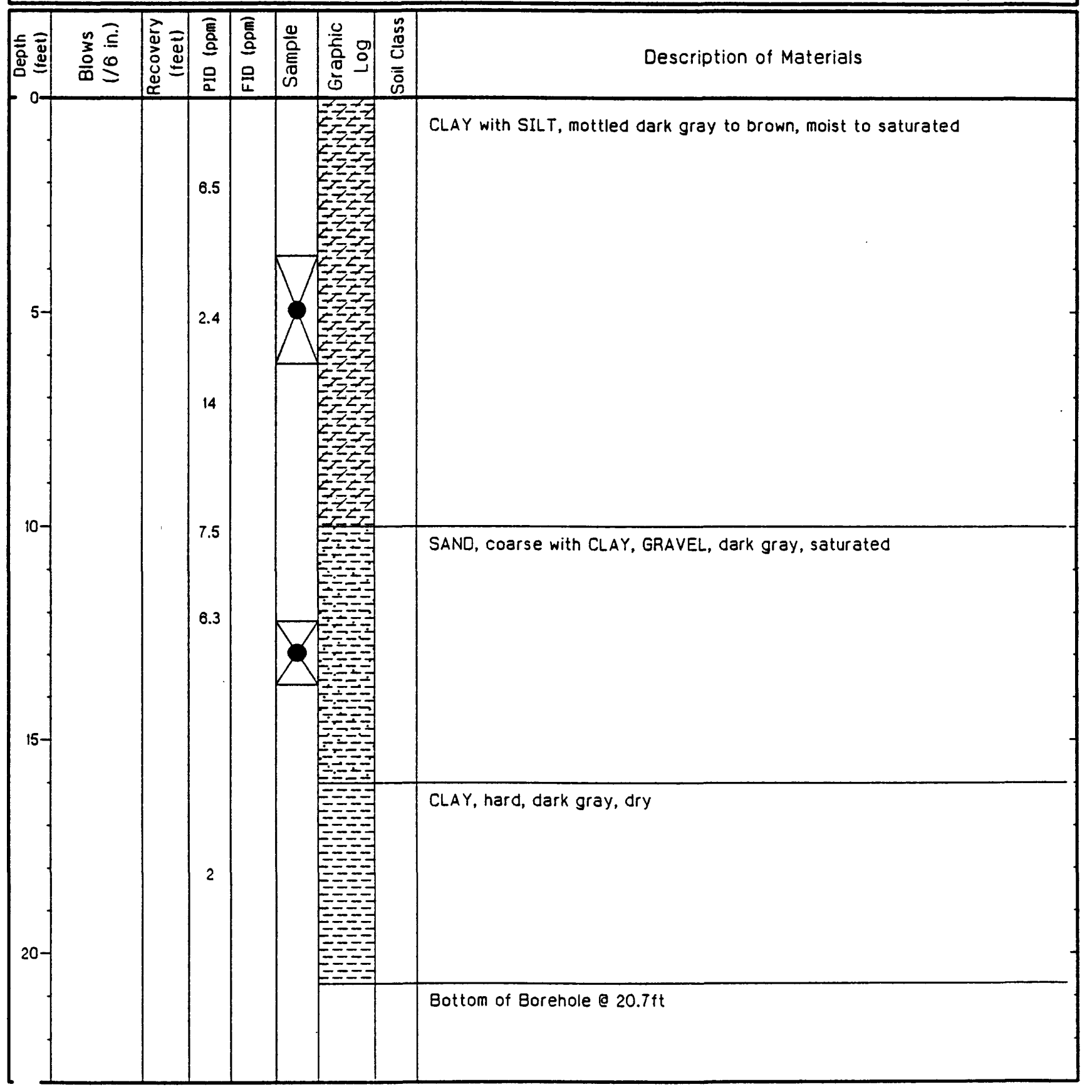

\begin{tabular}{|c|c|c|}
\hline Drilling Co: U.S.G.S Rocky Mountain & Begin Drilling: $12 / 6 / 96$ & USGS Well Name: \\
\hline Driller: Bill Gilman & End Drilling:__ & Northing: $\quad 724719.55$ \\
\hline Drilling Method: Hollow Stem Auger & Borehole TD: 20.7 & Easting: 1887866.32 \\
\hline Drilling Fluid: City of Columbus Water & Logged By: G. Nalley/J. Parnell & Surface Elev: 801 \\
\hline Borehole Diam: 8.5 inch & Orig Borehole \#: USB-20 & Datum: $\mathrm{ft}, \mathrm{NGVD}$ \\
\hline Contractor: USGS - Ohio District & Converted to Well? NO & Air Force Plant 85 \\
\hline
\end{tabular}


U.S. Geological Survey -- Ohio District Borehole Log: USB-21

Map ID : : USB-21

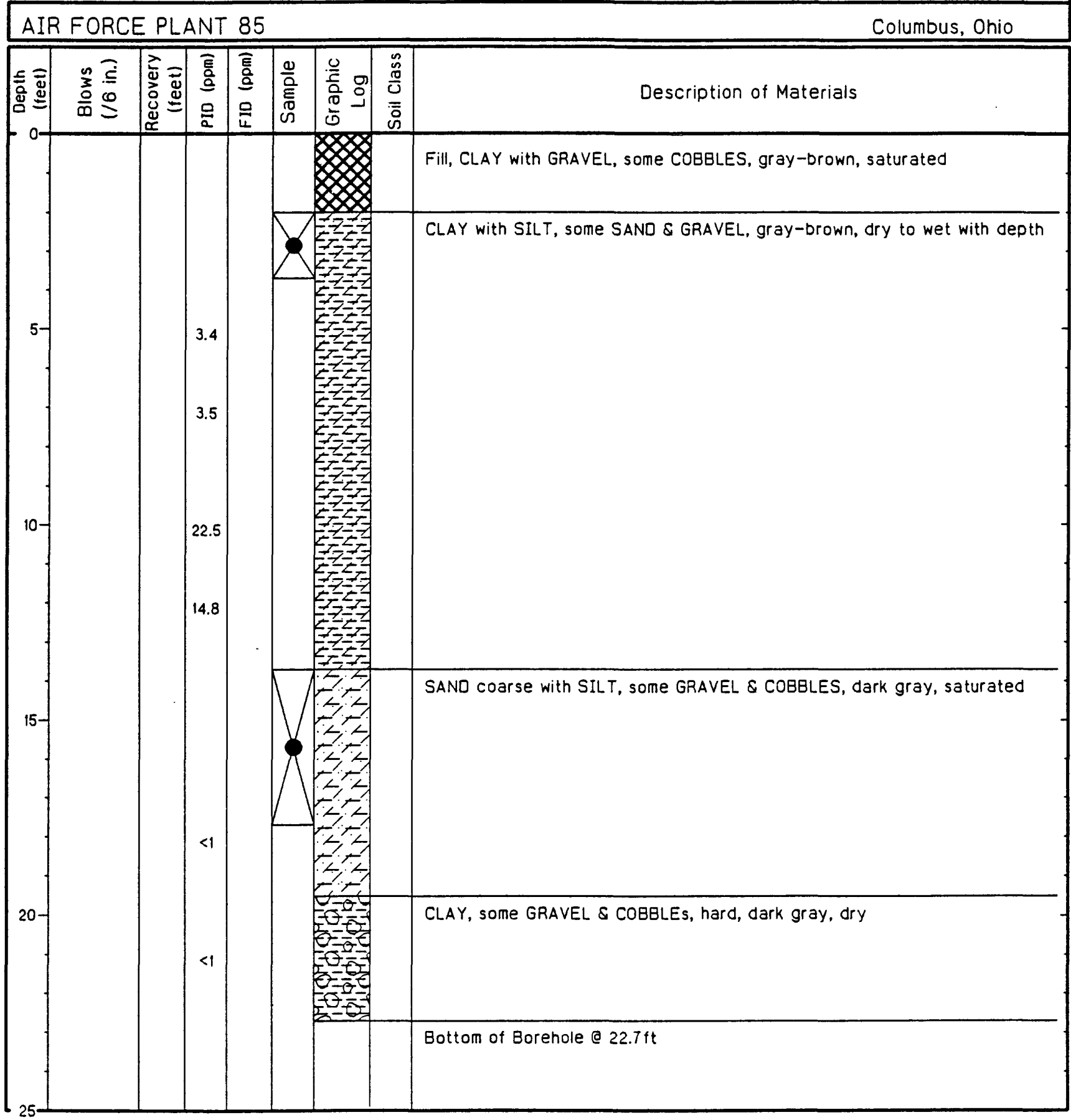

Drilling Co:U.S.G.S Rocky Mountain

Driller:Bill Gilman

Begin Drilling: 12/7/96

USGS Well Name:

Drilling Method: Hollow Stem Auger End Drilling: $12 / 7 / 96$ Northing: $\quad 725591.58$

Drilling Fluid: City of Columbus Water Borehole TD: 22.7 Easting: 1887914.55

Borehole Diam: 8.5 inch Logged By: G. Nalley/J. Parnell Surface Elev: 802

Contractor: USGS - Ohio District Orig Borehole \#: USB-21 Converted to Well? NO Datum: $\mathrm{ft}, \mathrm{NGVD}$ Air Force Plant 85 
APPENDIX 2. CONSTRUCTION DIAGRAMS FOR OBSERVATION WELLS AT AIR FORCE PLANT 85, COLUMBUS, OHIO, 1996 


\begin{tabular}{|c|c|c|}
\hline Drilling Co: U.S.G.S Rocky Mountain & $11 / 16 / 96$ & d By: G. Nalley/J. Parnell \\
\hline Driller:Bill Gilman & $11 / 17 / 96$ & Borehole Diam: 8.5 inch \\
\hline Drilling Method: Hollow Stem Auger & all: $11 / 17 / 96$ & Northing: $\quad 725067.47$ \\
\hline Drilling Fluid: City of Columbus Water & $11 / 17 / 96$ & 1890680.09 \\
\hline
\end{tabular}

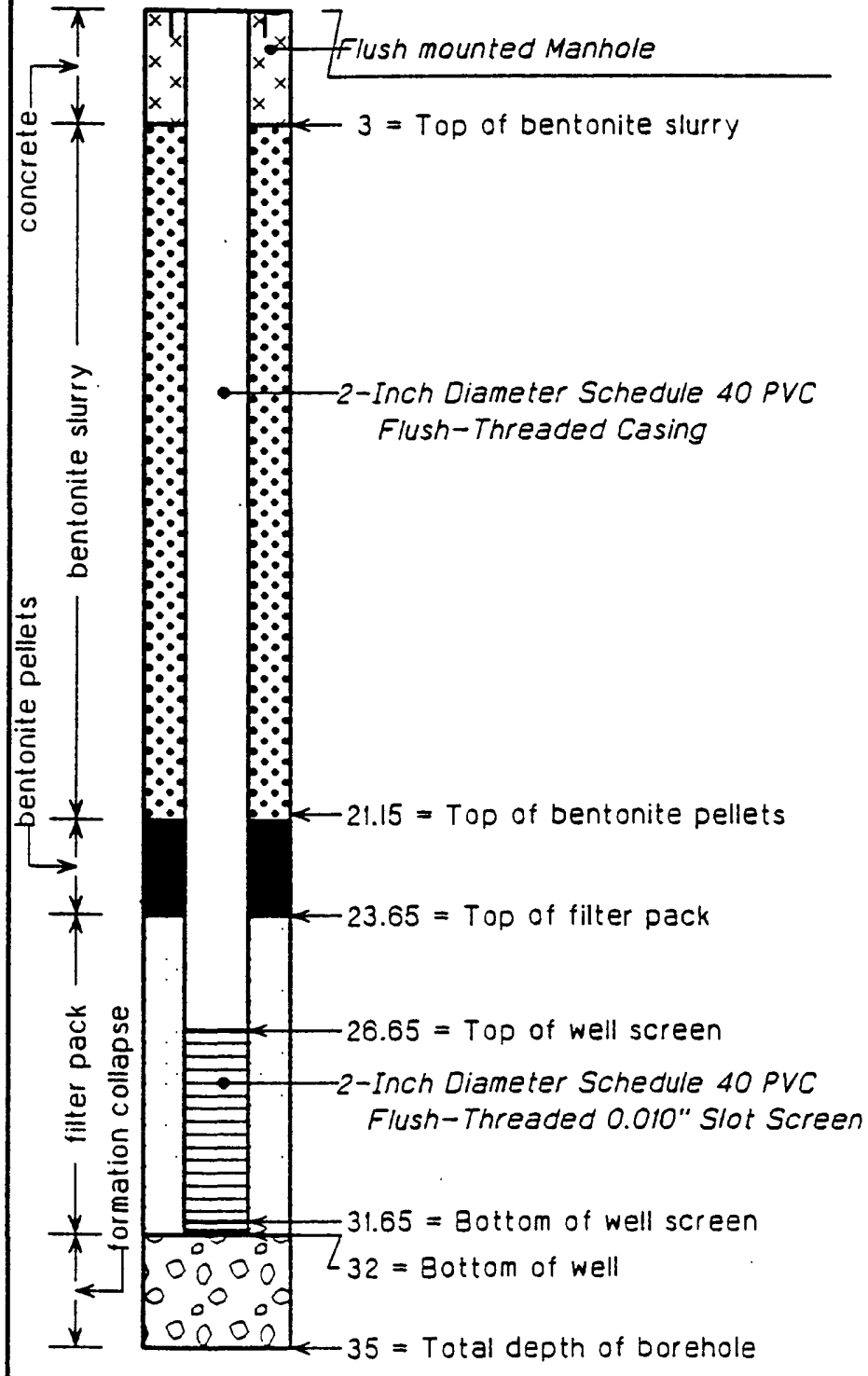

Map ID \#: USWO2

Amount Used: 8 bags

Seal Type: Bentonite Pellets

Hydration Time: 48 hours

Emplacement Method:_. Pour

Grout Comp/Prop: Cement/bentonite

Emplacement Method:_Pour

Centralizer Depth (s): None

Protective Casing Type: Steel

Diameter: 8-inch

Date Installed: $\quad 11 / 18 / 96$

Weep Hole?: no

Number of Guard Posts: None

OTW (bmp) upon completion:

Date:

Development Technique: Bail

Begin Development: 12/16/96

End Development: $\quad 12 / 17 / 96$

Vol Fluid Removed: 26 gallons

Measuring Pt Elev:

Ground Surface Elev: 806

Measuring Pt Desc: Top of PVC

Datum: $f t$, NGVO

Surveyed By:

IRP Well \#:

Contractor: USGS - Ohio District

Remarks: 


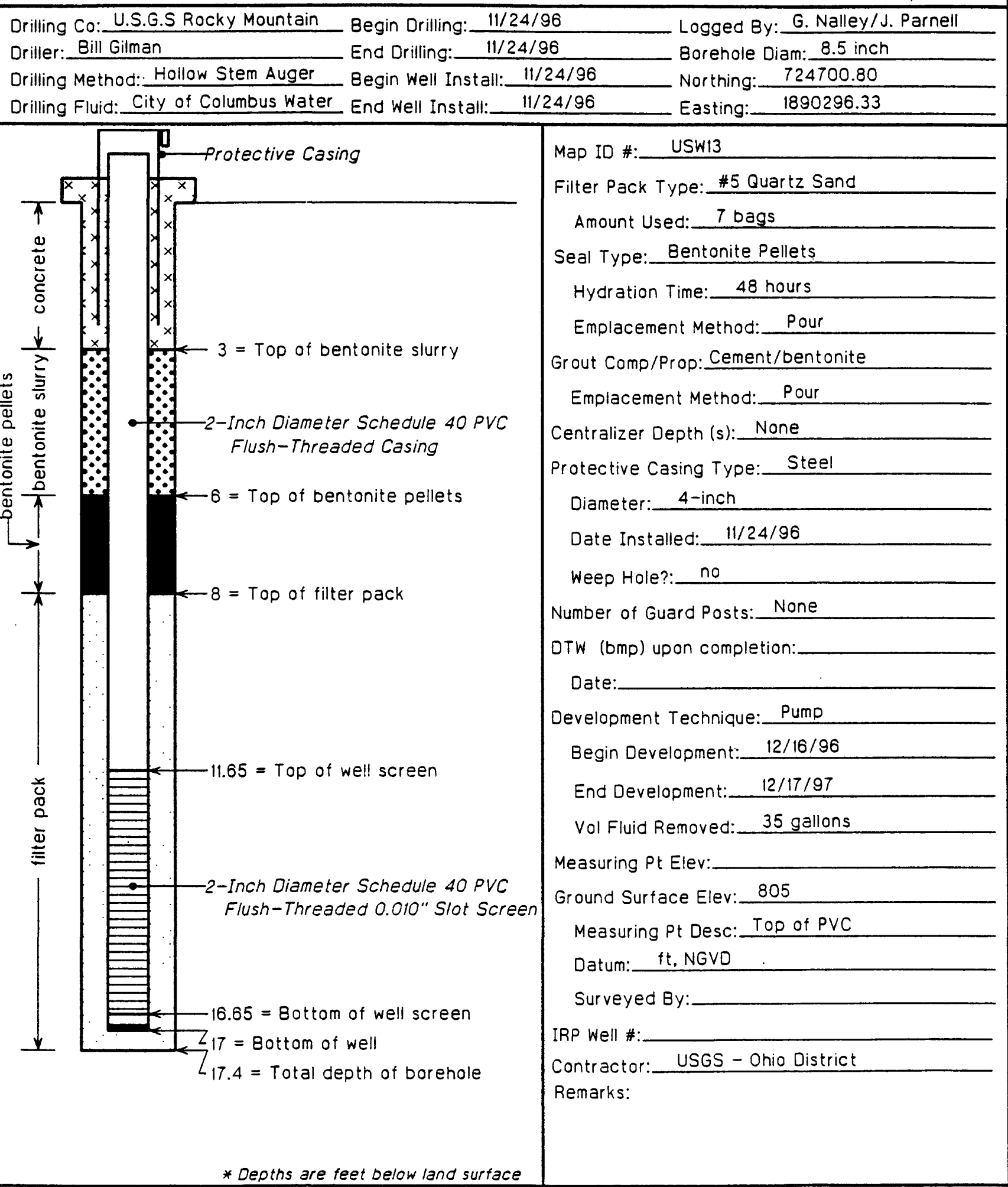

For Lithologic Descriptions See Log of Borehole: USB13 


\begin{tabular}{|c|c|c|}
\hline Drilling Co: U.S.G.S Rocky Mountain & $12 / 04 / 96$ & G. Nalley/J. Parnell \\
\hline Oriller: Bill Gilman & $12 / 04 / 96$ & Borehole Diam: 8.5 inch \\
\hline Drilling Method: Hollow Stem Auger & all: $12 / 04 / 96$ & Northing: $\quad 725350.85$ \\
\hline Drilling Fluid: City of Columbus Water & $12 / 04 / 96$ & 1886531.12 \\
\hline
\end{tabular}

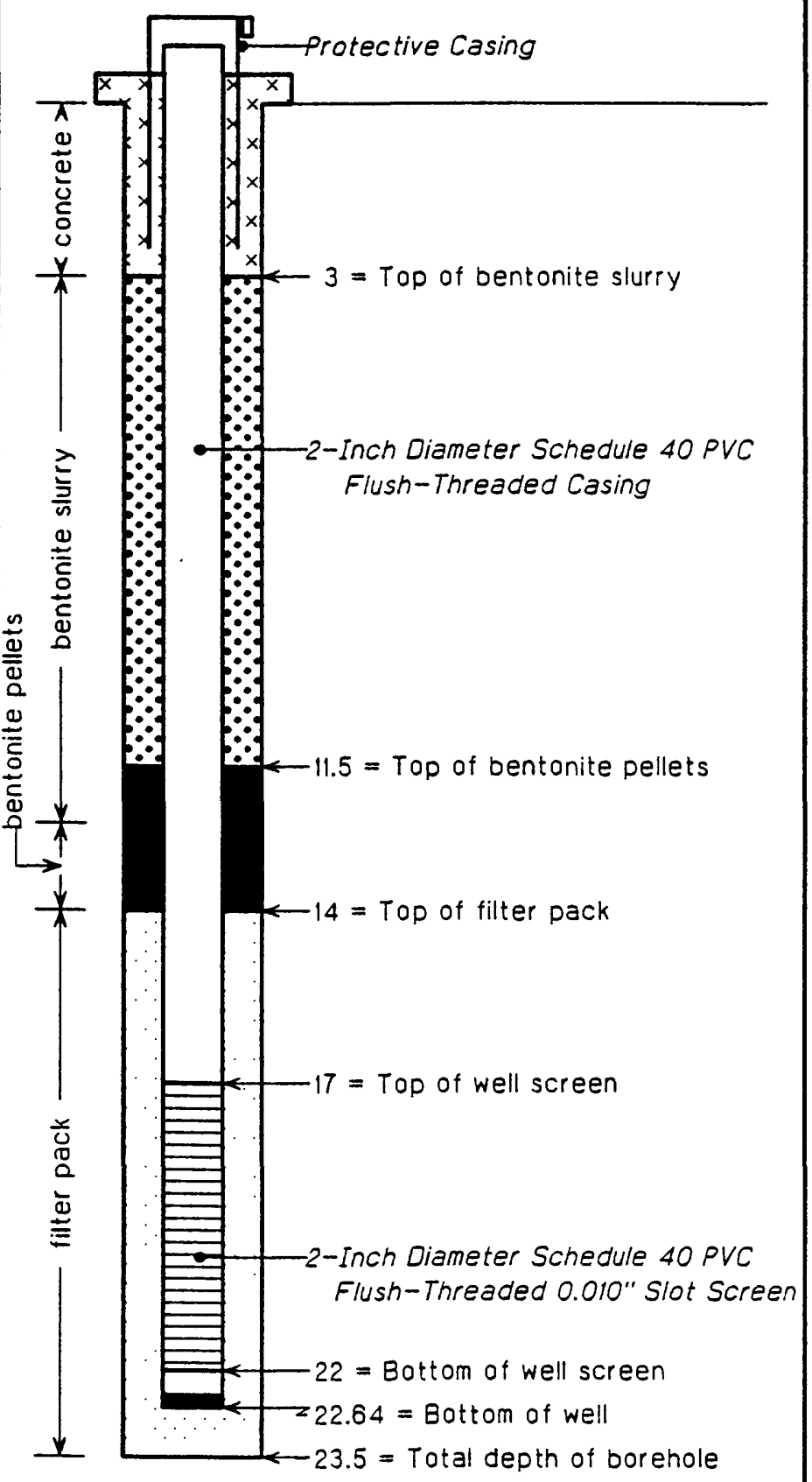

* Depths are feet Delow land surface

Map ID \#:_ USW16

Filter Pack Type:\#5 Quartz Sand

Amount Used: 6 bags

Seal Type: Bentonite Pellets

Hydration Time: 48 hours

Emplacement Method:_Pour

Grout Comp/Prop: Cement bentonite

Emplacement Method:_Pour

Centralizer Depth (s): None

Protective Casing Type: Steel

Diameter: 4-inch

Date Installed: 12/04/96

Weep Hole?: no

Number of Guard Posts: None

DTW (bmp) upon completion:

Date:

Development Technique: Pump

Begin Development: 12/16/96

End Development:_ 12/17/96

Vol Fluid Removed: 60 gallons

Measuring Pt Elev:

Ground Surface Elev: 801

Measuring Pt Desc: ToP of PVC

Datum: ft, NGVD

Surveyed By:

IRP Well \#:

Contractor:_USGS - Ohio District

Remarks: 


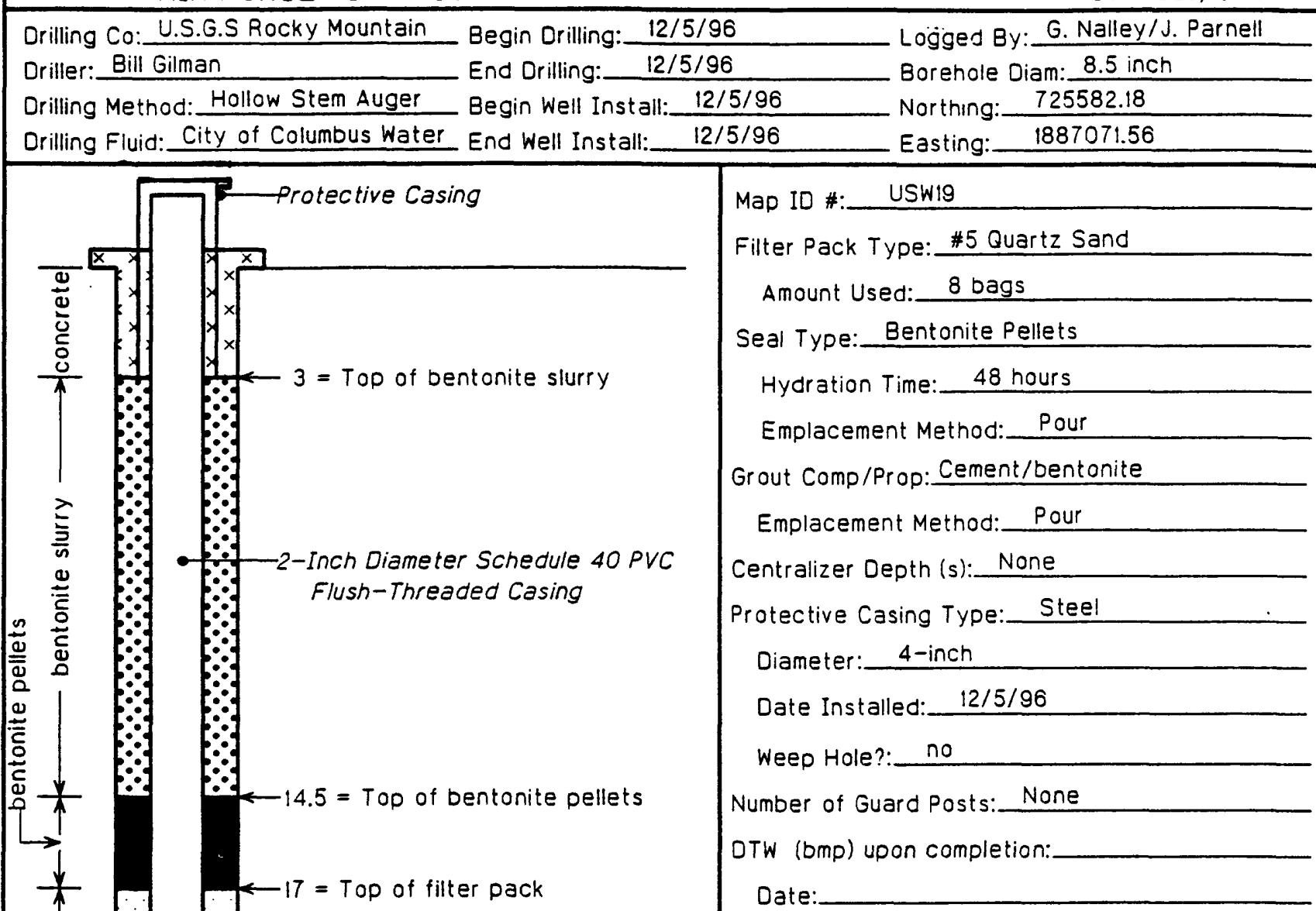

Date

Development Technique: Bail, Pump

Begin Development: 12/16/96

End Development: $\quad$ 12/17/96

Vol Fluid Removed: 50 gallons

Measuring Pt Elev:

Ground Surface Elev: 801

Measuring Pt Desc: Top of PVC

Datum: ft, NGVD

Surveyed By:

IRP Well \#: USWI9

Contractor: USGS - Ohio District

Remarks:

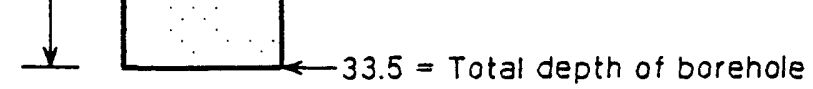

* Depths are feet Delow land surface

For Lithologic Descriptions See Log of Borehole: USB19 
APPENDIX 3. SUMMARY DATA - ANALYTICAL RESULTS FOR SOIL, GROUND WATER, SURFACE WATER, AND STREAMBED SEDIMENT AT AIR FORCE PLANT 85, COLUMBUS, OHIO, 1996 


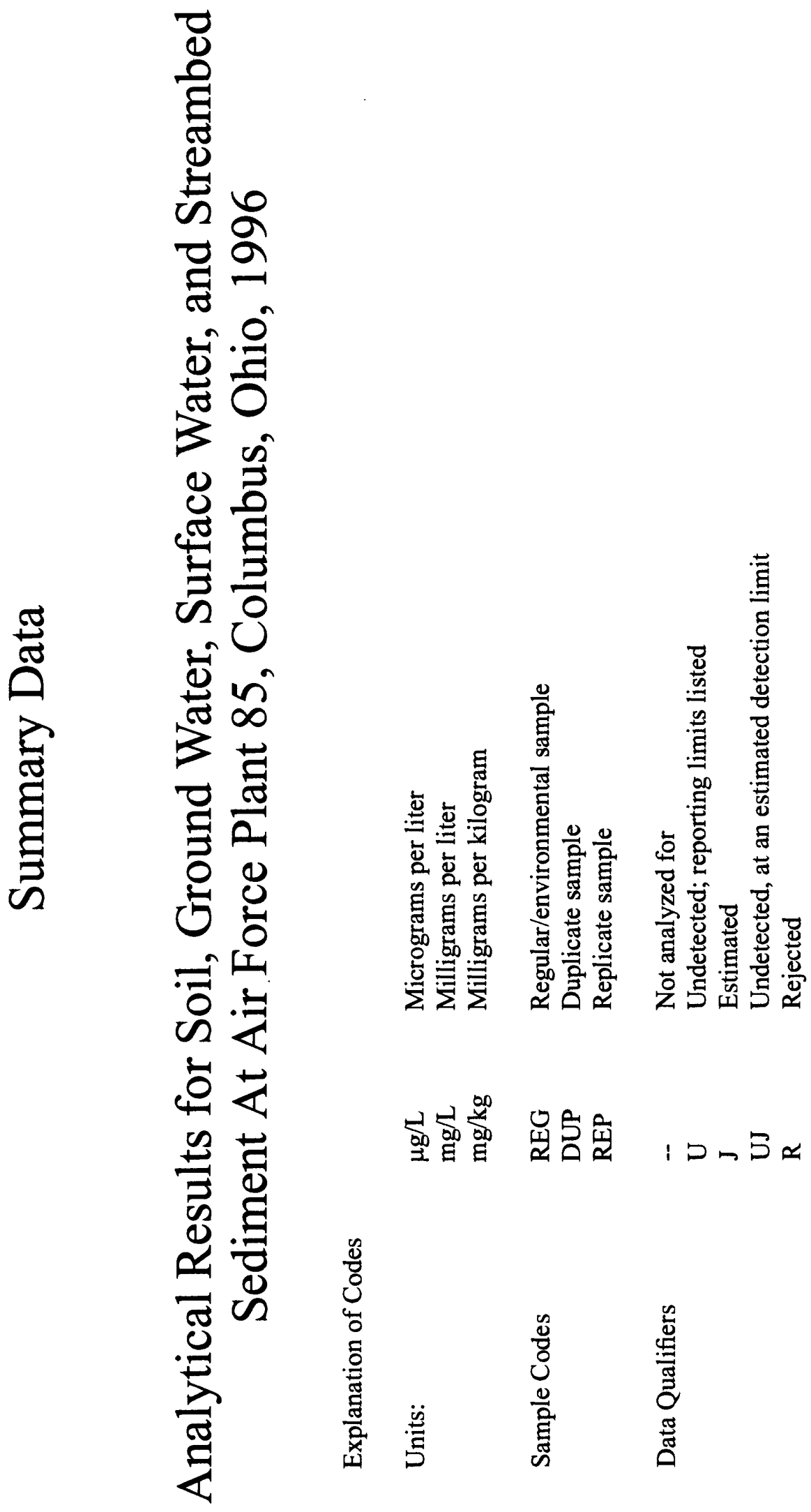




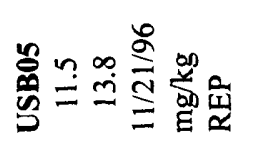

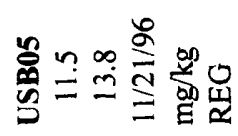

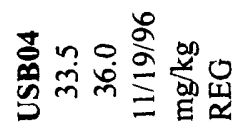

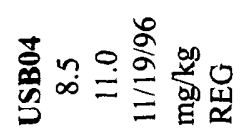

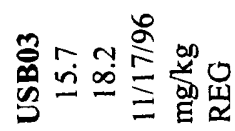

ڤे

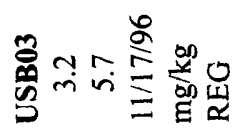

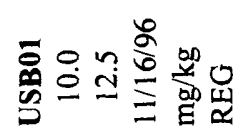

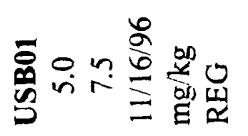

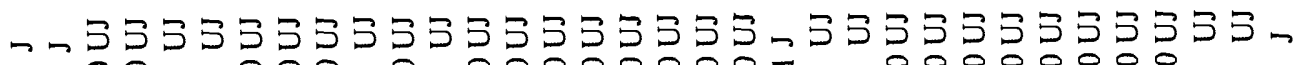

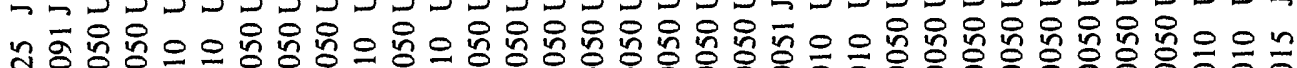

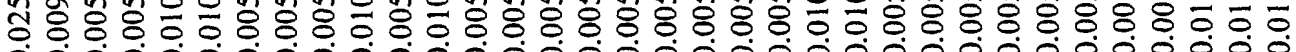

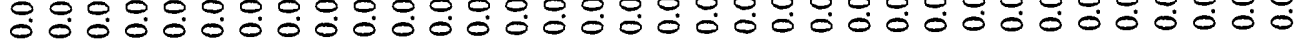

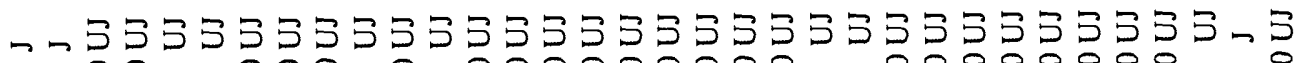

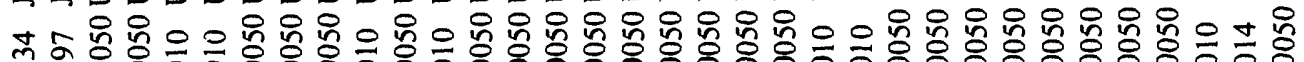

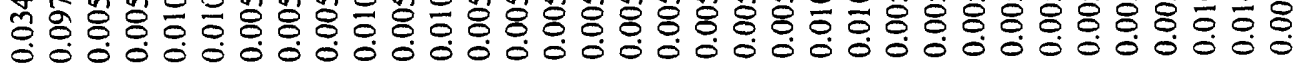

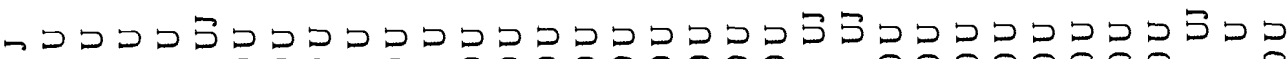

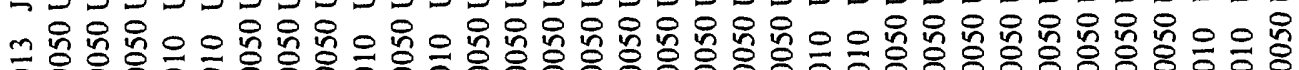

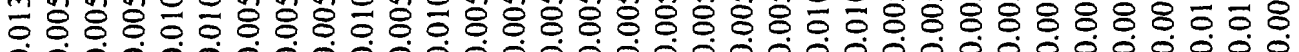
0000000000000000000000000000000000

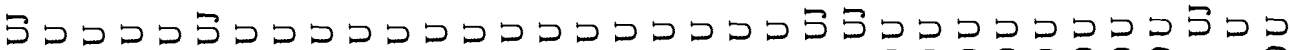

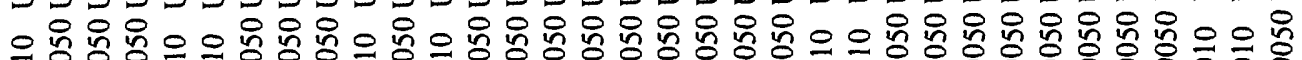
年0

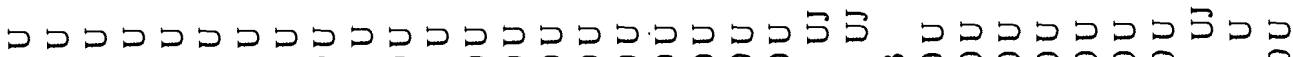

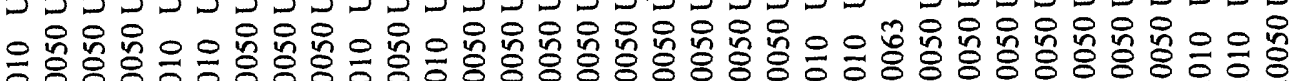

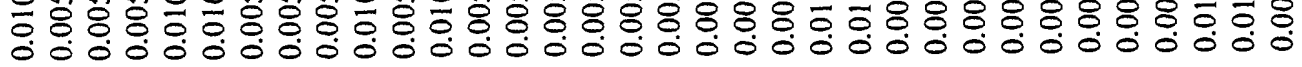

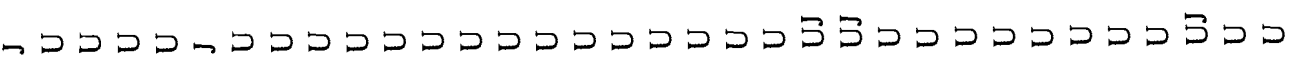

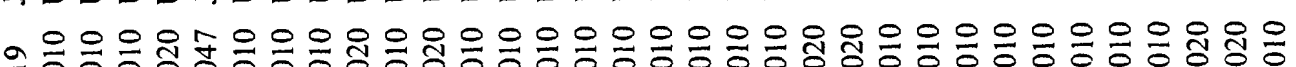

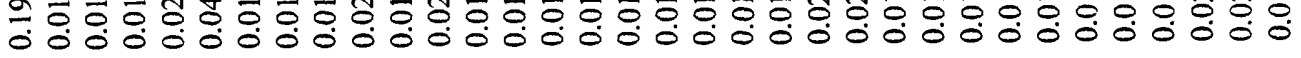

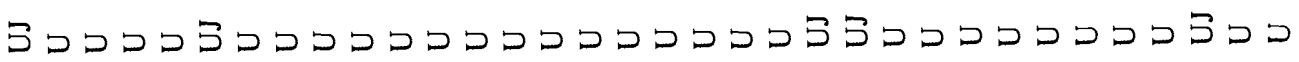

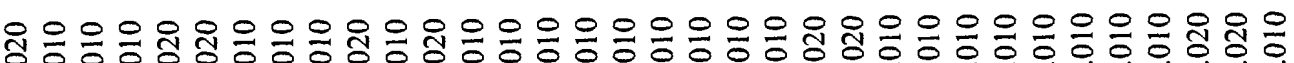

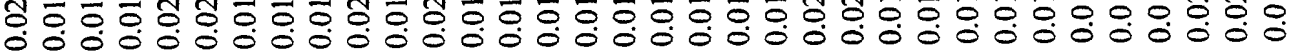

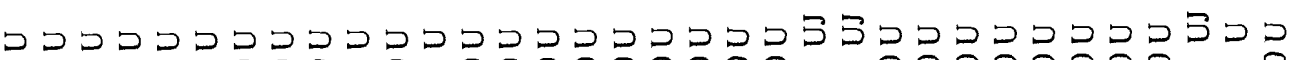

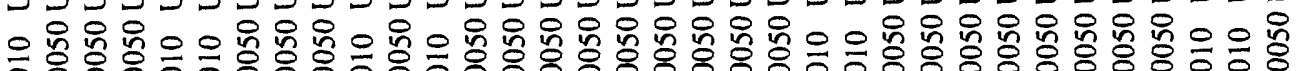


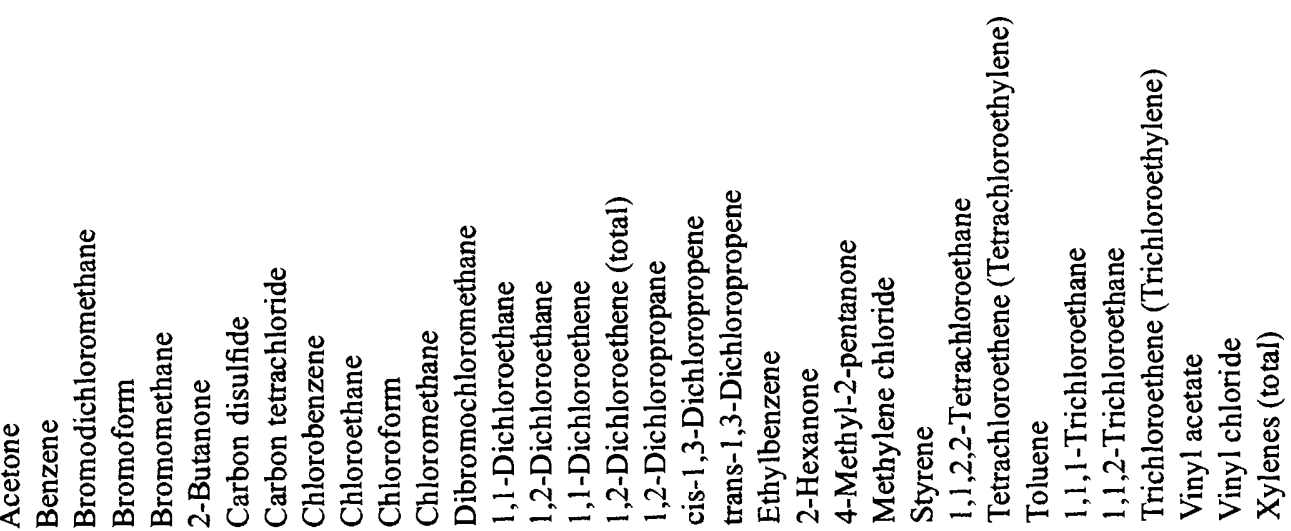




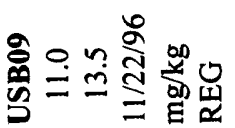

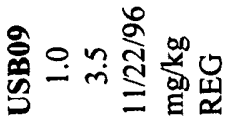

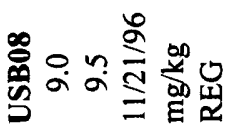

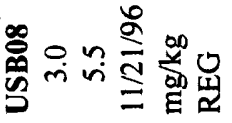

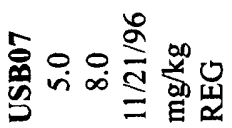

के

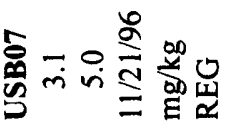

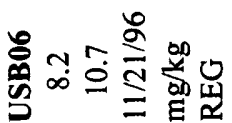

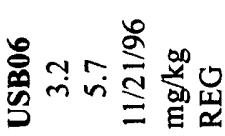

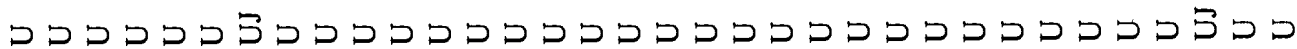

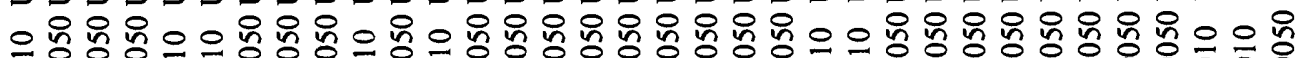

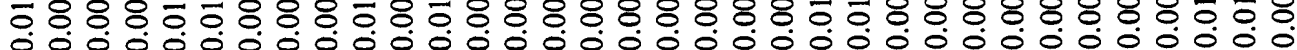

ט

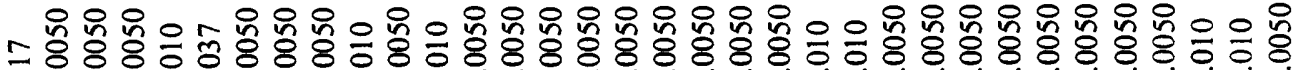

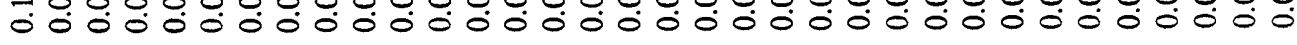

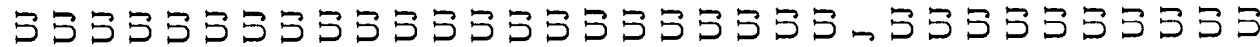

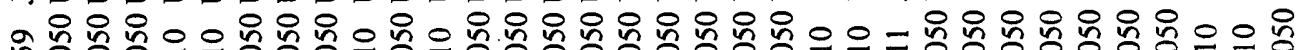

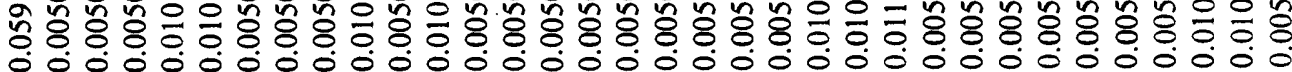

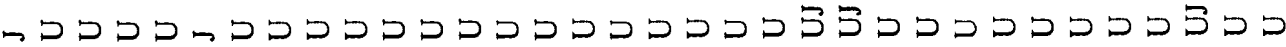

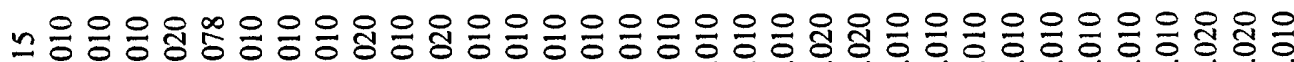

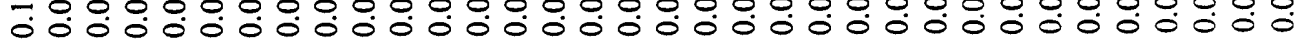

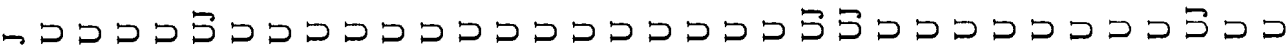

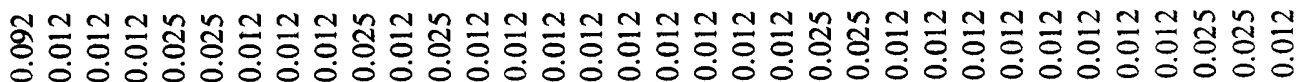

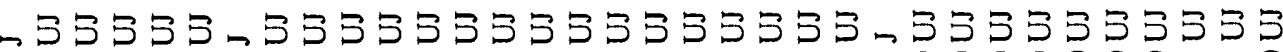

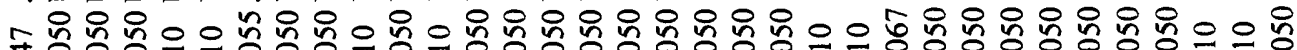

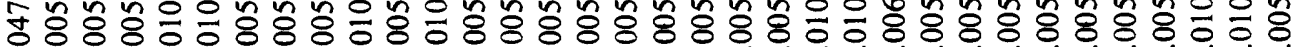

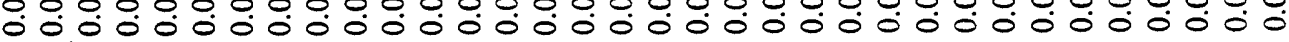

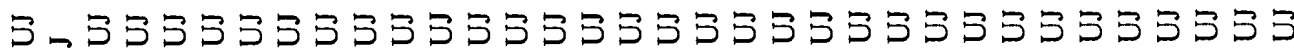

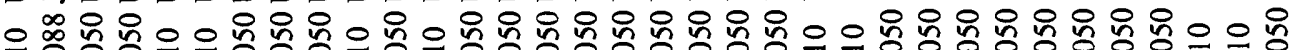

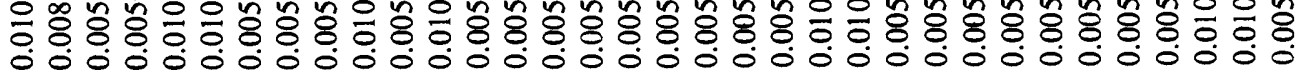

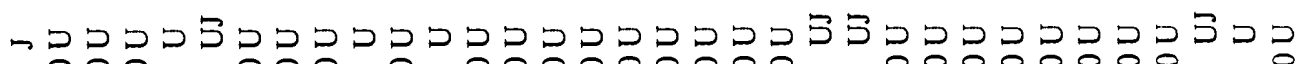

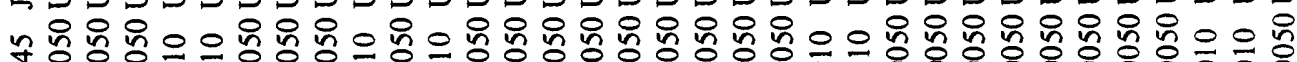

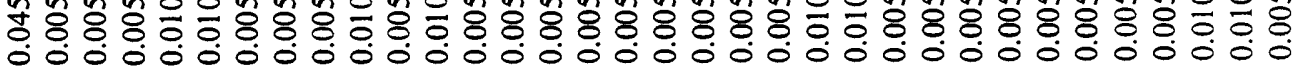
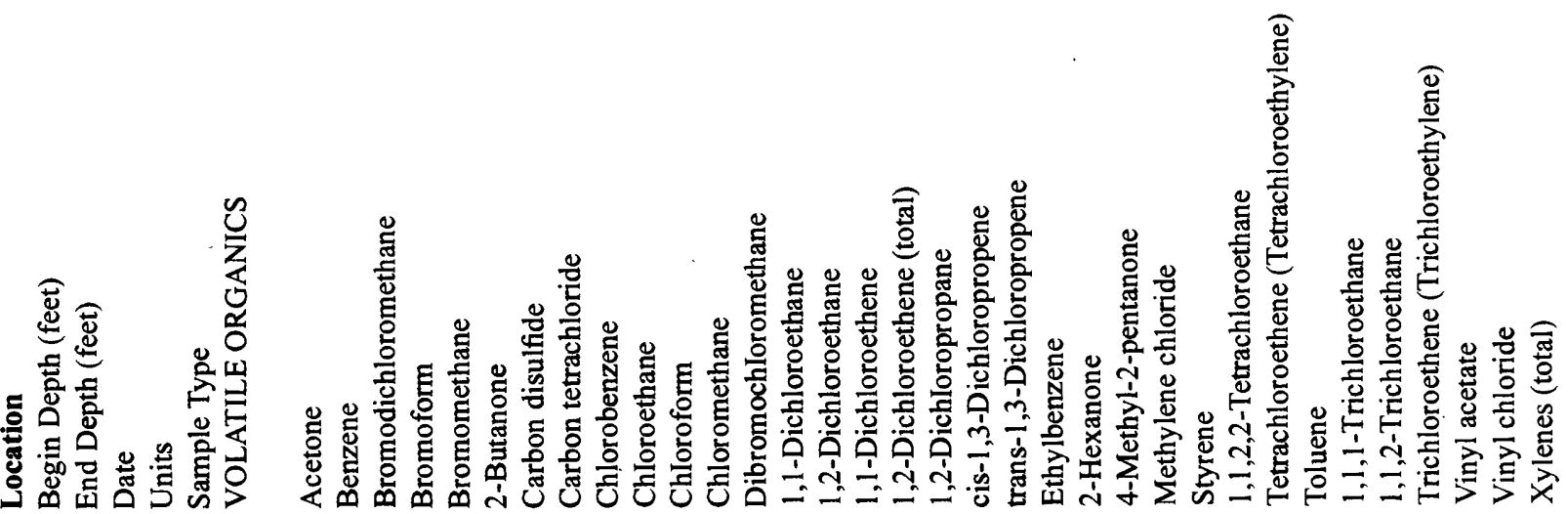


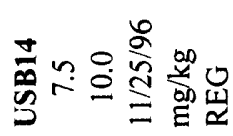

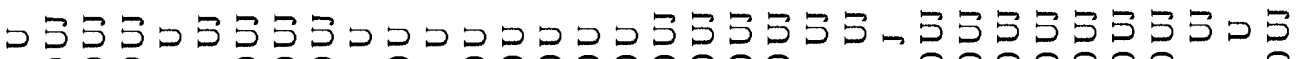

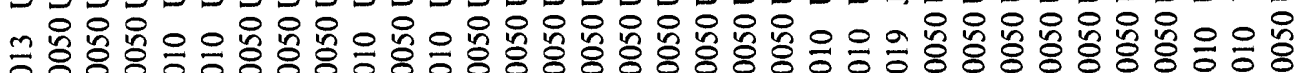

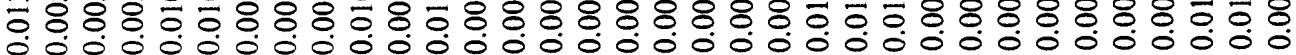

总

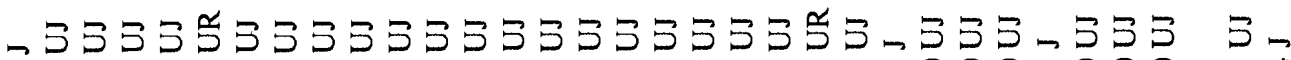

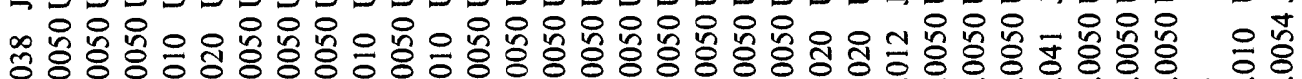

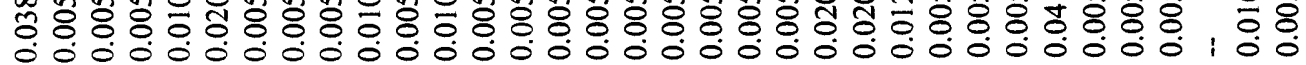

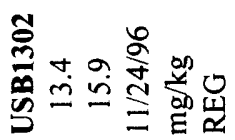

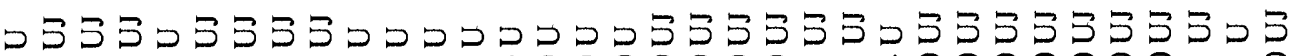

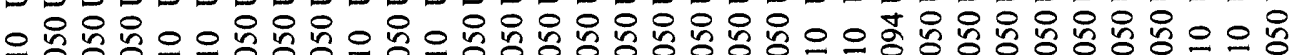

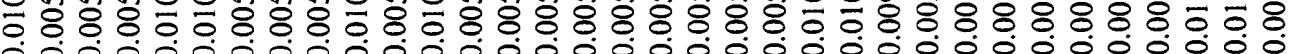

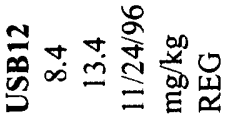

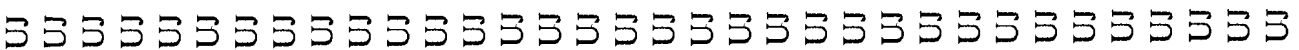

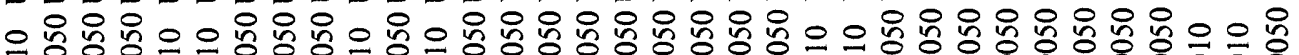

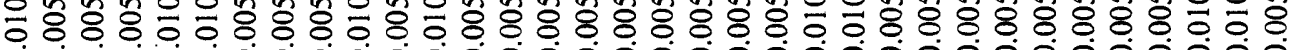

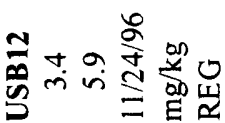

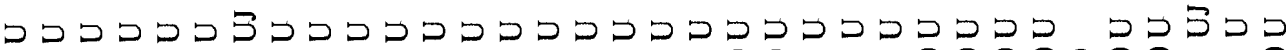

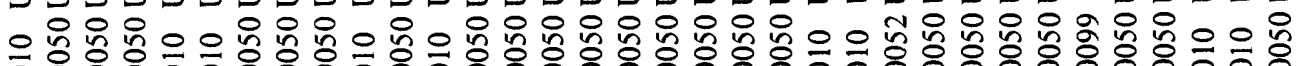
o.

\%

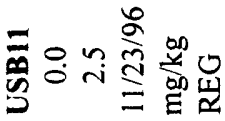

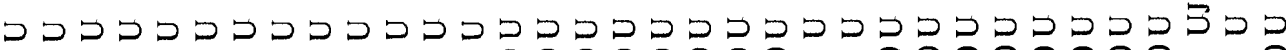

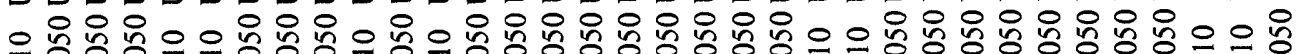

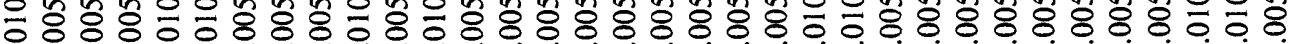

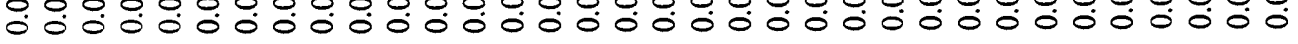

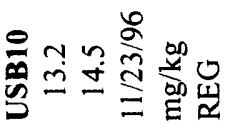

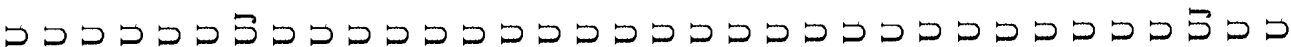

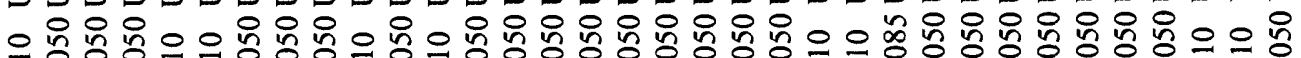

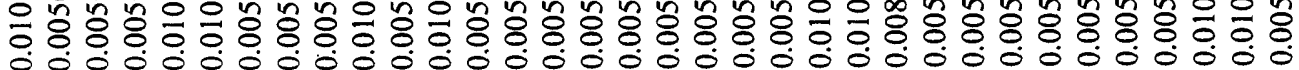

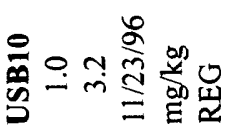

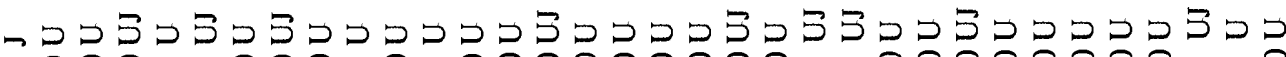
 \%

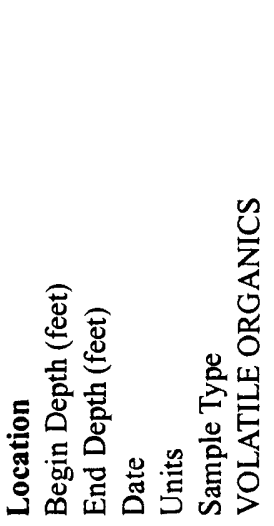

吾
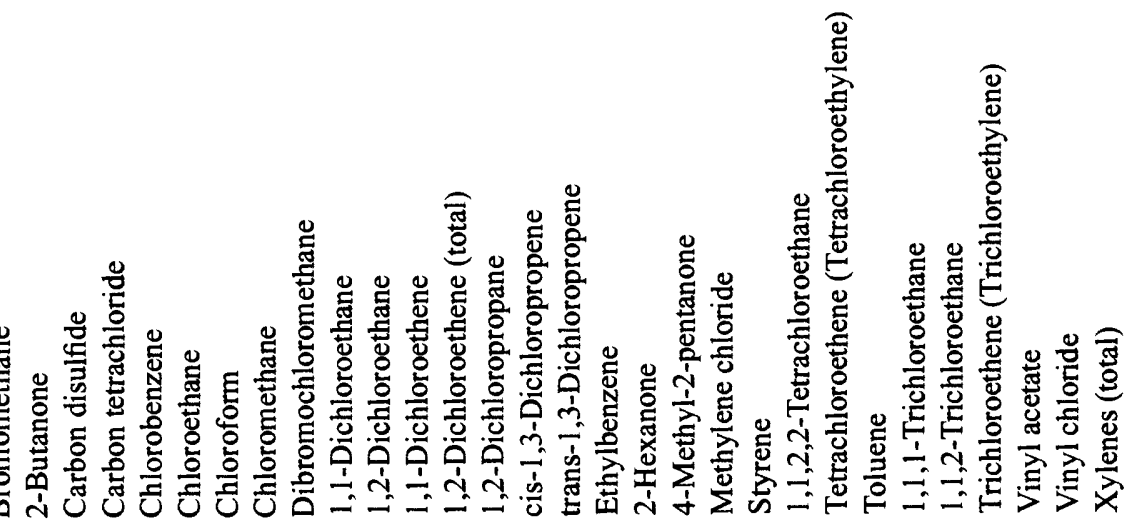


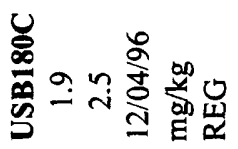

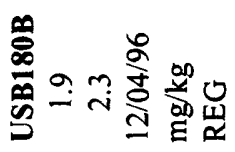

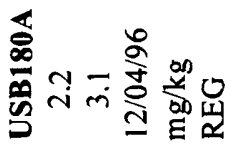

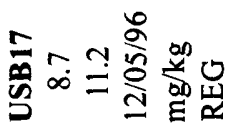

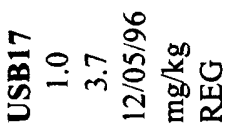

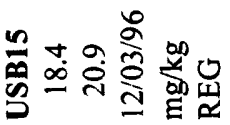

แ

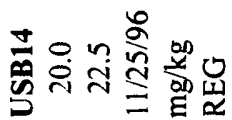

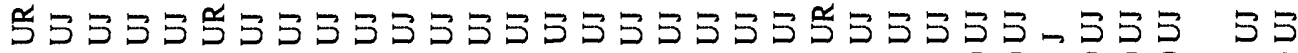

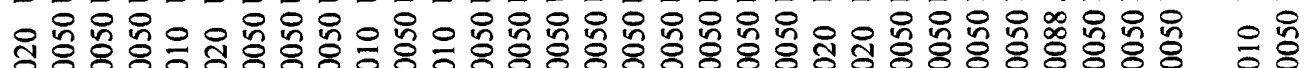

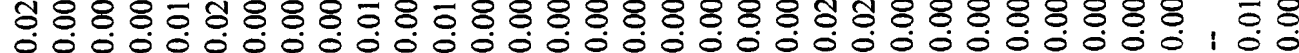

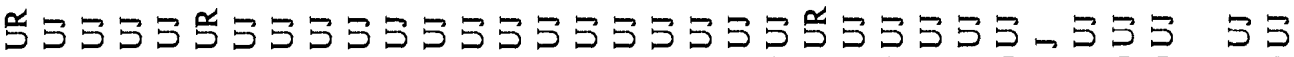

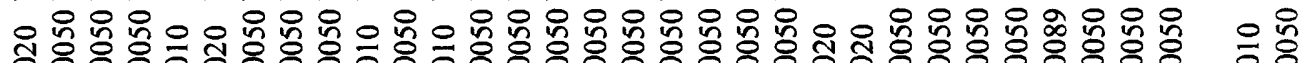

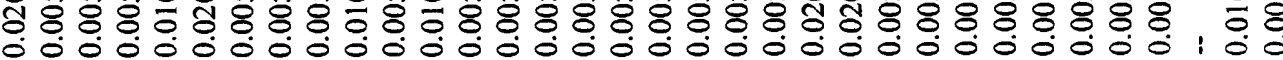

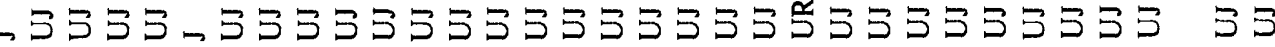

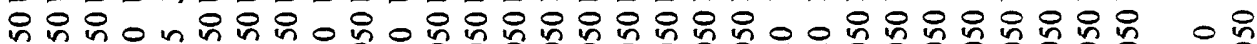

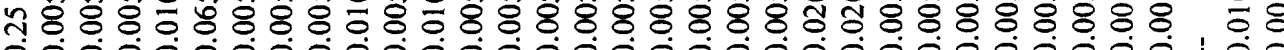
○

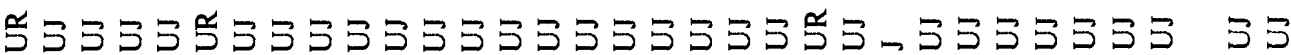

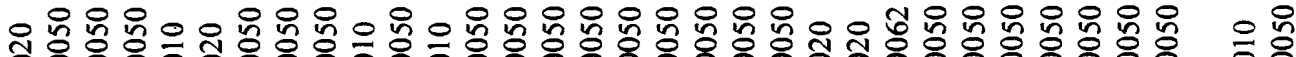

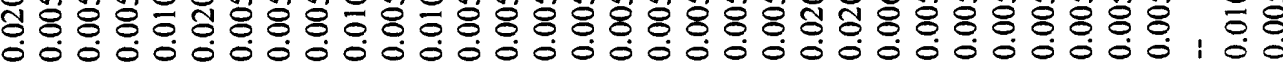

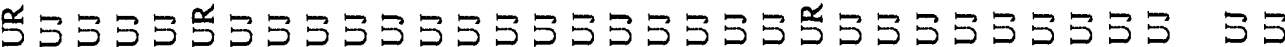

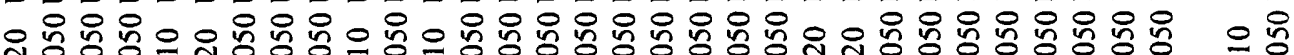

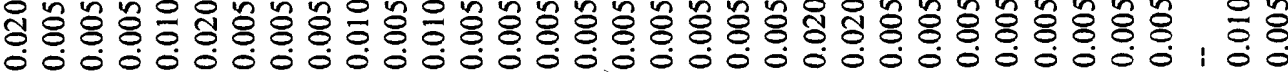

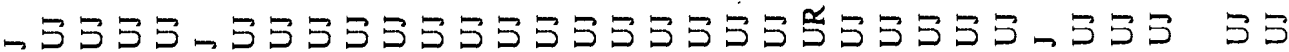

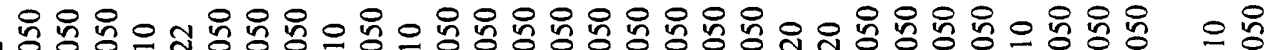

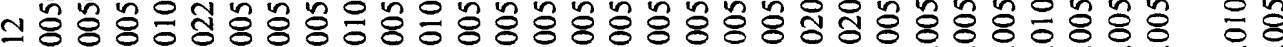

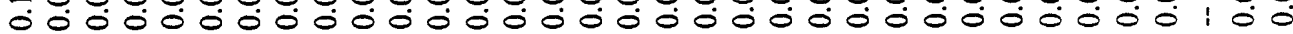

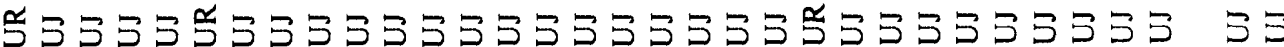

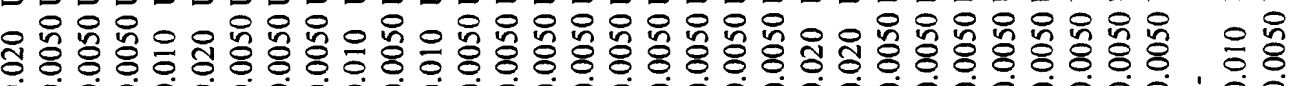

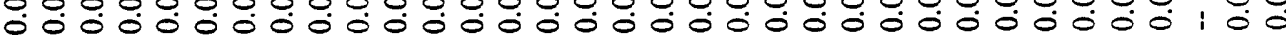

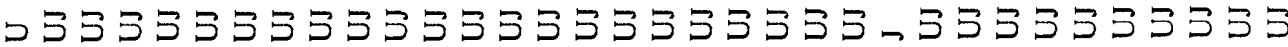

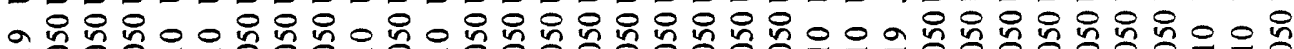

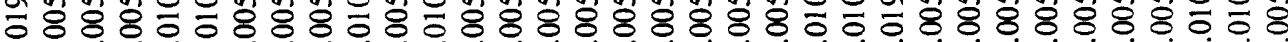
0000000000000000000000000000000000
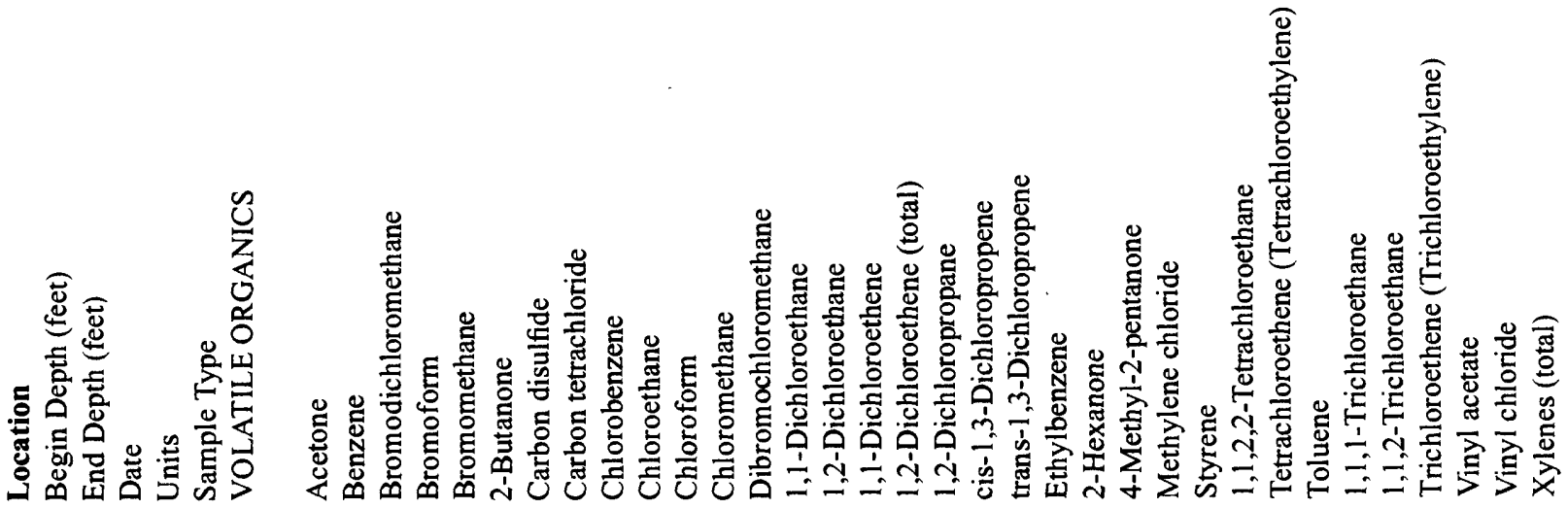


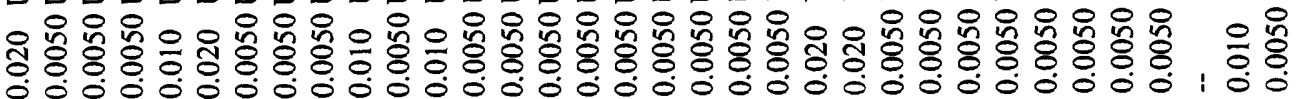

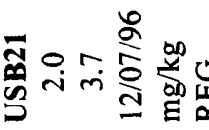

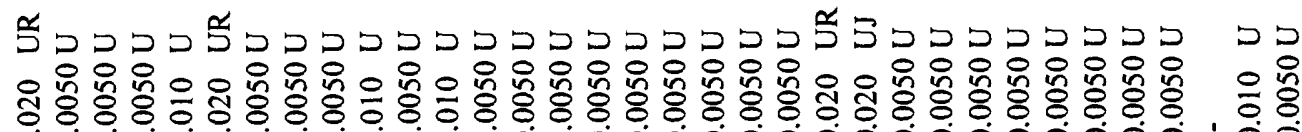

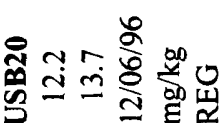

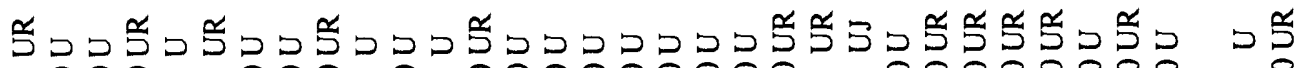

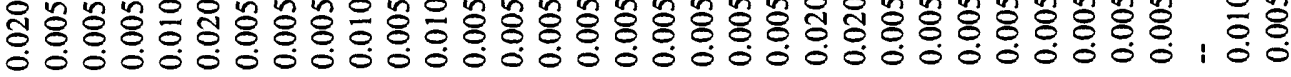

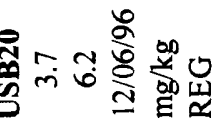

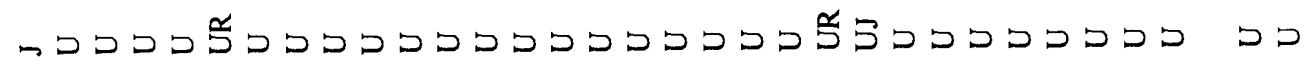

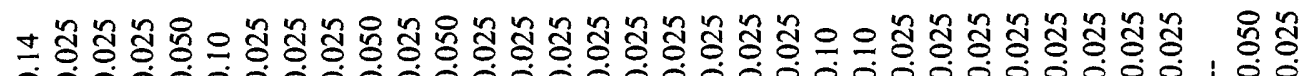

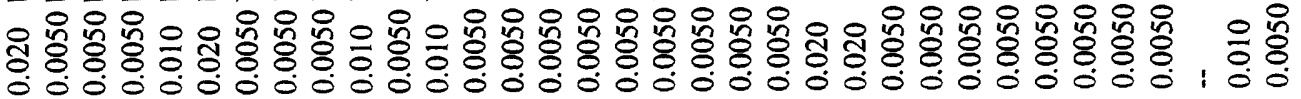$$
\text { . }
$$
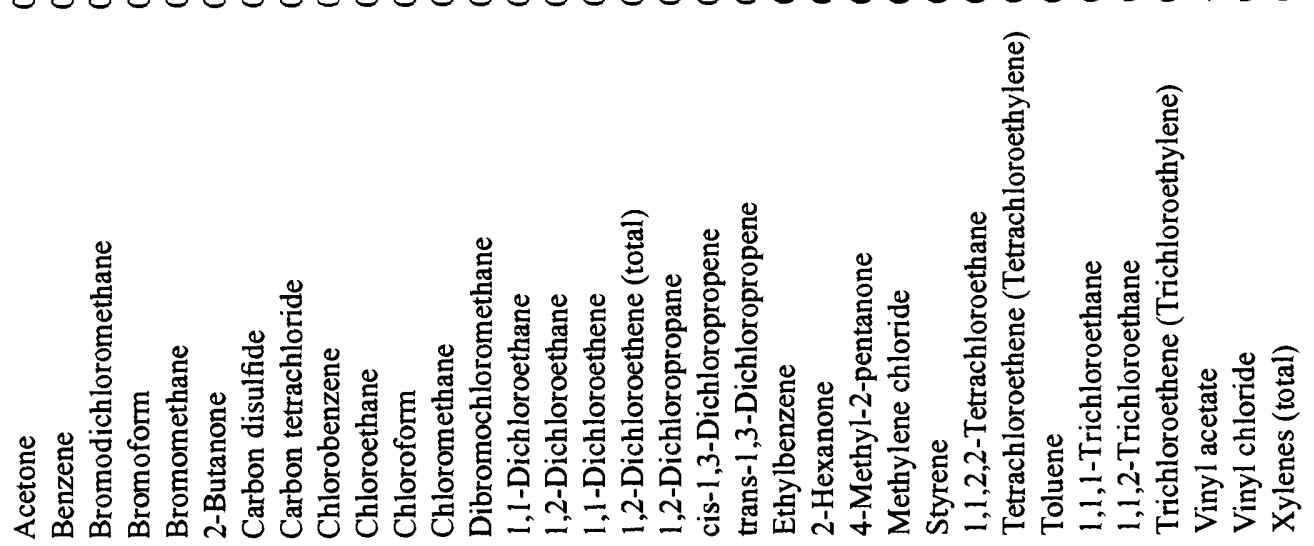


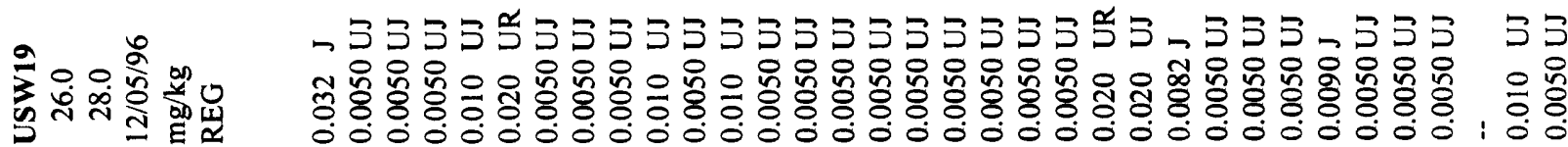

高

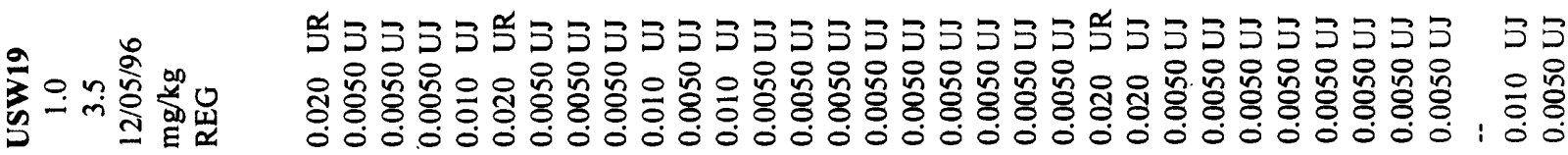

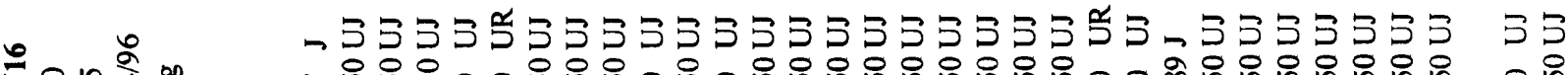

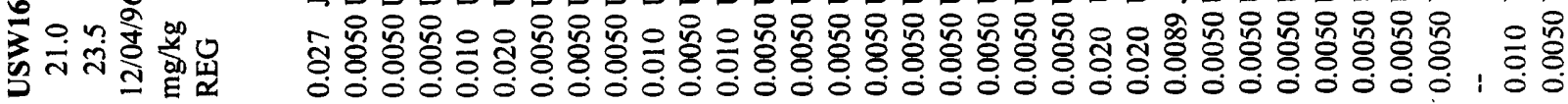

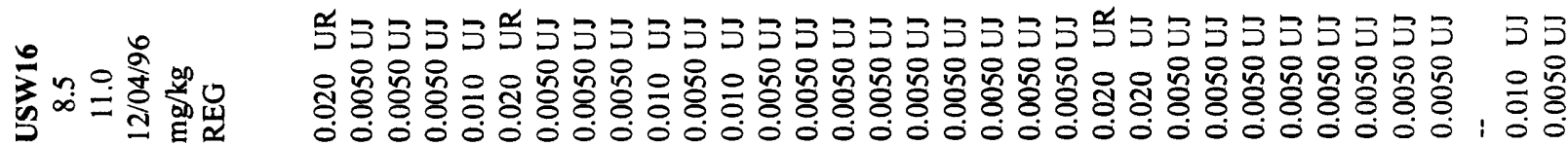

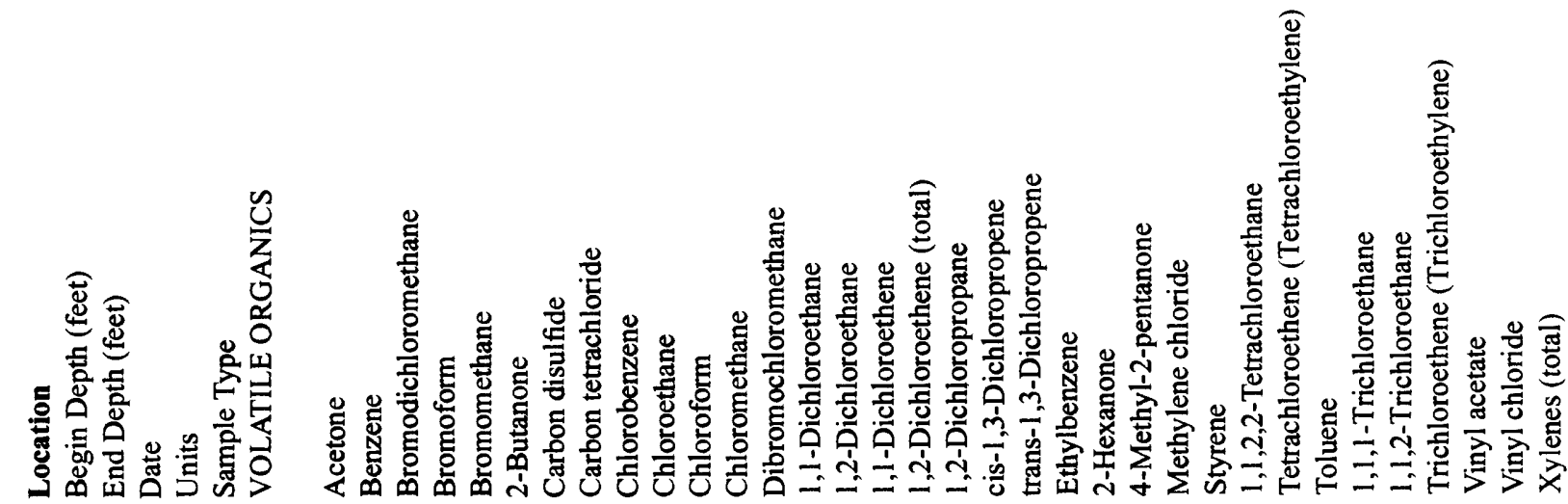




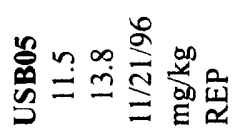

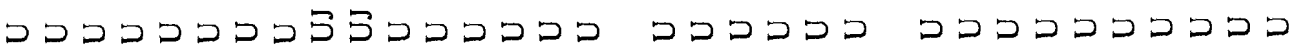

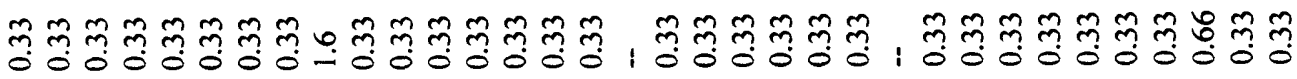

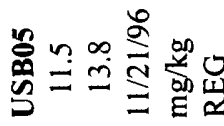

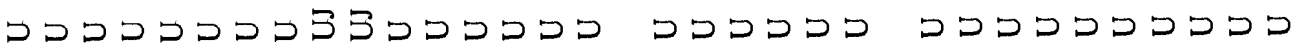

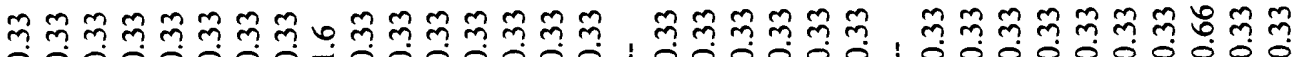

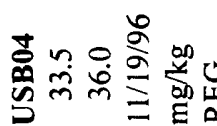

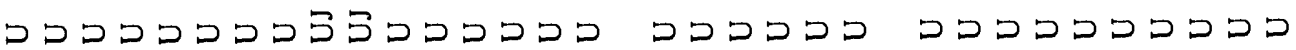

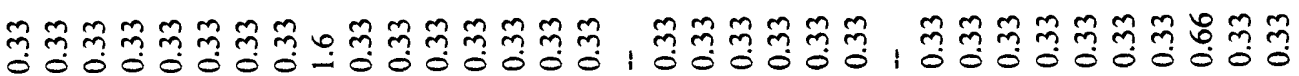

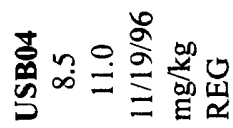

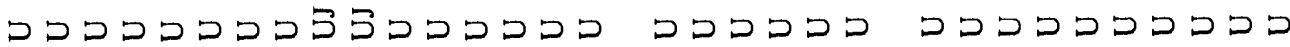

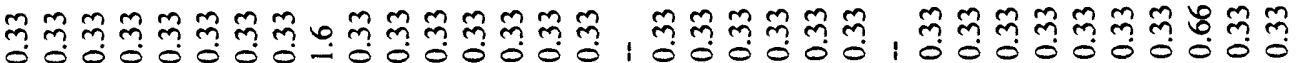

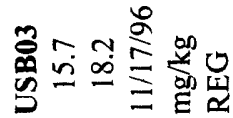

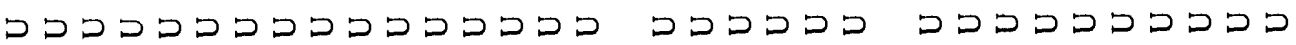

\%

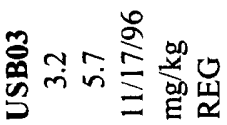

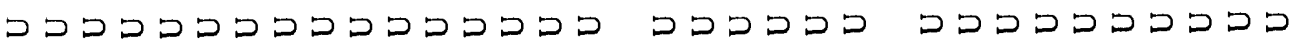

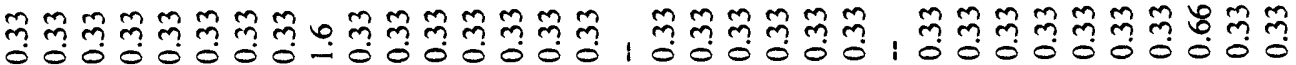

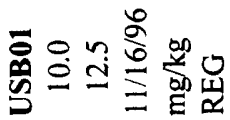

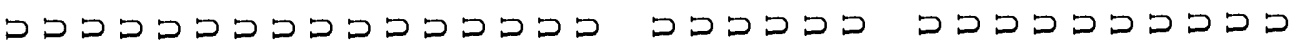

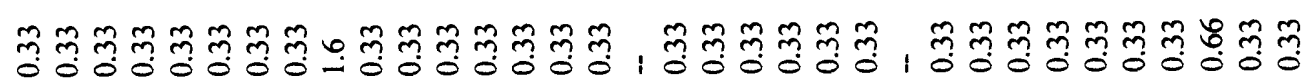

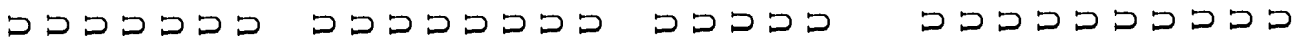

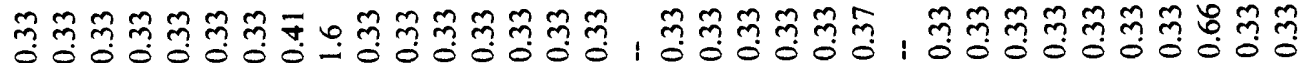
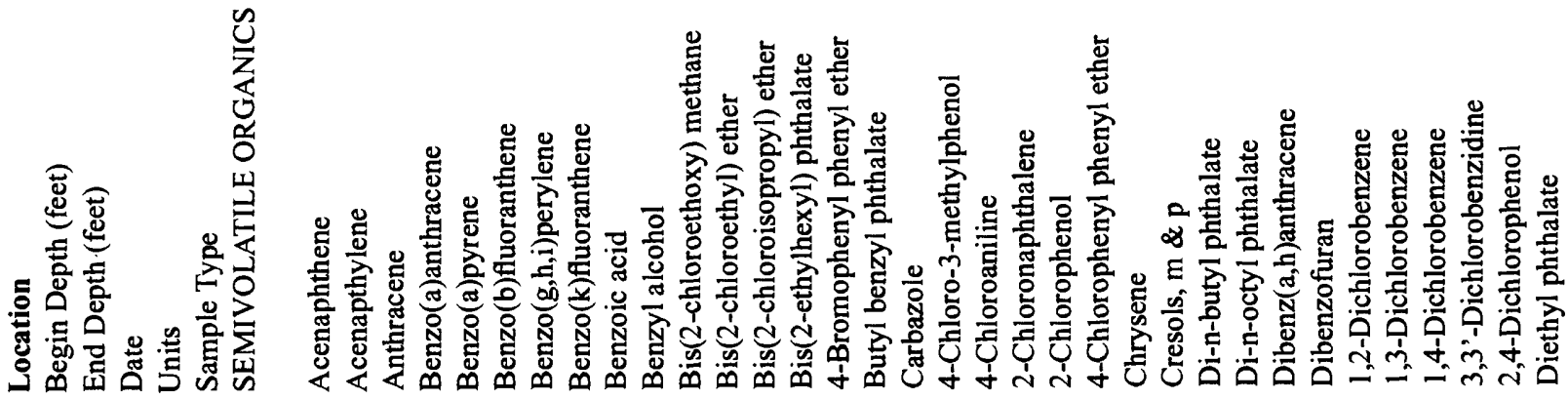


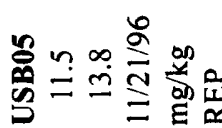

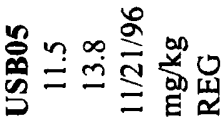

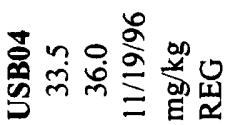

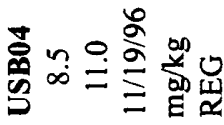

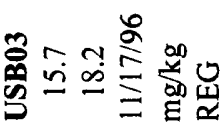

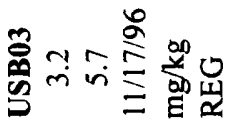

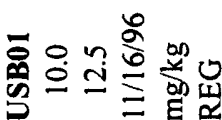

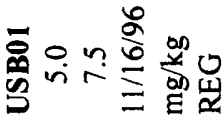

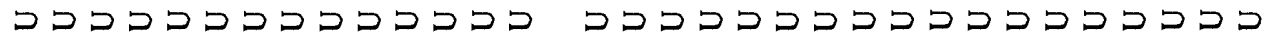

๗ิ

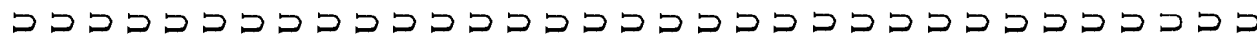

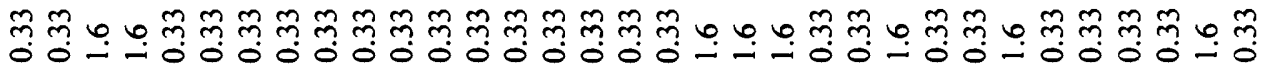

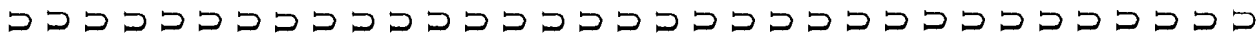

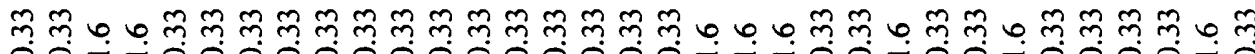

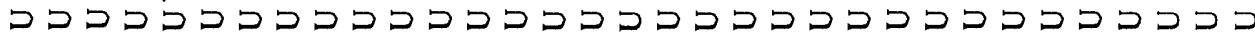

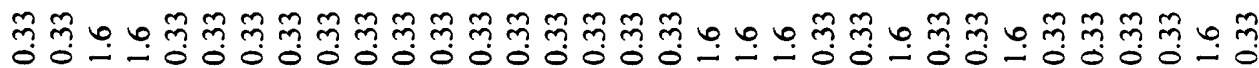

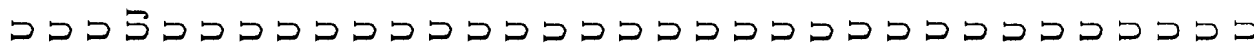

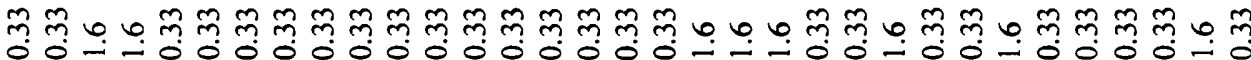

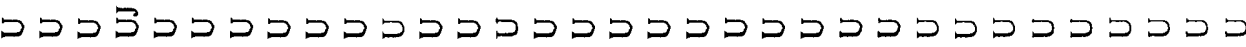

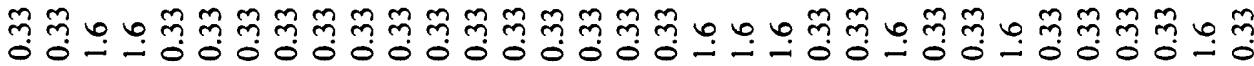

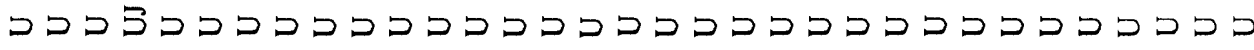

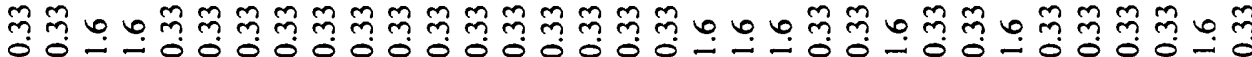

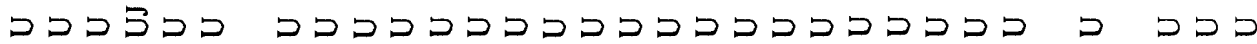

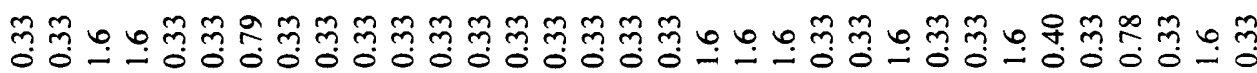
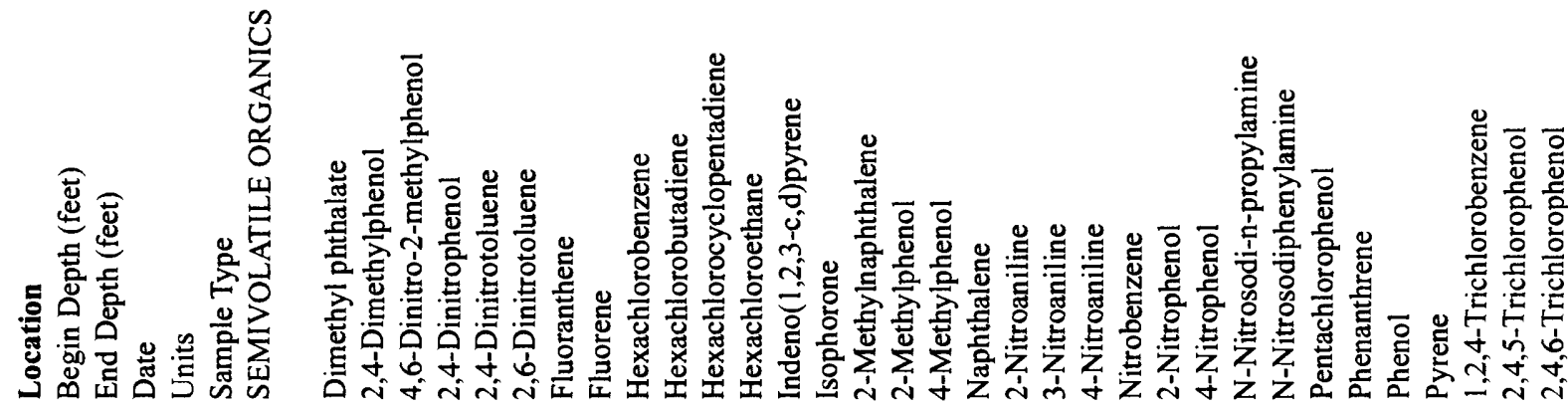


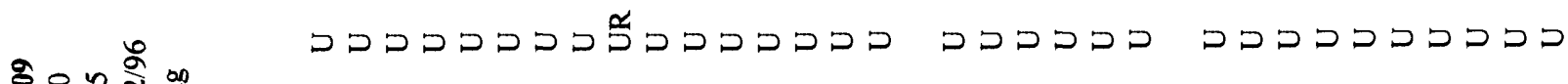

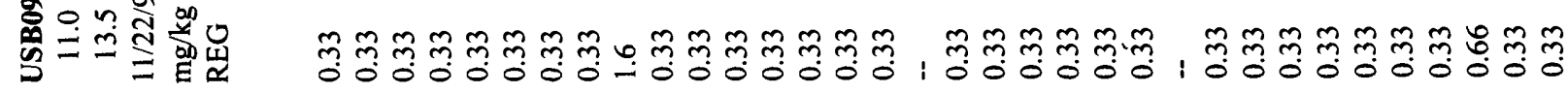

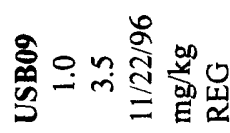

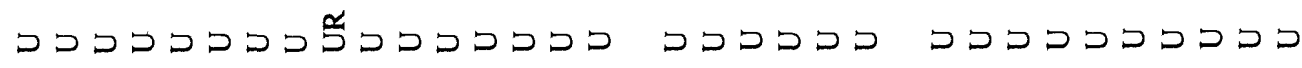

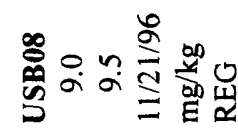

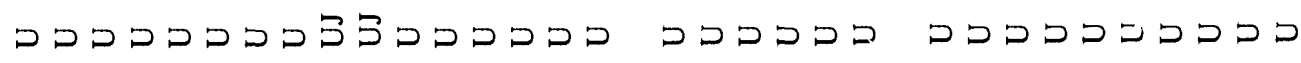

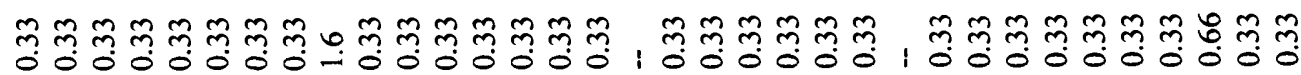

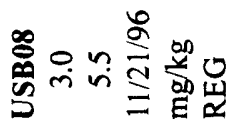

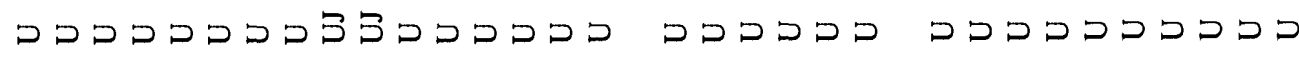

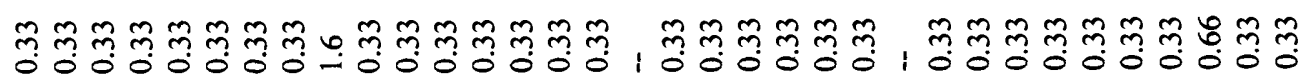

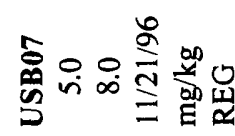

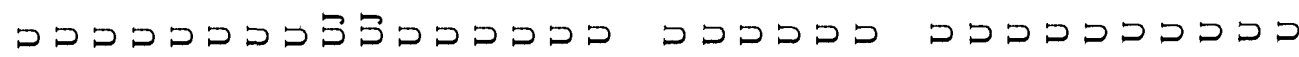

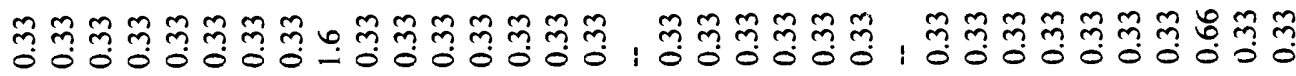

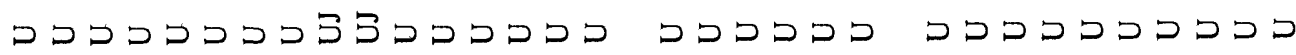

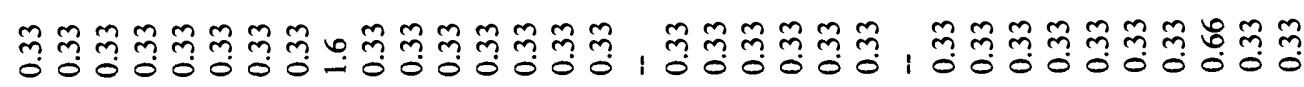

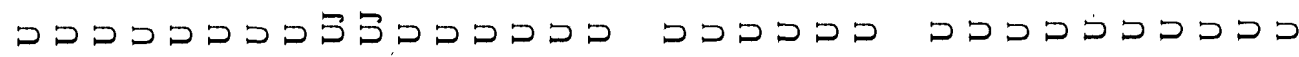

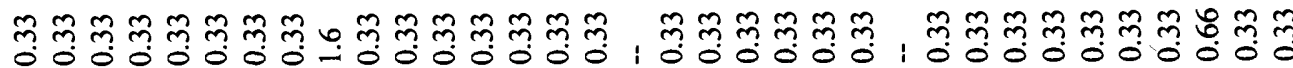

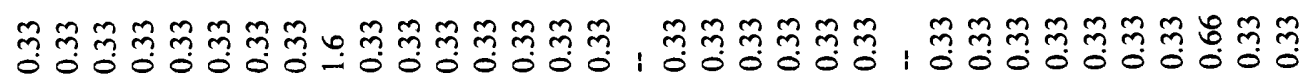
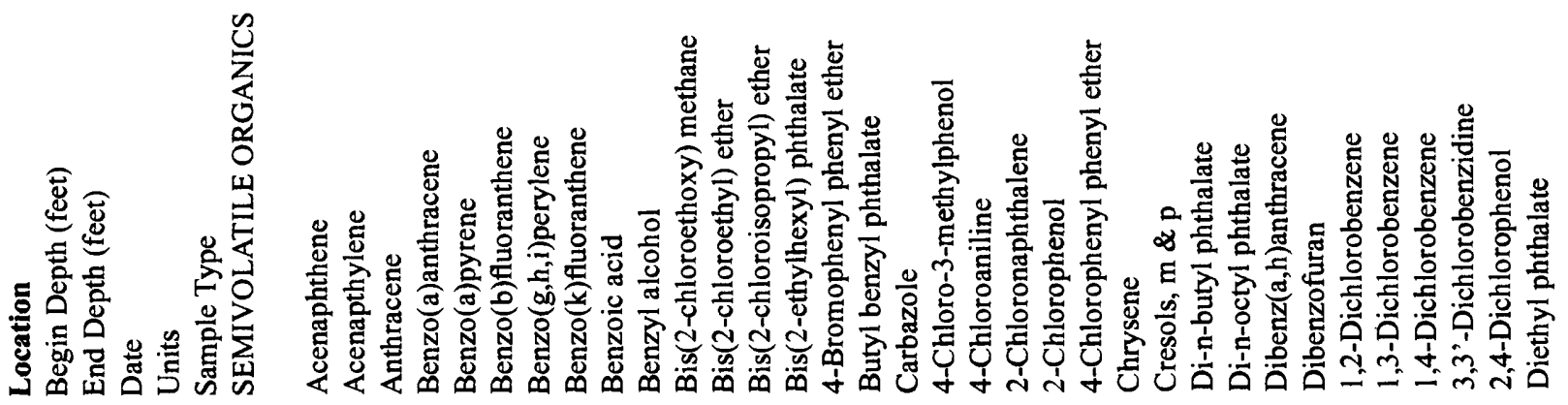


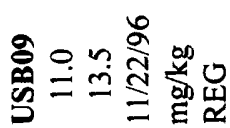

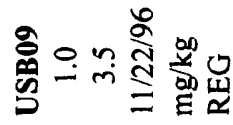

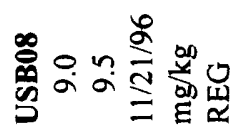

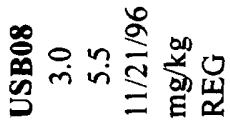

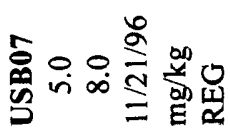

कั

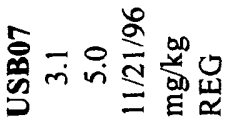

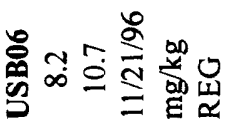

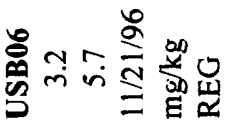

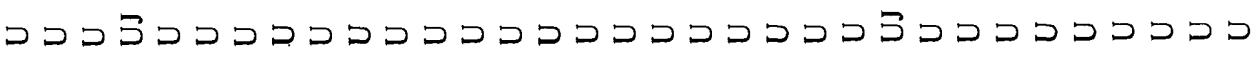

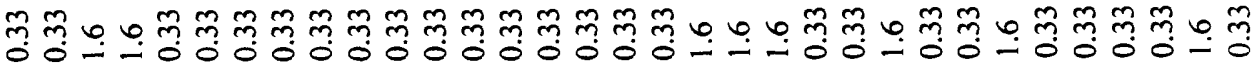

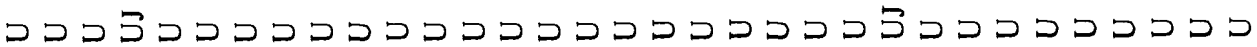

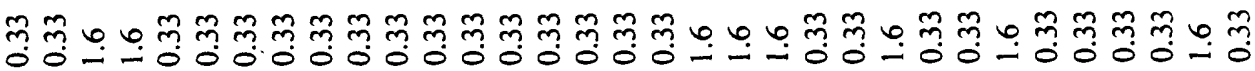

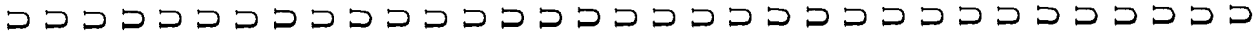

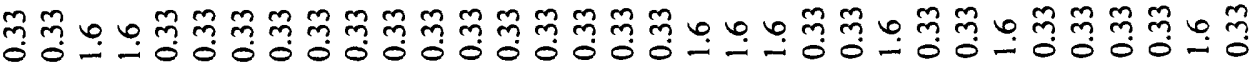

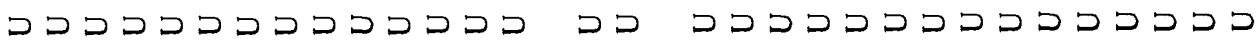
ஸ̆

১つ

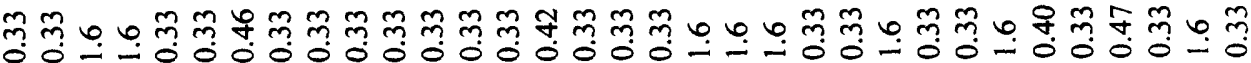

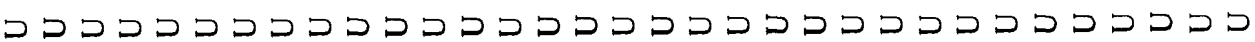

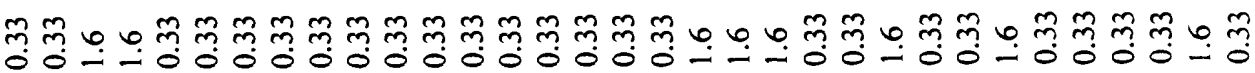

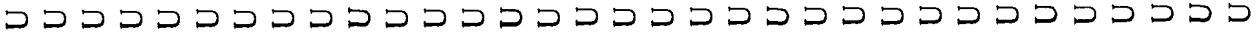

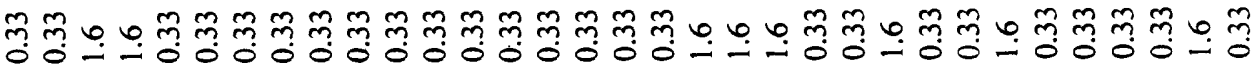

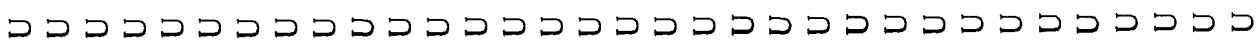

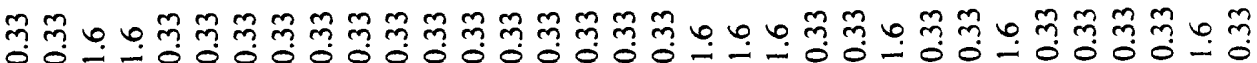

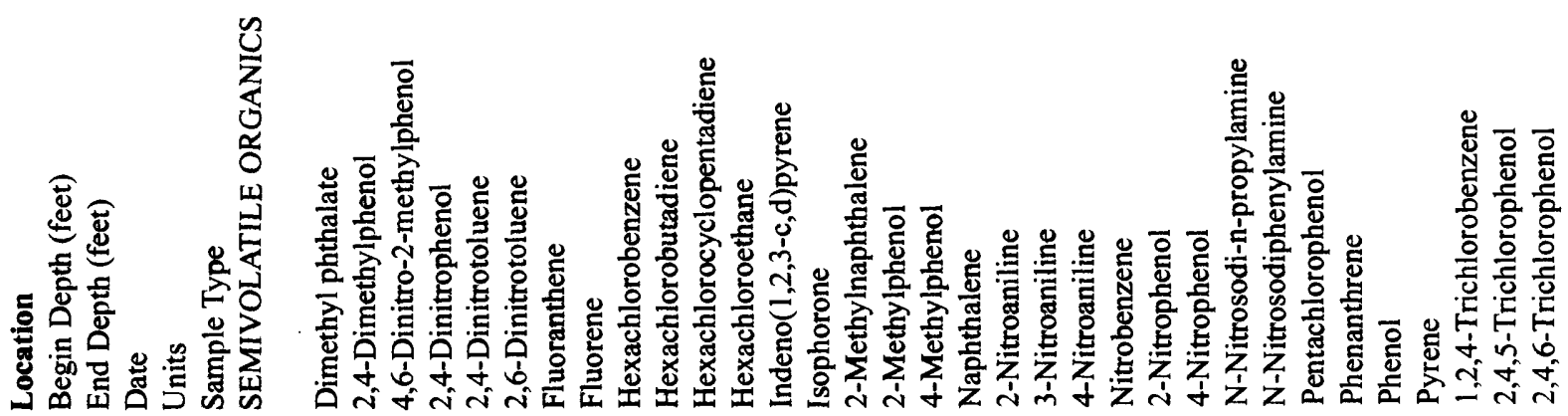




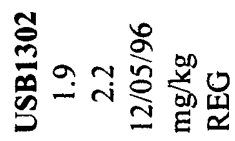

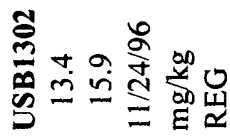

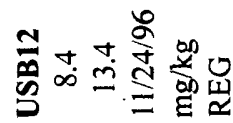

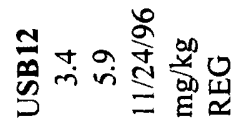

के

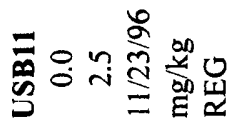

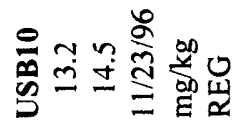

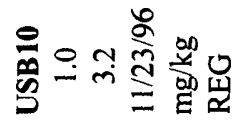

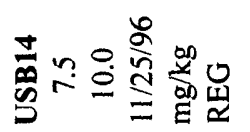

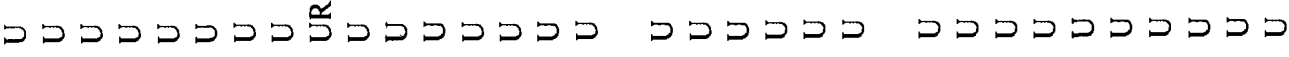

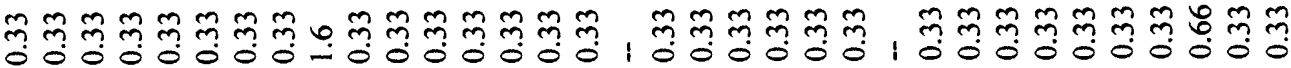

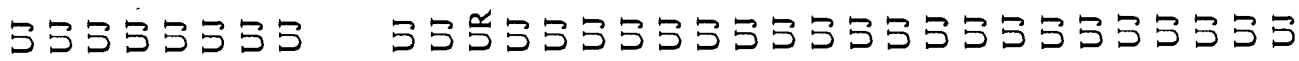

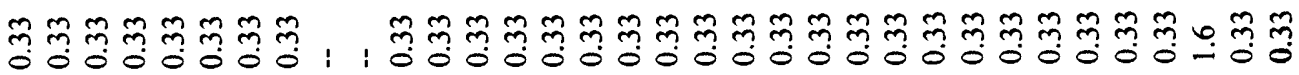

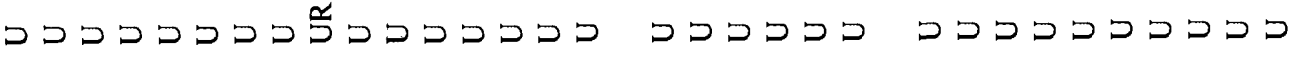

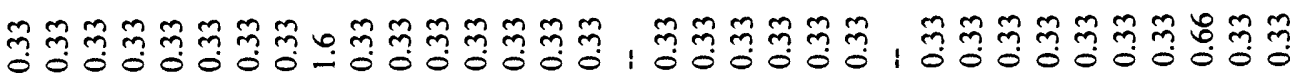

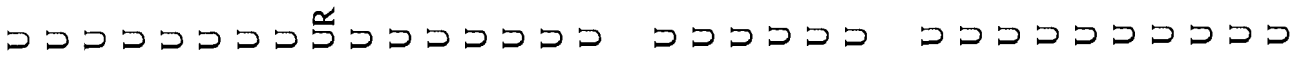

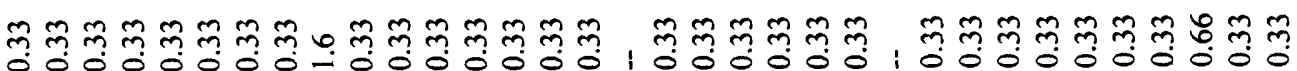

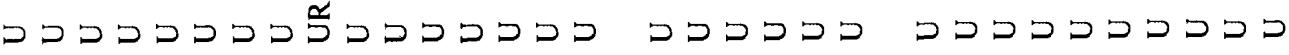

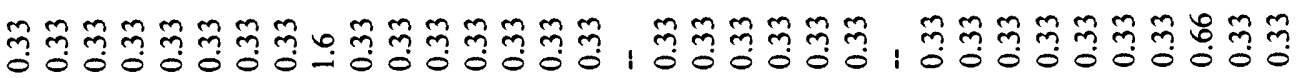

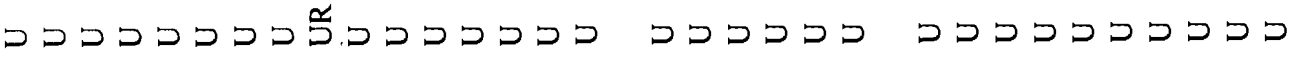

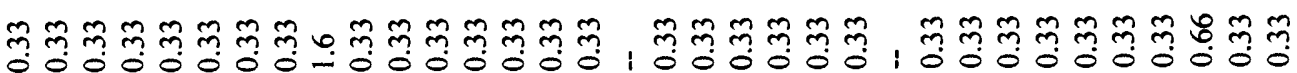

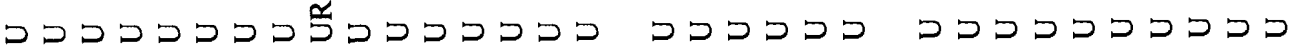

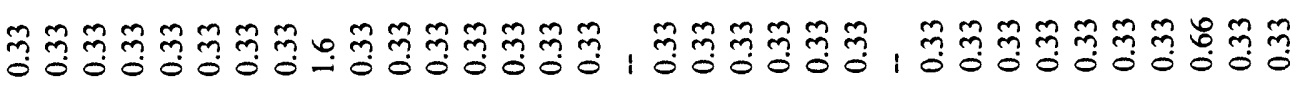
מט

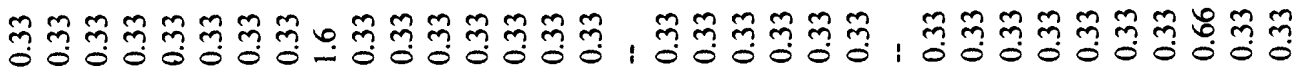

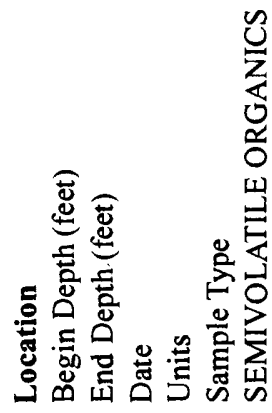

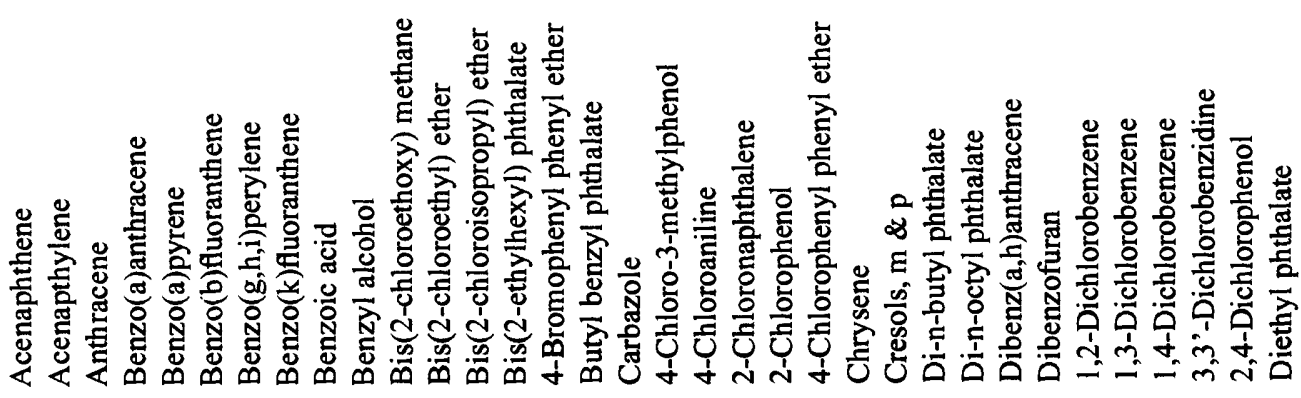

Appendix 3 


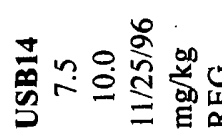

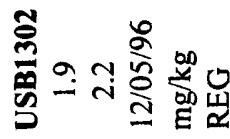

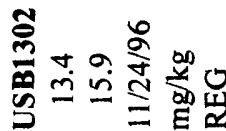

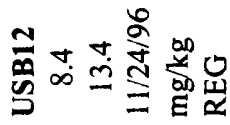

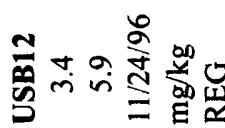

๕்

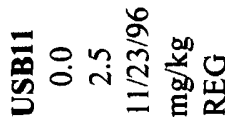

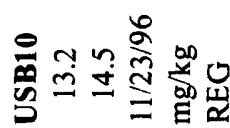

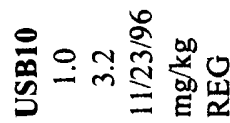

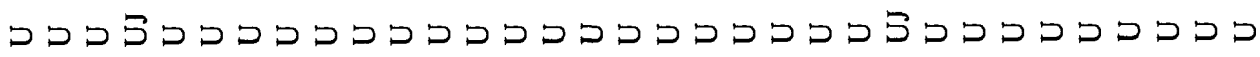

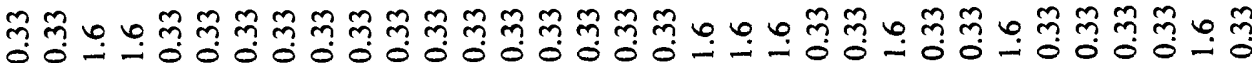

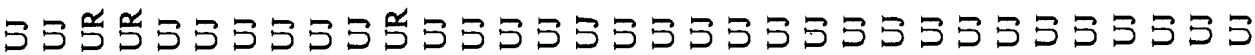

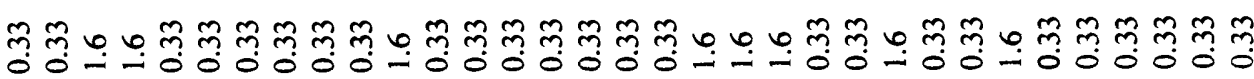

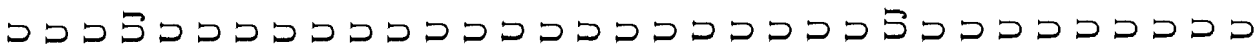

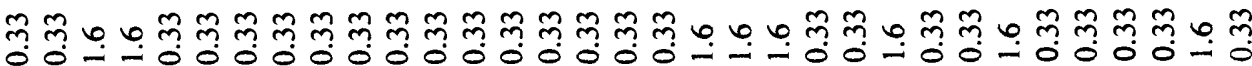

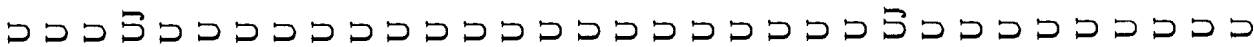

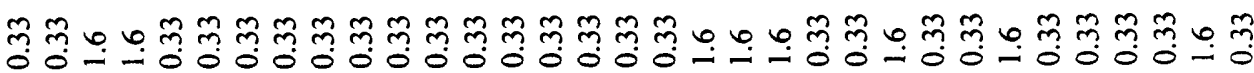

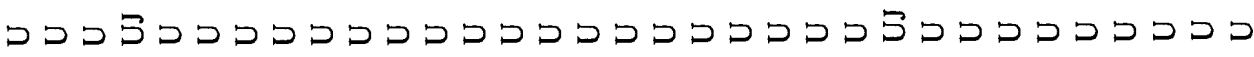

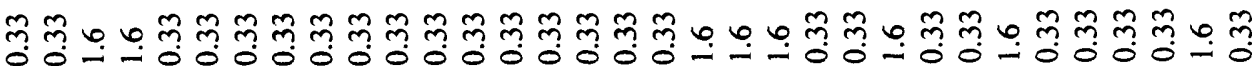

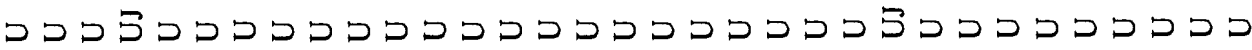

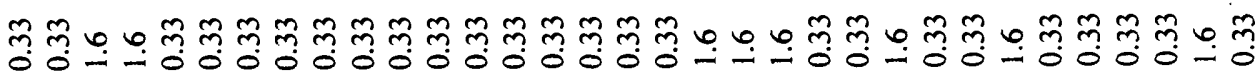

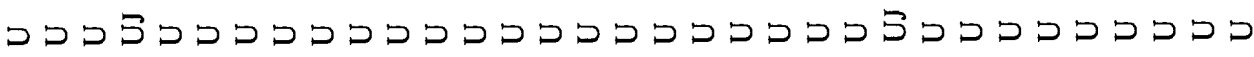

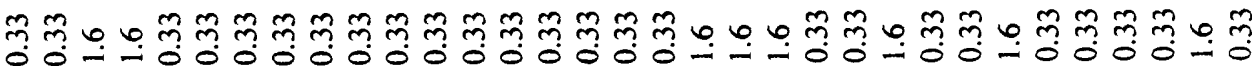

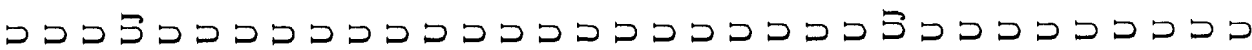

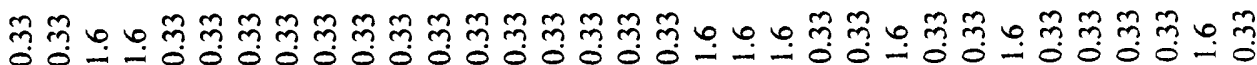
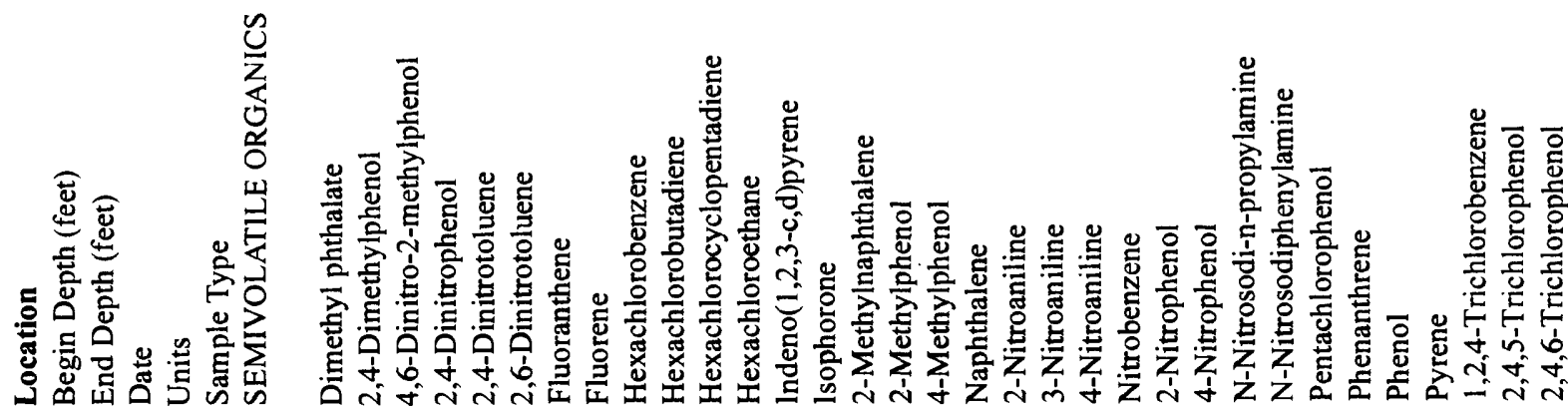


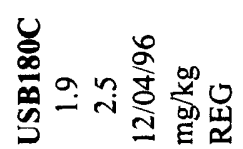

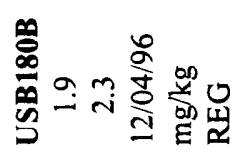

总

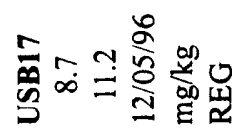

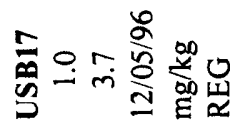

๕ั

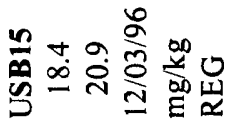

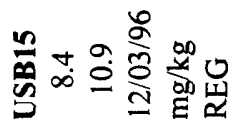

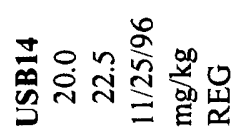

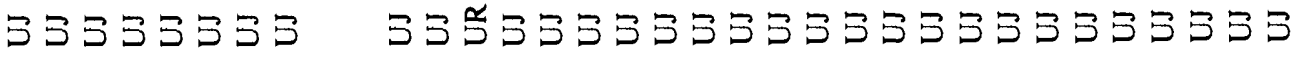

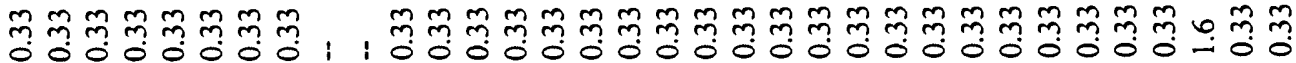

\section{รコรรรรラร}

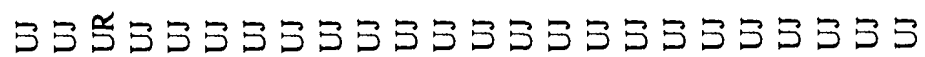

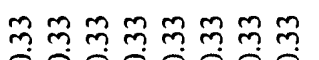
సైలె

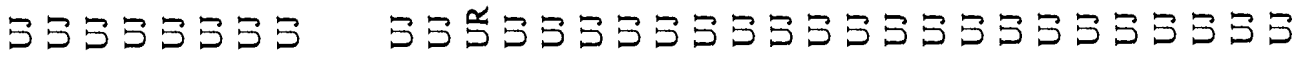

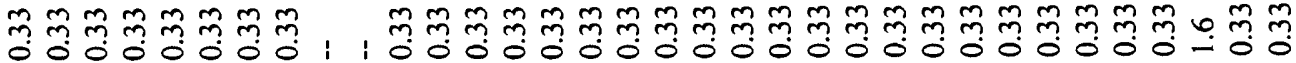

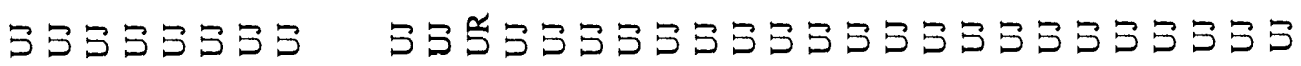

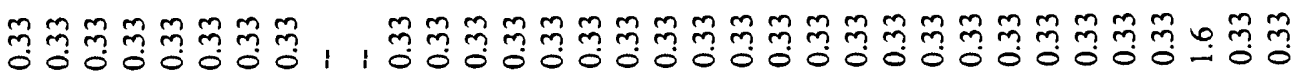

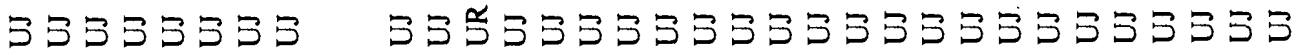

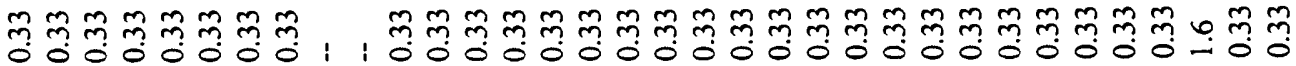

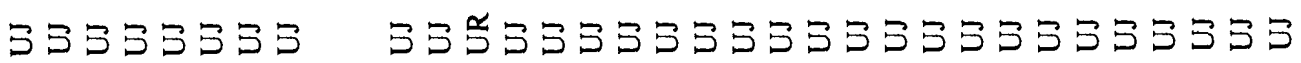

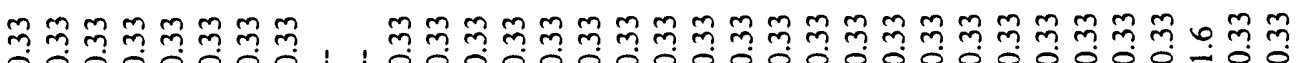

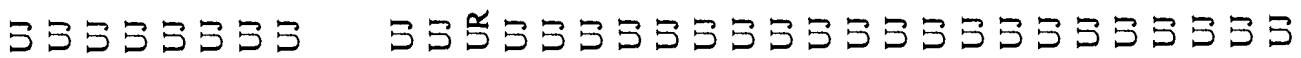

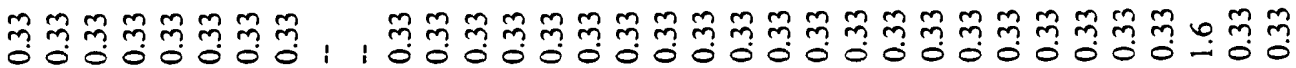

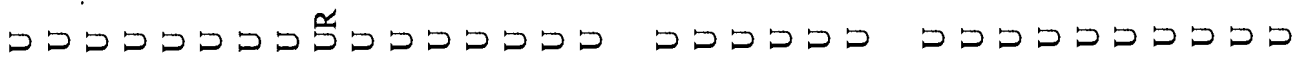

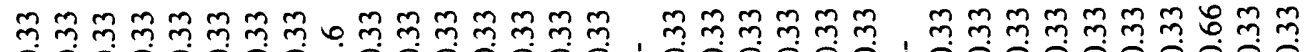

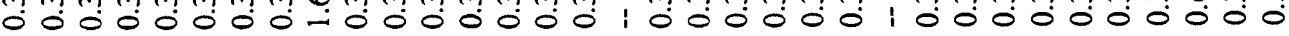

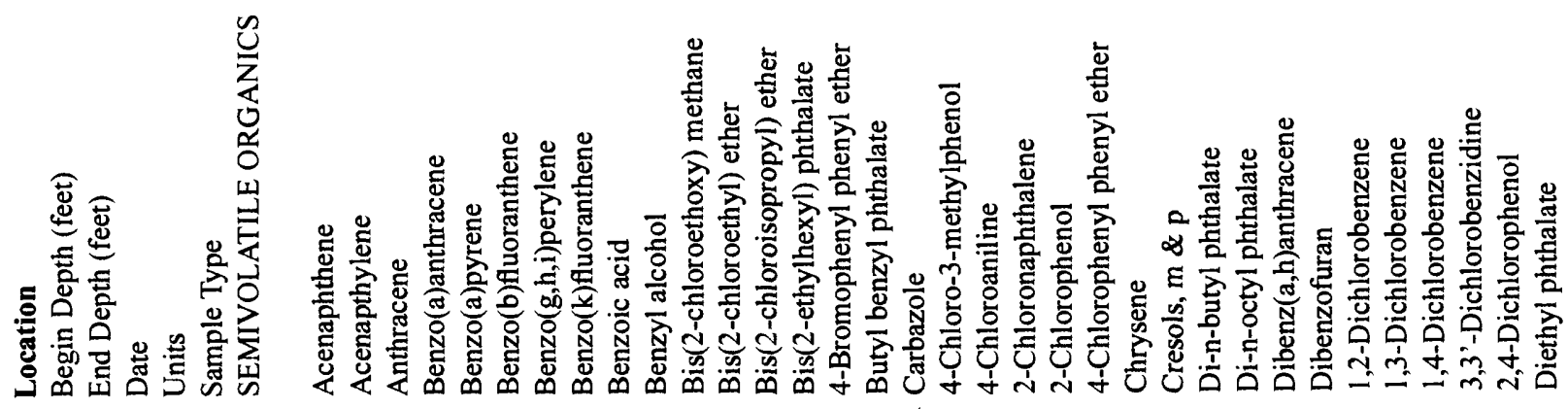




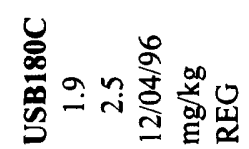

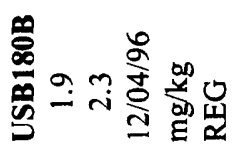

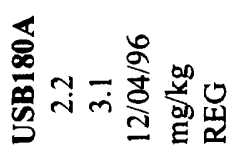

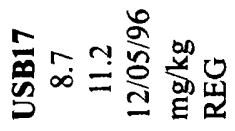

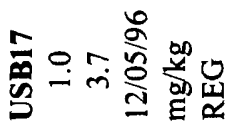

के

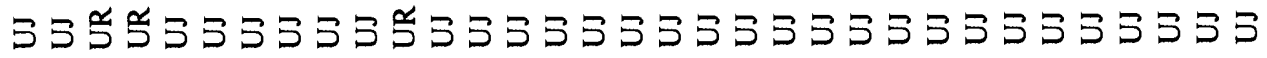

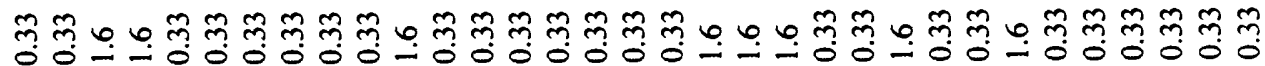

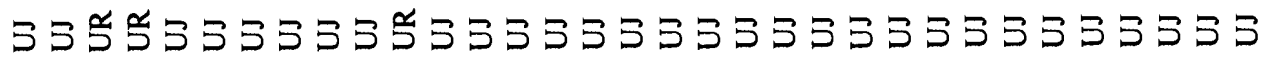

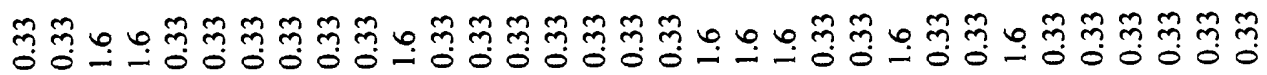

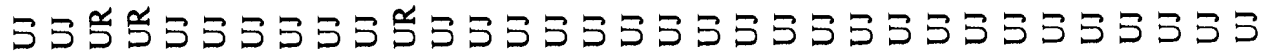

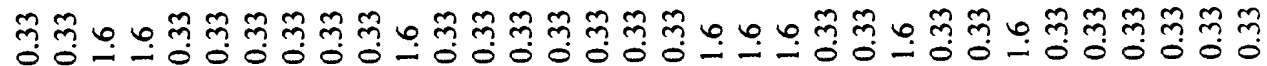

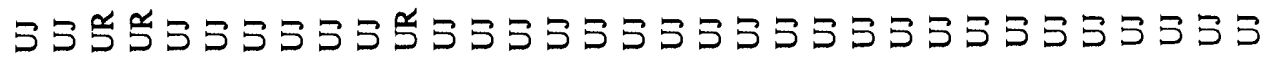

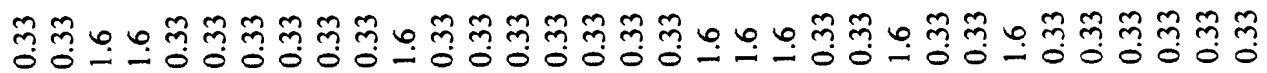

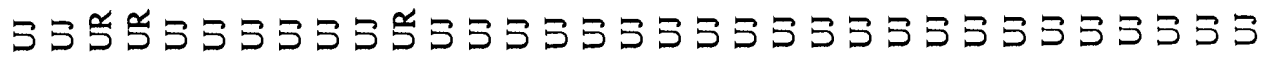

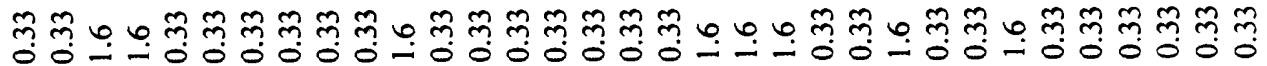

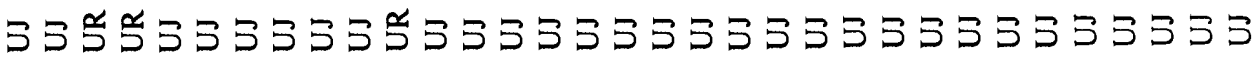

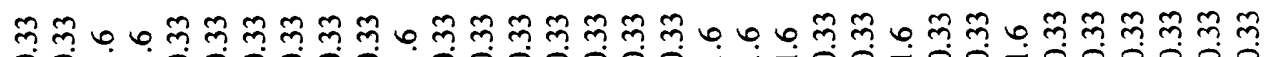

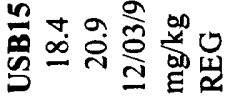

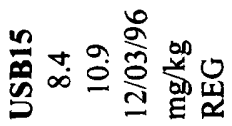

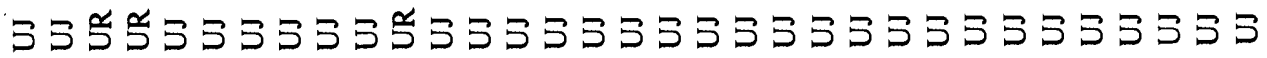

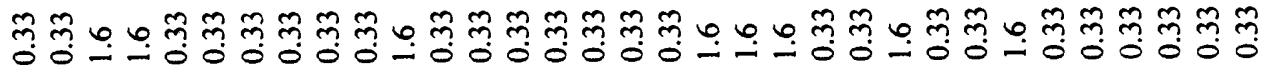

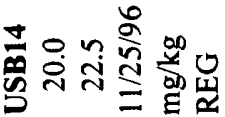
ט

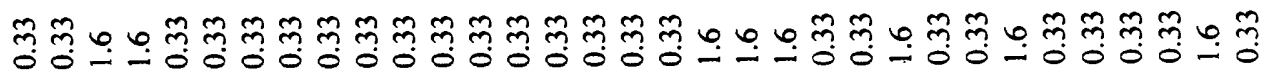
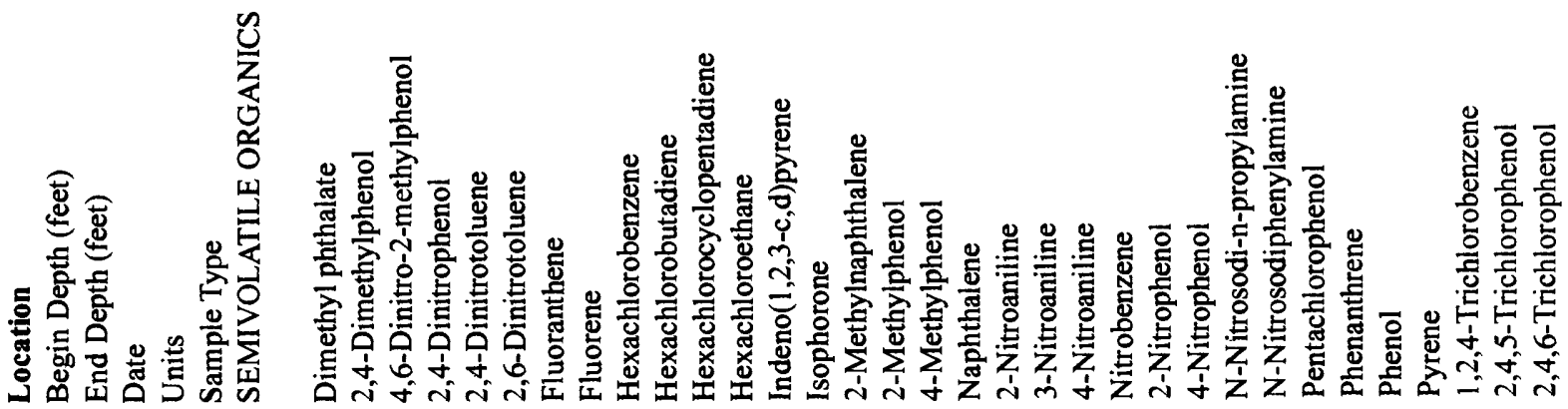


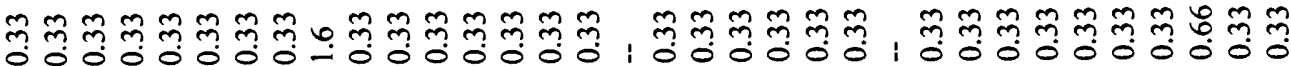

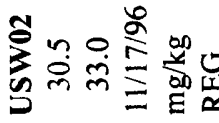

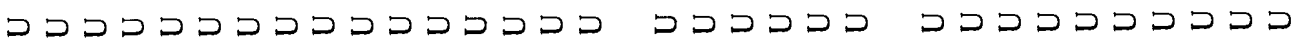

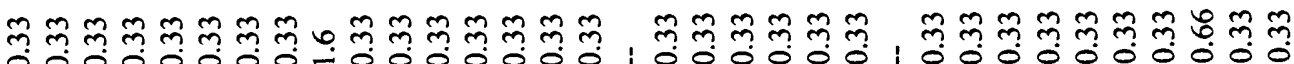

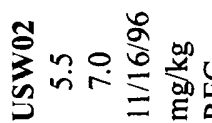

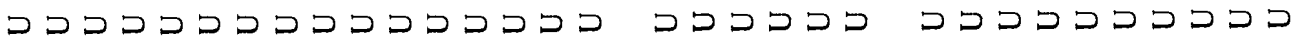

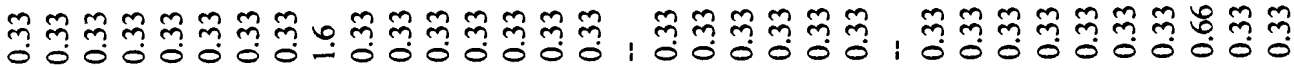

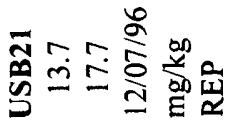

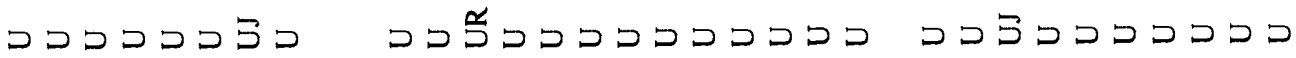

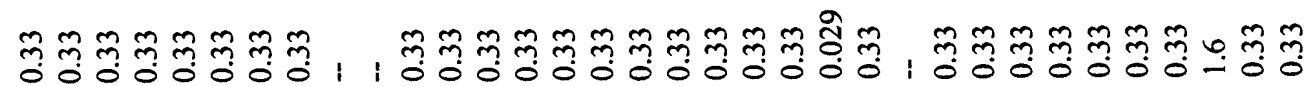

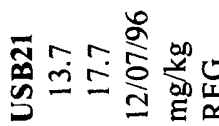

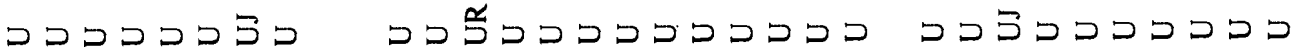

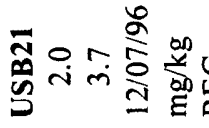

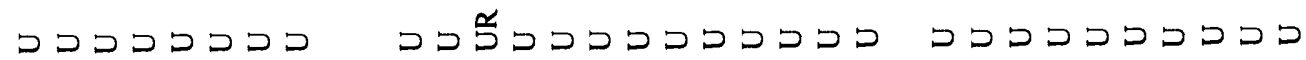

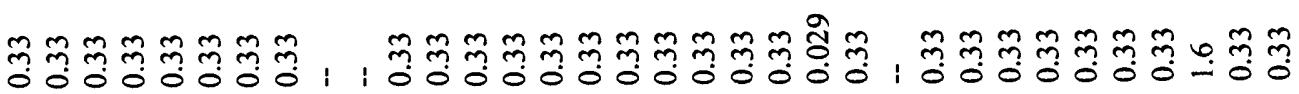

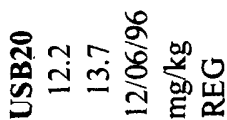

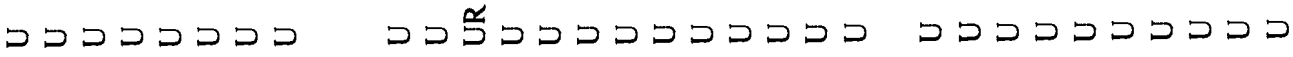

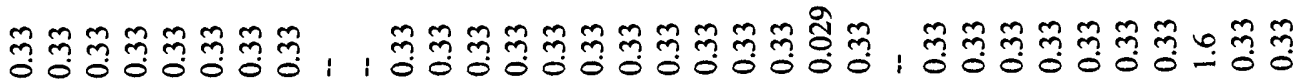

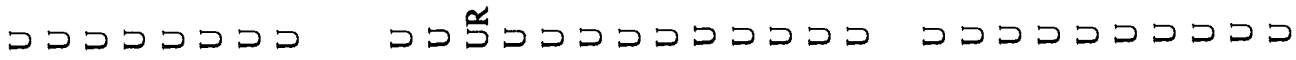

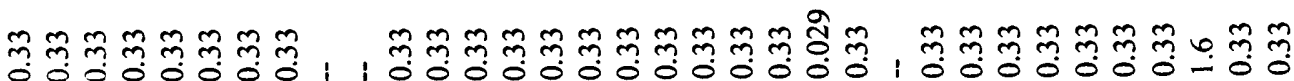
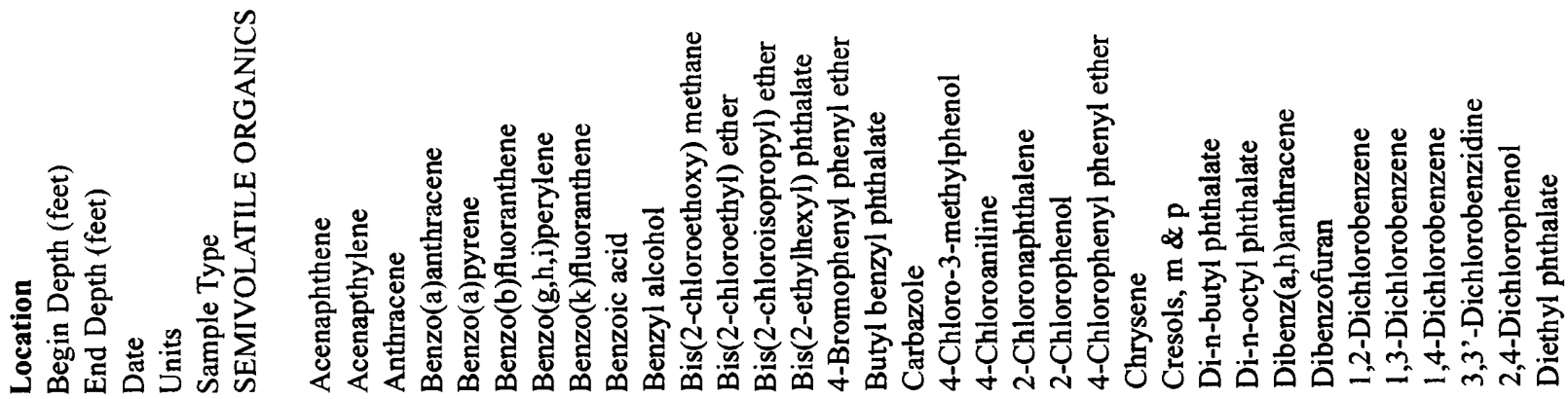


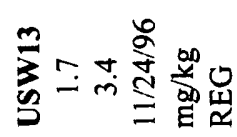

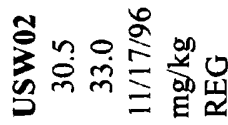

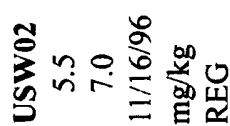

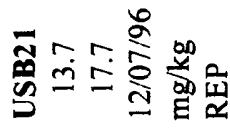

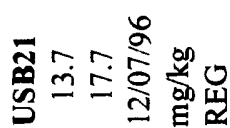

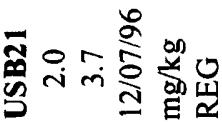

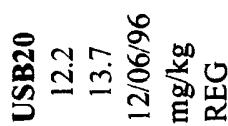

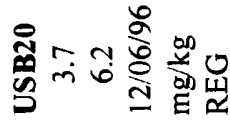

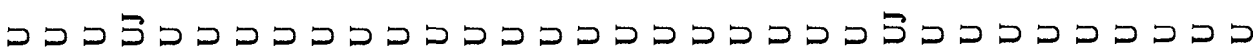

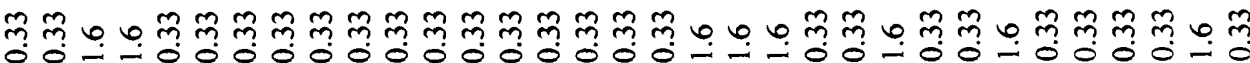

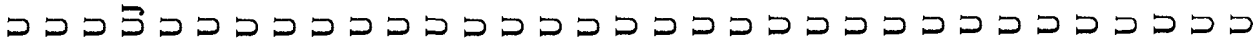

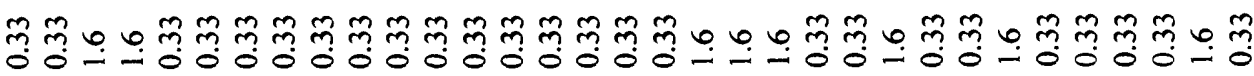

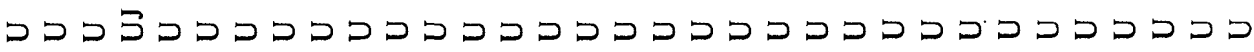

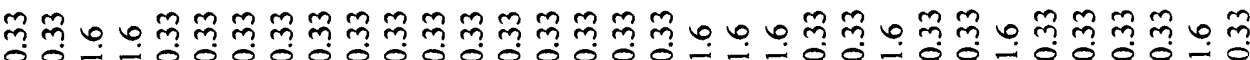
จ

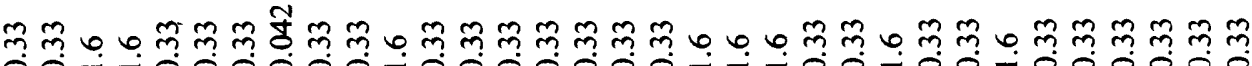

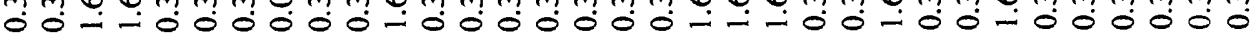

つ

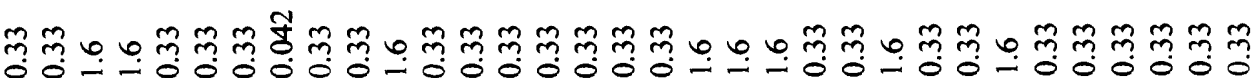

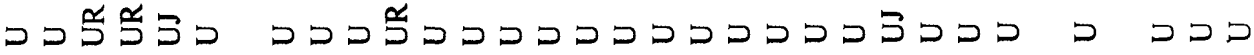

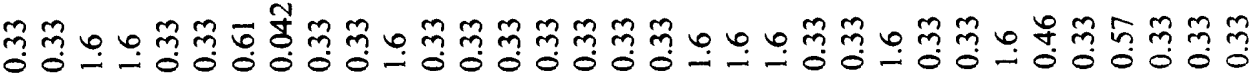

つ

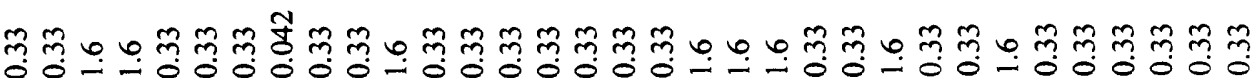
つ

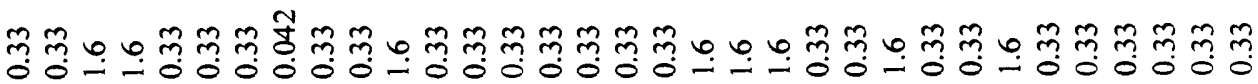
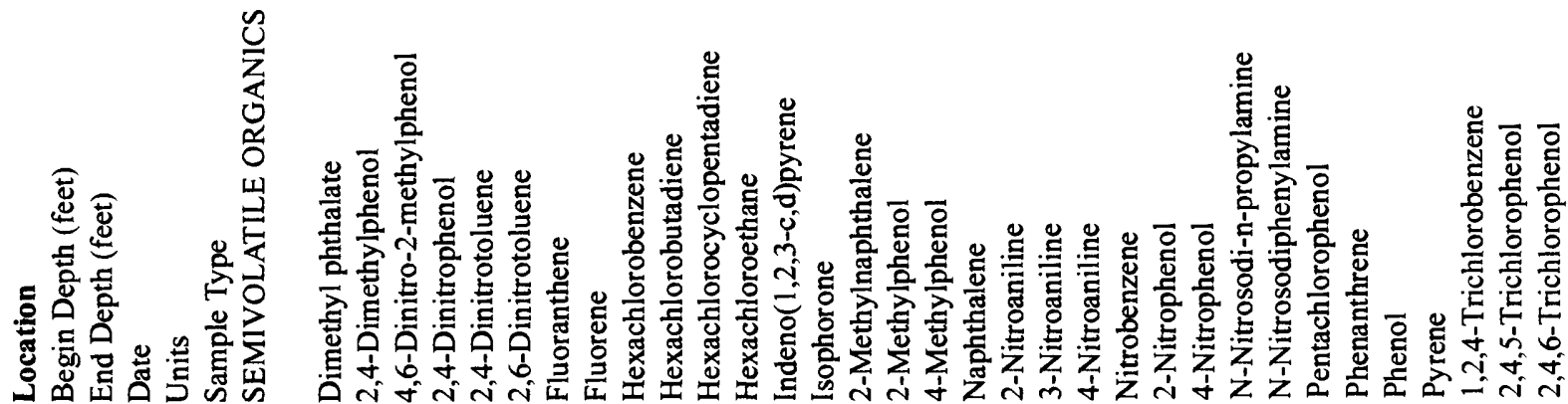
के

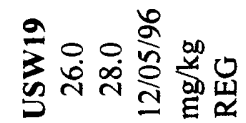

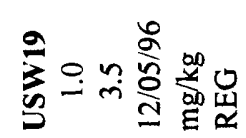

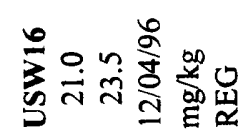

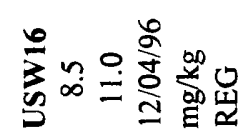

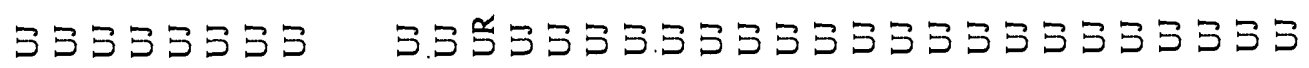

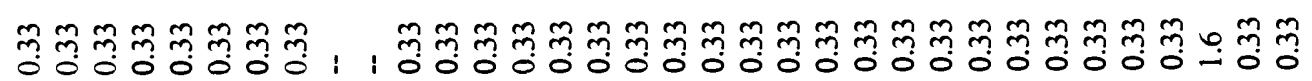

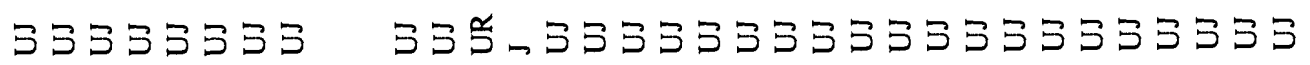

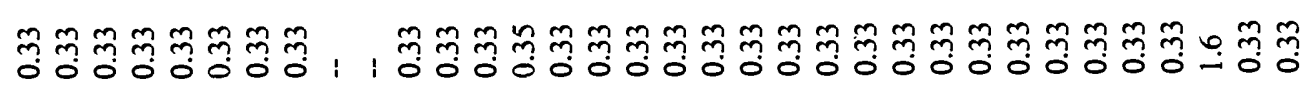

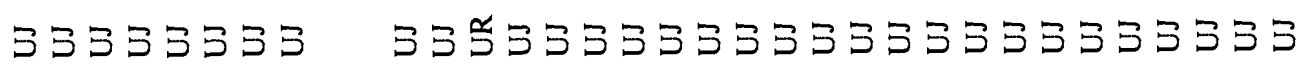

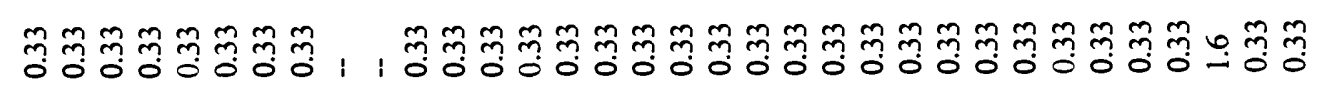

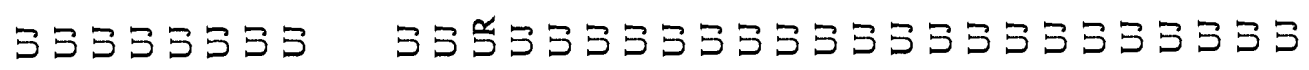

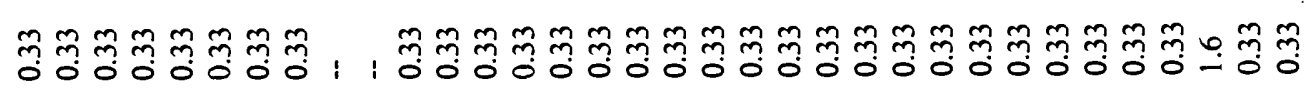

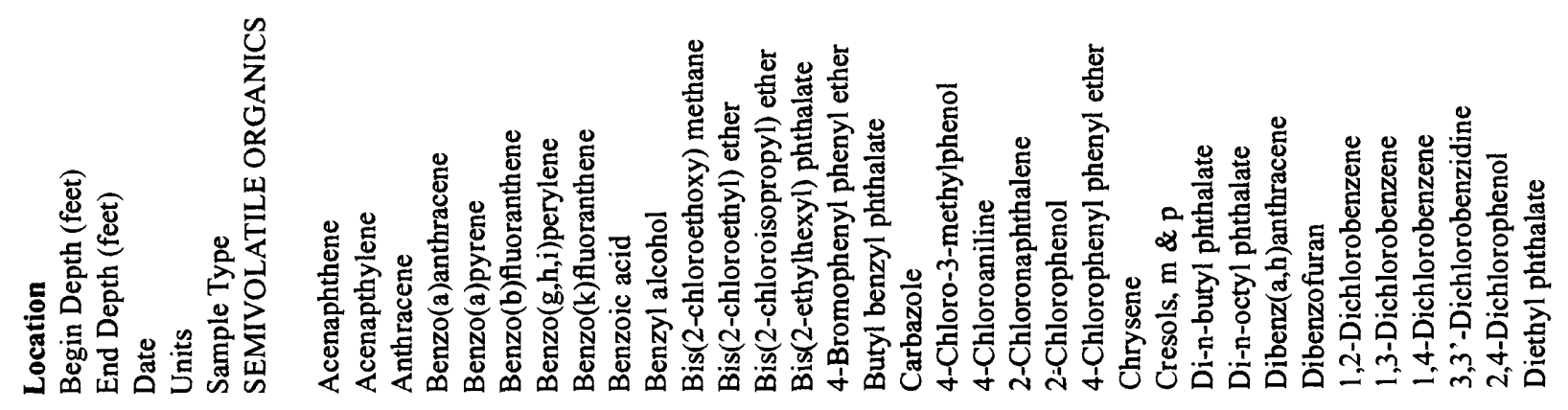




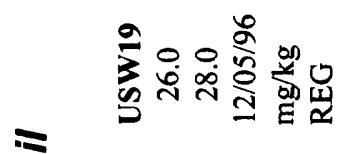

产ㅇำ

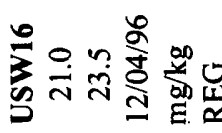

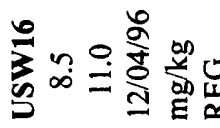

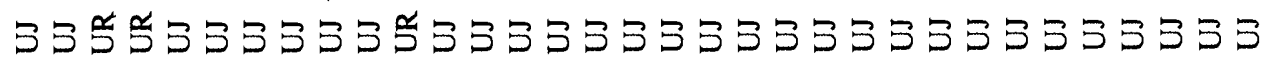

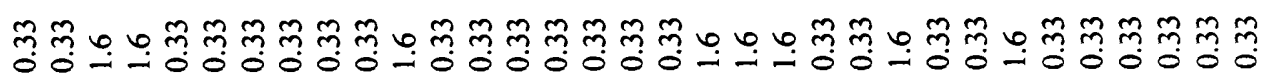
วรัง

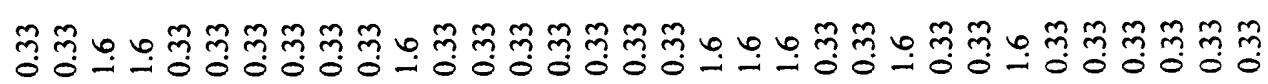

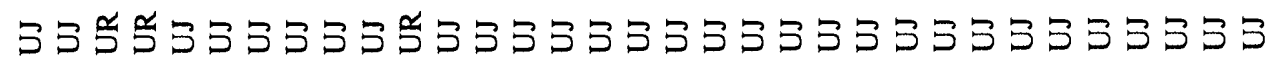

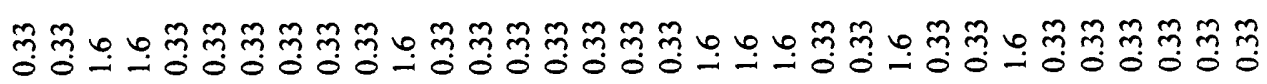

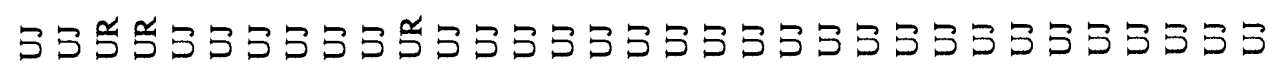

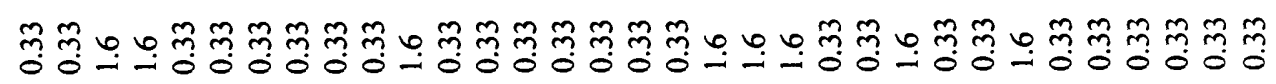

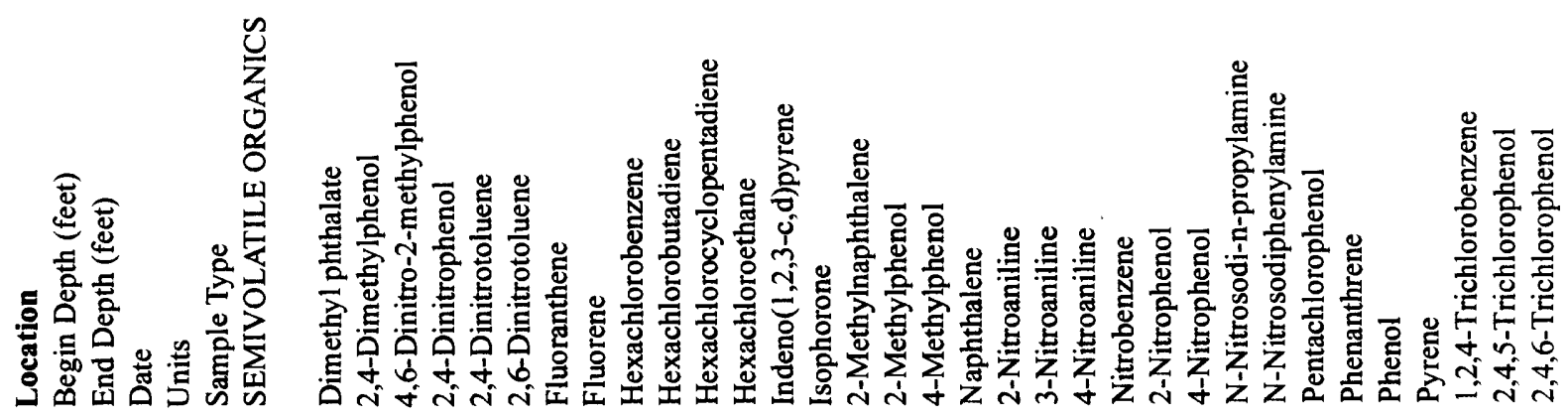




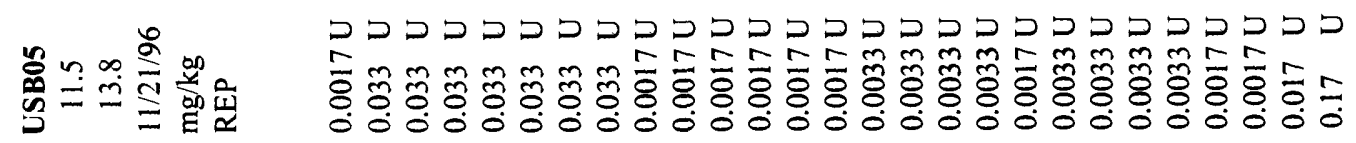

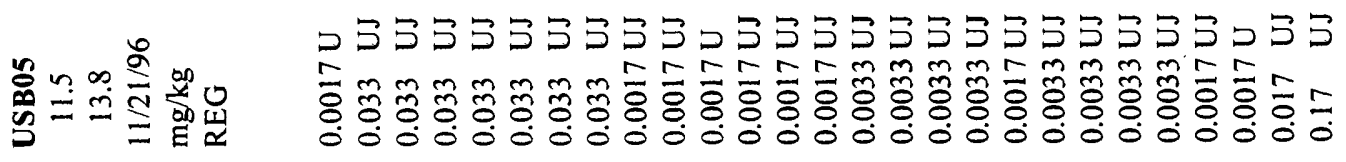

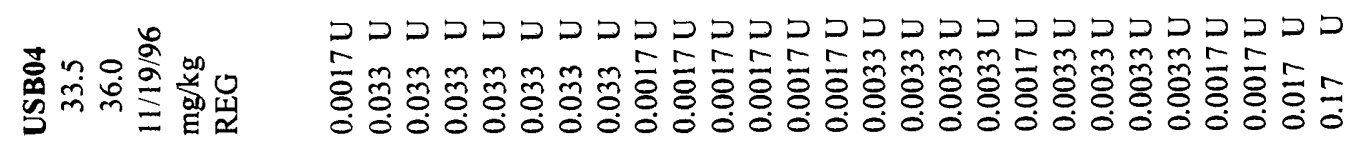

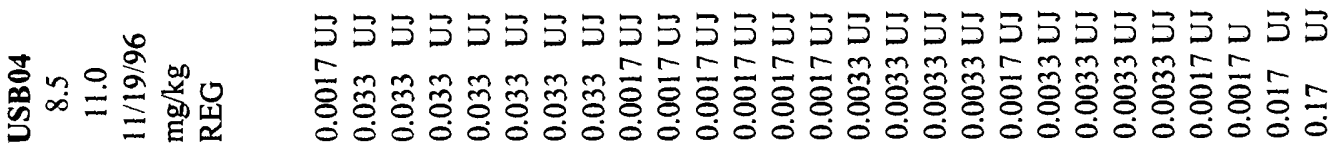

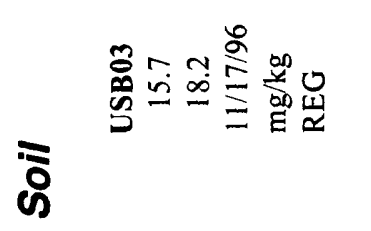
מ

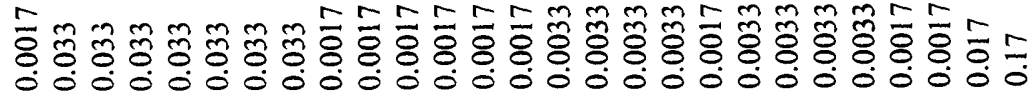

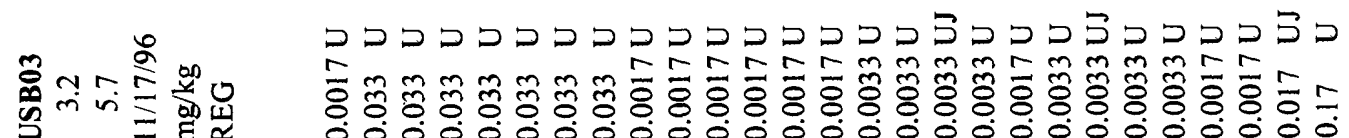

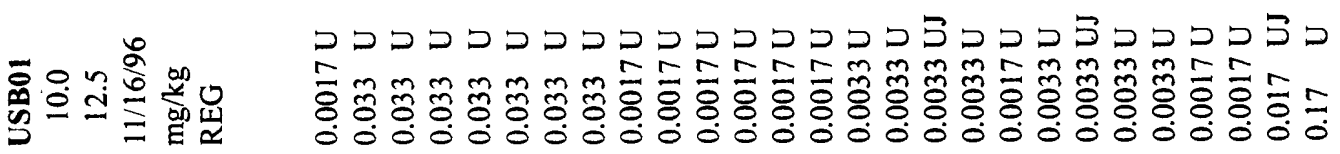

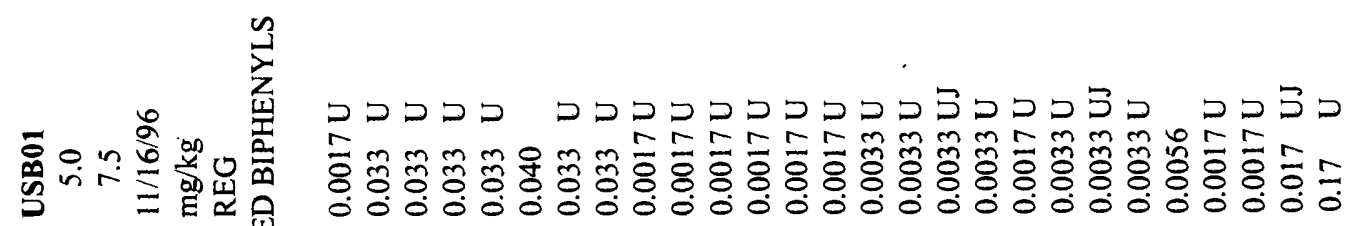
跓: 
总음

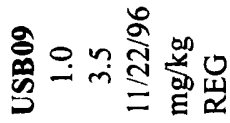

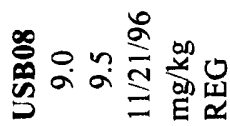

号우

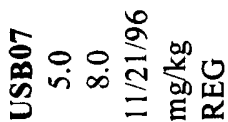

与ூ

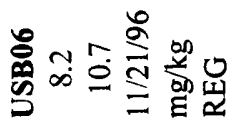

号

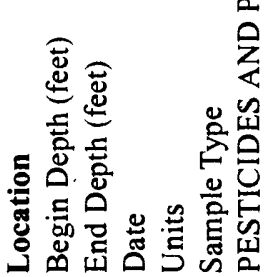

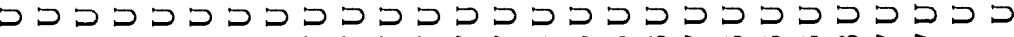
б.

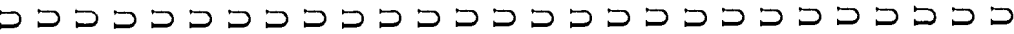

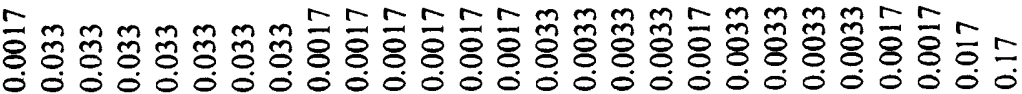

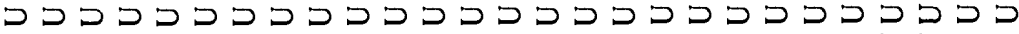
б.

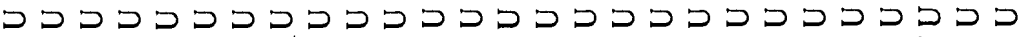

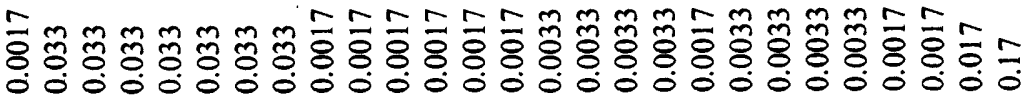

$\supset \supset \supset \supset \supset \supset \supset \supset \supset \supset \supset \supset \supset \supset \supset \supset \supset \supset \supset \supset \supset \supset \supset \supset \supset \supset \supset$

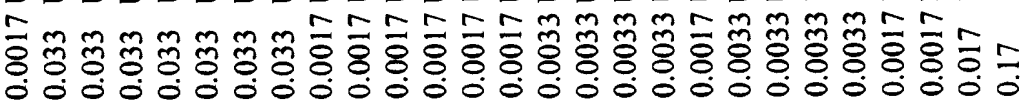

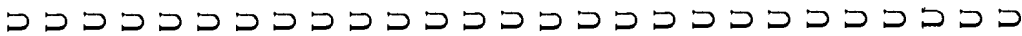

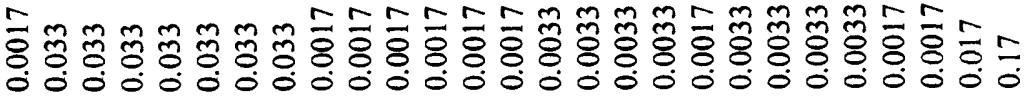

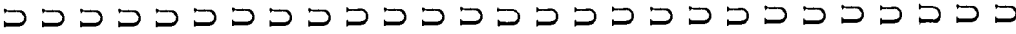

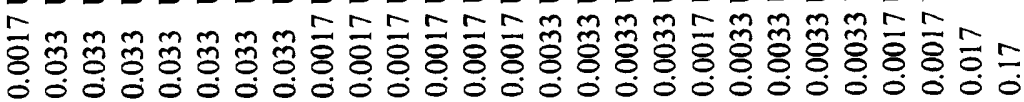

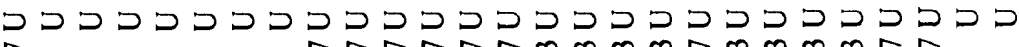

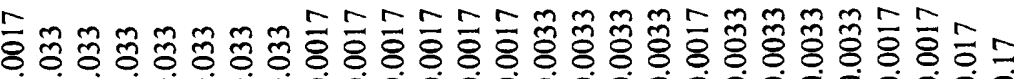

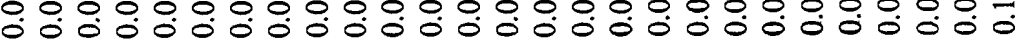

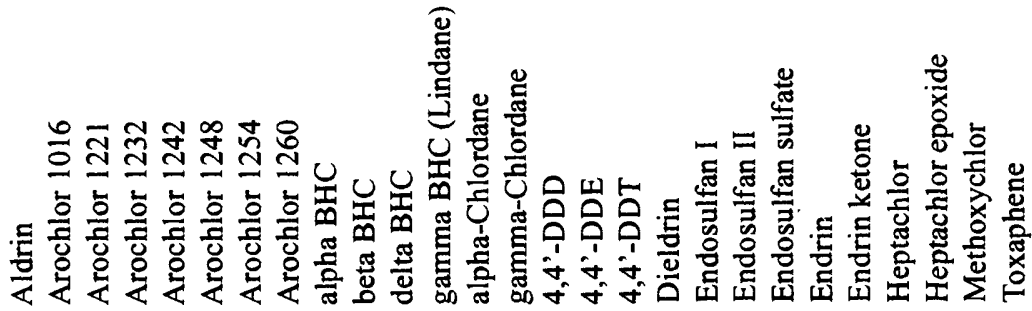




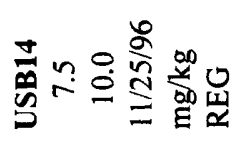

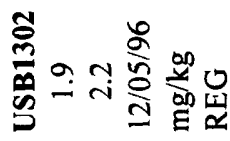

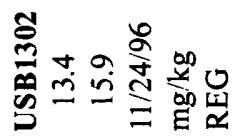

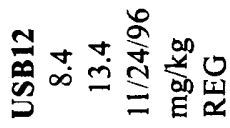

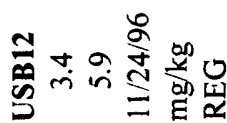

के

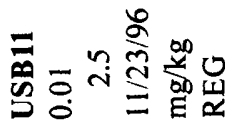

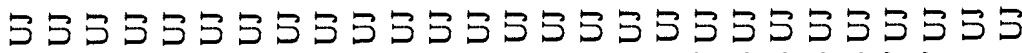

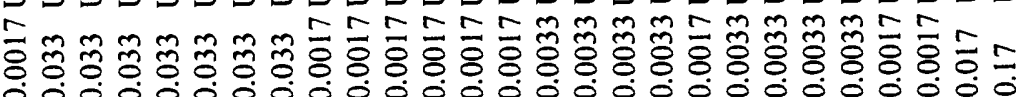

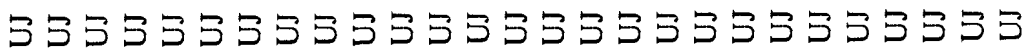

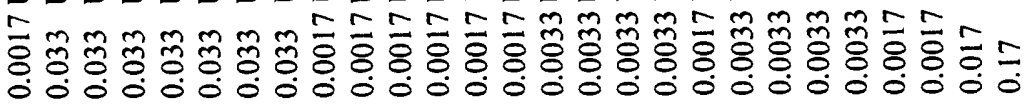

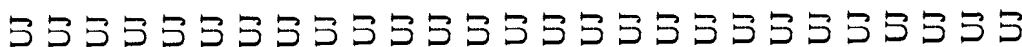

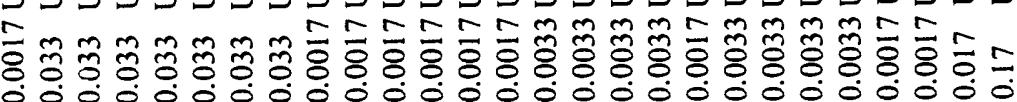

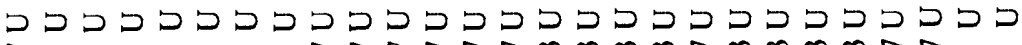

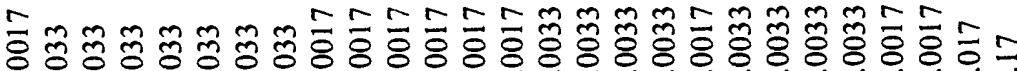
5.

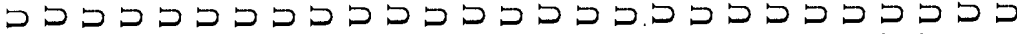

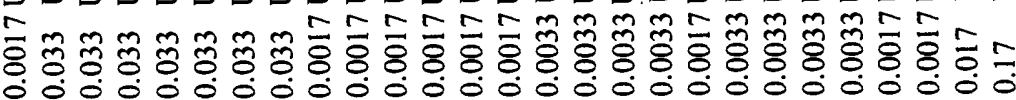

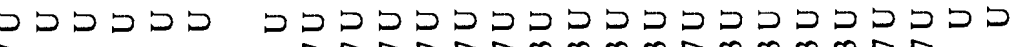

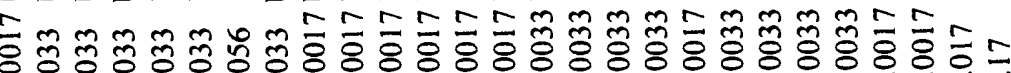

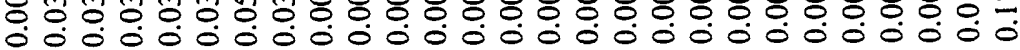

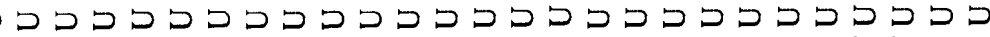

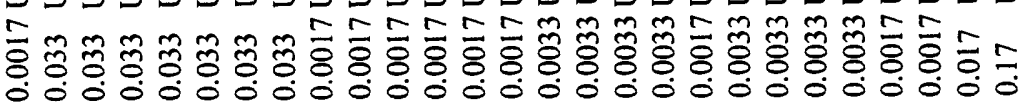

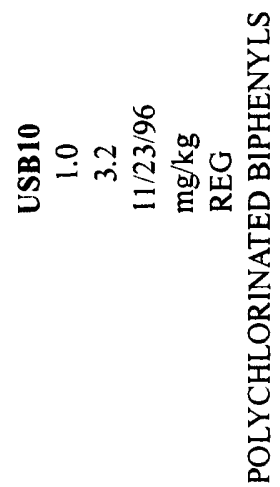

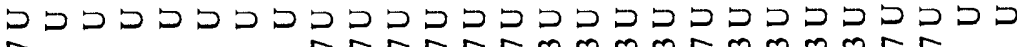

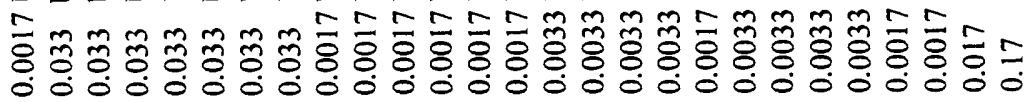
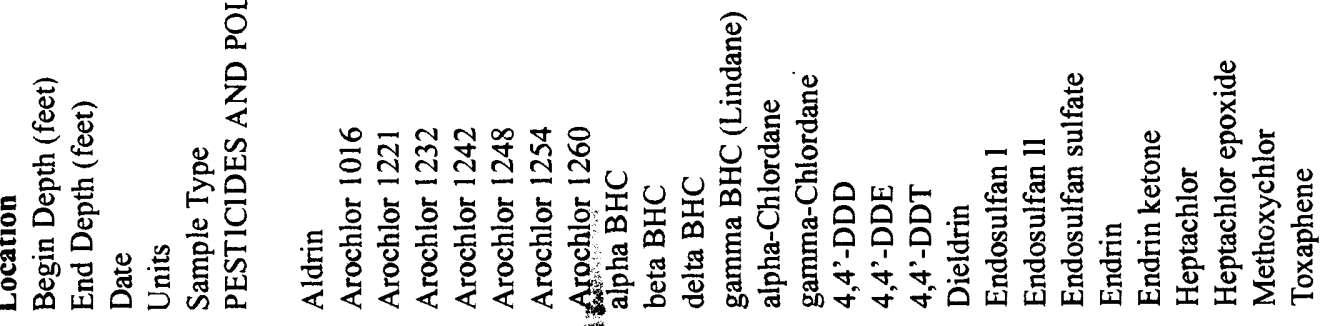


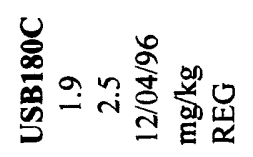

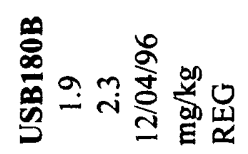

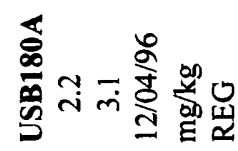

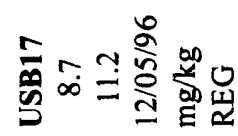

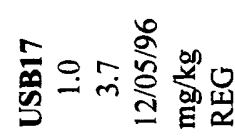

के

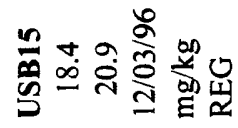

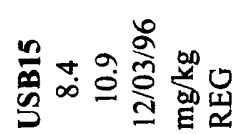

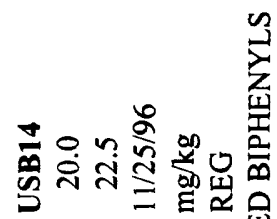

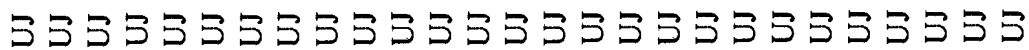

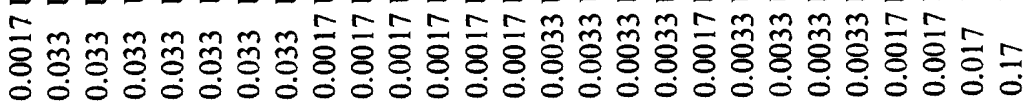

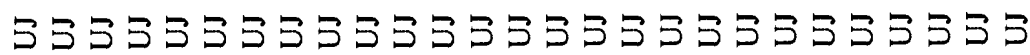
б

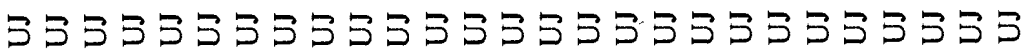

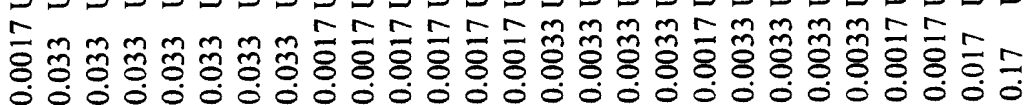

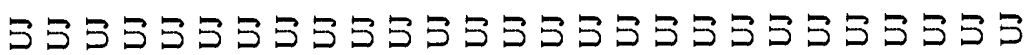

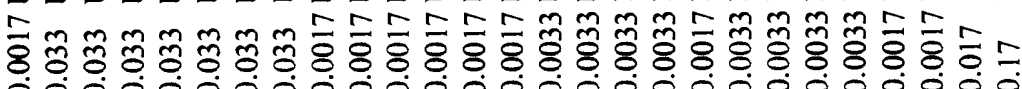

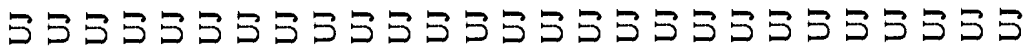

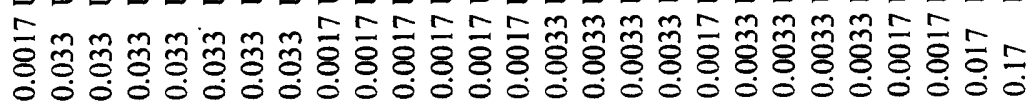

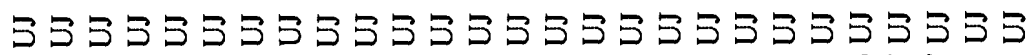

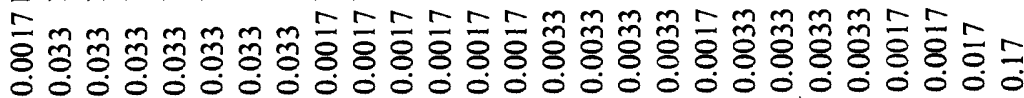

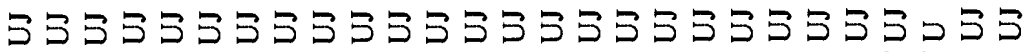

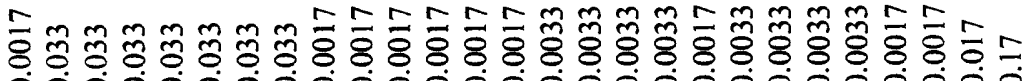

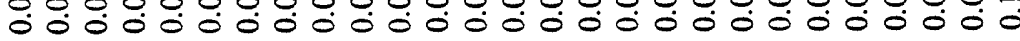

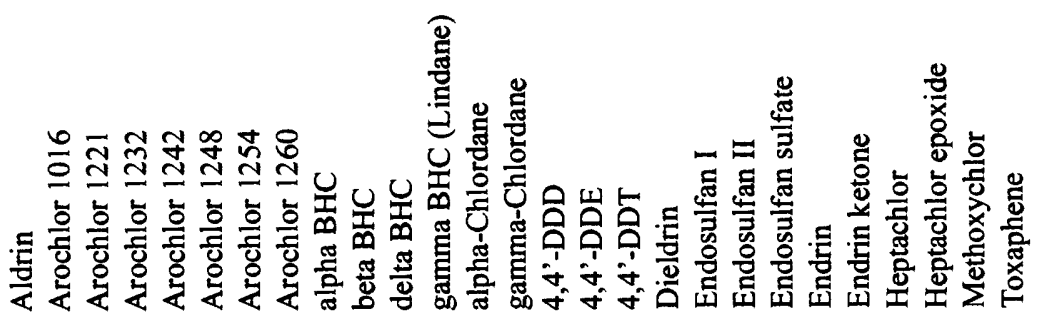




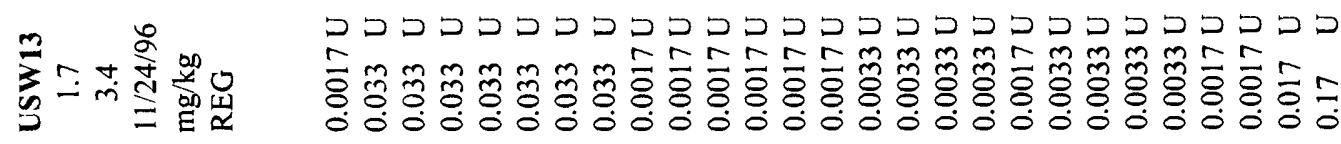

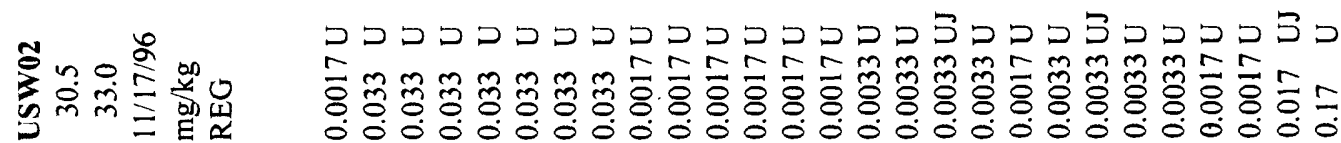

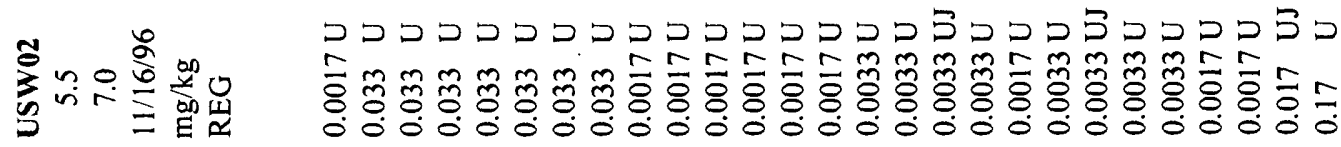

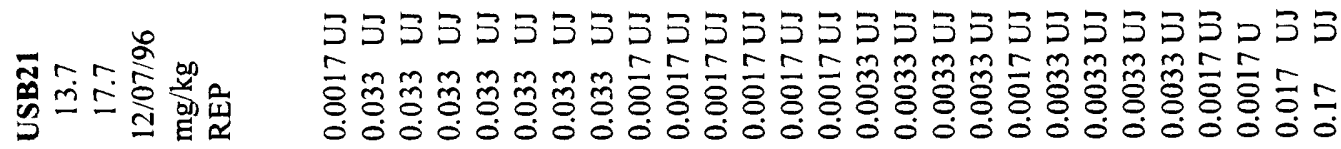

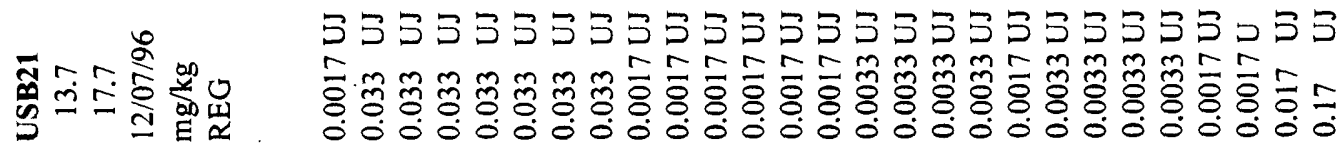

8

范

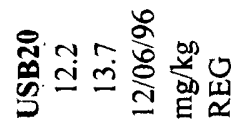

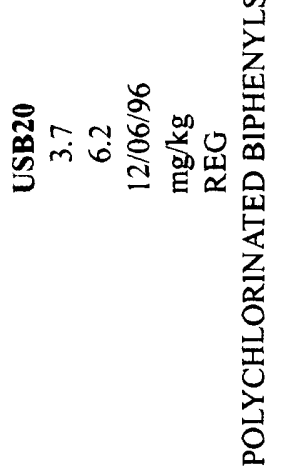

总总

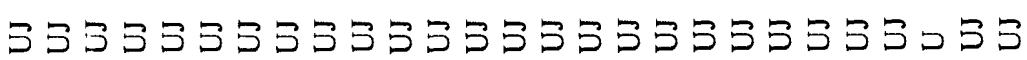

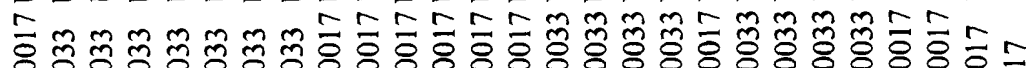

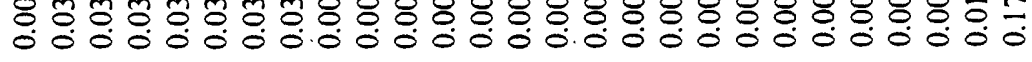

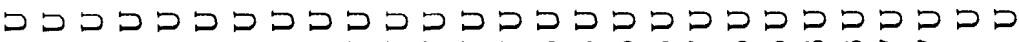

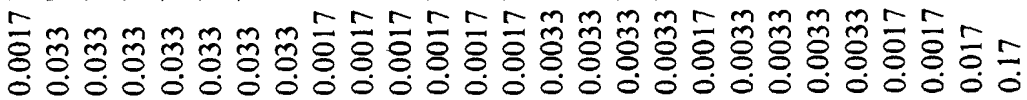

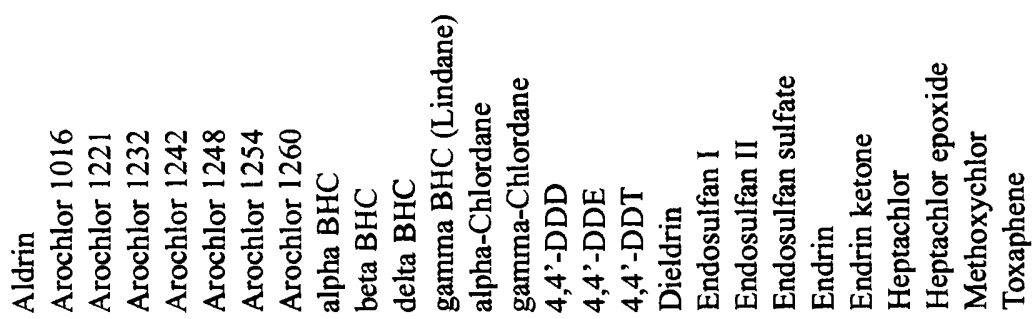


के

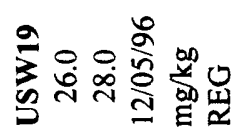

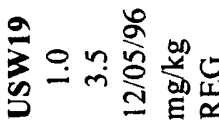

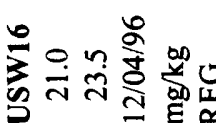

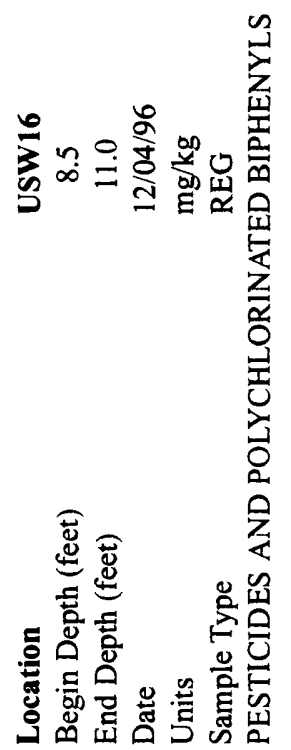

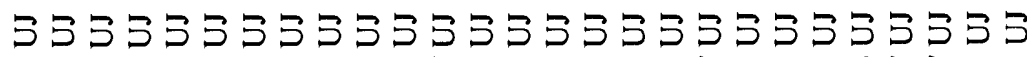
โm

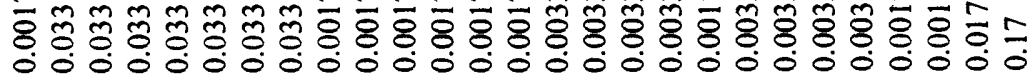

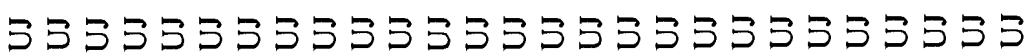

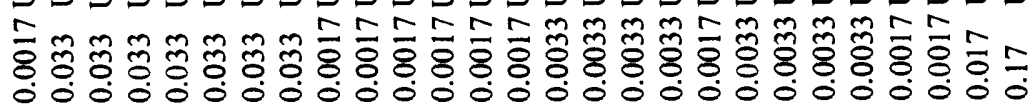

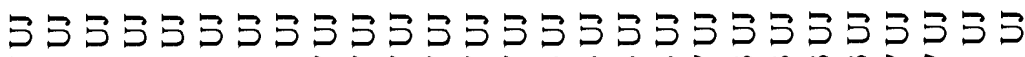

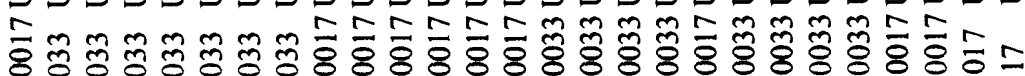
000000000000000000000000000

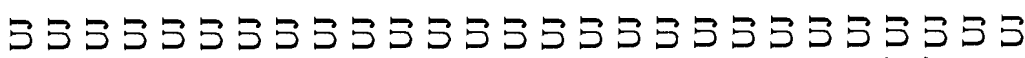

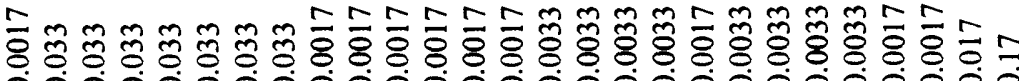

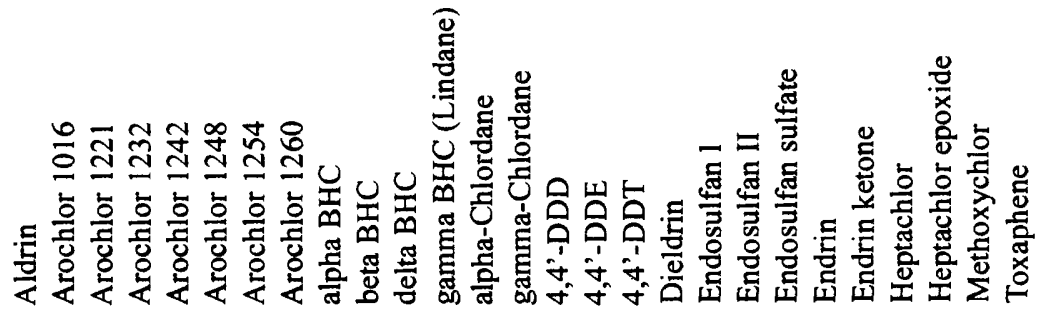




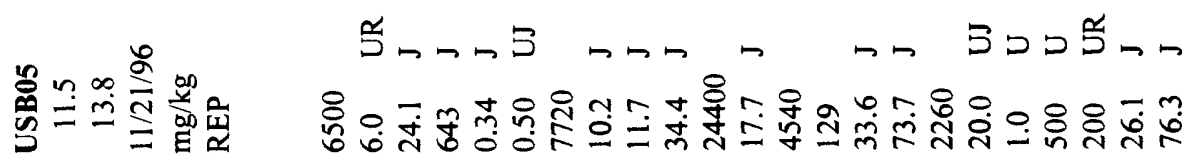

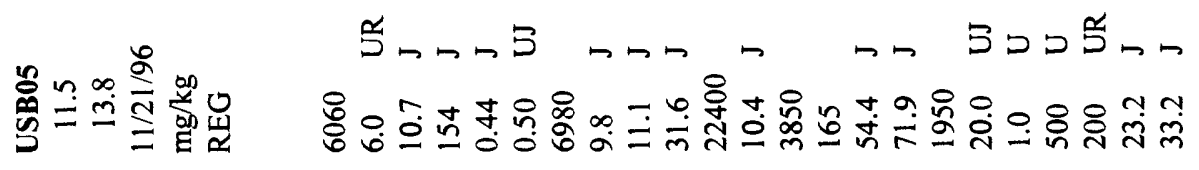

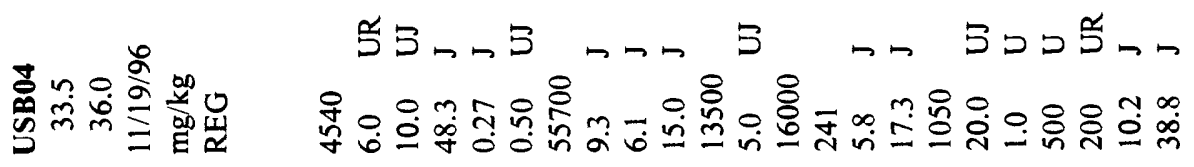

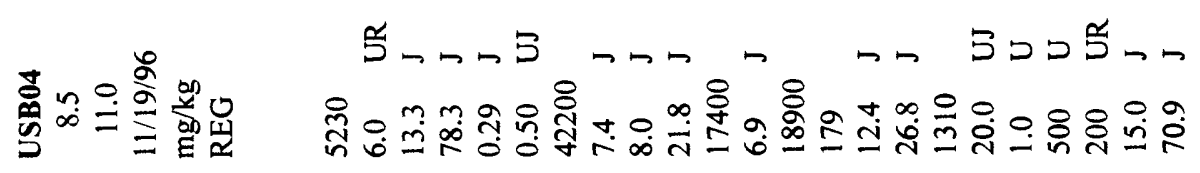

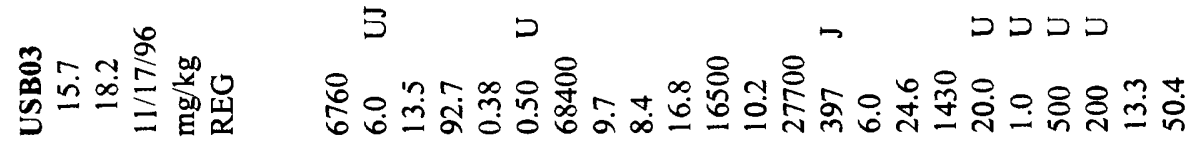

ธิ

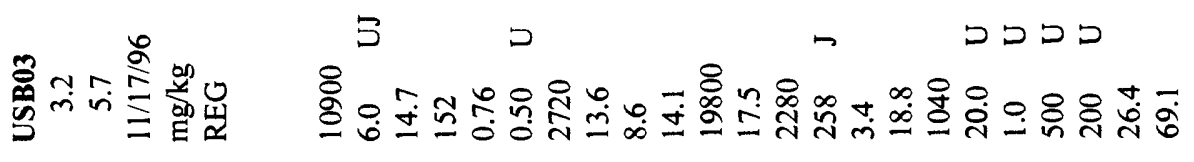

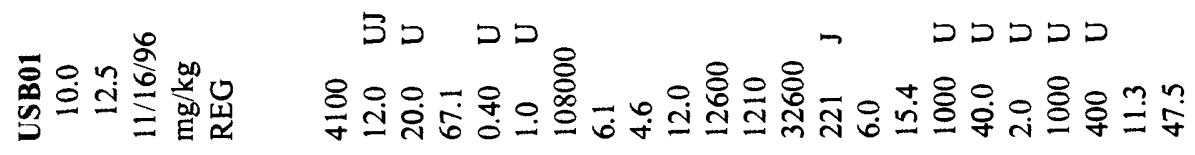

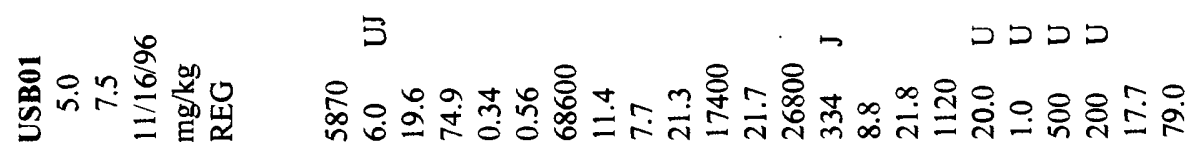

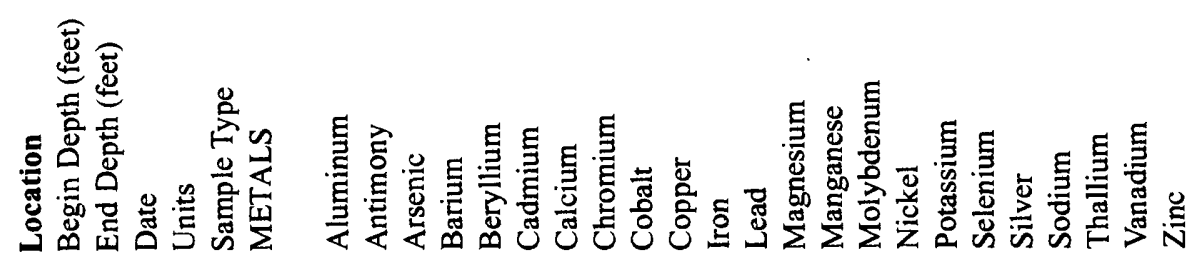




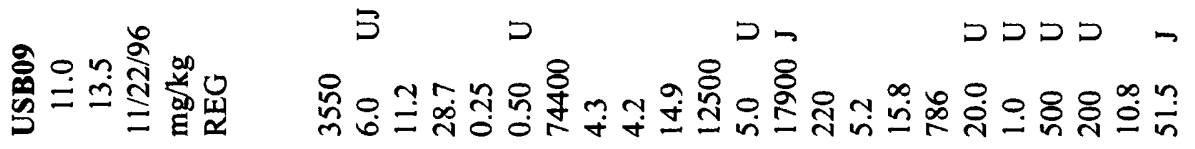

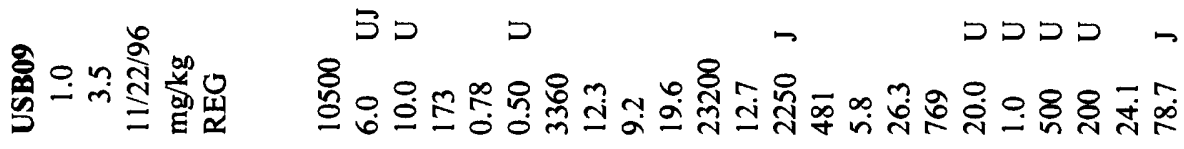

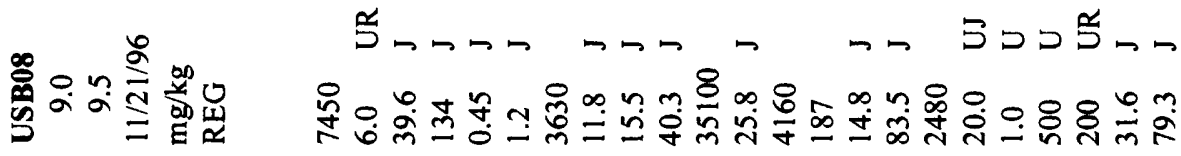

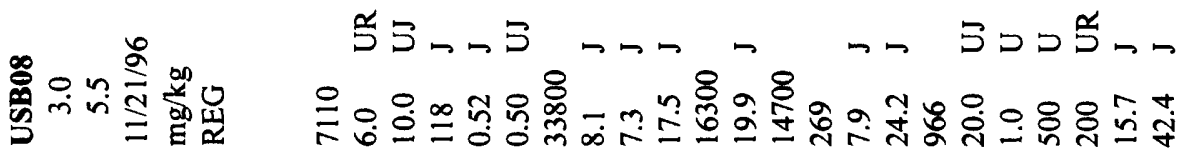

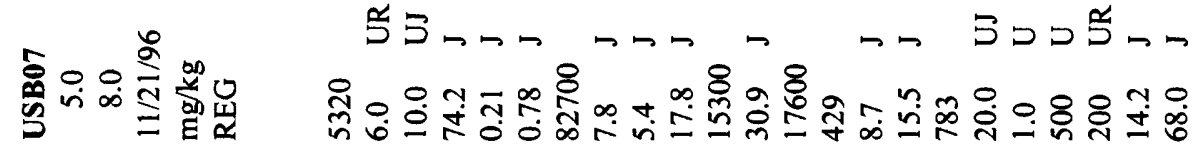

के

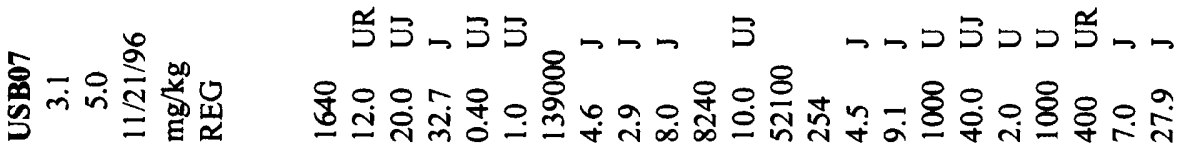

兽象

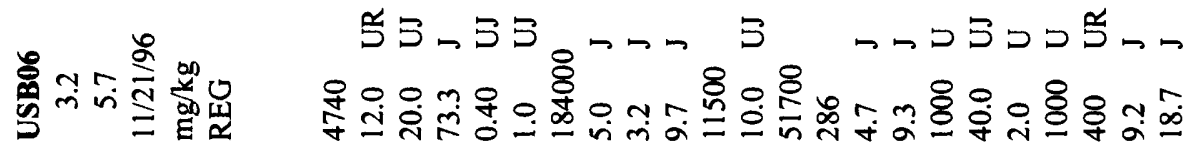

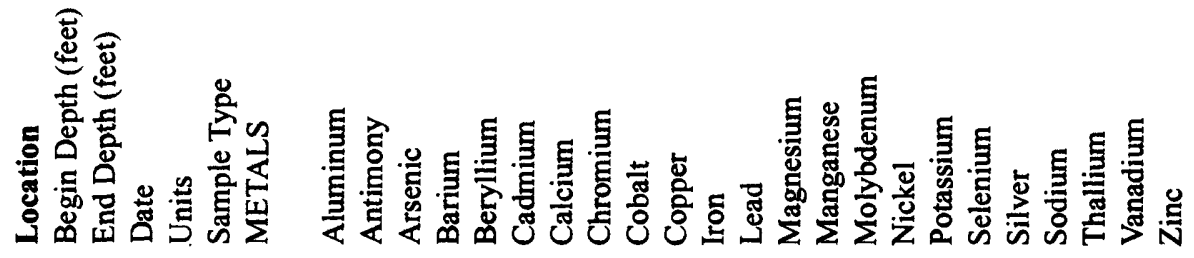




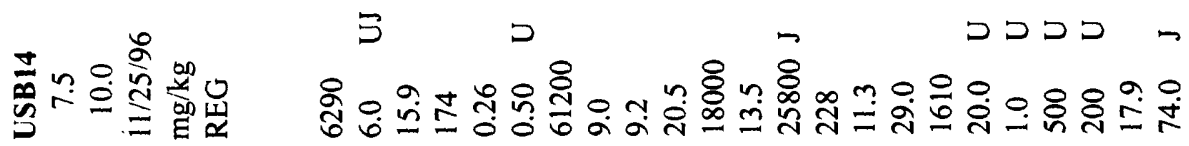

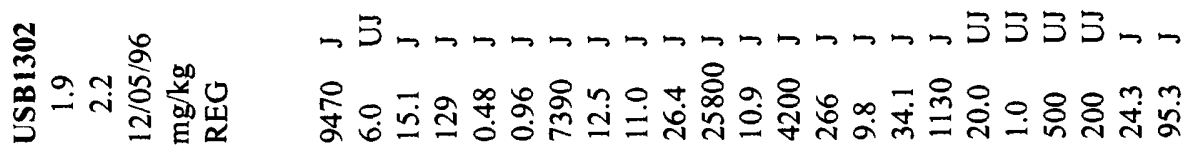

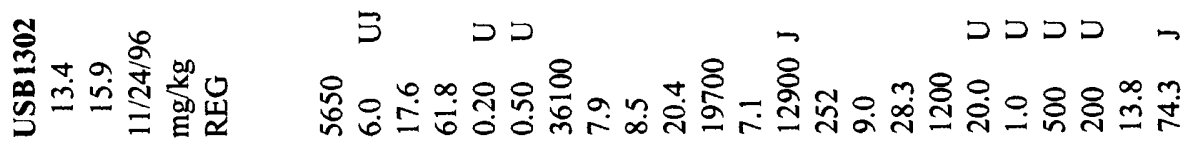

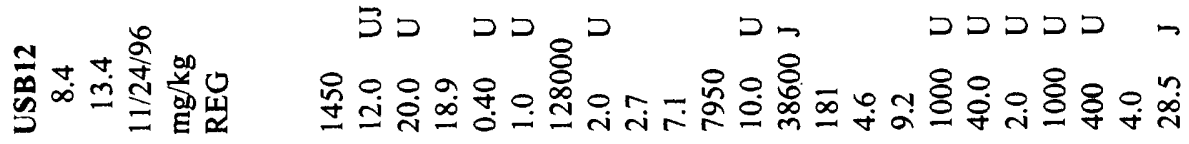

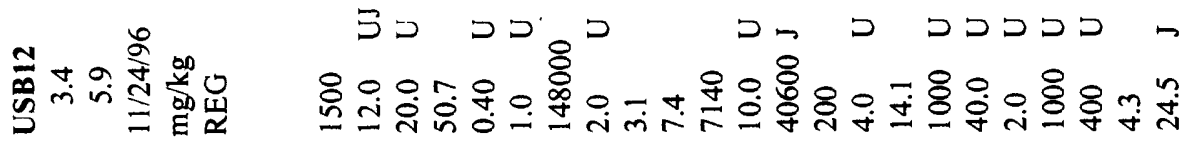

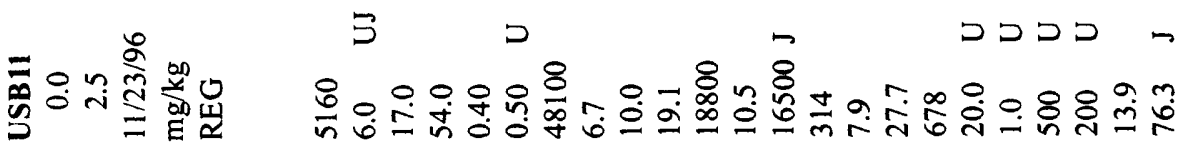

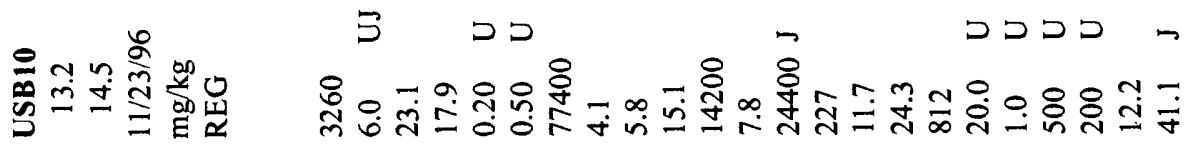

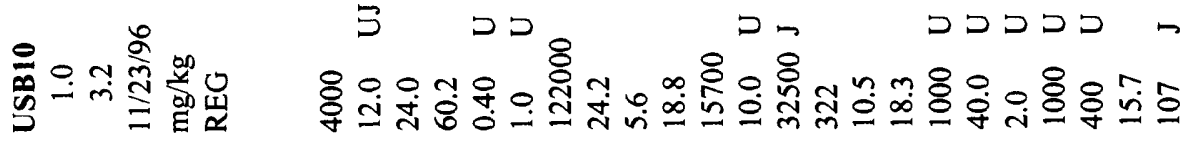

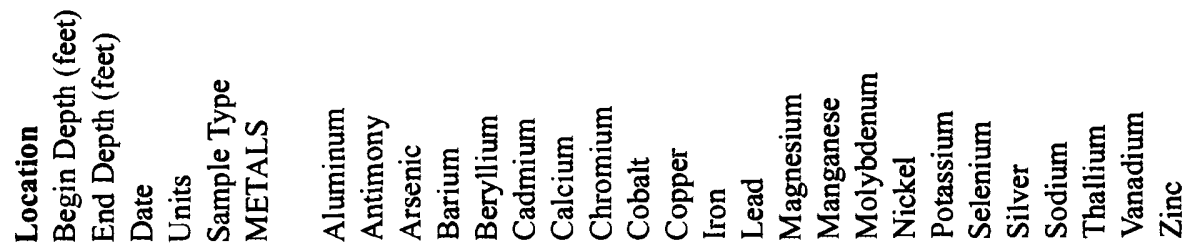




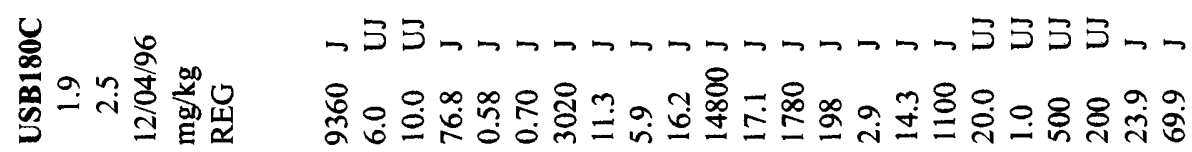

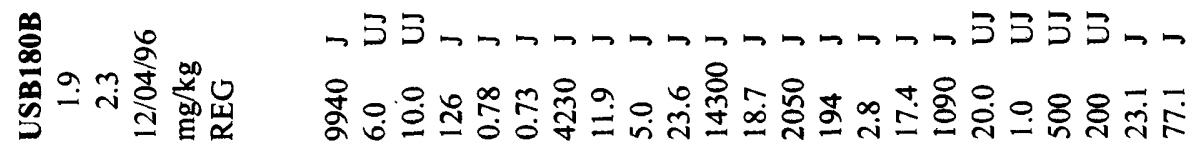

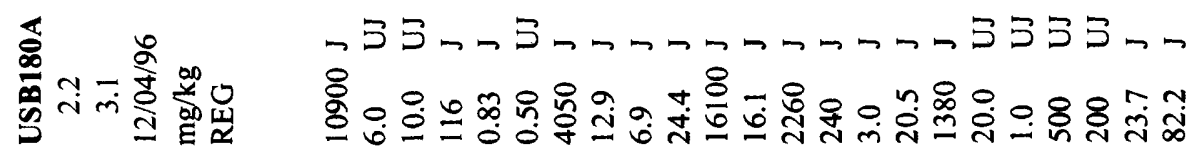

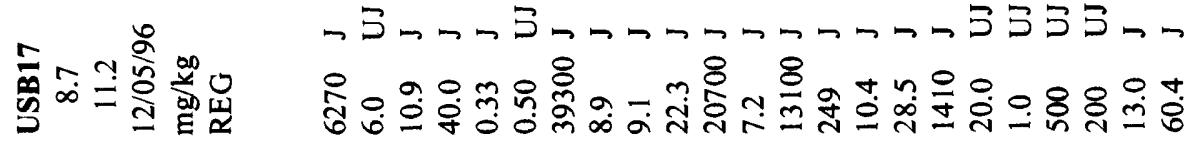

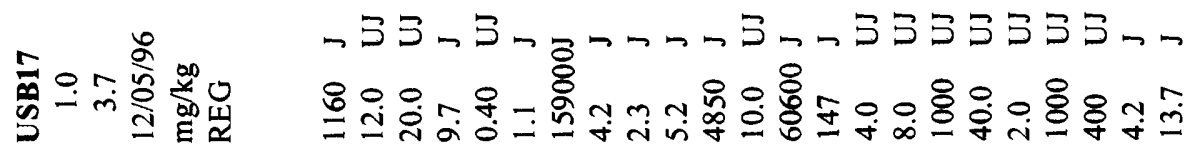

के

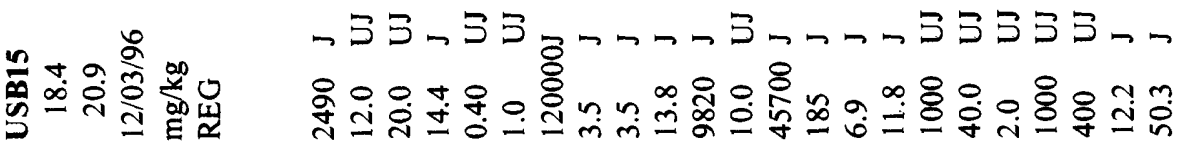

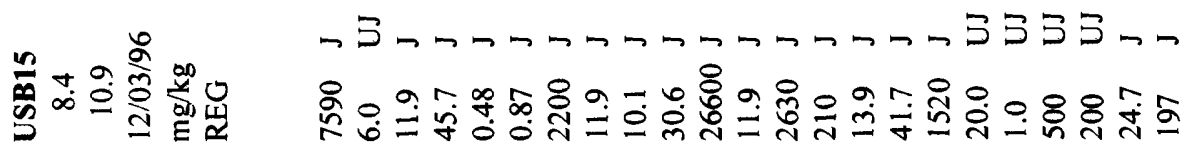

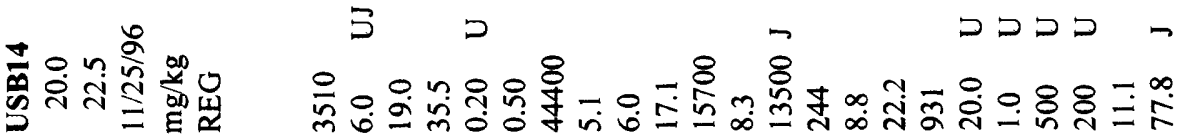

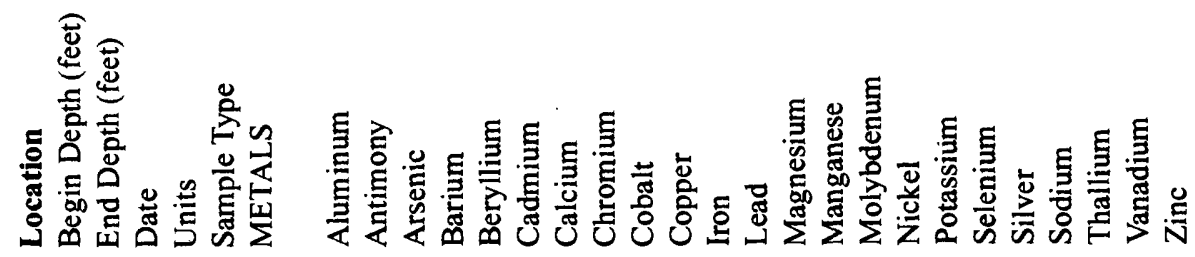




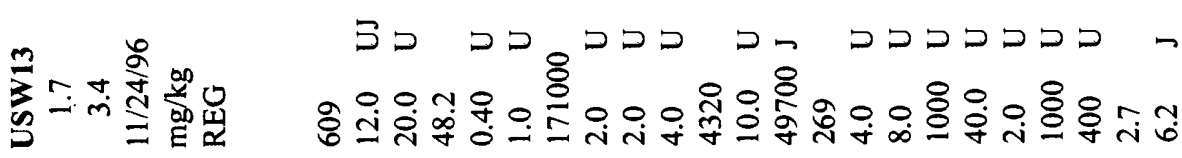

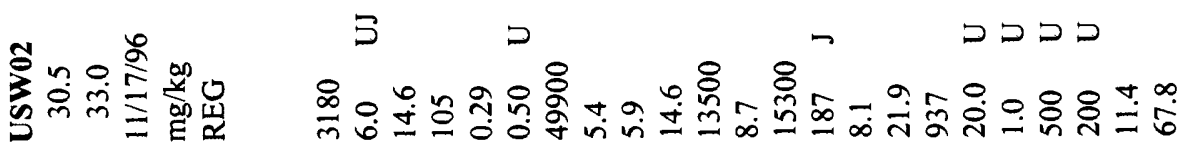

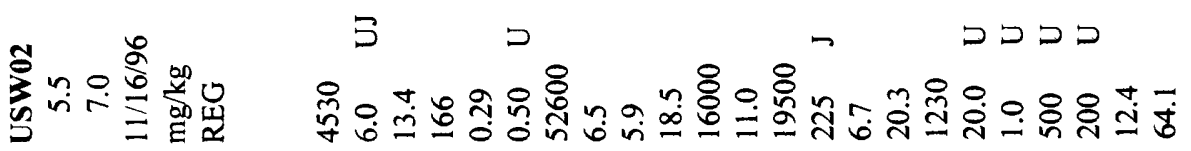

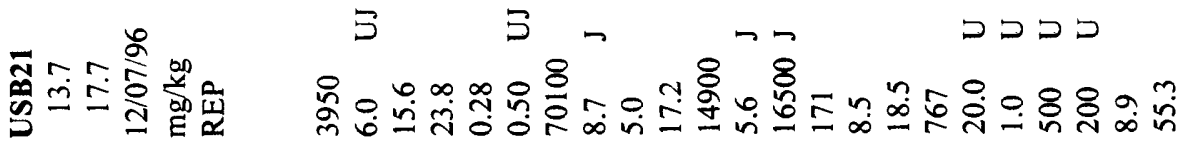

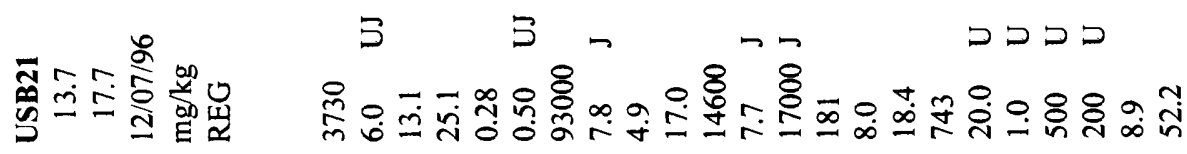

के

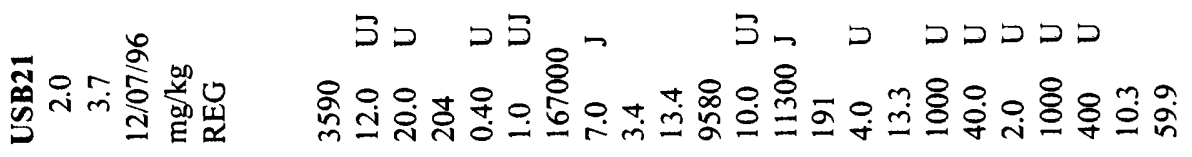

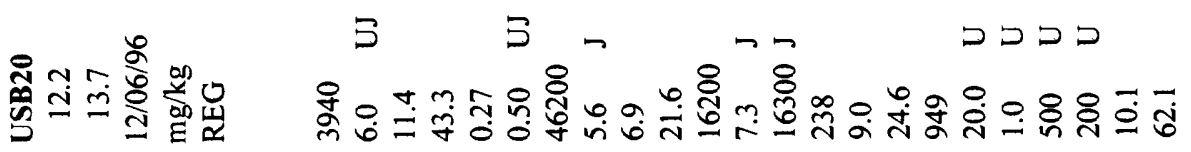

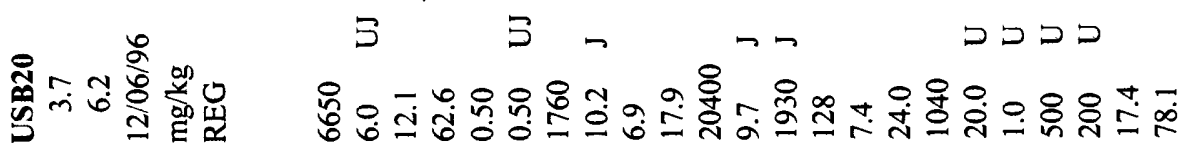

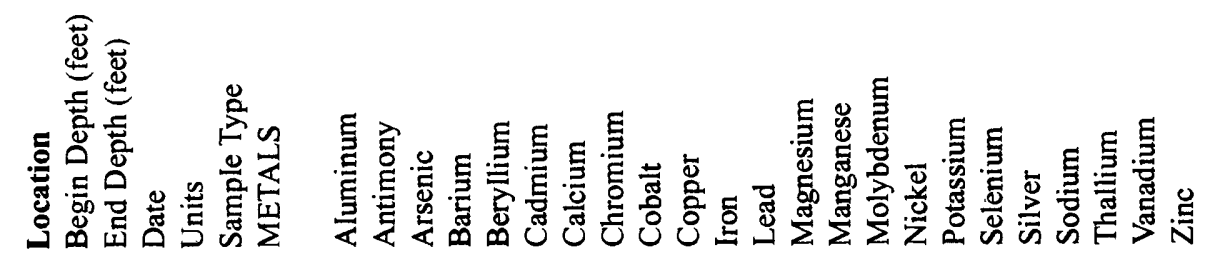




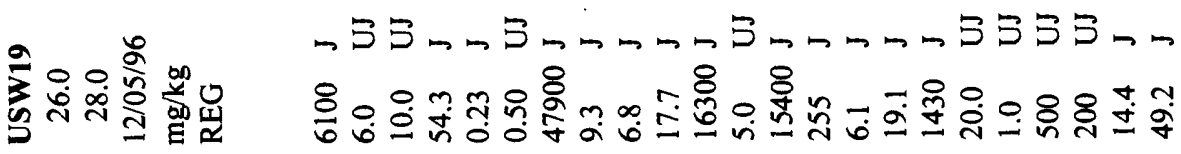

ळे

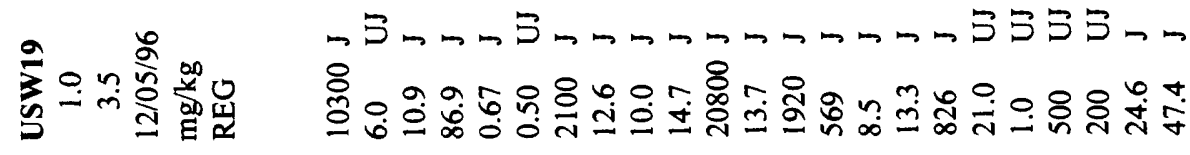

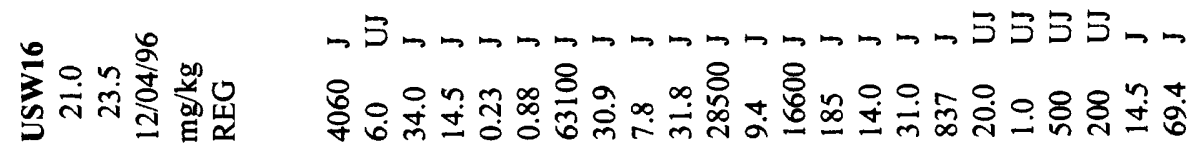

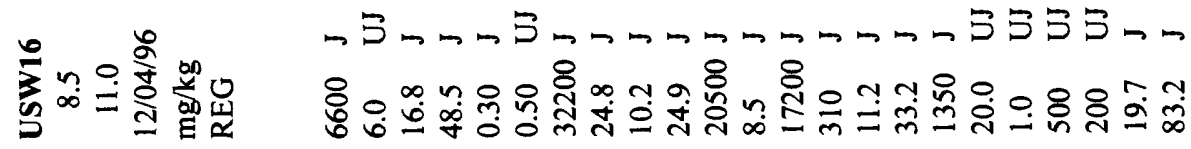

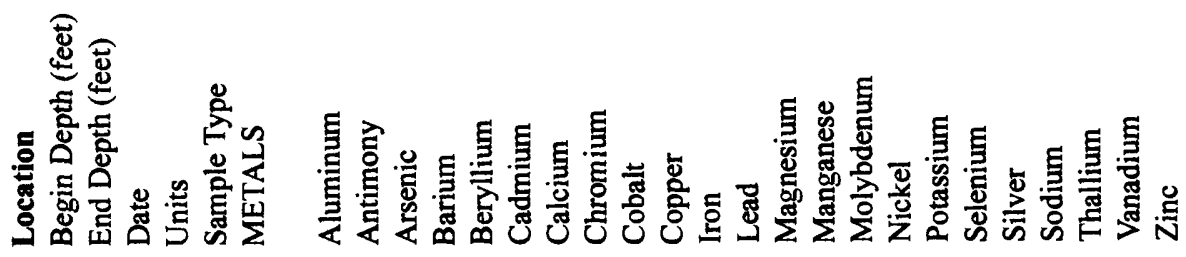




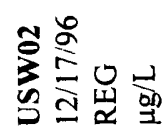

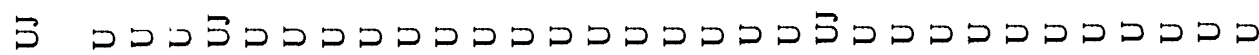

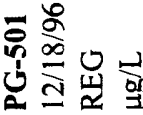

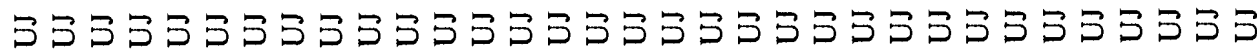

ํํㅇำ

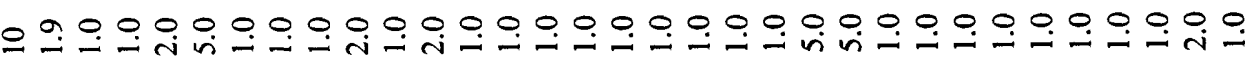

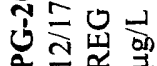

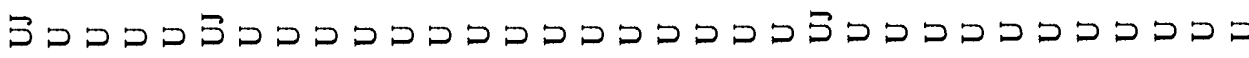

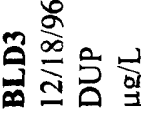

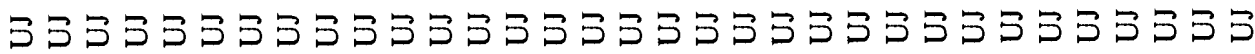

す

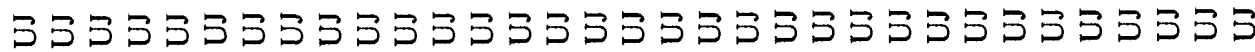

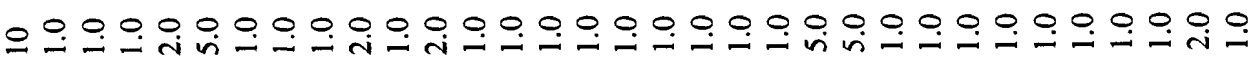

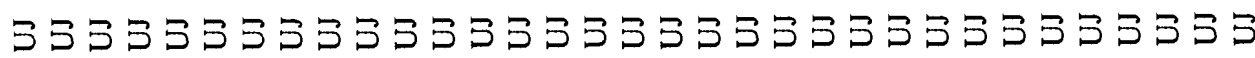
商交出

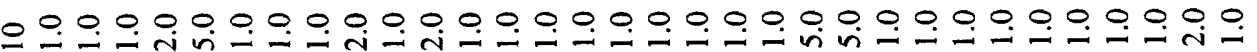

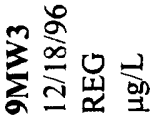

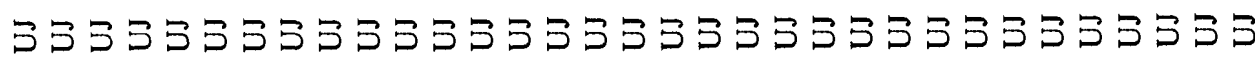
高空总

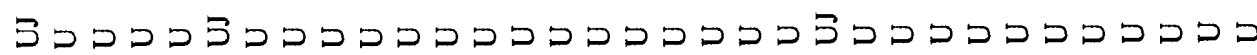

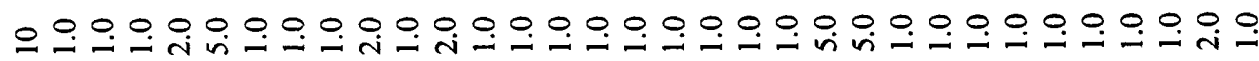

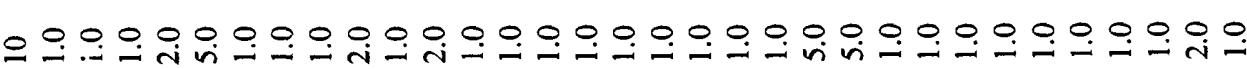

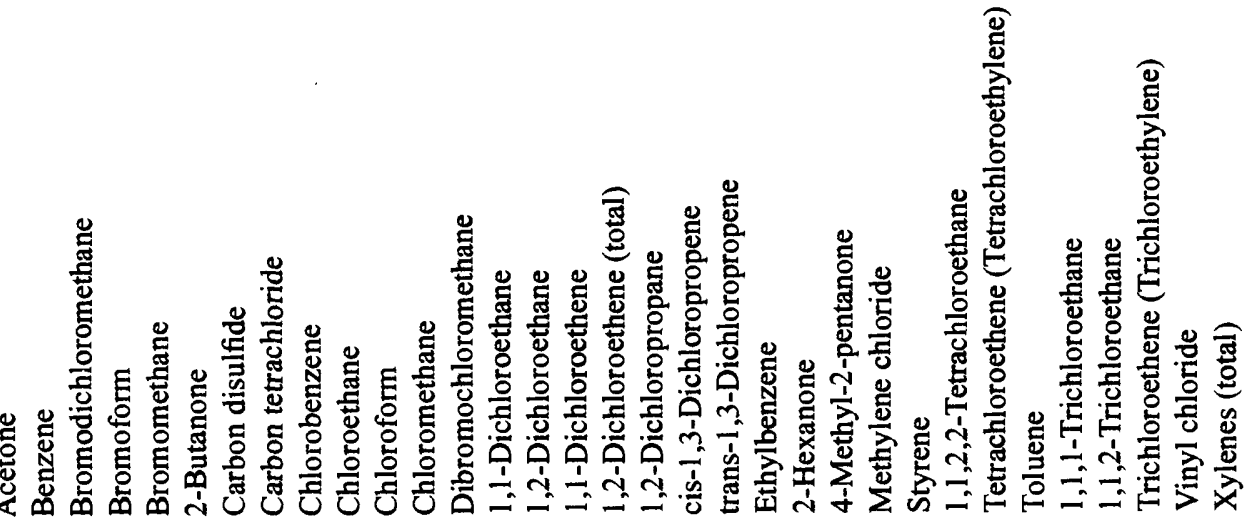




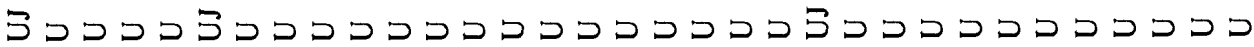

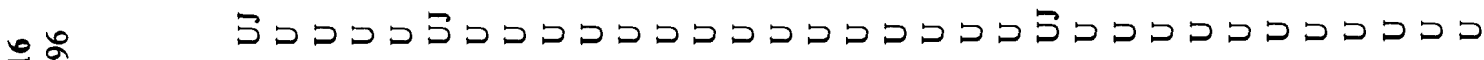

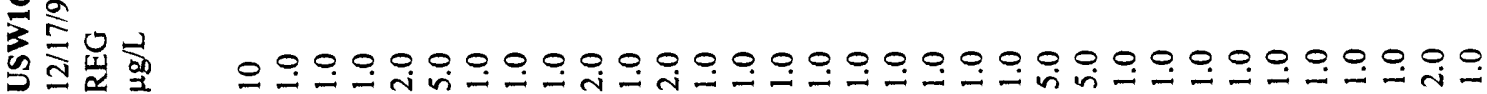

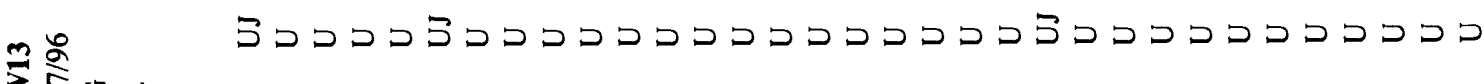

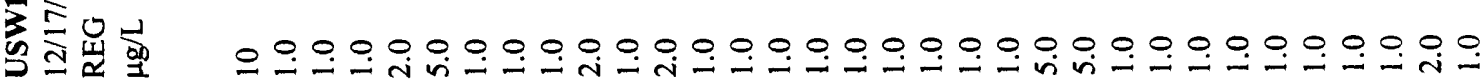

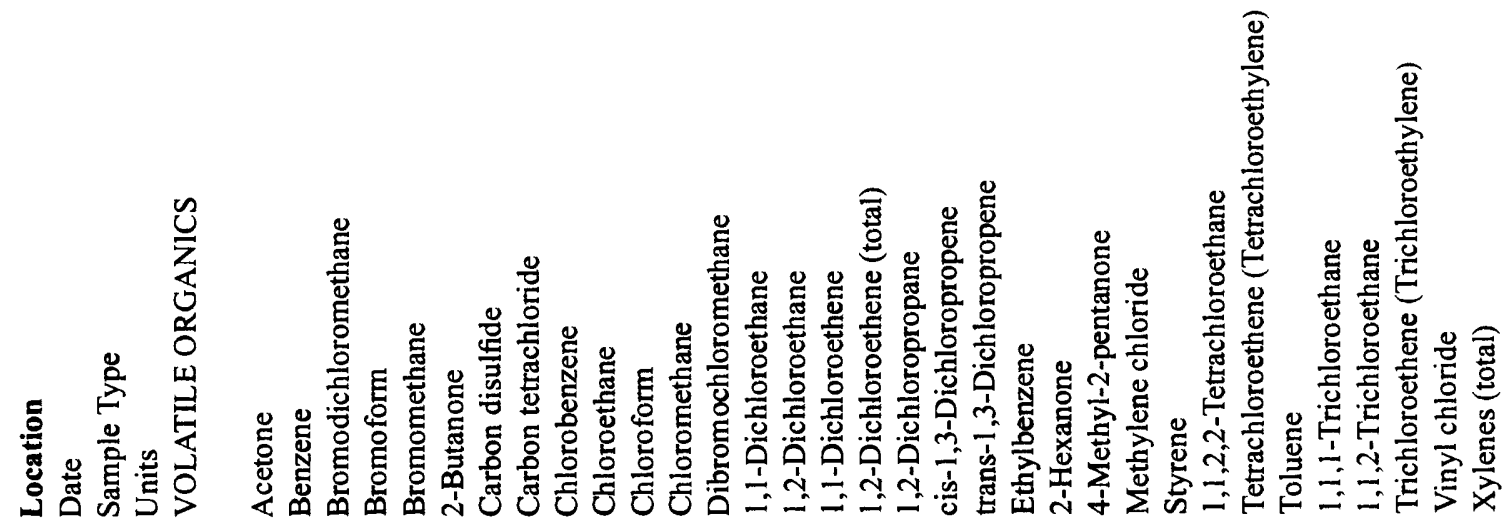




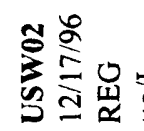

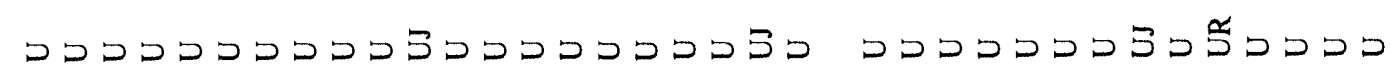

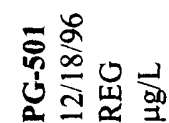

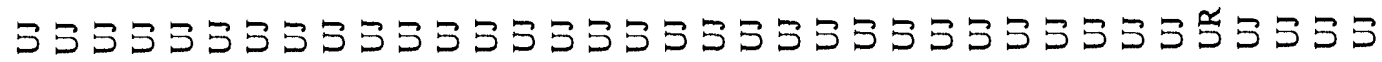

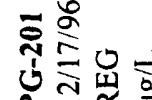

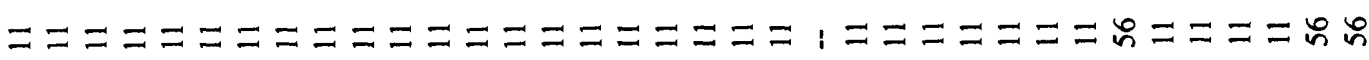

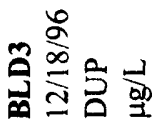

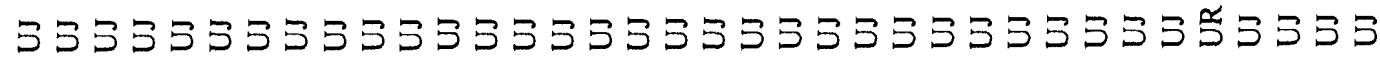

\

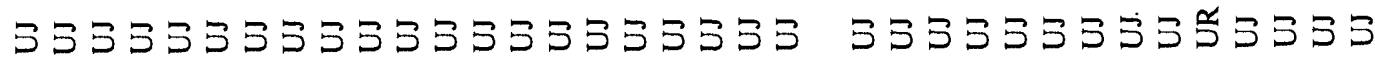

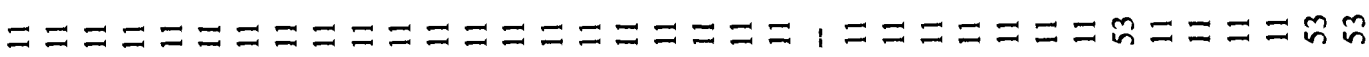

융

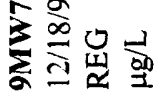

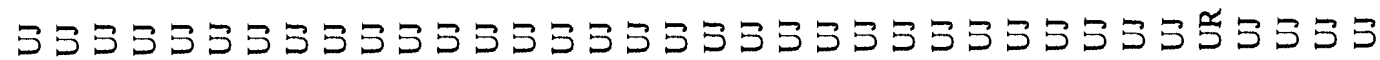

으으으으으으으으으으으으으으으으으으으으으으으으으츄으으으대츄

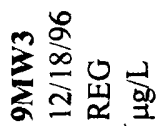

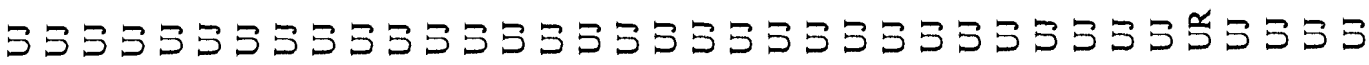
으으으으으으으으으으으으으으으으으으으으으으으으으으으육으으으유규

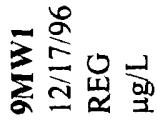

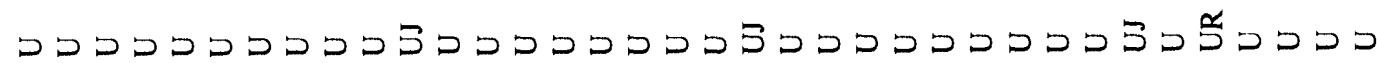

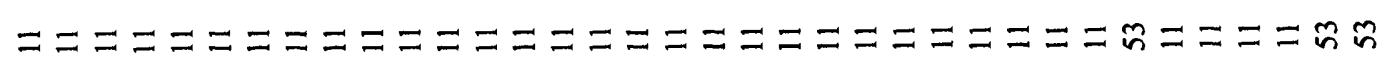
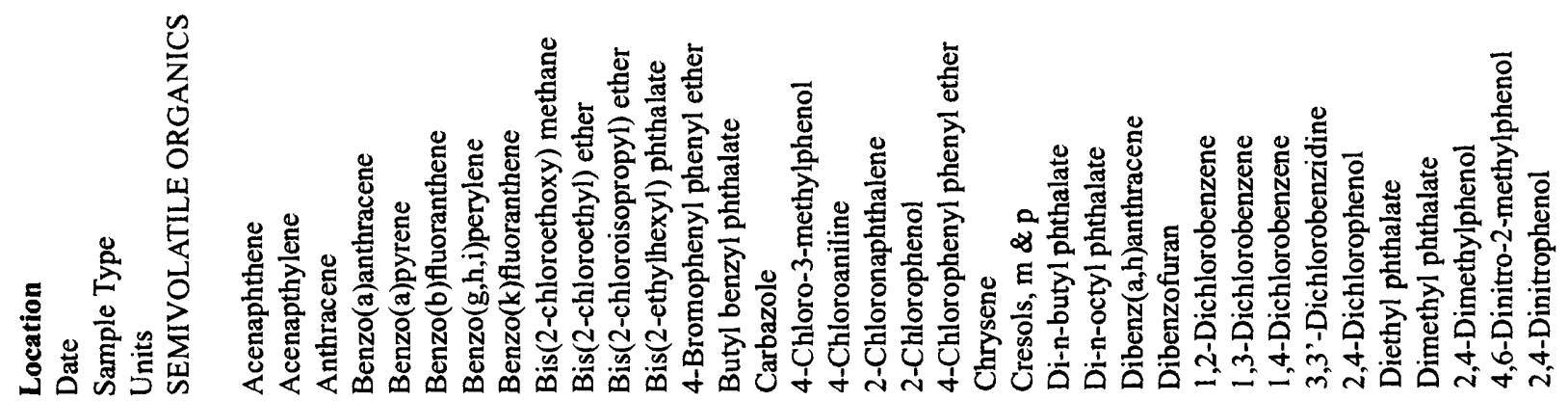


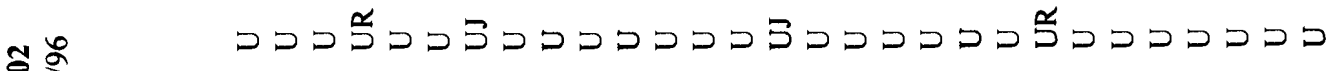

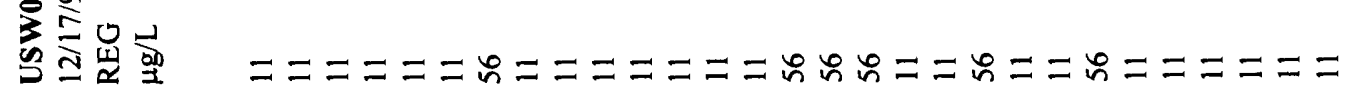
ー

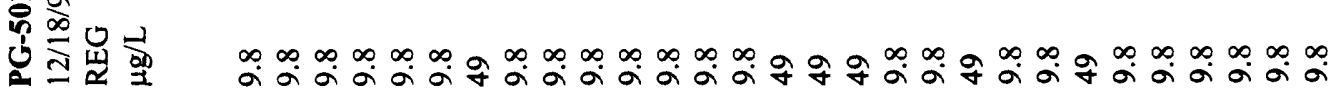

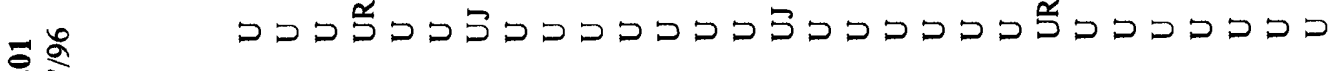

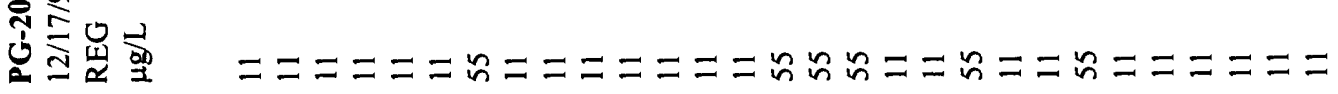

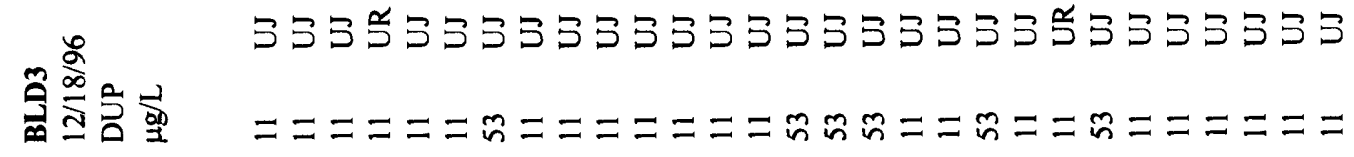

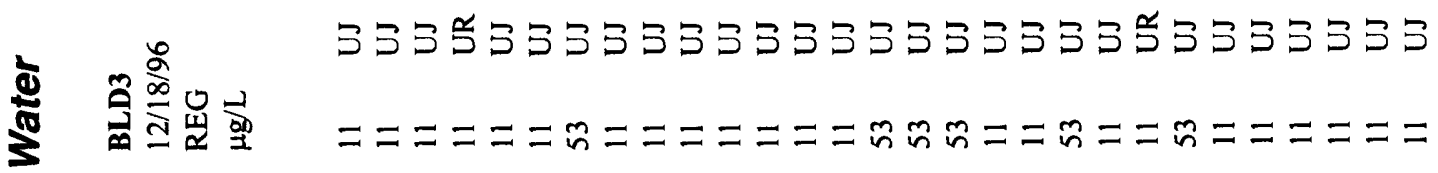
కั่

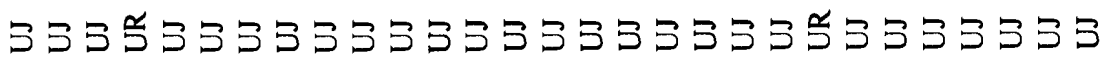

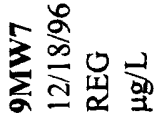

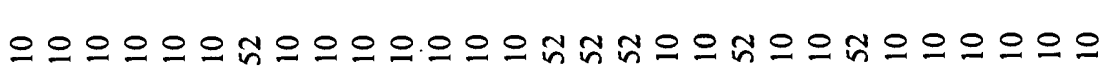

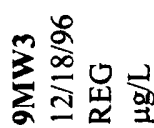
タララ

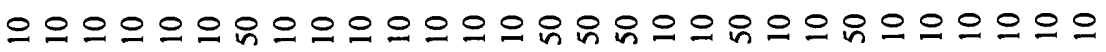

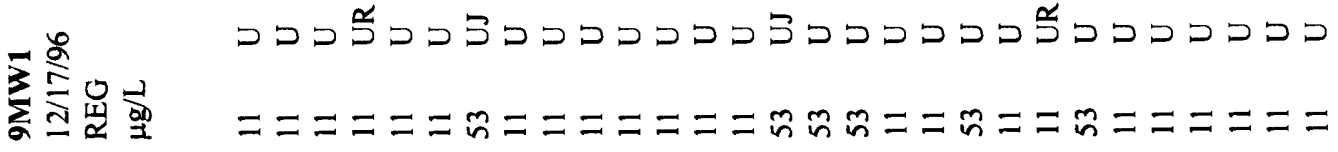

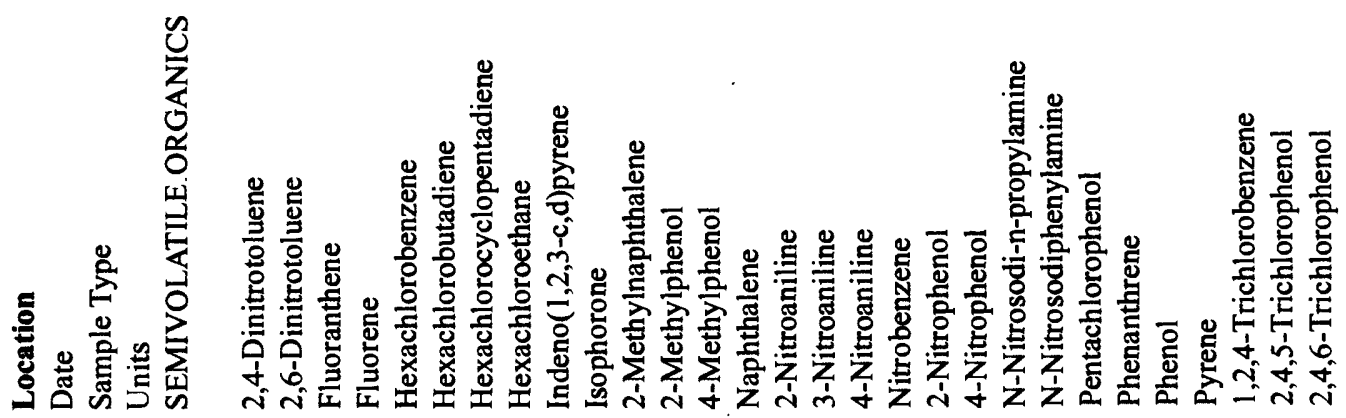


ดั

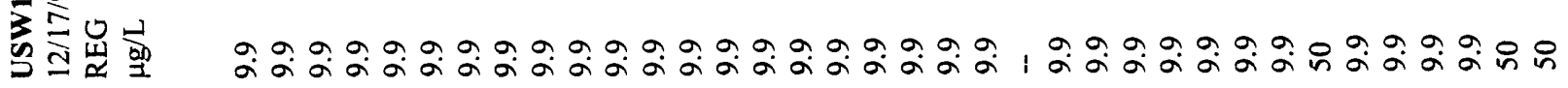

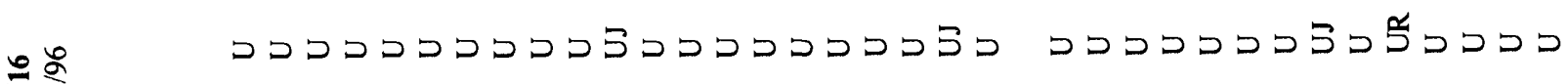

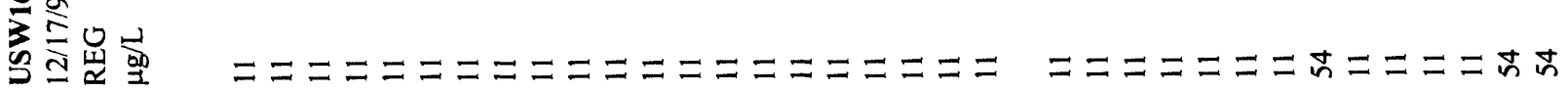

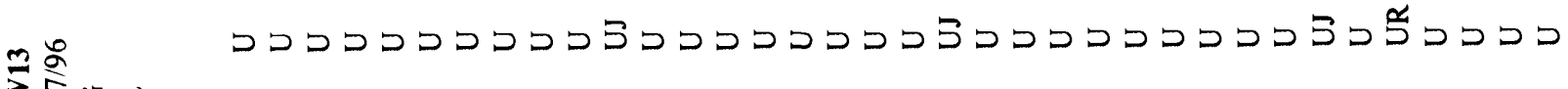

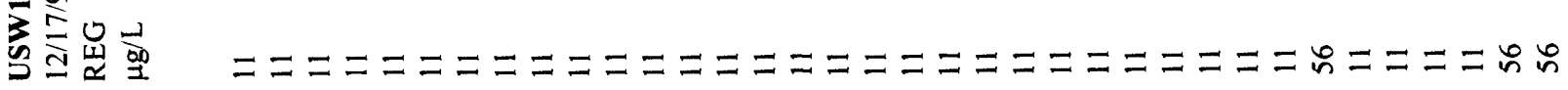

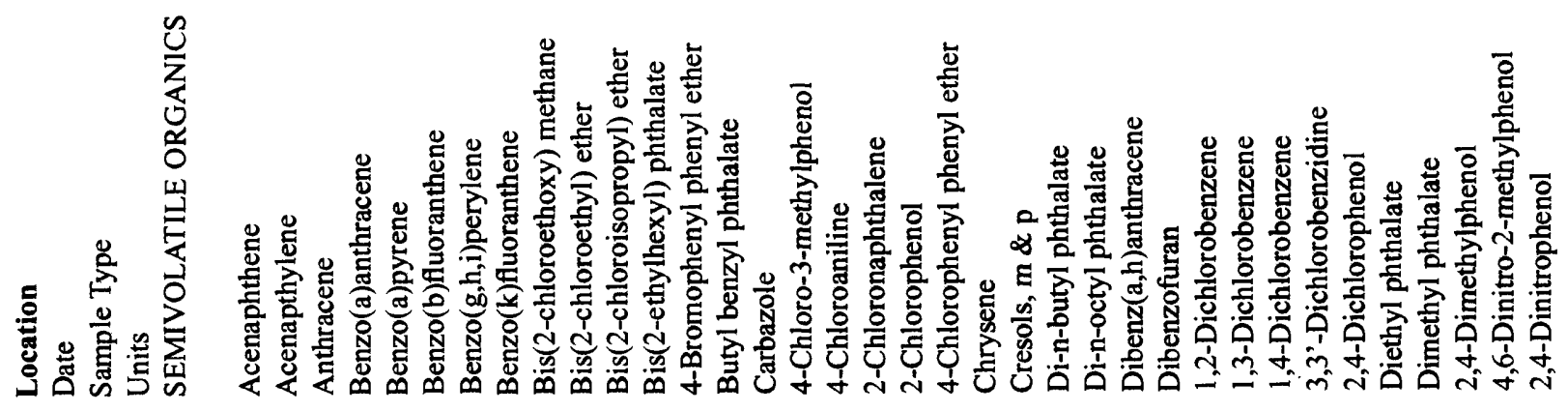




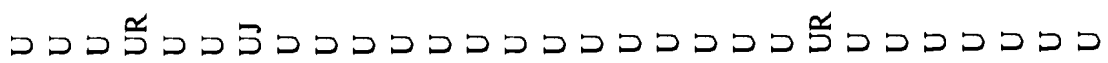
ล่ ส่

ํํำำㄴํำ

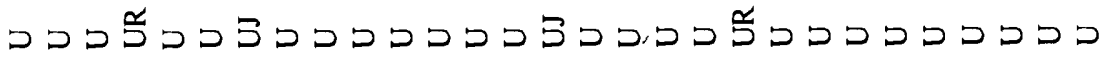

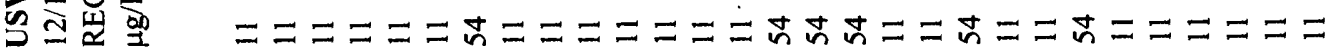

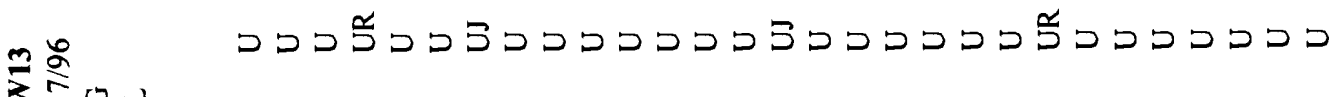

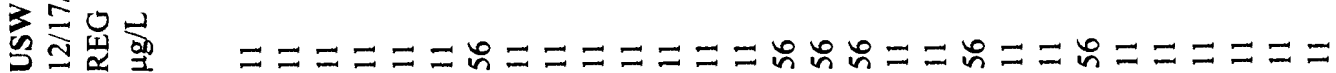

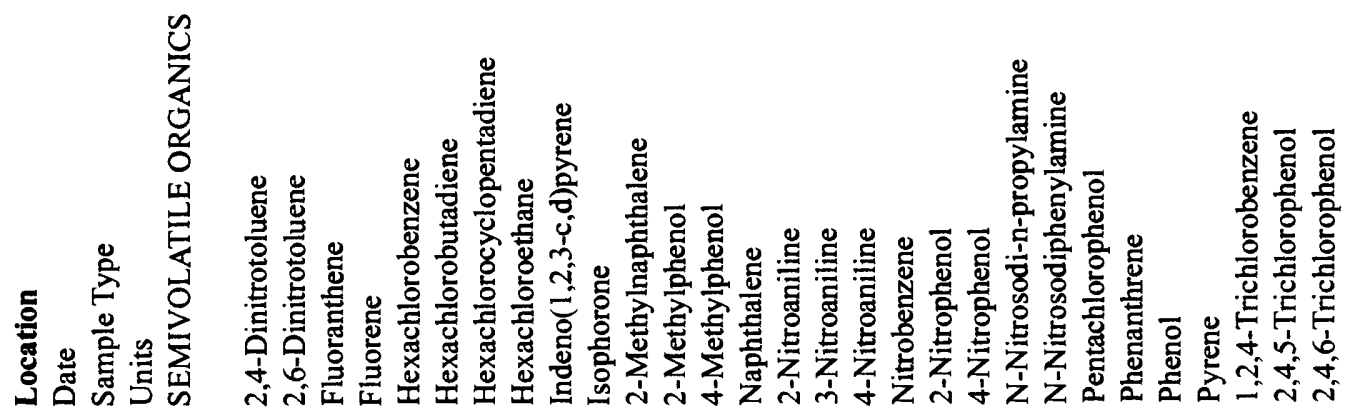




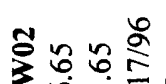

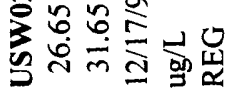

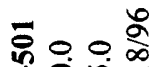

לุ⿺辶่

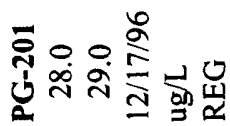

:

के

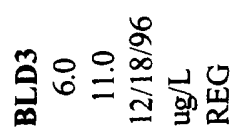

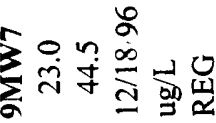

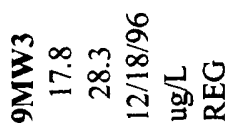

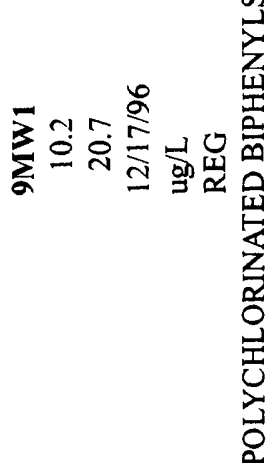

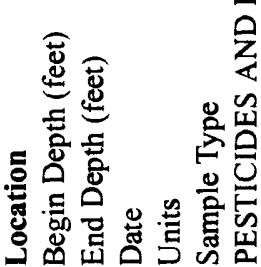

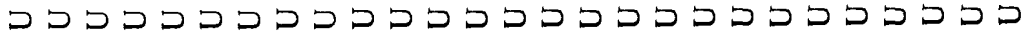

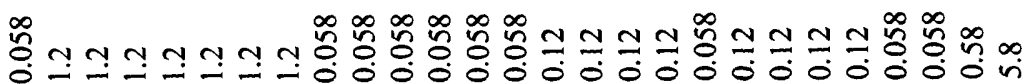

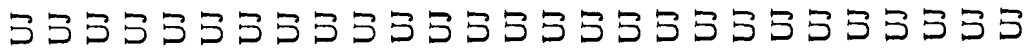

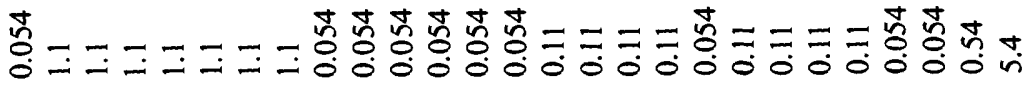

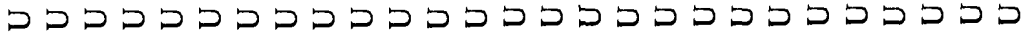

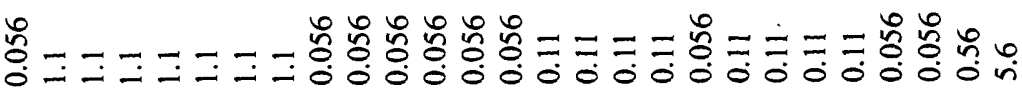

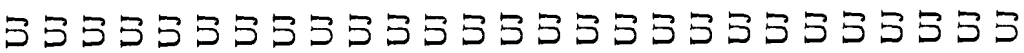

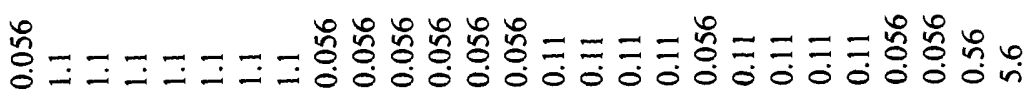

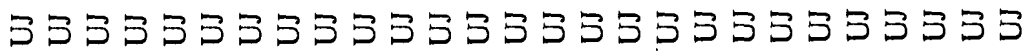

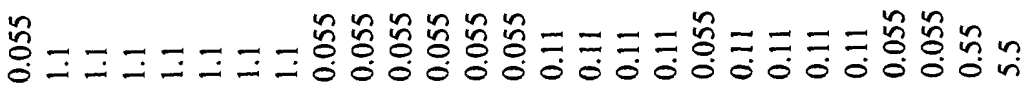

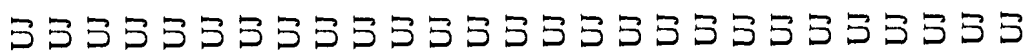

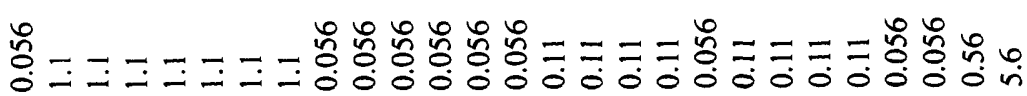

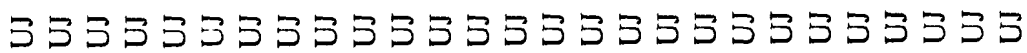

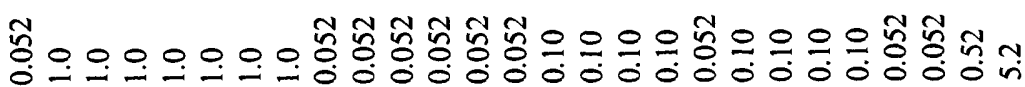

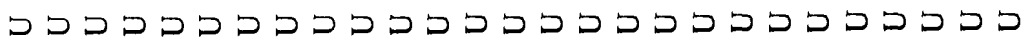

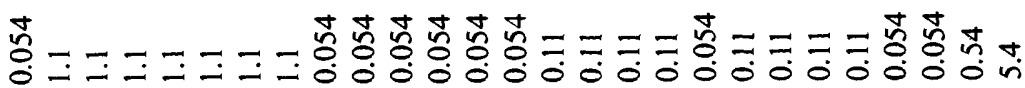

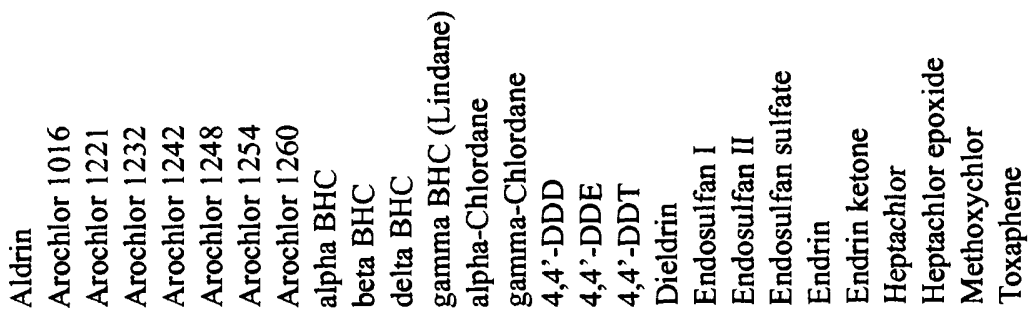




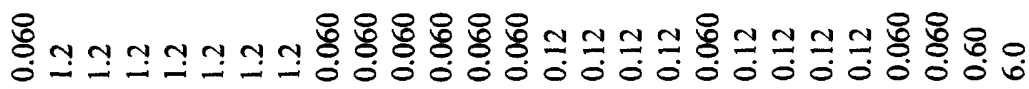




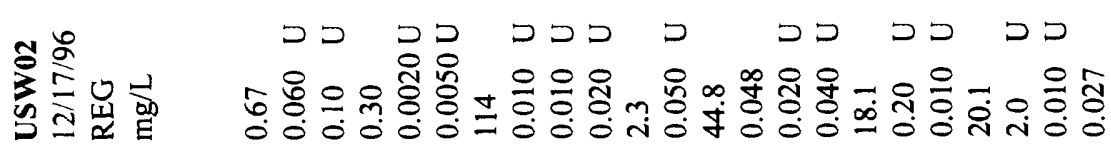

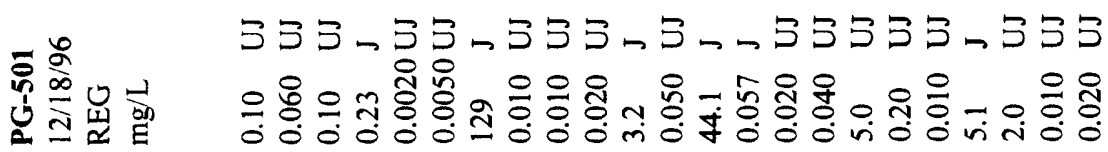

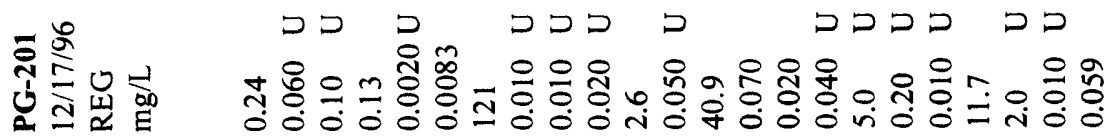

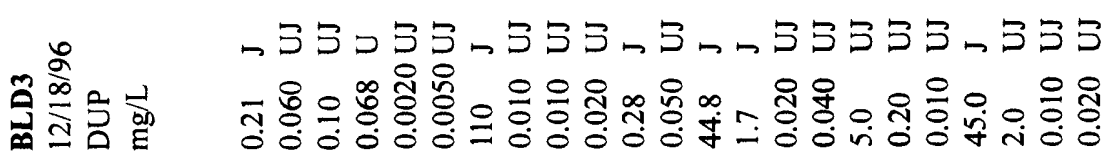

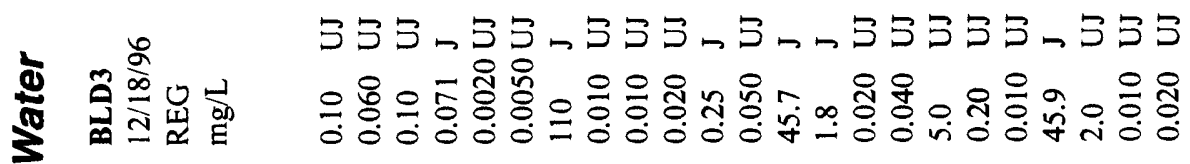

ڤัँ

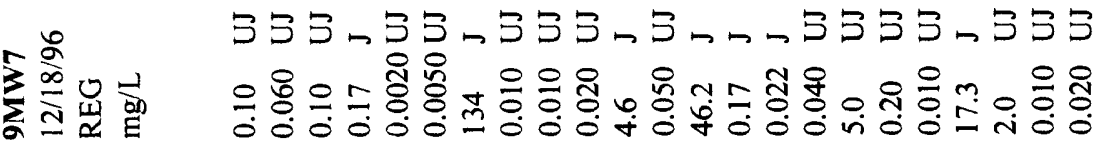

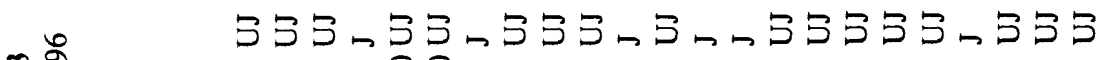

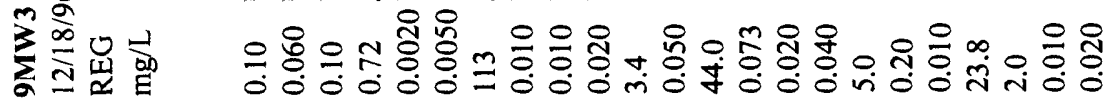

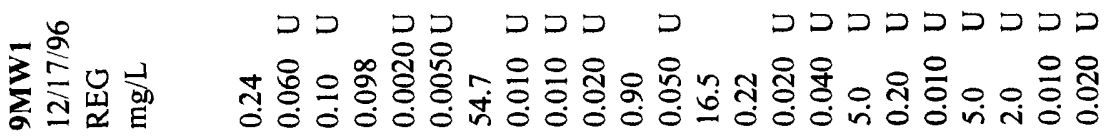

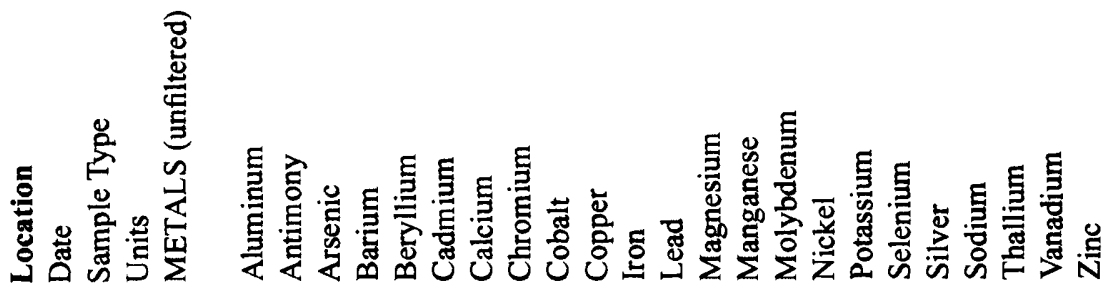




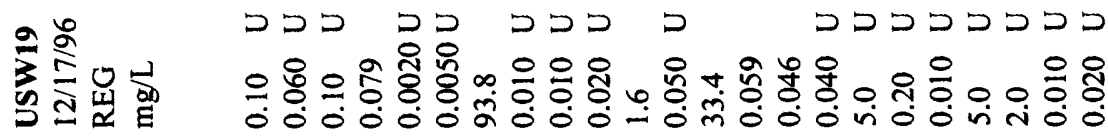

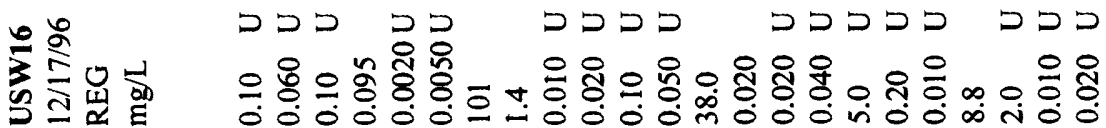

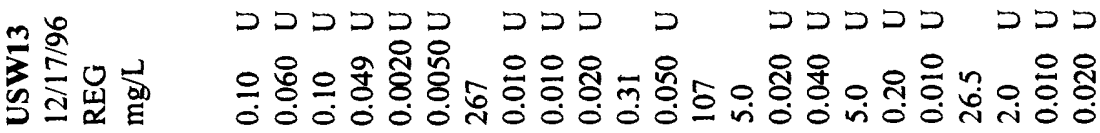

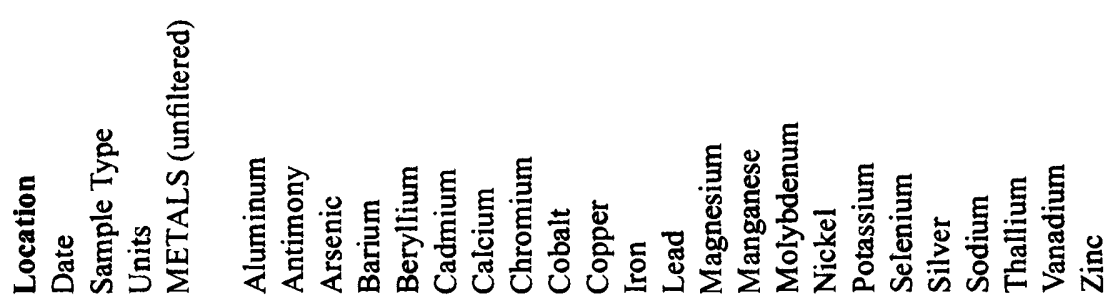




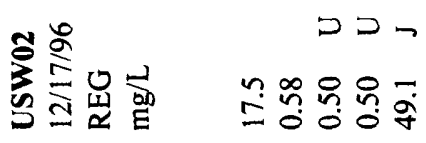

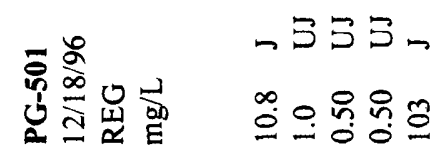

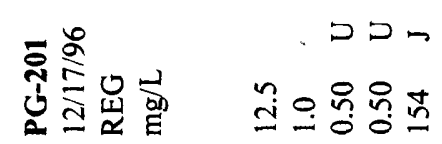

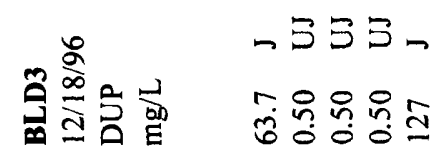

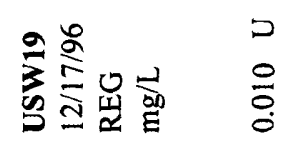

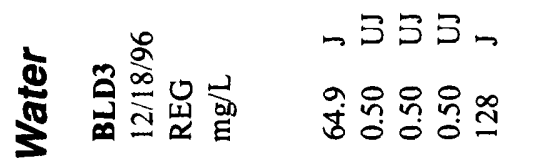

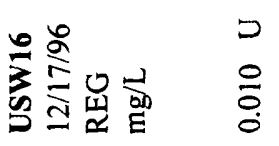

के

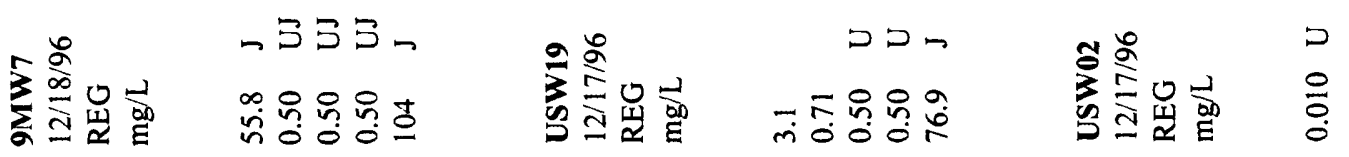

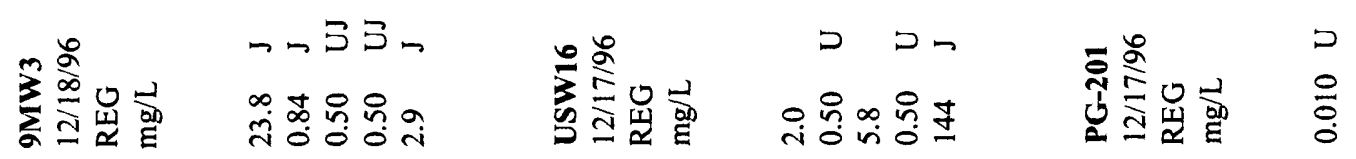

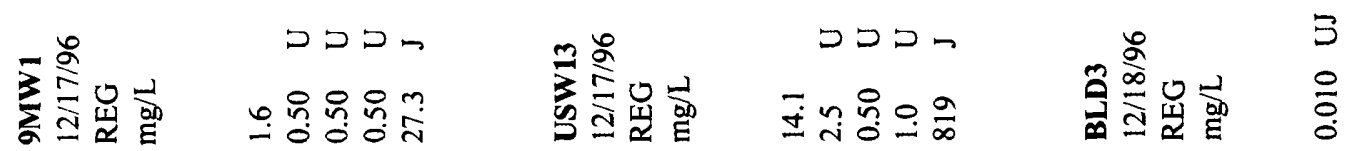

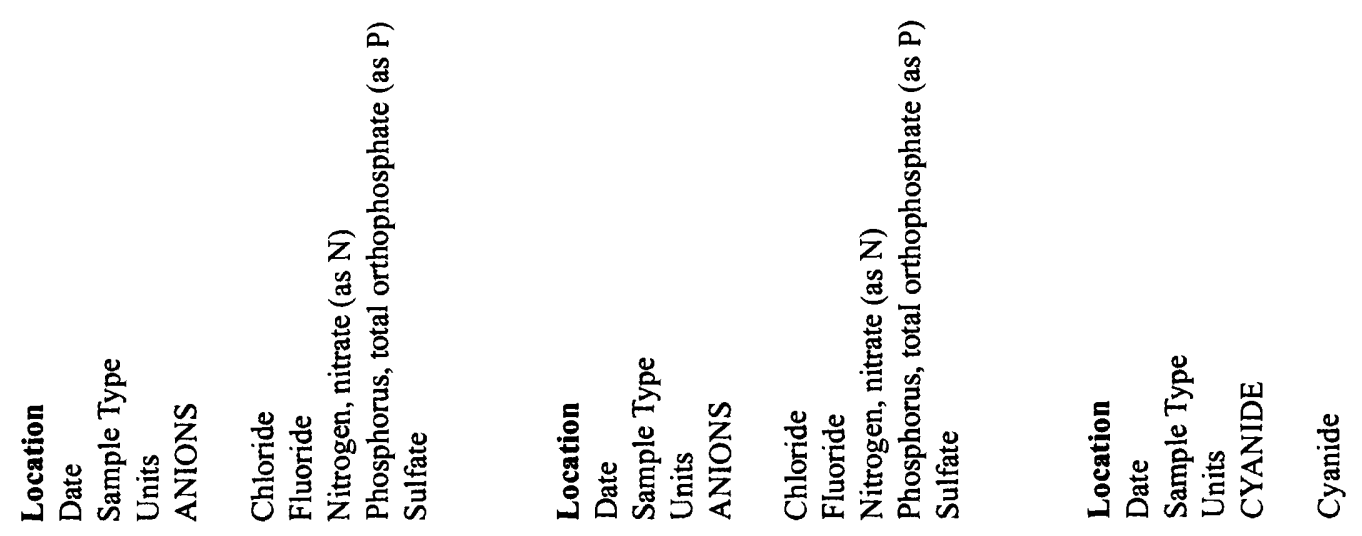




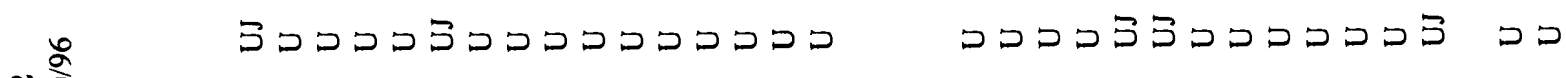

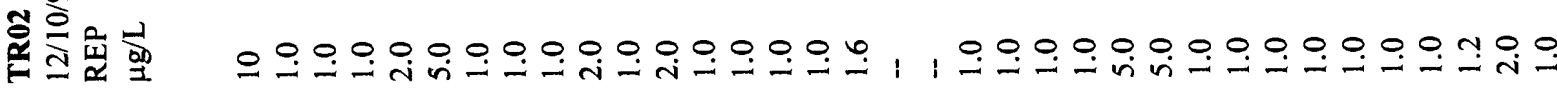

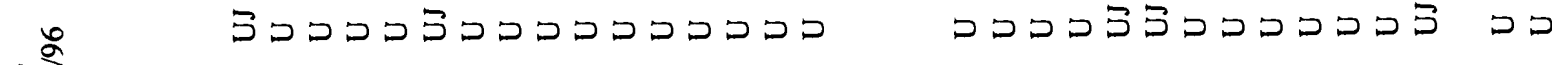

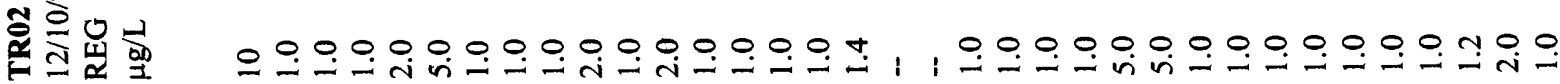

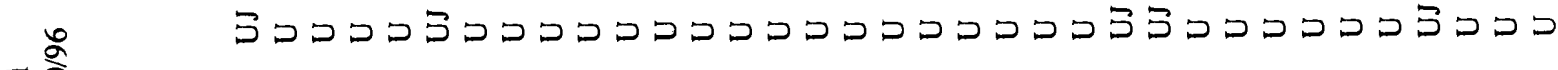

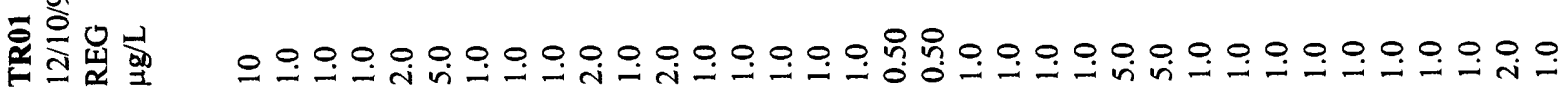

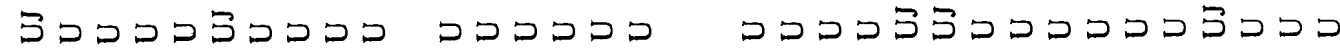

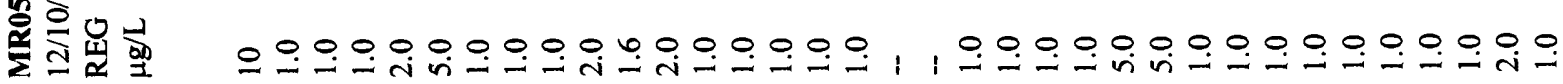

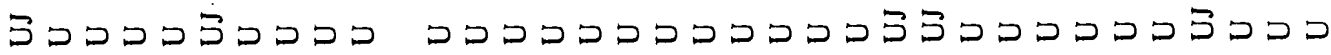

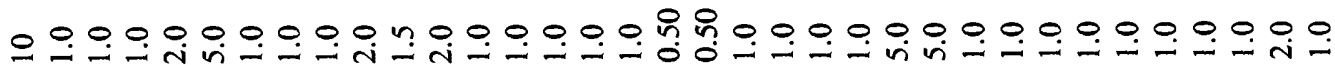

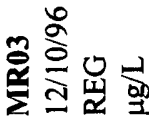

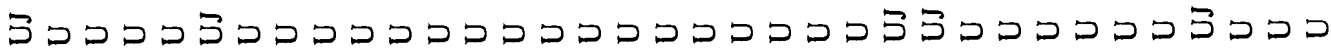

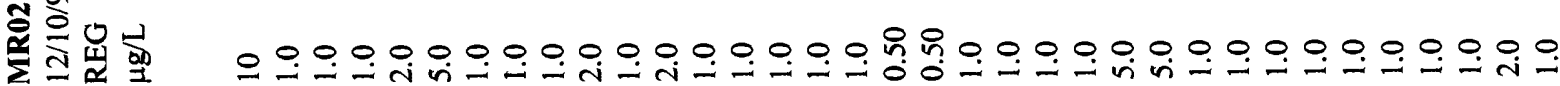

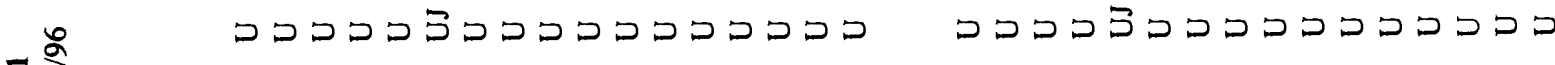

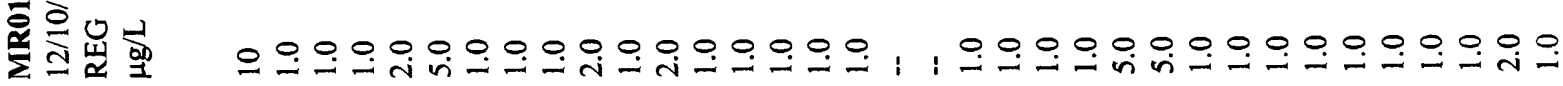

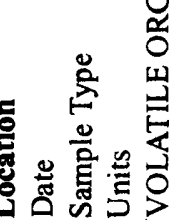
垈
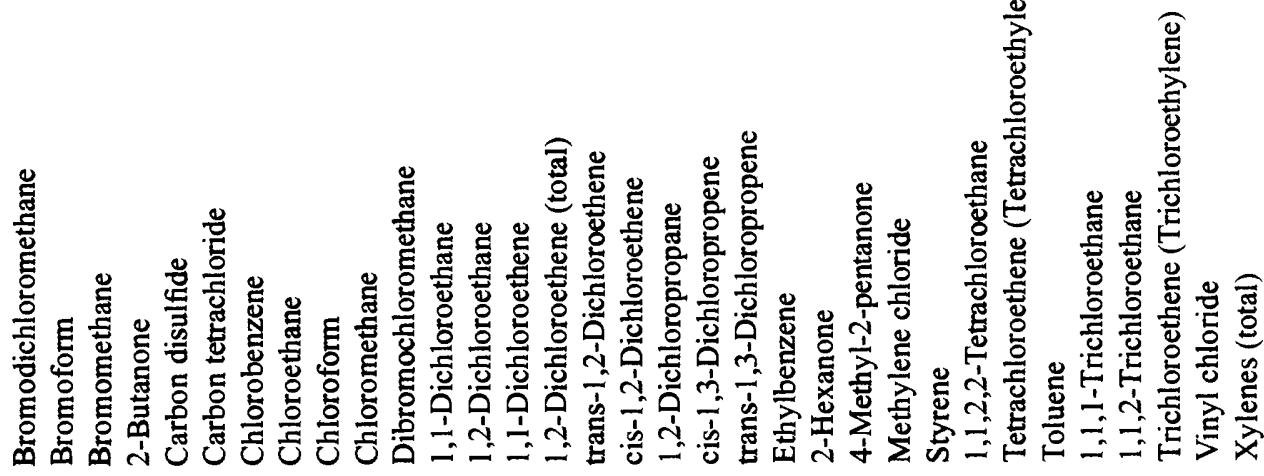


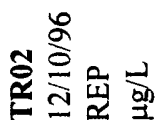

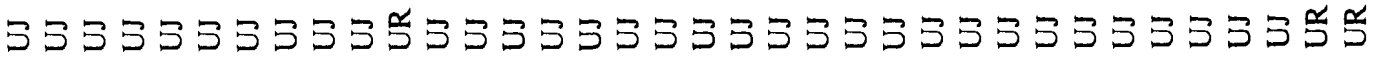

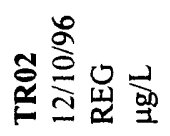

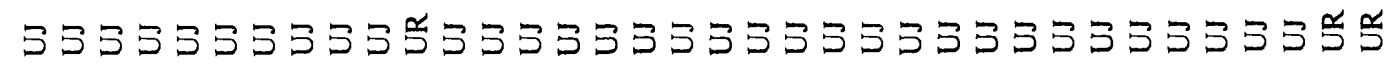

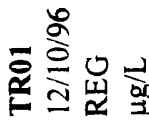

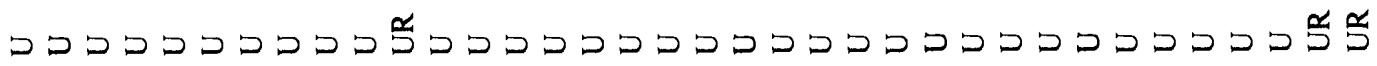

ถ

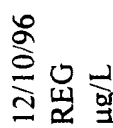

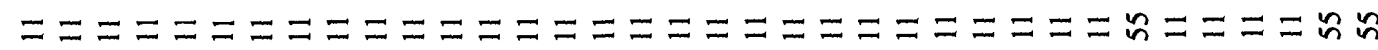

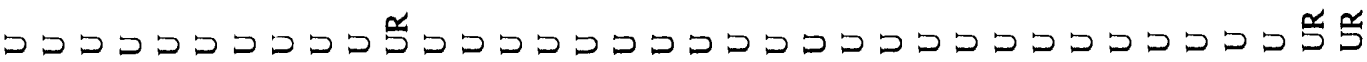

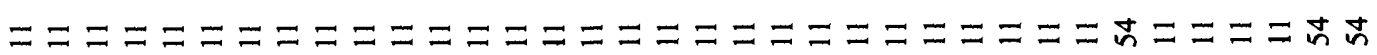

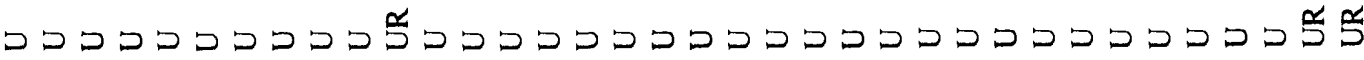

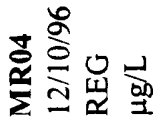

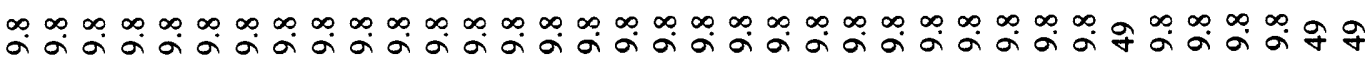

ڤ్

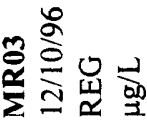

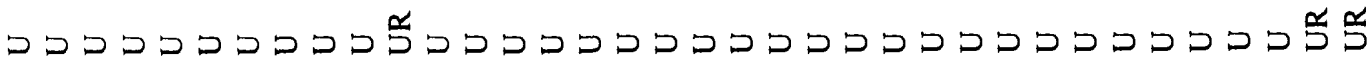

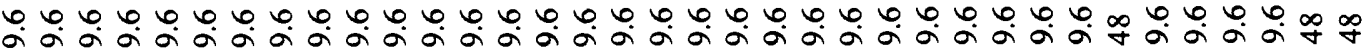

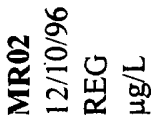

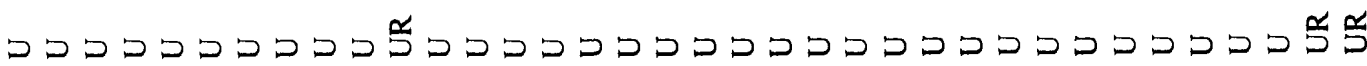

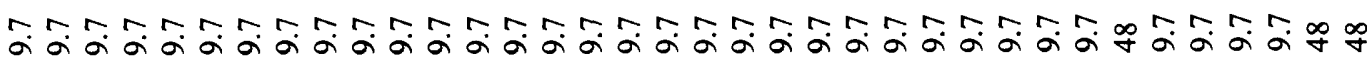

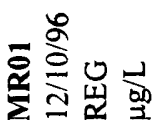

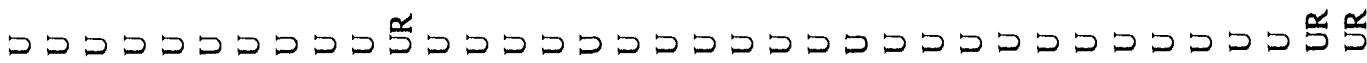

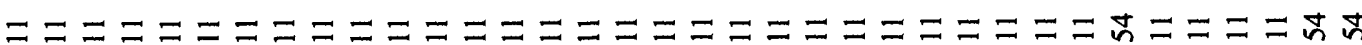
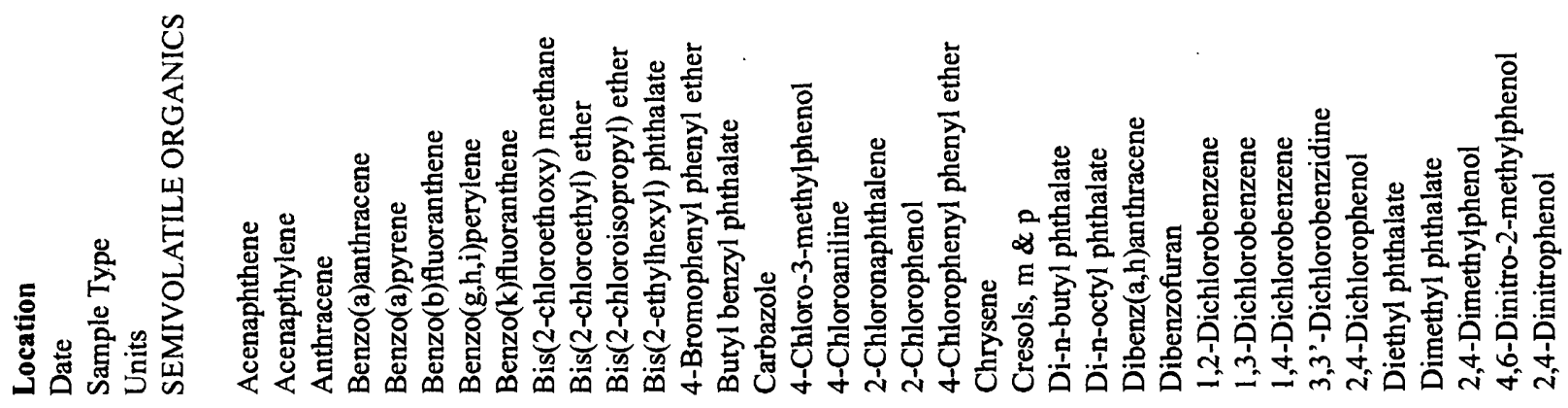


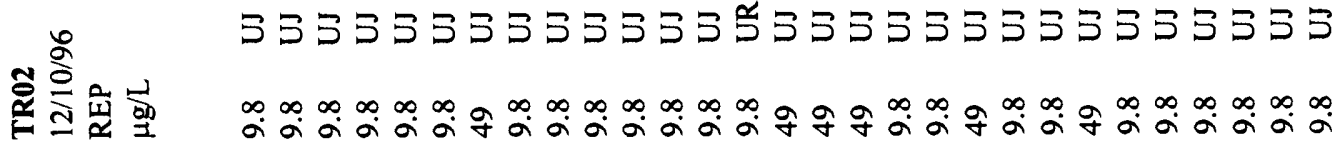

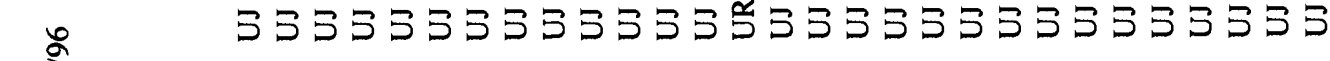

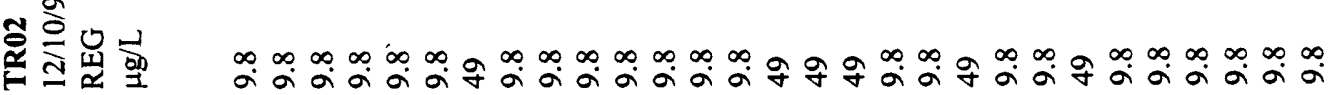

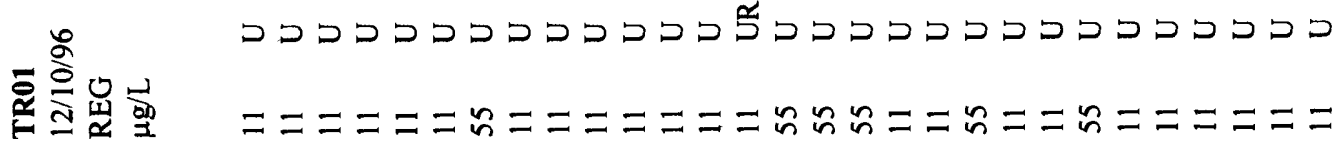

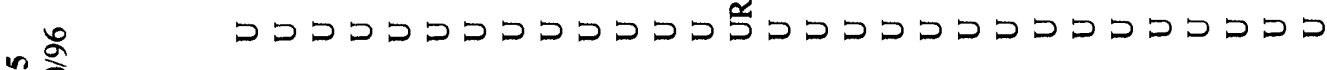

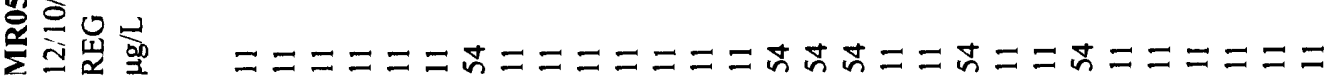

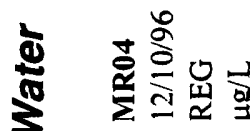

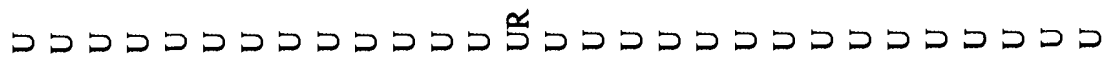

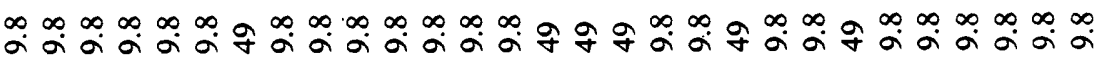

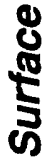

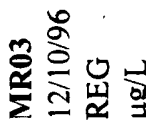

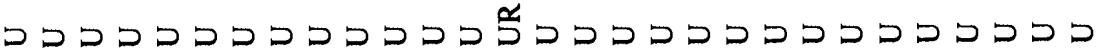

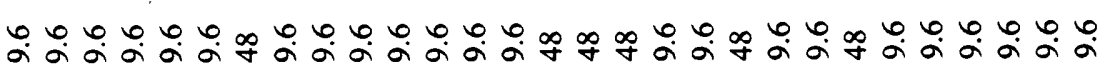

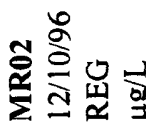

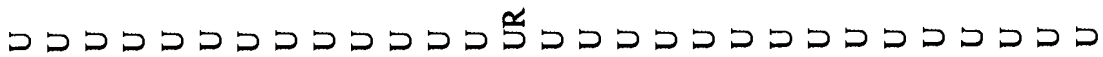

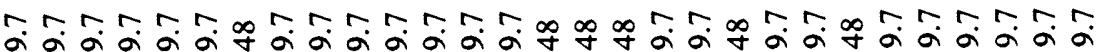

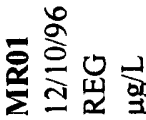

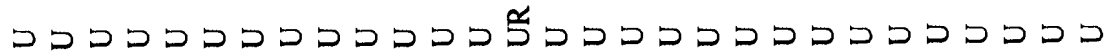

ニニニニニニ芯ニニニニニニニ芯芯芯ニニ芯ニニ芯ニニニニニニ

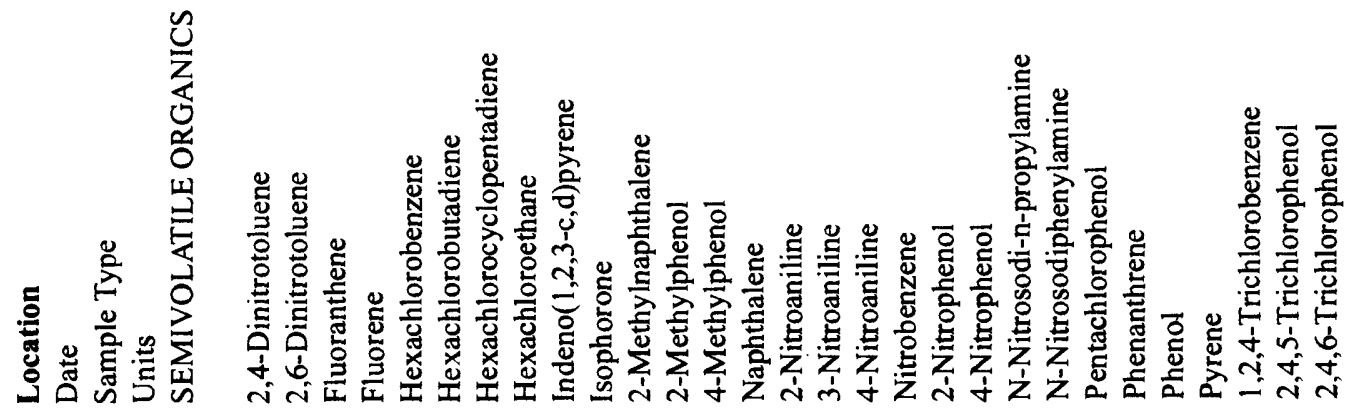




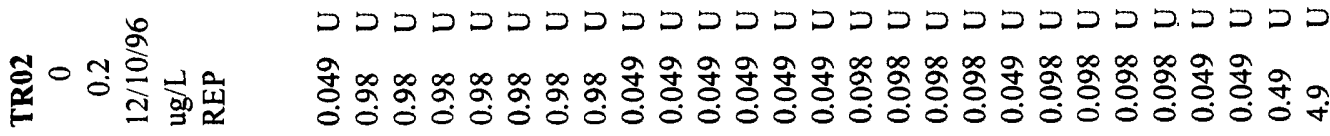

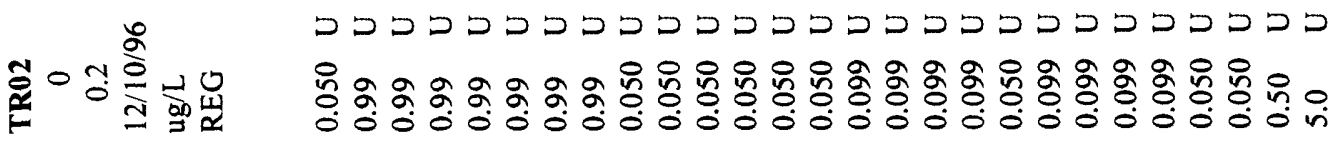

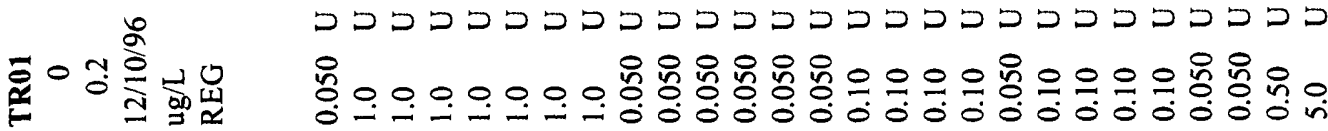

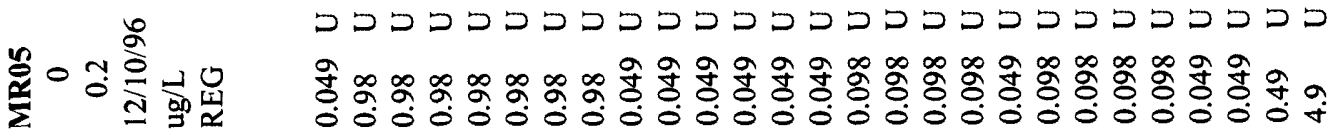

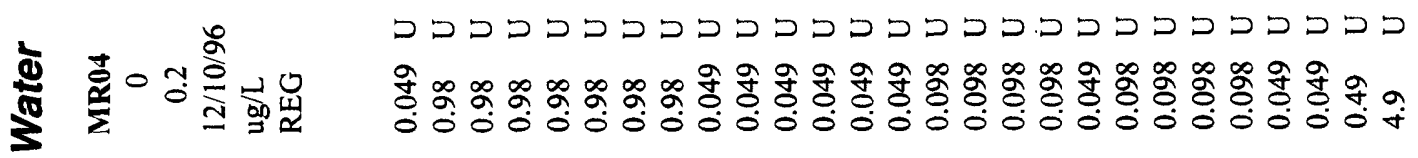

密

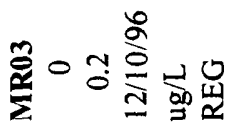

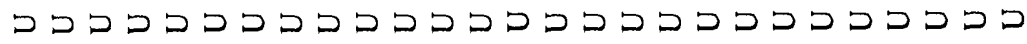

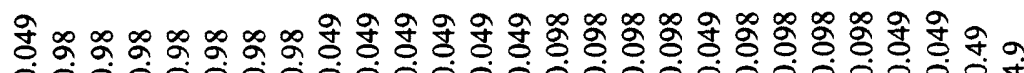

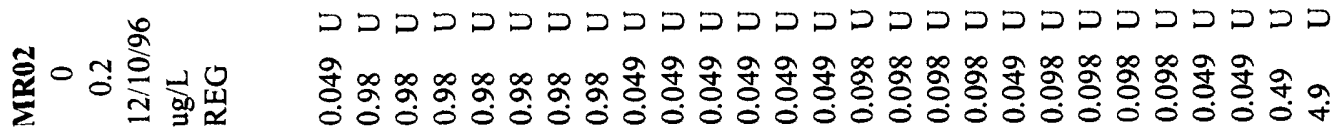

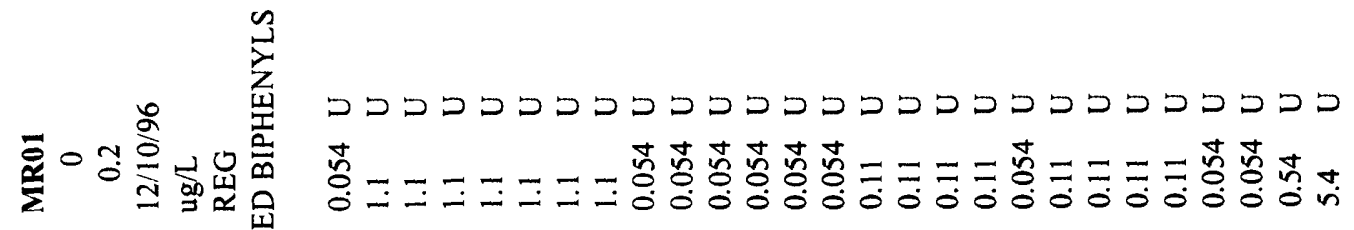




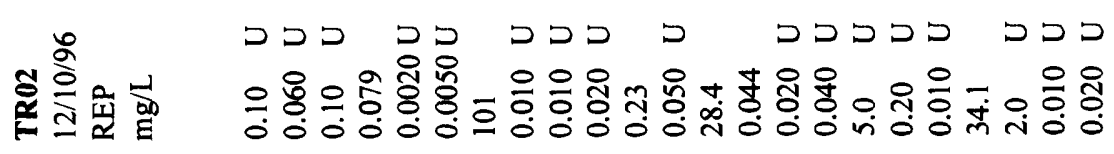

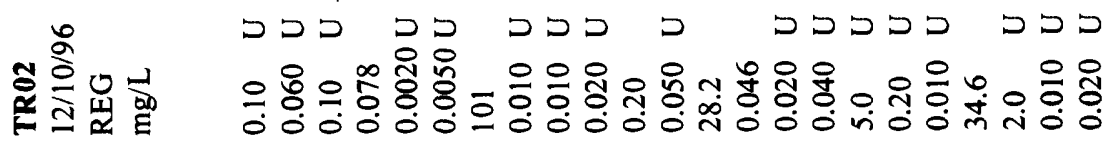

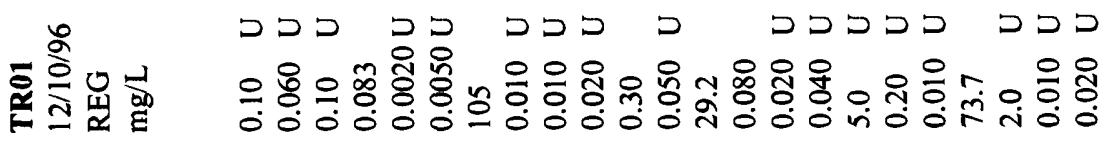

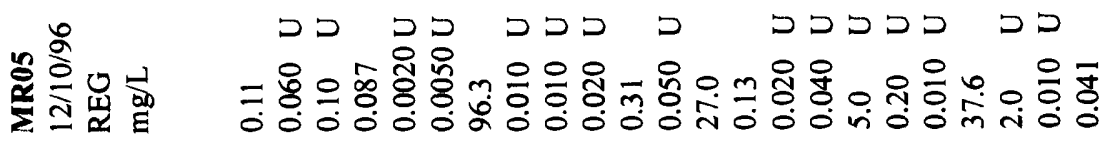

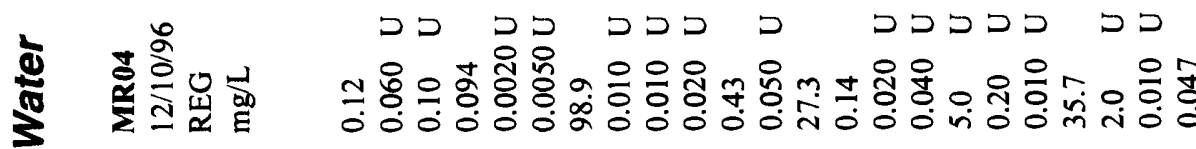

ڤั

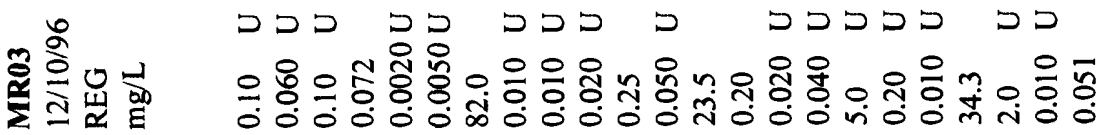

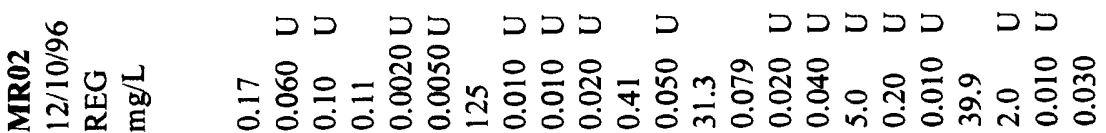

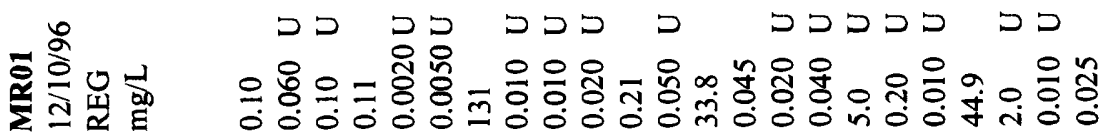

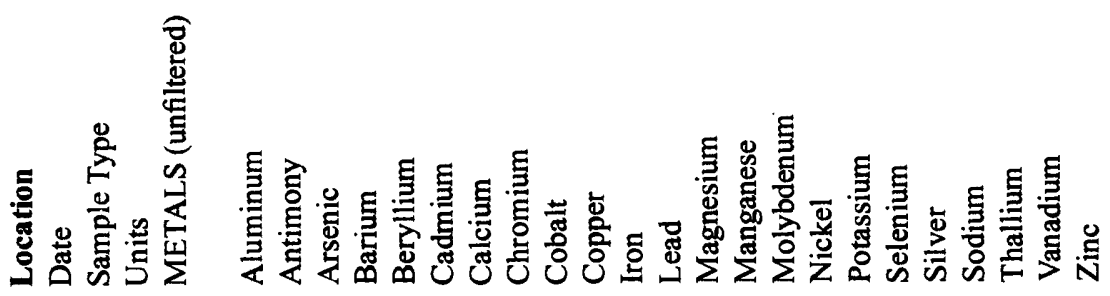




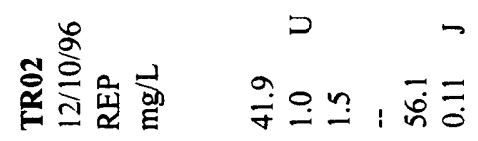

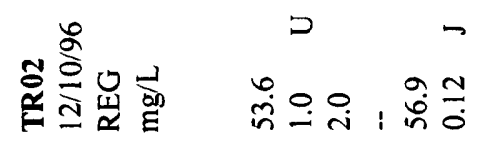

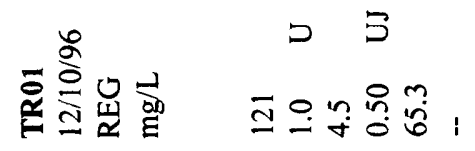

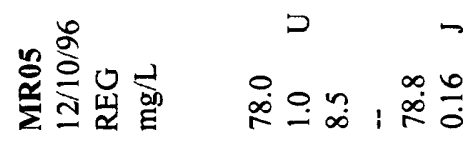

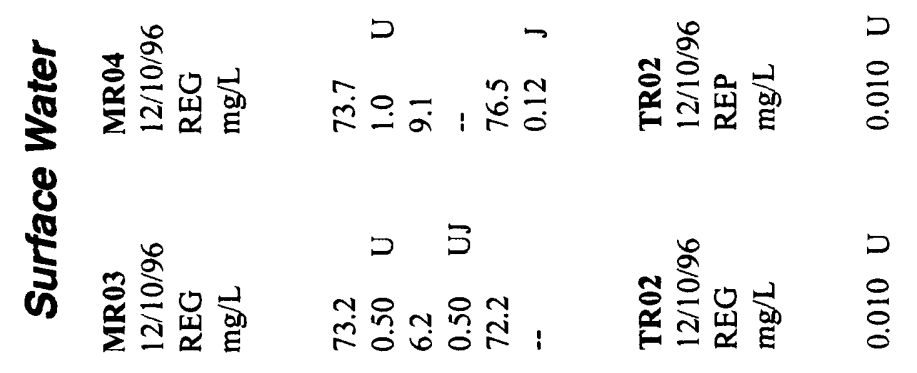

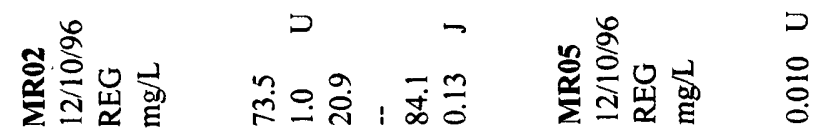

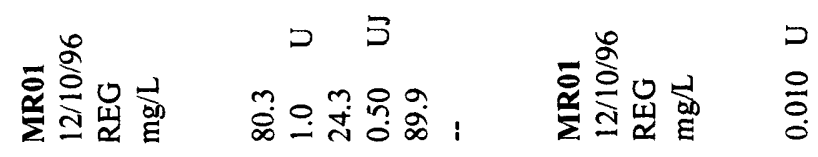

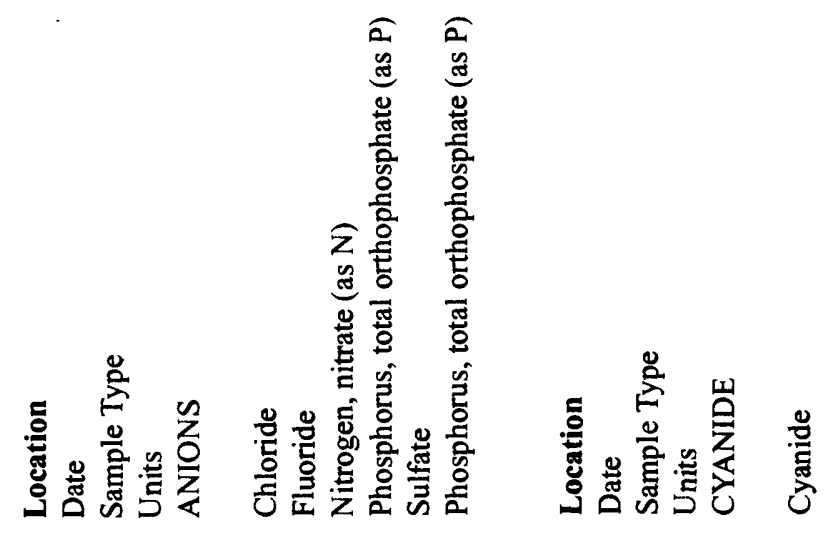




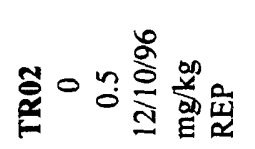

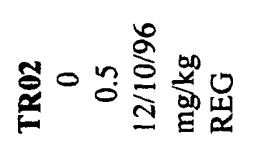

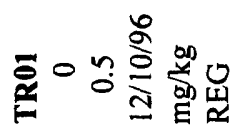

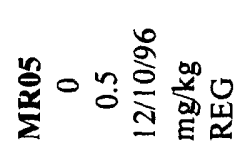

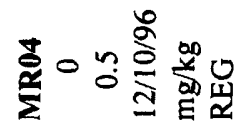

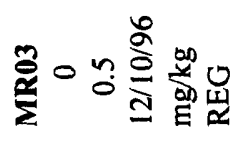

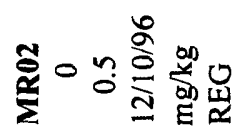

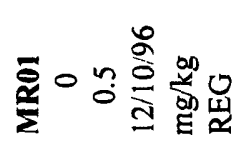

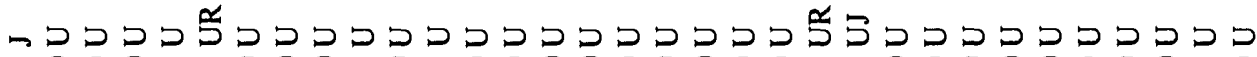

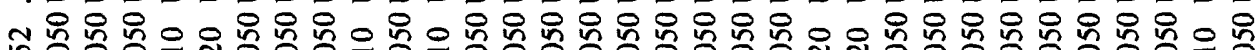

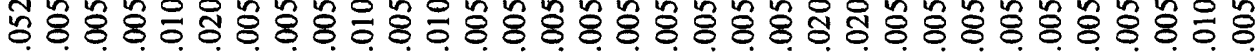

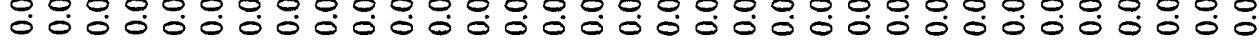

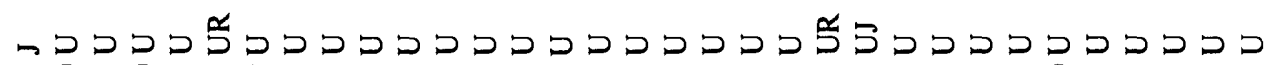

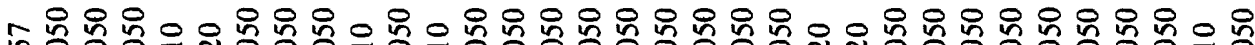
彳ิ

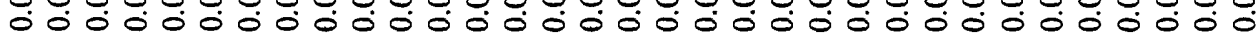

ح

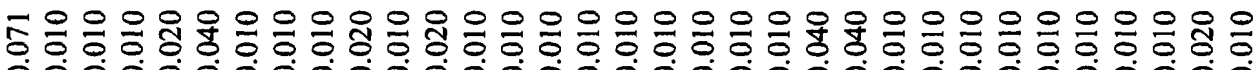

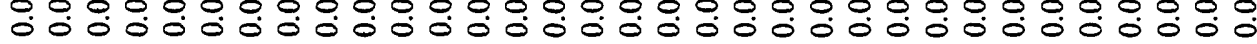

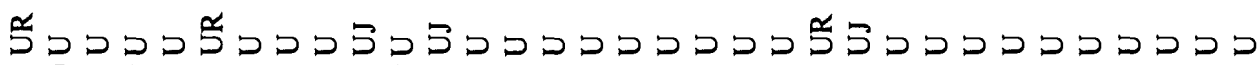

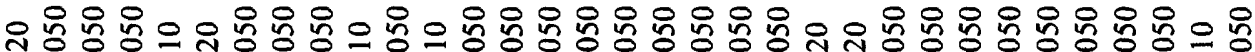

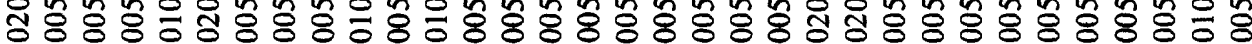

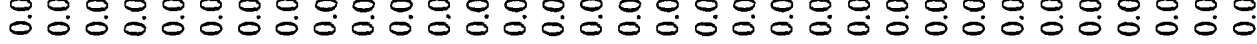

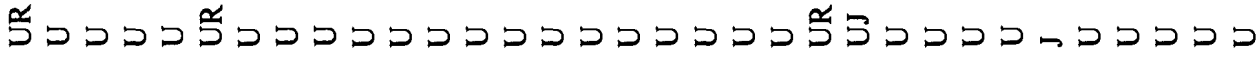

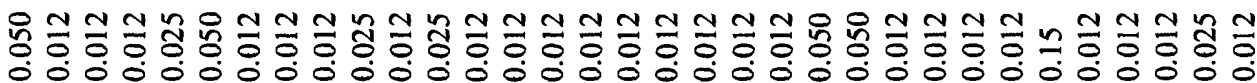

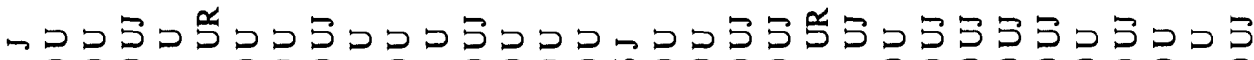

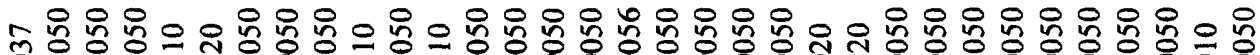

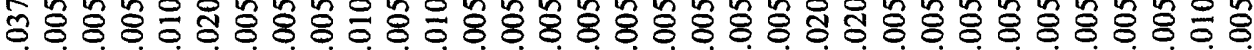

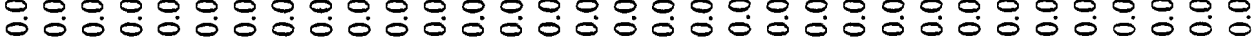

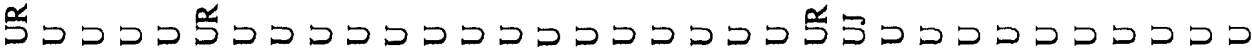

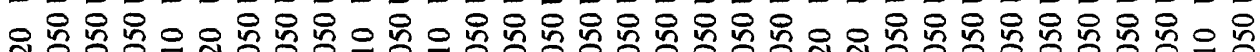

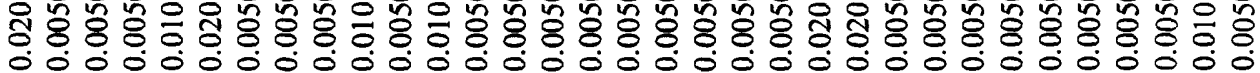

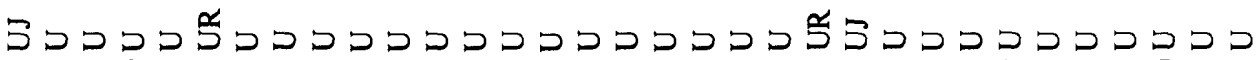

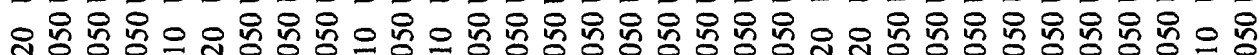

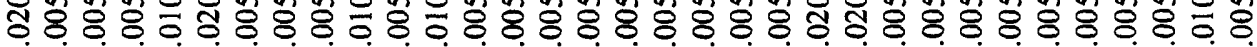

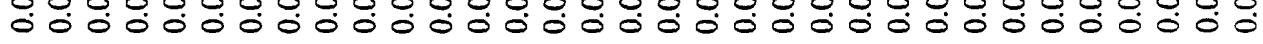
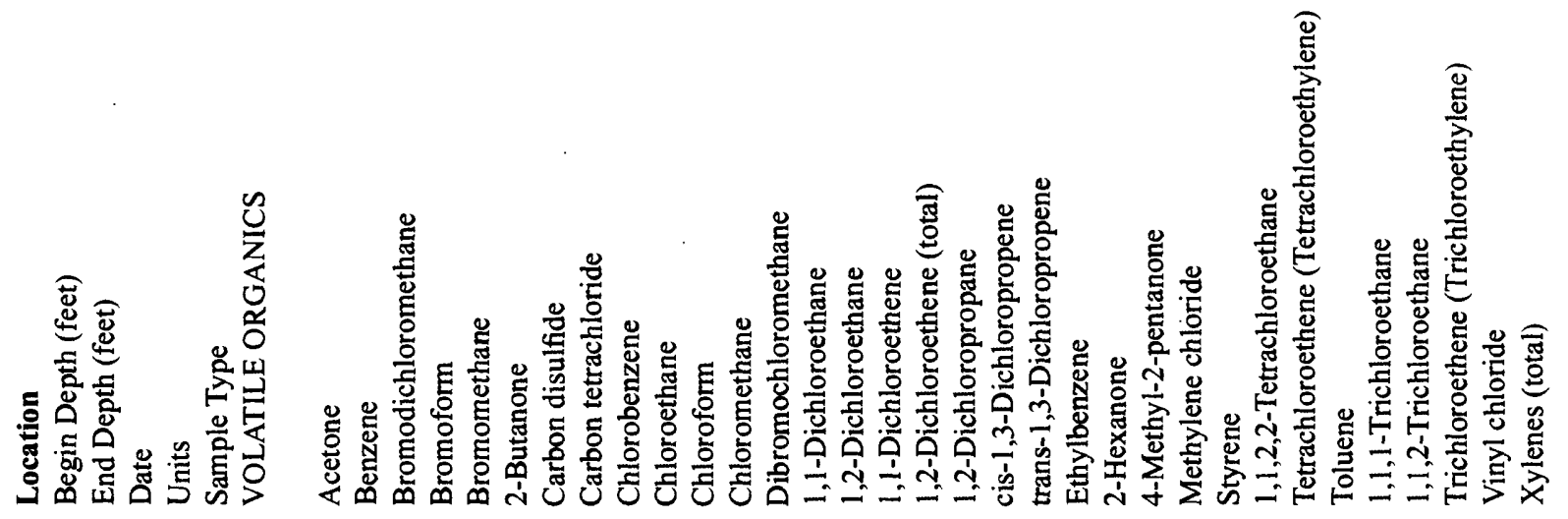


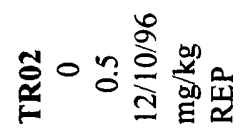

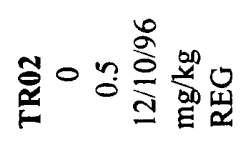

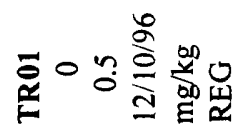

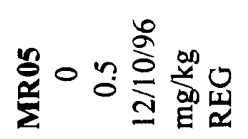

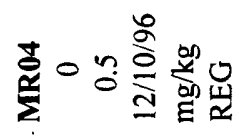

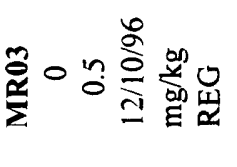

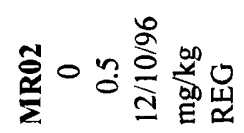

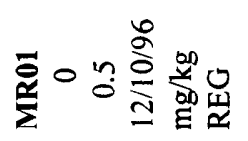

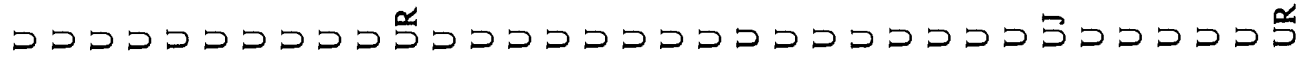
بِ

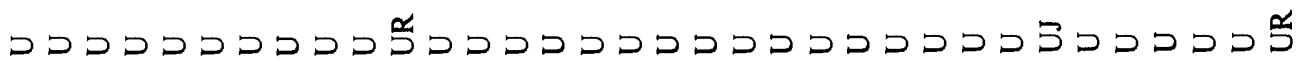
ف요

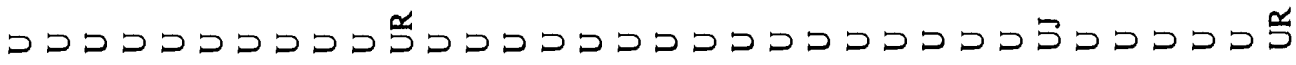

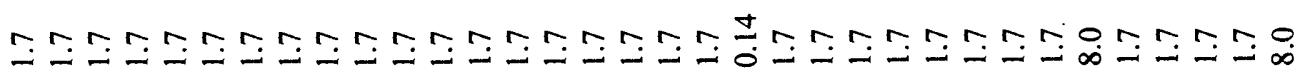

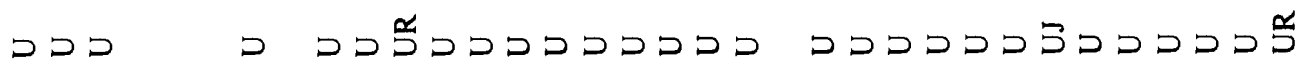

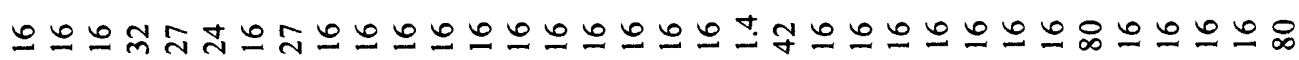

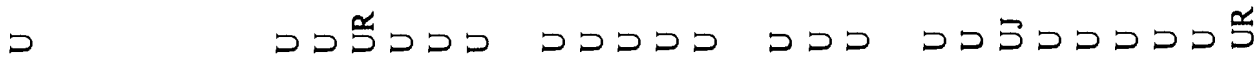

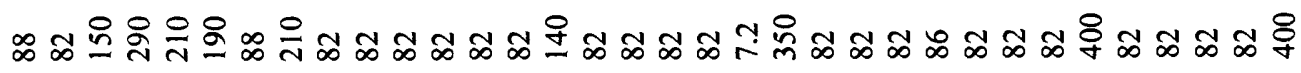

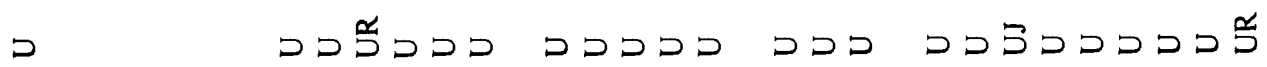

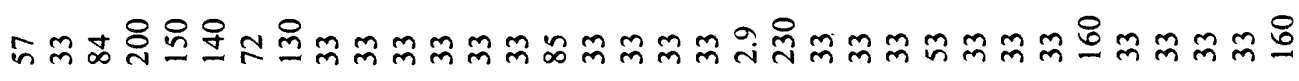

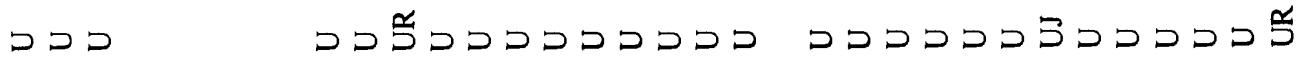

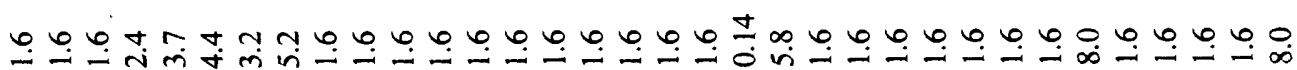

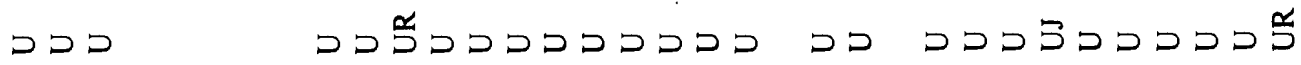

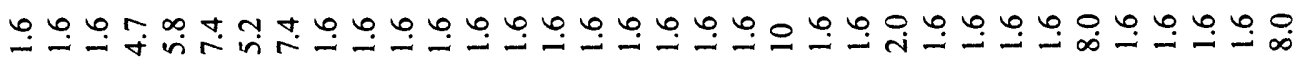
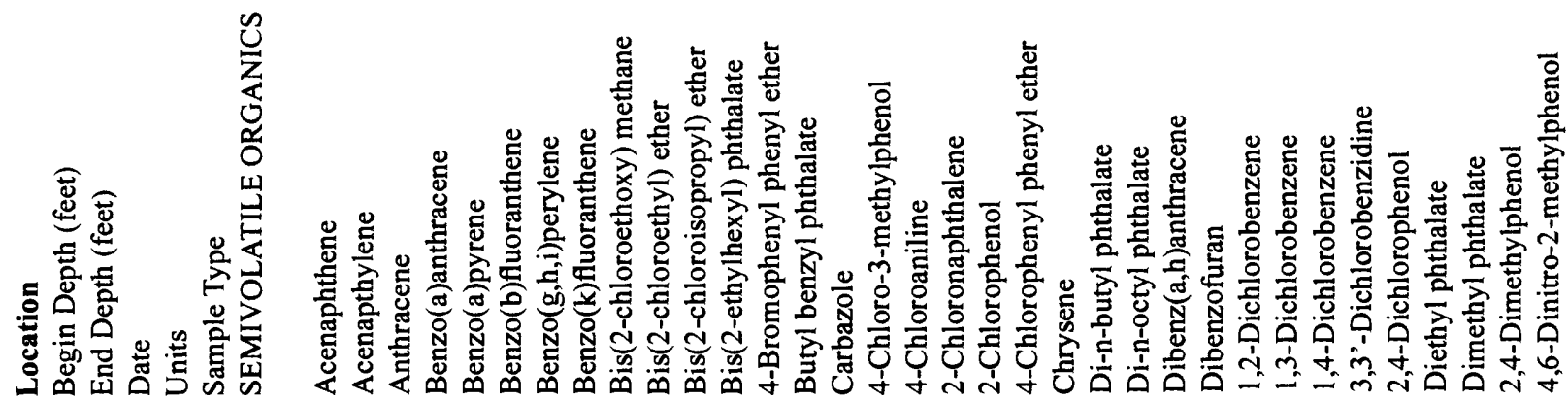


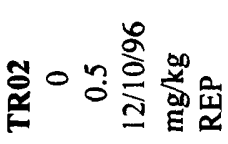

잉

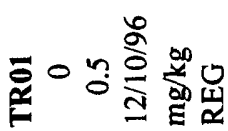

纟o

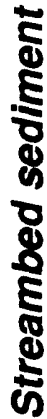

¿

๕on

올

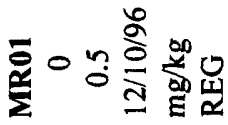

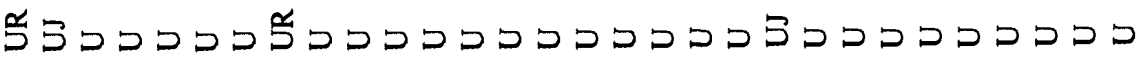
○

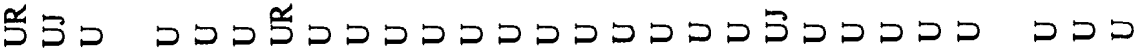
○

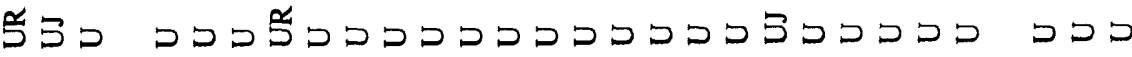

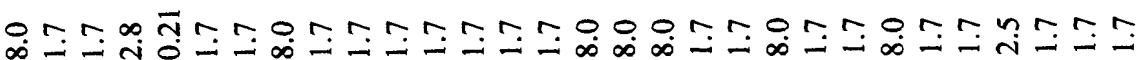

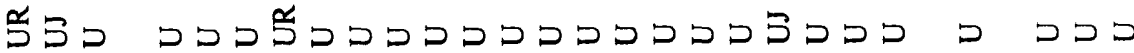

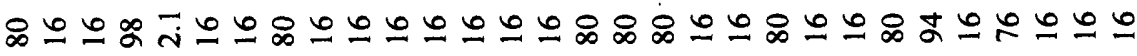

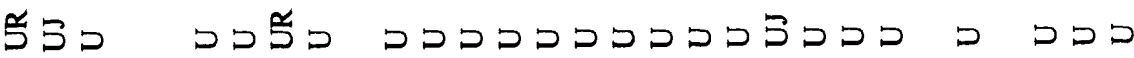
중 쥬

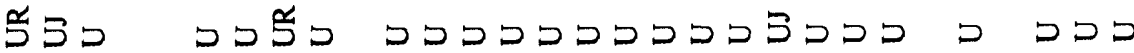
을 m

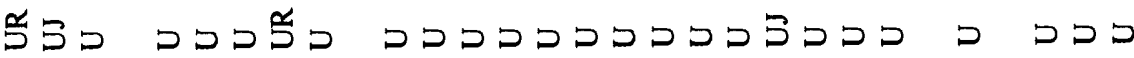
o

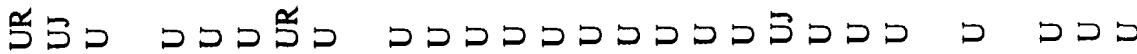
oํ
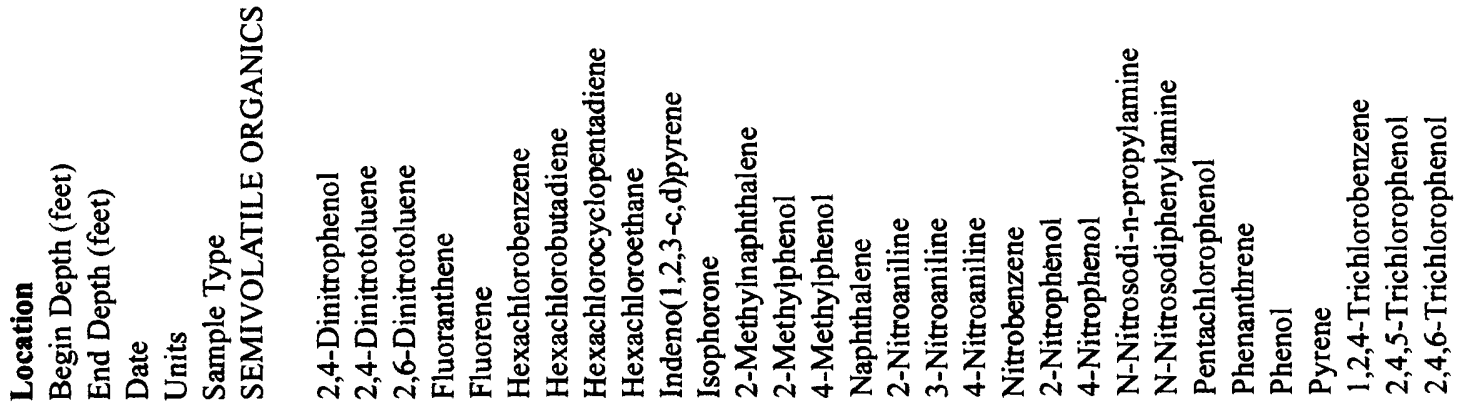


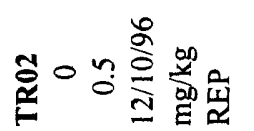

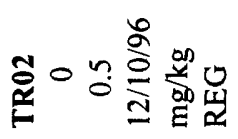

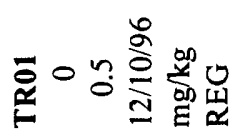

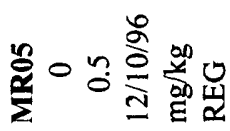

ฐิ

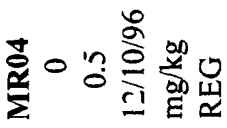

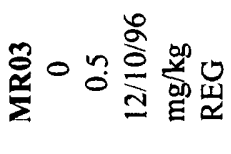

突ㅇำ

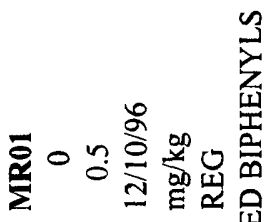

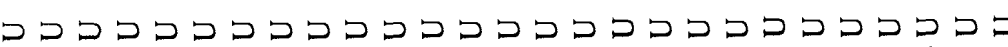

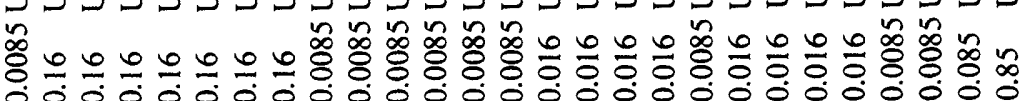

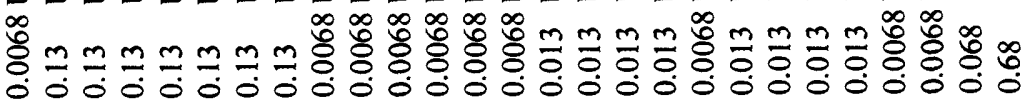

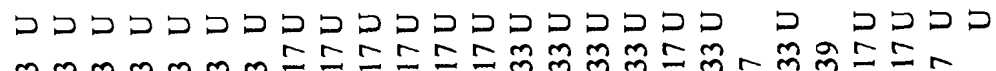

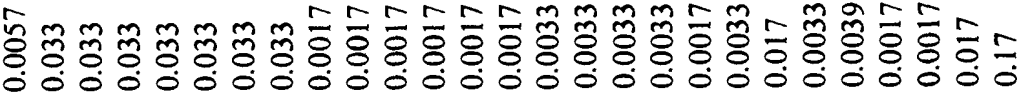

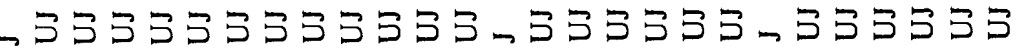

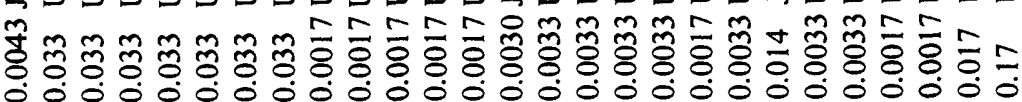

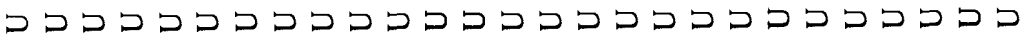

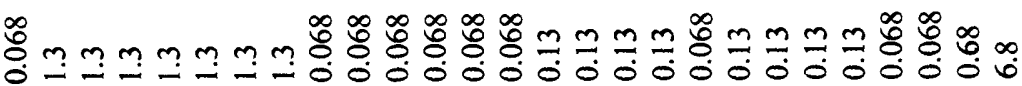

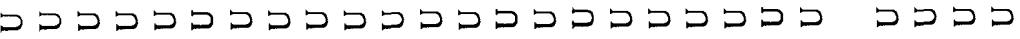

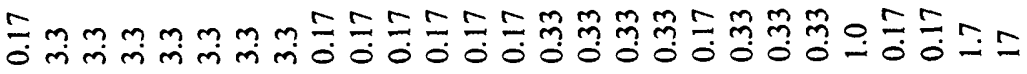

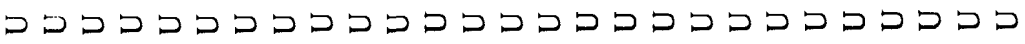

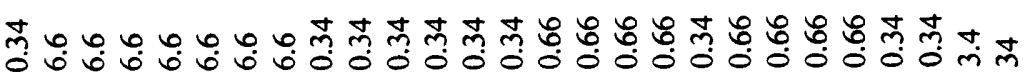

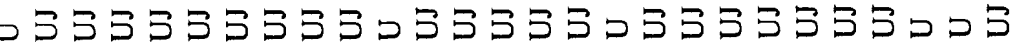

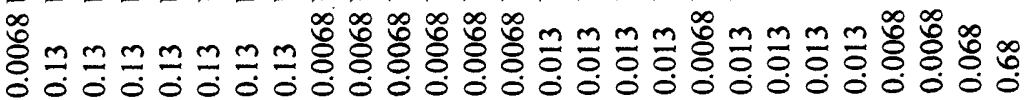

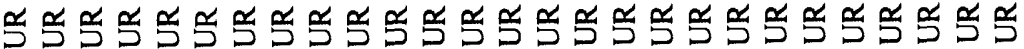

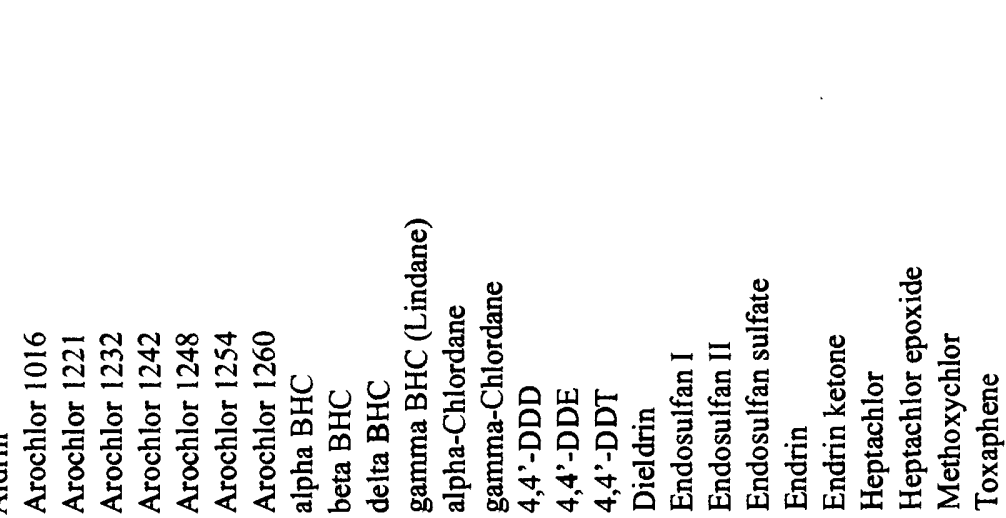




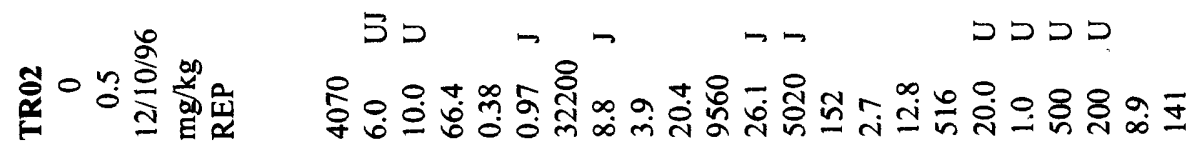

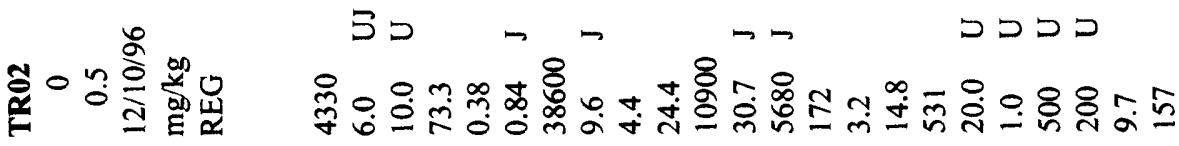

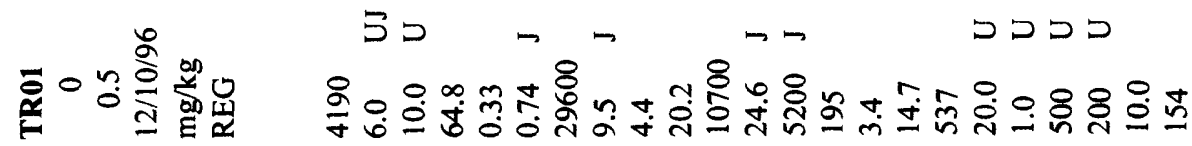

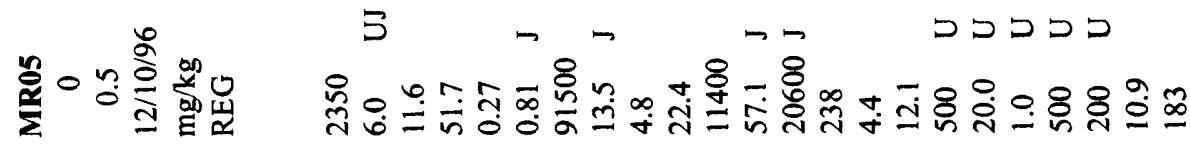

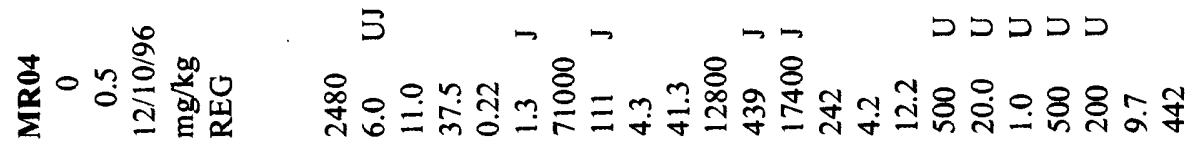

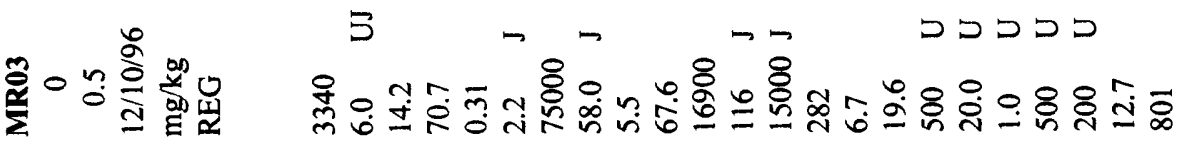

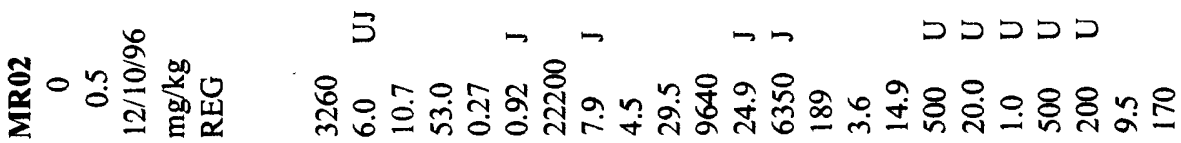

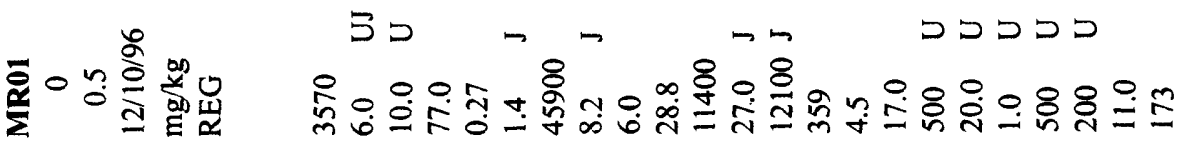

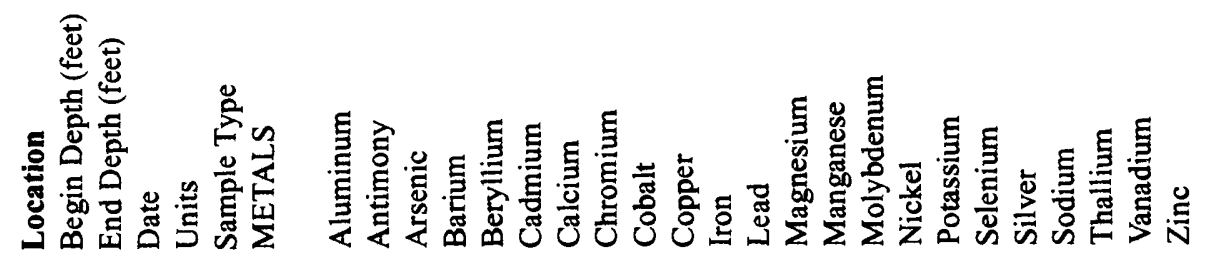



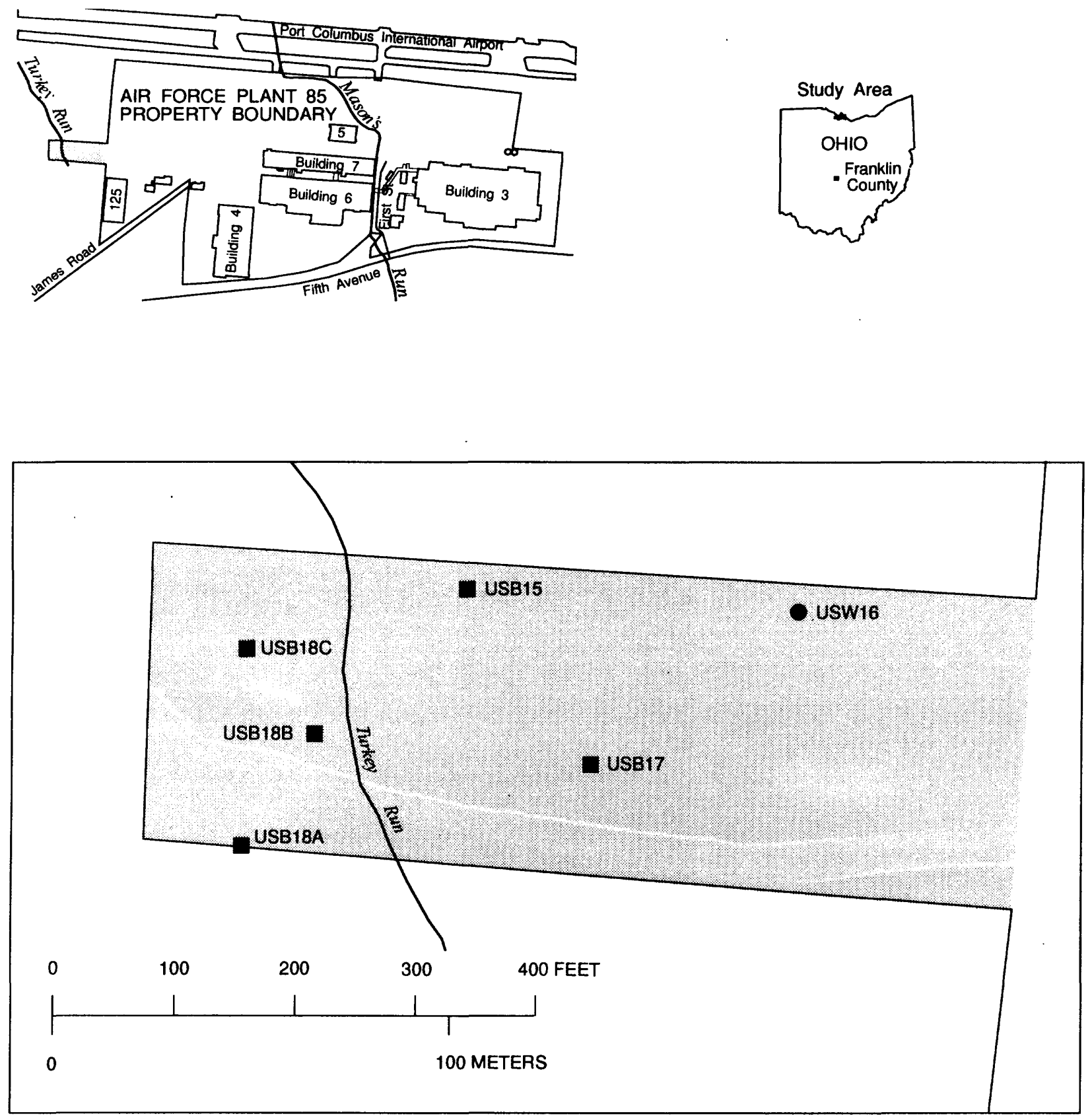

\section{EXPLANATION}

SAMPLING AREA 1

MONITORING WELL LOCATION AND IDENTIFIER

SOIL-SAMPLE BOREHOLE LOCATION AND IDENTIFIER

Figure 3. Soil boring/sampling locations at the magnesium-chip burning and rubble-disposal area, Air Force Plant 85, Columbus, Ohio. 

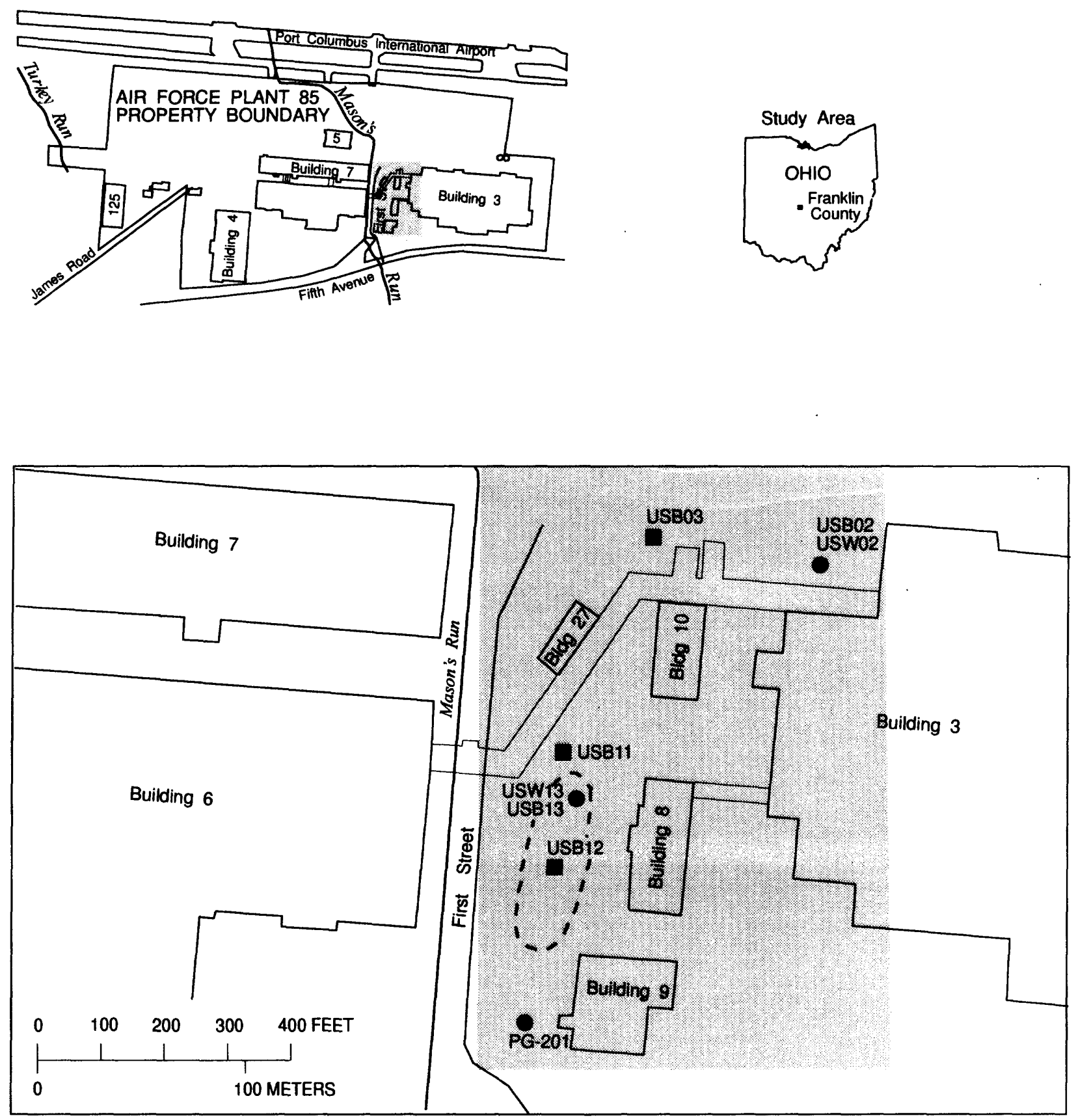

\section{EXPLANATION}

SAMPLING AREA 2

- - - EXTENT OF COAL PILE

- MONITORING WELL LOCATION AND IDENTIFIER

- SOIL-SAMPLE BOREHOLE LOCATION AND IDENTIFIER

Figure 4. Soil boring/sampling locations near the coal pile, Air Force Plant 85 , Columbus, Ohio. 

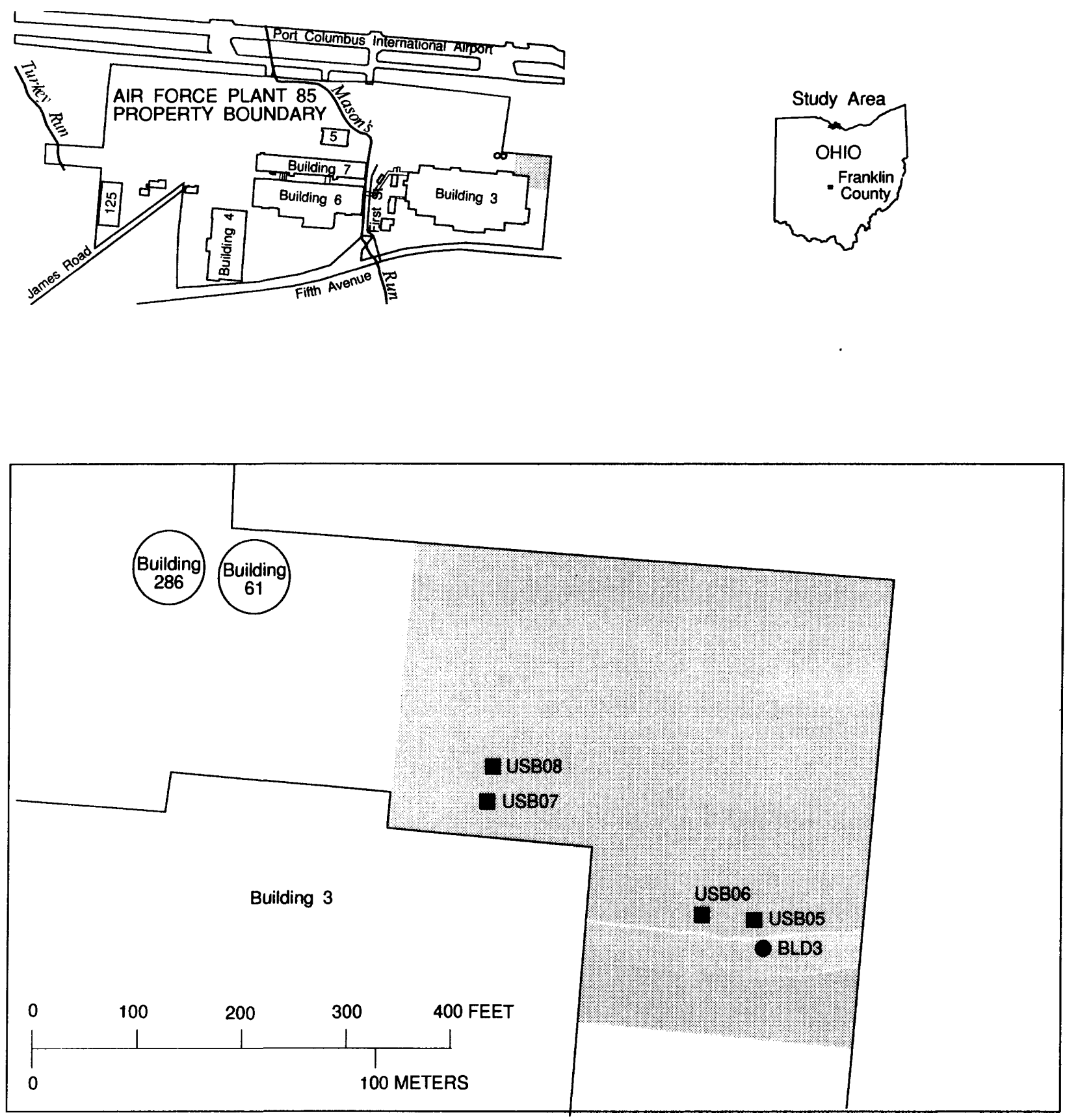

EXPLANATION

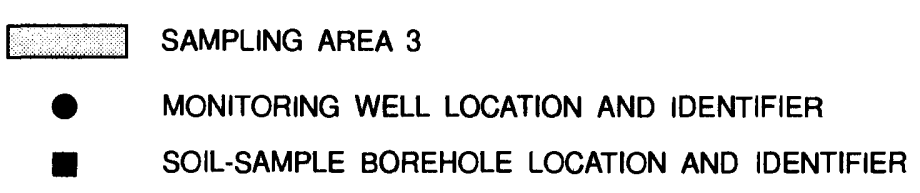

Figure 5. Soil boring/sampling locations north of Building 3 and at fuel tank area east of Building 3, Air Force Plant 85, Columbus, Ohio. 

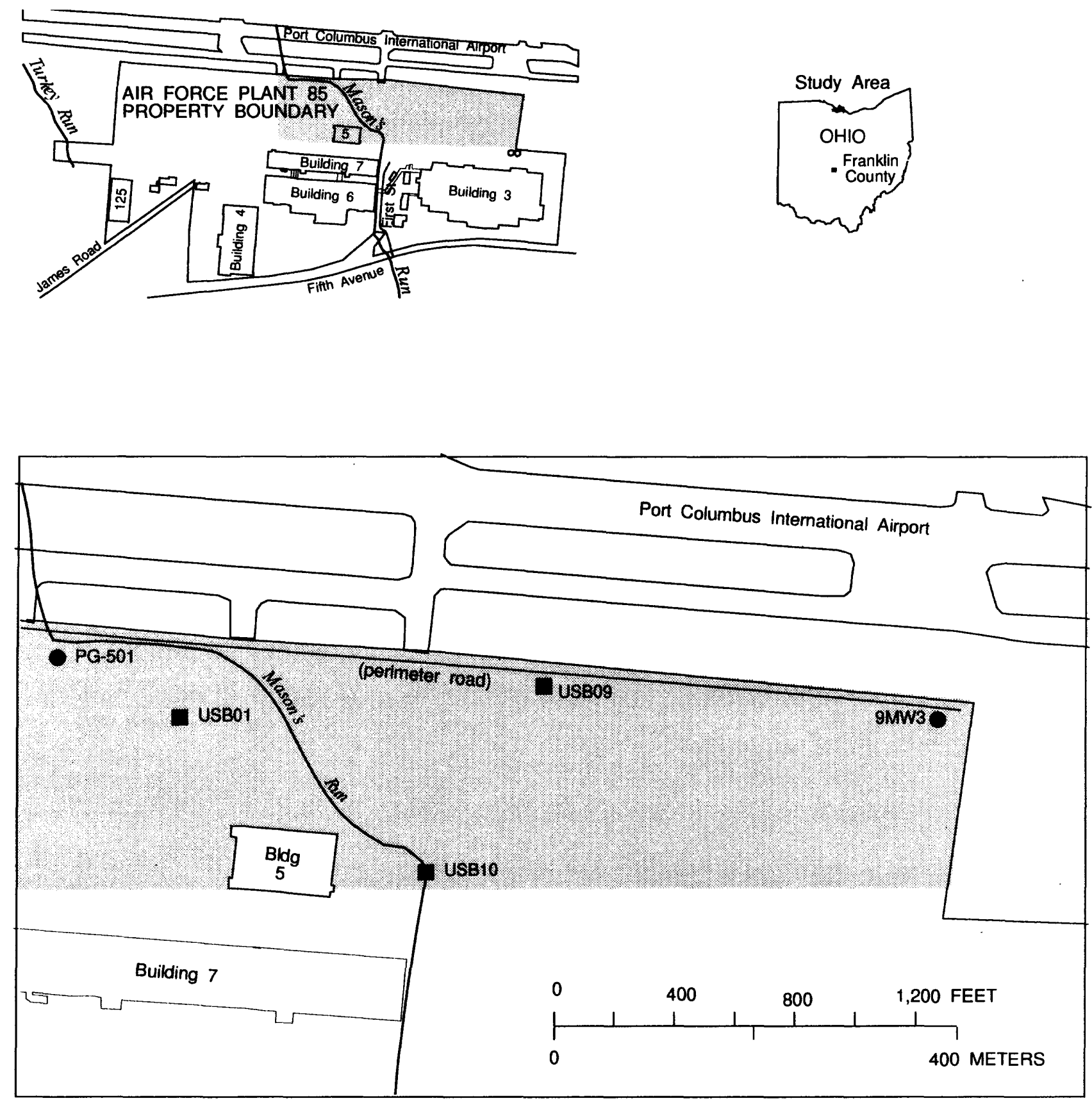

\section{EXPLANATION}

Figure 6. Soil boring/sampling locations on the north side of Air Force Plant 85 (north-central boundary, wash rack area, and near Mason's Run), Columbus, Ohio. 

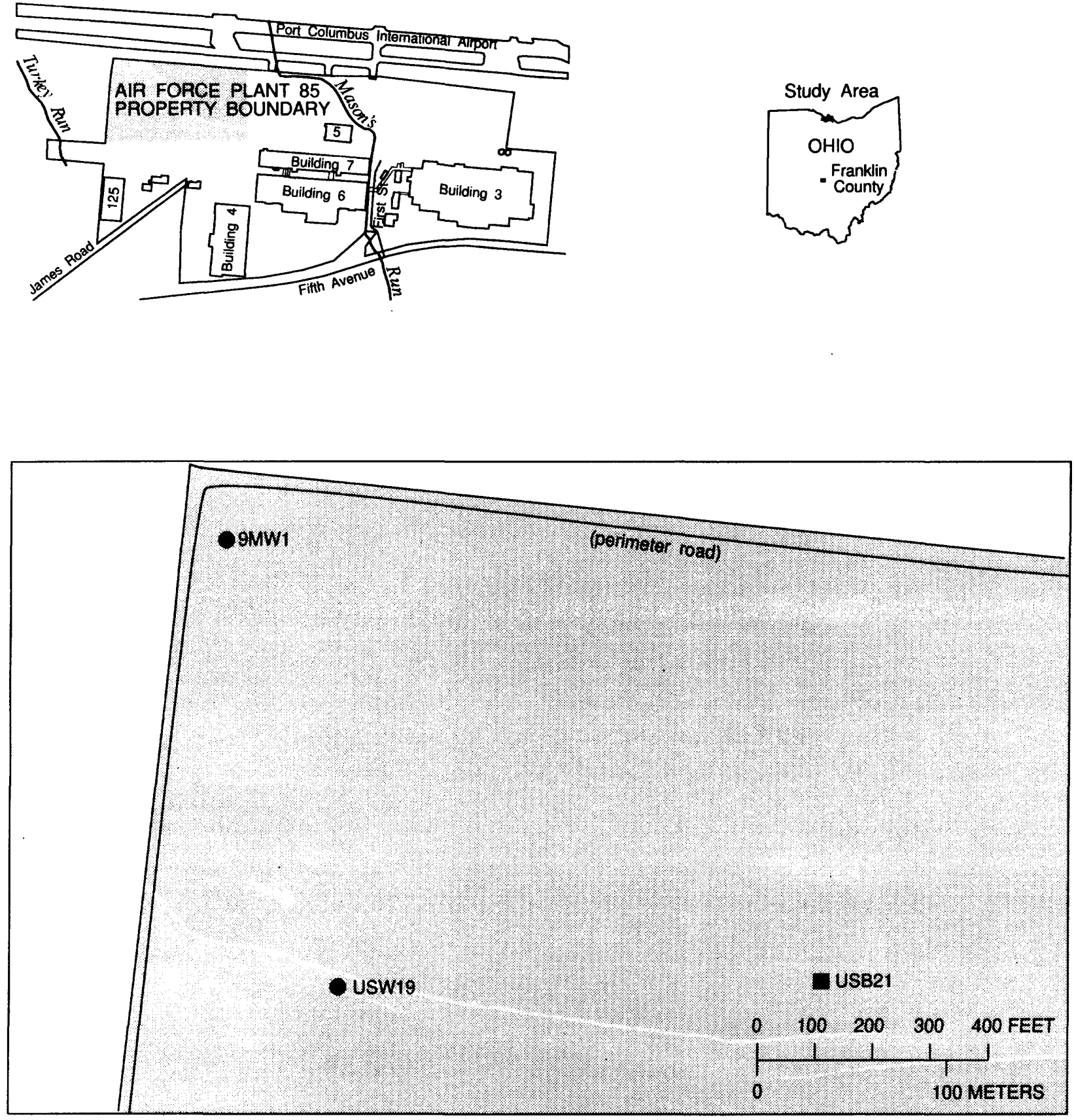

EXPLANATION

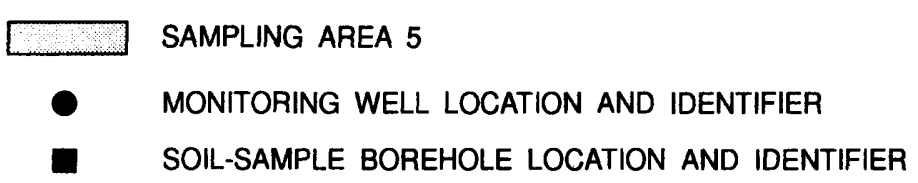

Figure 7. Soil boring/sampling locations at leach-field area, stationary radar site, and northwest perimeter road, Air Force Plant 85 , Columbus, Ohio. 

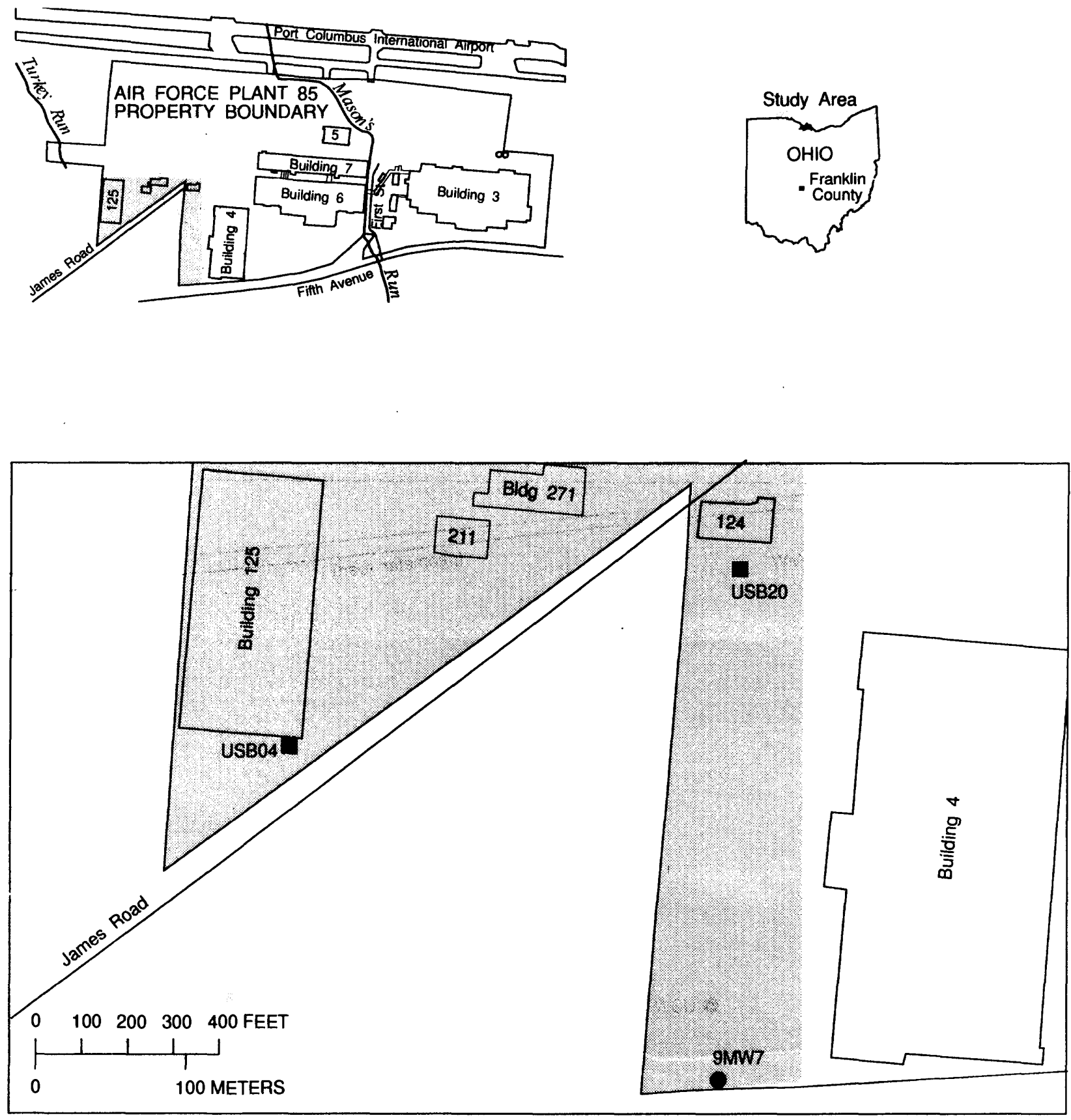

\section{EXPLANATION}

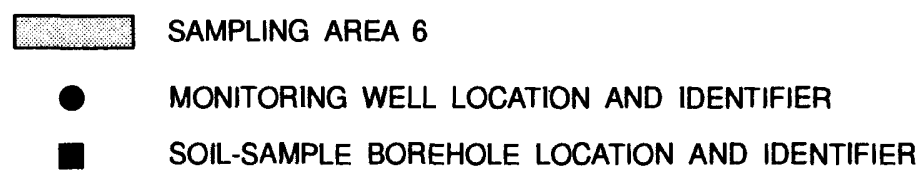

Figure 8. Soil boring/sampling locations on the southwestern part of Air Force Plant 85 (south of Bulldings 124 and 125 and in southwest Building 4 parking lot), Columbus, Ohio. 

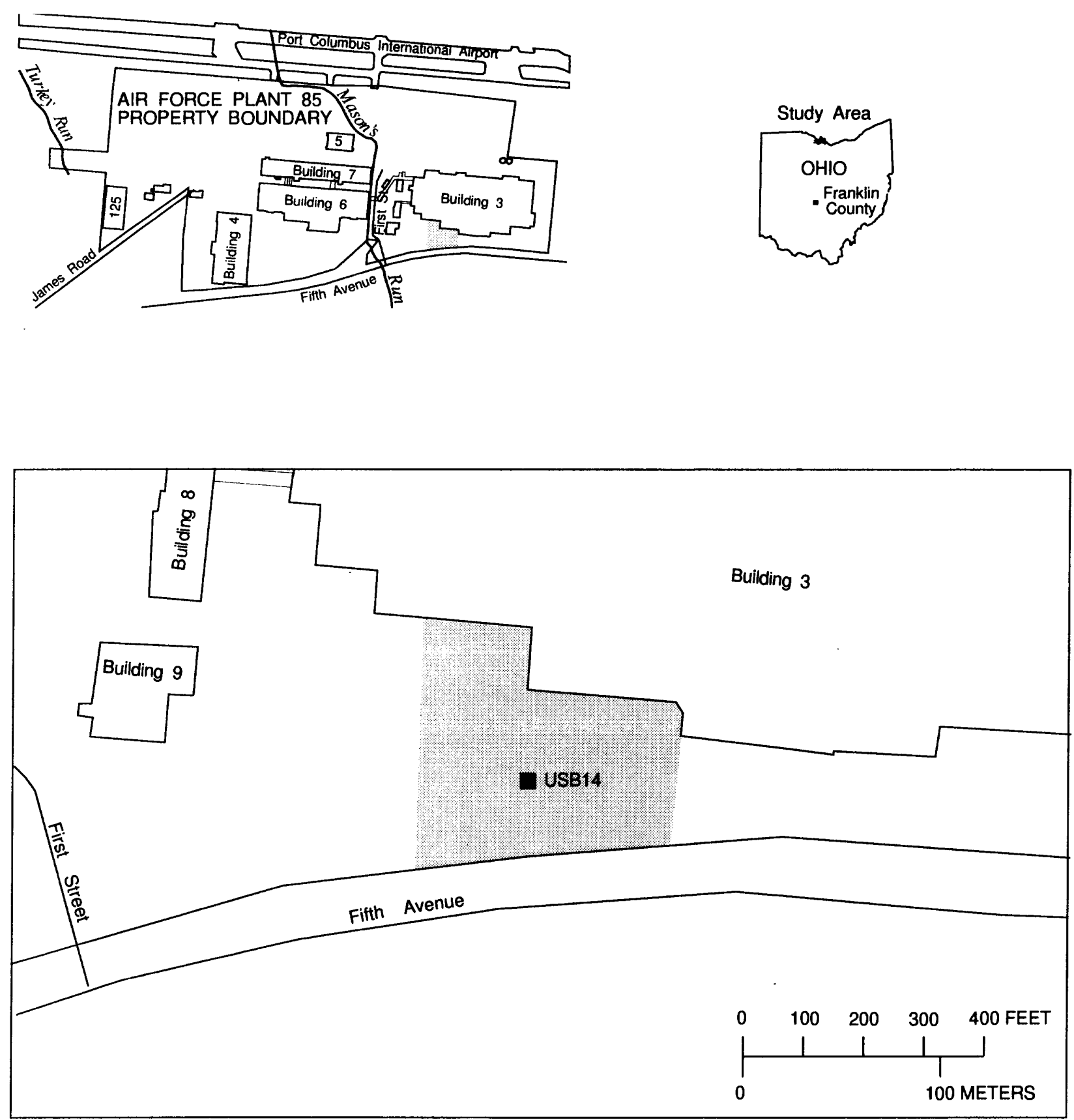

EXPLANATION

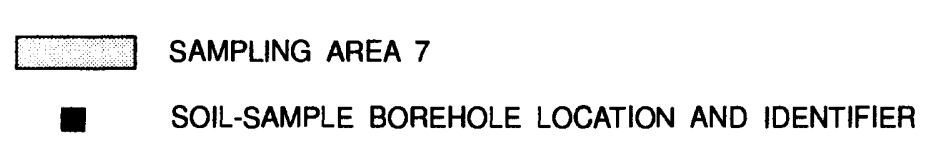

Figure 9. Soil boring/sampling locations southwest part of Building 3 (in parking lot), Air Force Plant 85, Columbus, Ohio. 

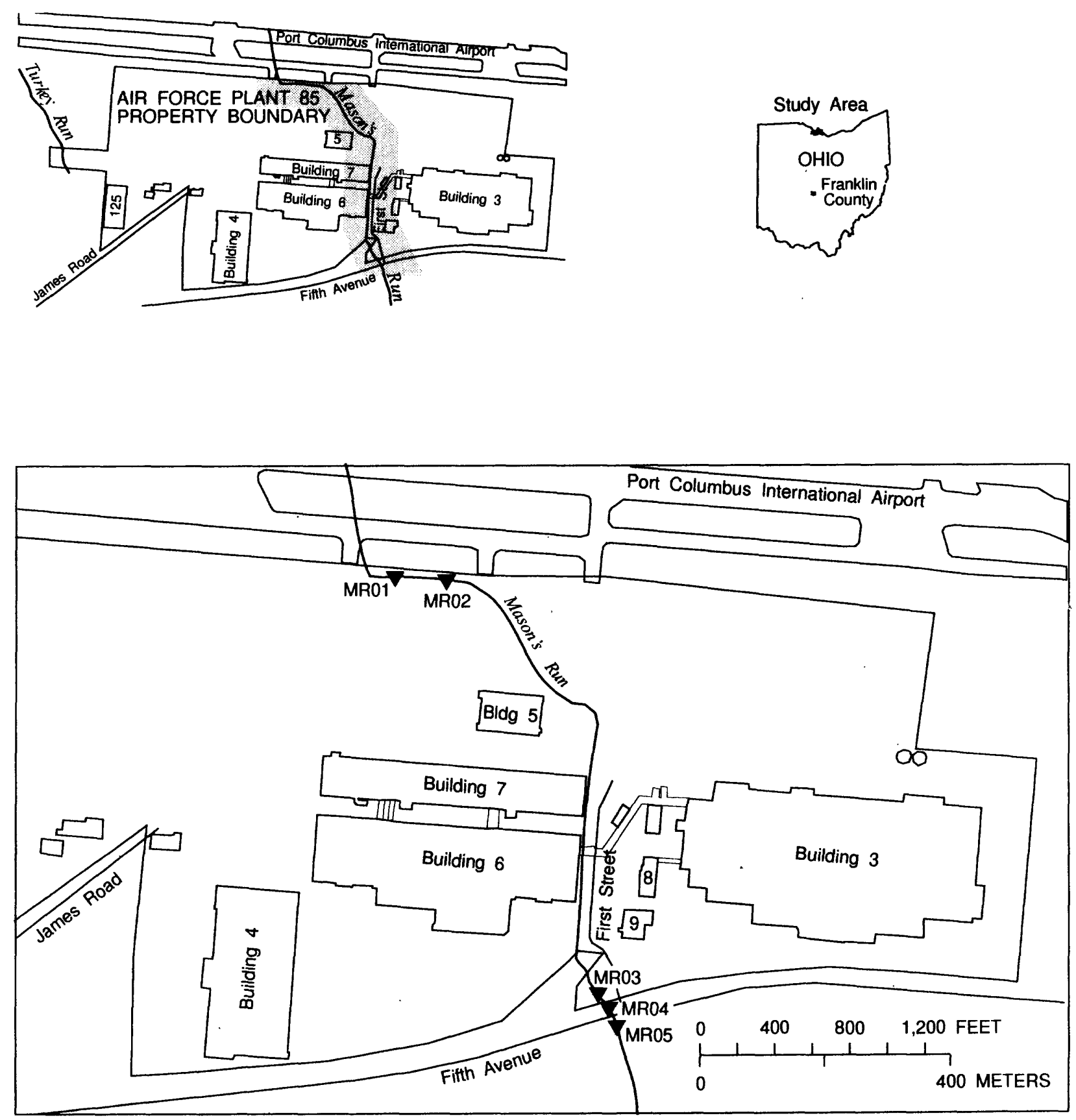

\section{EXPLANATION}

SURFACE-WATER AND STREAMBED-SAMPLING SITE WITH IDENTIFIER

Figure 10. Surface-water and streambed-sediment sampling locations on Mason's Run, Air Force Plant 85 , Columbus, Ohio. 

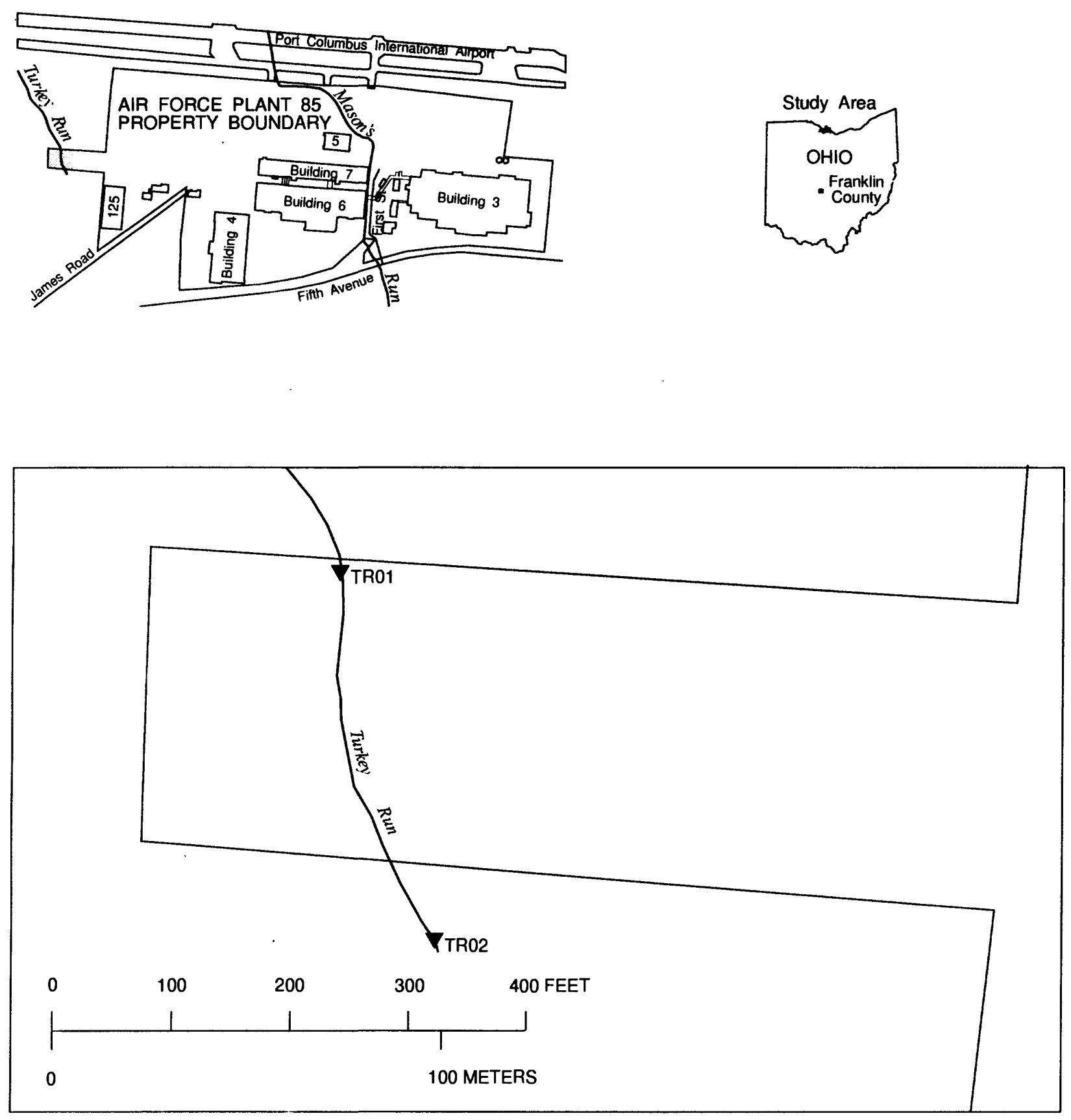

\section{EXPLANATION}

$\nabla$ SURFACE-WATER AND STREAMBED-SEDIMENT

SAMPLING SITE

Figure 11. Surface-water and streambed-sediment sampling locations on Turkey Run, Air Force Plant 85, Columbus, Ohio. 


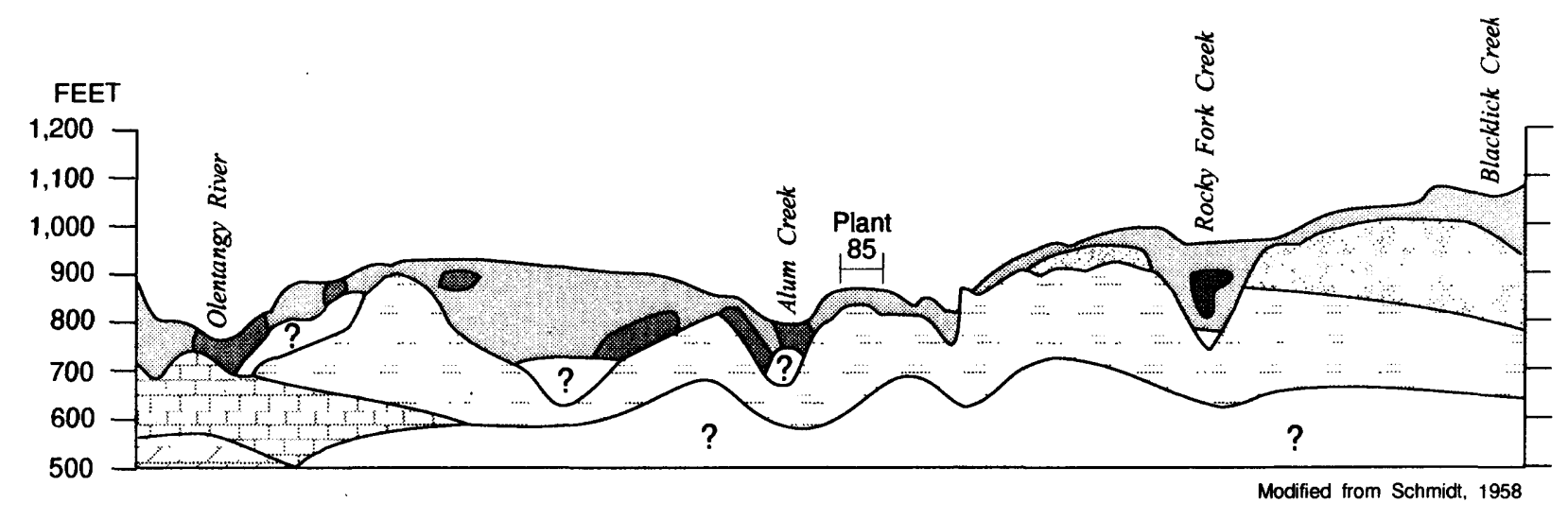

\section{EXPLANATION}
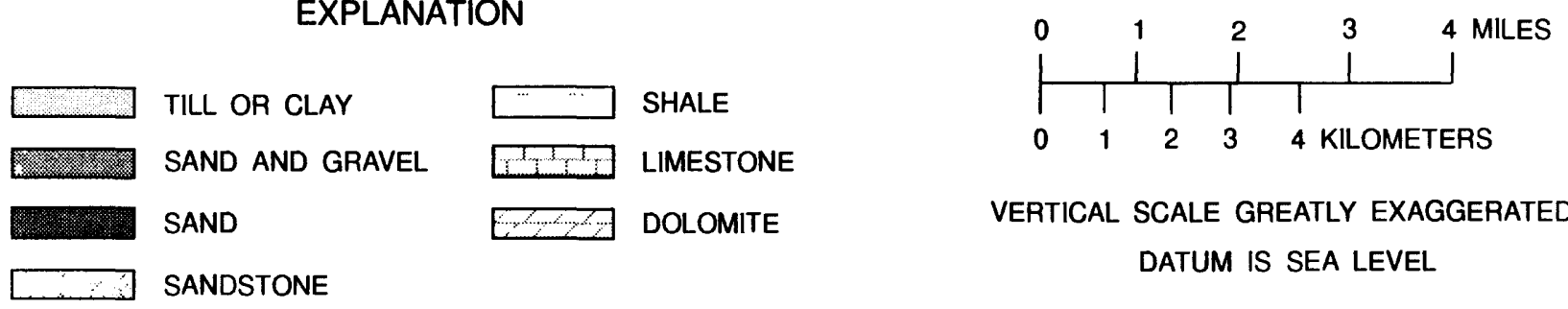

VERTICAL SCALE GREATLY EXAGGERATED DATUM IS SEA LEVEL

Figure 12. Generalized cross section showing geology of Air Force Plant 85, Columbus, Ohio. 

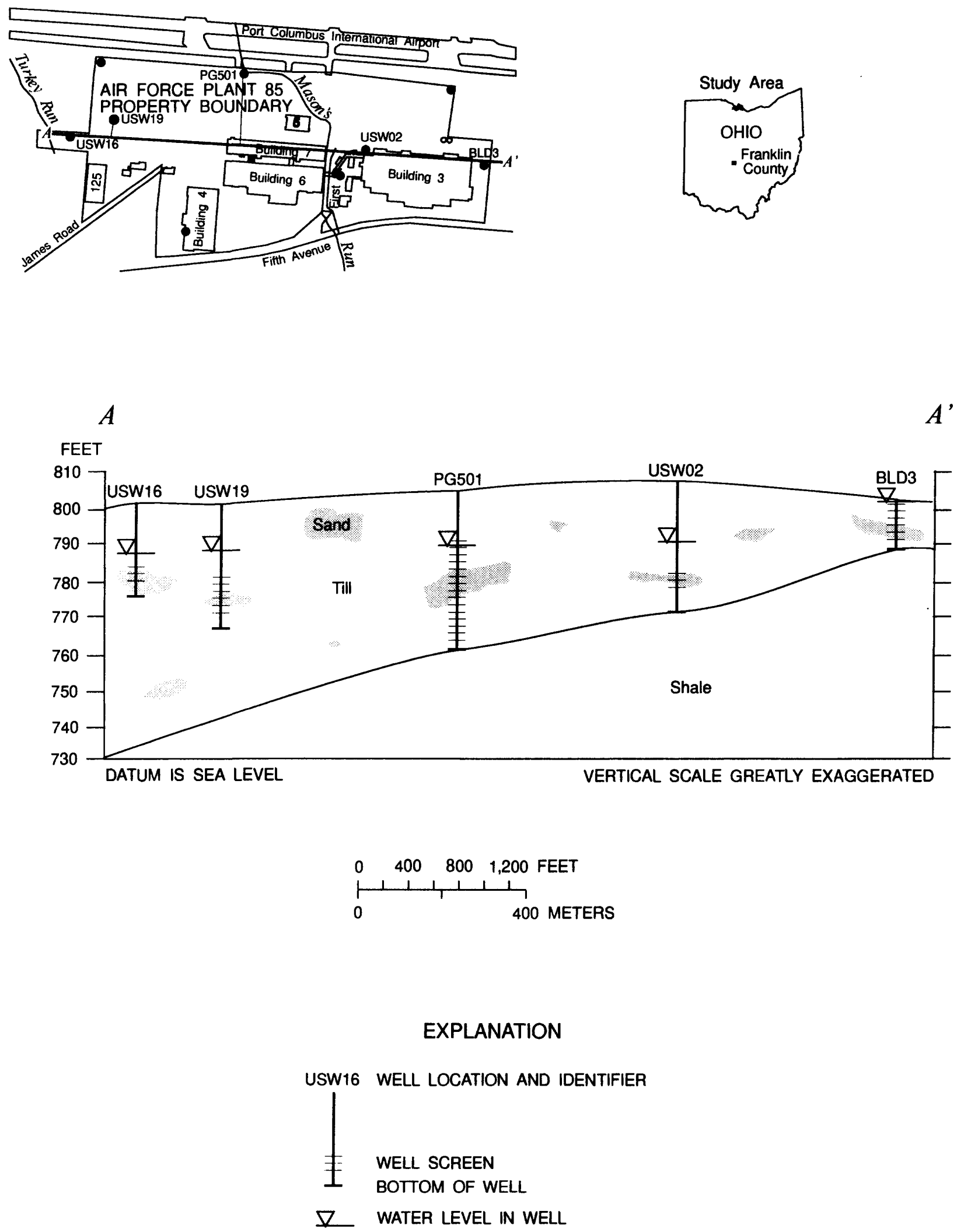

Figure 13. Cross-section of $A-A^{\prime}$ showing monitoring wells at Air Force Plant 85 , Columbus, Ohio. 